\title{
Limnephilid taxa revised by speciation traits: Rhadicoleptus, Isogamus, Melampophylax genera, Chaetopteryx rugulosa, Psilopteryx psorosa species groups, Drusus bolivari, Annitella kosciuszkii species complexes (Trichoptera: Limnephilidae)*
}

\author{
J. OlÁH ${ }^{1}$, P. ChVOJKA ${ }^{2}$, G. COPPA ${ }^{3}$, R. J. GODUNKO ${ }^{4,5}$, O. LODOVICI ${ }^{6}$, K. MAJECKA $^{7}$, J. MAJECKI $^{7}$, \\ B. SZCZĘSNY ${ }^{8}$, G. URBANIC ${ }^{9,10} \&$ M. VALLE ${ }^{11}$
}

\author{
${ }^{1} J a ́ n o s$ Oláh, residence postal address: Tarján u. 28, H-4032 Debrecen, Hungary.E-mail: profolah@gmail.com \\ ${ }^{2}$ Pavel Chvojka, National Museum, Natural History Museum, Department of Entomology, Cirkusová 1740, CZ-193 00, \\ Praha 9, Czech Republic.E-mail: pavel_chvojka@nm.cz \\ ${ }^{3}$ Gennaro Coppa, 1, rue du Courlis, F-08350 Villers-sur-Bar, France. E-mail: gennaro.coppa@wanadoo.fr \\ ${ }^{4}$ Roman J. Godunko, State Museum of Natural History, National Academy of Sciences of Ukraine, Teatralna 18, \\ 79008 Lviv, Ukraine \\ ${ }^{5}$ Biology Centre, Czech Academy of Sciences, Institute of Entomology, Branišovská 31, 37005 České Budějovice, Czech \\ Republicgodunko@seznam.cz \\ ${ }^{6}$ Omar Lodovici, Museo Scienze Naturali “E. Caffi”, Piazza Cittadella 10, I-24129 Bergamo, Italy. \\ E-mail: olodovici@comune.bg.it \\ ${ }^{7}$ Katarzyna Majecka and Janusz Majecki, Department of Experimental Zoology and Evolutionary Biology, University of \\ Lódz, Banacha 12/16,90-237Lódz, Poland.E-mails: kmajecka@biol.uni.lodz.pl,jmajecki@biol.uni.lodz.pl \\ ${ }^{8}$ Bronislaw Szczęsny, Institute of Nature Conservation, Polish Academy of Sciences, Kraków, Poland. \\ E-mail: szczesny@iop.krakow.pl \\ ${ }^{9}$ Gorazd Urbanic, Institute for Water of the Republic of Slovenia, Hajdrihova 28c, 1000 Ljubljana, Slovenia \\ Email: Gorazd.Urbanic@izvrs.si \\ ${ }^{10}$ University of Ljubljana, Biotechnical Faculty, Department of Biology, Večna pot 111, 1000 Ljubljana, Slovenia \\ E-mail.Gorazd.Urbanic@bf.uni-lj.si \\ ${ }^{11}$ Marco Valle, Museo Scienze Naturali "E. Caffi”, Piazza Cittadella 10, I-24129 Bergamo, Italy. \\ E-mail:mvalle@comune.bg.it
}

\begin{abstract}
Speciation traits of paramere, paraproct and aedeagus were applied to find initial split criteria with fine structure analysis in order to prepare diverged trait matrices for delimiting phylogenetic incipient species of unsettled limnephilid taxa in the early stages of reproductive isolation. A brief history is presented how this phenotypic taxonomic tool of the speciation traits was discovered and applied in caddisfly taxonomy. The theoretical basis was elaborated for the phenotypic speciation trait by reviewing several relevant topics in the sciences of taxonomy, molecular genetics and phylogenetics. Perspectives of integrative taxonomy is discussed in context of phenotype versus genotype, immensely complex phenotype versus phenomic challenge, taxonomic impediment versus genetic expedient, taxonomic adaptation of genetic vocabulary versus genetic sophistication and virtualization, New Systematics of Huxley and Mayr versus New Taxonomy of Wheeler. Debates on magic trait, speciation phenotype, speciation trait and super traits are discussed concluding that evolution works with phenotype and why the cryptic species concept is irrelevant. Briefly summarized how speciation traits evolve in sexual selection, through accelerated reproductive isolation with genital evolution through sex-limited speciation traits, including minor sex chromosomes. Why neutral molecular markers are blind compared to the adaptive speciation traits sensitized by fine structure analysis and backed by the potential of high-tech and high-throughput phenotyping and cyber-infrastructure broadly accessible and fed by computable phenotype descriptions. What sort of genetics could really help taxonomy to describe biodiversity of the over 100 million unknown taxa? Collecting new and re-examining old type materials deposited in various collections, the following taxonomic actions were elaborated by speciation traits. Drusus bolivari new species complex has been erected with redescription of Drusus bolivari (McLachlan, 1876), with species status resurrection of $D$. estrellensis (McLachlan, 1884) stat. restit., with description of five new species: D. carmenae Oláh, sp. nov., D. gonzalezi Oláh, sp. nov., D. grafi Oláh, sp. nov., D. gredosensis Oláh, sp. nov., D. jesusi Oláh, sp. nov., D. pyrenensis Oláh \& Coppa, sp. nov. Genus Isogamus is revised with description of two new species: I. baloghi Oláh, sp. nov., I. balinti Oláh, sp. nov. Melampophylax genus revised with one new species cluster: M. nepos, with two new species descriptions: M. keses Coppa \&
\end{abstract}

urn:lsid:zoobank.org:pub:DD21C9A2-2104-4EE6-8F49-ADBDE1AC81C6

HU ISSN 2063-1588 (online), HU ISSN 0237-5419 (print) http://dx.doi.org/10.18348/opzool.2015.1.3

*This article is dedicated to the memory of the late Prof. Dr. Sándor Mahunka, ordinary member of HAS. 
Oláh, sp. nov. M. szczesnyorum Oláh \& Chvojka, sp. nov., with three new species status: M. banaticus Botosaneanu, 1995 stat. nov., M. gutinicus Botosaneanu, 1995 stat. nov., M. triangulifera Botosaneanu, 1957 stat. nov. Rhadicoleptus genus revised with redescription of $R$. alpestris (Kolenati, 1848), with three new species status: $R$. macedonicus Botosaneanu \& Riedel, 1965 stat. nov. R. meridiocarpaticus Botosaneanu \& Riedel, 1965 stat. nov. R. sylvanocarpaticus Botosaneanu \& Riedel, 1965 stat. nov., with one species status resurrection: R. spinifer (McLachlan, 1875) stat. restit. Based on paramere evolution the Rhadicoleptus genus is transferred from the tribe Limnephilini to Stenophylacini. Annitella kosciuszkii new species complex has been erected and revised with redescription of A. chomiacensis (Dziędzielewicz, 1908), A. lateroproducta (Botosaneanu, 1952), with one species status resurrection: A. kosciuszkii Klapálek, 1907 stat. restit., with description of a new species: A. wolosatka Oláh \&Szczęsny, sp. nov., with two new synonyms: A. dziedzielewiczi Schmid, 1952 synonym of A. kosciuszkii. syn. nov., A. transylvanica Murgoci, 1957 synonym of A. kosciuszkii. syn. nov. Chaetopteryx rugulosa species group revised with description of five new species: C. balcanica Oláh, sp. nov., C. karima Oláh, sp. nov., C. kozarensis Oláh, sp. nov., C. psunjensis Oláh, sp. nov., C. tompa Oláh, sp. nov., with three species status resurrections: C. papukensis Oláh \& Szivák, 2012 stat. restit., C. prealpensis Oláh, 2012 stat. restit., C. zalaensis Oláh, 2012 stat. restit. Psilopteryx psorosa new species group erected and revised with three new sibling species complexes: $P$. bohemosaxonica, $P$. carpathica, $P$. psorosa, with two new species descriptions: $P$. javorensis Oláh, sp. nov., $P$. harmas Oláh \& Chvojka, sp. nov. with one species status resurrection: P. carpathica Schmid, 1952 stat. restit., with three new species status: P. bohemosaxonica Mey \& Botosaneanu, 1985 stat. nov., P. retezatica Botosaneanu \& Schneider, 1978 stat. nov., $P$. transylvanica Mey \& Botosaneanu, 1985 stat. nov.

Keywords. Speciation traits, neutral and adaptive molecular markers, neutral and adaptive traits, cryptic species, sibling species, incipient species, sexual selection, genital evolution, phenomics versus genomics, new species.

\section{INTRODUCTION}

$\mathrm{T}$ he central role of the parameres in the early stages of speciation processes of certain caddisfly taxa, driven by sexual selection, was recognised and information slowly accumulated in our previous studies on limnephilids (Oláh et al. 2012; Oláh et al. 2013a, b, c; Oláh et al. 2014). Paramere and aedeagus diversity, under the term of morphological polymorphism, has already been recognised earlier in the Potamophylax cingulatus species complex (Szczęsny 1990). In this paper we give a historical overview how this phenotypic taxonomic tool was discovered. We review its theoretical background and apply it to revise further unsettled limnephilid taxa, all having closely related incipient species: Rhadicoleptus, Isogamus, Melampophylax genera, Chaetopteryx rugulosa, Psilopteryx psorosa species groups, Drusus bolivari, Annitella kosciuszkii species complexes.

Our reliance on fine structure analysis of reproductive traits is based on empirical diversity evidences evolved by sexual selection. Therefore we instigate taxonomists for more sophistication in the phenomics of alpha taxonomy. We discuss several aspects of how parameres or additional speciation traits participate in speciation and how we can use these traits to delimit incipient species. Why these phenotypes are more sensitive and applicable in detecting reproductive isolation than the genotypes of neutral DNA markers? Why phenomics needs to get importance over genomics in biodiversity research that is in taxonomy? Why has the new taxonomy shifted the primary emphasis in taxonomy from genomic basis to diverging phenotypes of incipient species? Why speciation phenotypes have new evolutionary perspectives in sexual selection? Trials to answer all these questions, taxonomists need to learn more how to adapt in taxonomy the relevant part of the knowledge, principles, theories, models, algorithms, and procedures accumulated in genetics.

For instance, why don't we use Pst, an approximated outlier analogue of Qst or Fst, to compute the phenotypic divergence of parameres, in case if not visible properly with the microscoped eye of the taxonomist? Why taxonomist and geneticist do not team for simple Pst - Fst or for complex Qst - Fst comparisions? Both would greatly contribute to distinguish between natural selection and genetic drift as causes of divergence of speciation traits instead of giving jobs for technicians or students to measure more neutral markers or barcoding (Leinonen et al. 2013). In return, empirical phenotypes may contribute to resolve the desperate dominance of theories and models either in genotyping or in genome typing. This empiricism of reality would perhaps alleviate the excessive virtuality of molecular genetics. 
The divergence of the parameres was rather indicative and convincing in our previous studies conducted on the Chaetopteryx, Chaetopteroides, Oxyethira, Potamophylax and Allogamus genera. The most striking fact what we have experienced again, when started to examine the targeted new unsettled taxa, one by one, that all taxa have evolved consistently shaped parameres. Paramere divergence, even if measured delicate, proved to be very stable both between and inside populations. We realised that diverged parameres, just alone, could be effectively used to distinguish and delimit incipient species. If paramere divergence was not consistent enough we have searched auxiliary male and female fine structures, presumably involved more directly in copulatory processes. Depending on particular taxa, we have found aedeagus, paraproct, gonopod, and vaginal sclerite complexes participating directly in the early stages of speciation processes.

\section{DISCOVERY OF SPECIATION TRAITS}

The phylogenetic species concept in sexual selection processes was applied to separate and describe seven new species in the Chaetopteryx rugulosa species group by detecting fine, but stable and consistent morphological divergences both on the paramere and on the aedeagus of the phallic organ (Oláh et al. 2012). However, the rest of the genitalia were very unstable in the examined 192 populations. Significant intrapopulation variability has been experienced in the shape of all periphallic organs: cerci, paraproct, and gonopods. High rate of combined influences in various ration of (1) the effective population size sensitive genetic drift, (2) of the migration dependent gene flow and (3) of the environment triggered phenotypic plasticity could have been resulted in this significant periphallic variability. In traditional caddisfly taxonomy the species delimitation is based primarily on the divergent shapes of these periphallic non-intromittent genital structures. Parameres were only seldom examined. Procedure to delimit taxa with cerci, paraproct, and gonopods works effectively in well-separated old species. Their variation ranges are well inside the diverged ranges of the species. Difficulties arise when we are faced with closely related siblings of young, incipient species.
In a synopsis on the Oxyethira flavicornis species group (Oláh \& Ito 2013a) we have confirmed our finding on the diversity and stability of the intromittent organ recognised first in the C. rugulosa species group. The phallic structure, and especially the paramere in the $O$. flavicornis species group, was particularly diverse and stable. We have found the shape divergence of the complex parameres very effective in distinguishing the closely related species among these tiny creatures.

The small Chaetopteroides genus with combined chaetopterygini and stenophylacini characters was revised with description of three new species (Oláh et al. 2013b). Again the paramere, the copulatory courtship device with possible multiple titillating, harming, cleaning, anchoring, and several other additional functions, was the most diverse and stable character to separate siblings in this young genus. A sequence of diversity rate has been established from non-genital structures through non-intromittent genital structures to intromittent genital structures. We have distinguished two diversity parameters subject to the speciation processes of the parameres in mate preferences: (1) the strength or rate of preferences determines how rapid is the diverging speciation process and (2) the amount of divergence in the preferred trait determines the magnitude of diversification, that determines how easily we are able to recognise shape diversification in this early steps of speciation.

How to recognise early stages of divergence? Our experiences with the Chaetopteryx, Chaetopteroides and Oxyethira phallic organs, and the need recognising the phylogenetic species in the obscured Potamophylax nigricornis taxon inspired us to initiate detailed examination of the fine structures on the phallic organ and in the vaginal sclerite complex directly involved in mating (Oláh et al. 2013c). We have adapted the principles of fine structure analysis with pinpoint precision in recognising the split of phylogenetic species along a continuum of divergence in order to find stable fine structures nearby the structural diverging point. We have found that the $P$. nigricornis, a spring dwelling caddis fly species widely distributed in Europe does not belong to a 
single species. It forms an entire species group with seventeen peripatric incipient species. The ancestral complex paramere of $P$. nigricornis with sophisticated setal pattern was extraordinary stable in the examined 106 populations. Evolution of the phallic structure was examined by recording simplifications in paramere and modifications on the head of the aedeagus. Phenotypic plasticity increases, the interrelated processes of canalization, developmental stability, and morphological integration decrease and control the intraspecific variation. Due to this intraspecific variability there is an important premise when the fine structure analysis is applied to find stable trait for initial split criteria: we need to examine several specimens in several populations.

Darwin's innovation, the population thinking was realised when we introduced the principle and procedure of the empirical "diverged structure matrix" for the examination of structural diversification nearby the diverging point (Oláh et al. 2014). We search the first morphological product of speciation, presumably an adaptive, non-neutral trait, examine many specimens form many populations and produce simple diagrammatic drawings of the just diverged structure of all the examined specimens. If divergence is very distinct we draw and publish only a representative number of specimens. Drawings are arranged in a matrix network to provoke a concentrated visual effect for mental experience and to symbolize that this network is the "mother", the maternal material, or matrix of the just born species. If somebody examines several specimens, one by one under microscope during several hours, got an impression, enjoy details of variability and finally see the forest for the tree or getting frustrated and misled by individual alterations. We already surprised how much admire and convincing is to experience population reality very effectively when demonstrated together in a matrix. Not individuals and not abstract equations! This published matrix of diagrammatic drawings may serve as maternal material for further detailed shape analysis, if required, applying the new developments of geometric morphometrics (Adams et al. 2013). If variation range overlaps traditional taxonomic practice of visual species delimitation, geometric morphometrics may help to determine species boundaries. This matrix presentation of the diverged delicate trait generated by fine structure analysis is generally satisfactory to separate closely related incipient species in the practice of alpha taxonomy. Comparing the visual empiricism of morphological phenotype research to the desperate theoretical and model thinking of genotype research we have to acknowledge if visual delimitation of closely related species is available and applicable to separate incipient species without the fabricated speculative molecular genetic models (Oláh et al. 2014).

To evaluate the evolving direction of divergences from the discovered delicate structures, that is to estimate whether the complex is the plesiomorphic and simple is the apomorphic, or the opposite, we have reviewed contradicting debates emerged in recent theoretical studies. The old Williston's law of evolution towards reduction has been confirmed. It was concluded that reduction in the number of structural parts could be associated with increasing complexity composed by complementary qualities. This conclusion was perfectly supported by the pattern of paramere fusion inside the large and divergent Allogamus genus. The apparently more complex is the ancestral plesiomorphic state of the parameres and the simplified fused paramere with inbuilt high complementary complexity is the apomorphic state of the phallic organ. This pattern of paramere evolution guided us to establish species groups and subgroups inside the Allogamus genus (Oláh et al. 2014).

It was an instructive lesson to realise this extraordinarily high comparative rate of diversity on structures of the intromittent phallic organ. High rate of paramere divergence has been demonstrated in practically all of the examined, so called dubious, unsettled genera and species complexes listed and briefly summarized above. These taxa were unsettled because they are incipient species just diverged or diverging under sexual selection in allopatry, usually in isolated springs and spring streams on high mountain elevation. These finding definitely suggest some kind of runaway coevolution coupled and enforced by cryptic fe- 
male choice operating on an unknown genomic segment in the sexual selection processes. We have found suggestive and convincing the remarkable stability of these selection targeted structures as have been documented by diverged trait matrices.

High rate and stability of divergences confirms that these diverged structures were generated selectively by non-neutral and non-random adaptive mechanisms. The neutral (non-selective) and stochastic mechanisms of mutation, genetic drift and gene flow operate on the entire genome and on random pattern. Even the combination of the nonneutral and neutral evolutionary mechanisms by selective sweep, reduced recombination, or genetic hitchhiking processes, that is the effect of local or sexual selection on linked neutral selection is expected to extend over only a very small chromosomal region (Bierne et al. 2011). Based on these finding we presume that these delicate structures directly involved in copulatory processes are speciation traits evolving under sexual selection with various reinforcement mechanisms in local adaptation. We have realised that these speciation traits of morphological phenotype are unique devices applicable easily and very effectively in alpha taxonomy. If such an adaptive speciation trait under selection is recognised, a simple microscope is sufficient to distinguish and delimit the diverged or even diverging incipient phylogenetic species.

One premise is however important to meet. If the diverged trait is delicate, looks tiny for human eye or negligible for unsophisticated mental approach, one have to apply the population thinking and examine more specimens in more populations in order to produce diverged trait matrices. It seems that these taxonomic traits of phenotype are more informative than genetic tools of barcoding or concatenating neutral markers. Taxonomists have to learn achievements, limits, and shortcomings of molecular genetics in order to understand why speciation trait is more applicable in taxonomy, or why phenotype is more prospective. What is the prospect of phenomics versus genomics in alpha taxonomy? What is the speciation trait? Does the speciation phenotype diverge under directional sexual selection? Why neutral markers are blind for alpha taxonomy? What sort of molecular genetics could really help our practice in alpha taxonomy to fulfil our duty to describe biodiversity?

\section{THEORETICAL BASIS}

Taxonomy, the most tedious of all biological endeavours, is the oldest profession and engaged in detecting, observing, collecting, identifying, measuring, describing, illustrating, and classifying living creatures. Taxonomy has integrated the entire science of natural history for centuries. Taxonomy was fundamental to all other biological disciplines, and is the necessary foundation of any biodiversity research. Taxonomy is an integrative science from the very beginning in the sense as taxonomists collected and integrated all the observations or any available life history information for their groups during many years of field, laboratory, and library studies.

Integrative taxonomy. This old practice has got emphasis recently under the term of integrative taxonomy as an alternative against, and to alleviate, the peril of barcoding (Will et al. 2005) or to improve failures of neutral markers or increase rigour to delineate species boundaries (Dayrat 2005). Multiple, complementary perspectives of phylogeography, comparative morphology, population genetics, ecology, development, and behaviour have been concatenated to integrate all available data sources to frame species limits. However, integration has limits of complexity difficult yet to resolve (Yeates et al. 2011): (1) species are lineages; (2) species boundaries and relationship between species are biologically linked, but methodologically uncoupled; (3) concatenation is restricted to molecular data; (4) software implementations address only molecular data; (5) studies are correlative or corroborative rather than integrative; (6) lack of repeatable and quantifiable integration; (7) working near the species boundary; (8) incomplete lineage sorting and gene flow modify the history of various genetic loci. Integrative taxonomy is a surrogate of suppressed and deprived taxonomy, at best it could be realized as an iterative taxonomy practiced to test and retest species boundary hypo- 
theses again and again with new data sources (Yeates et al. 2011). Even iterative taxonomy is extremely resource intensive. Therefore we can study the integrative species boundaries on a very limited number of model organisms. During this lesson we learn at least the collaborative, integrated, and electronic future of taxonomy (Johnson 2011).

Vocabulary and sophistication. The taxonomy sensu stricto with its ever improving phenomics has own potential to collect and describe the unknown $c a .100$ million taxa. During centuries the traditional taxonomy was a synthetic discipline and integrated all the biological knowledge of taxa. Time is here to transfer the usable fraction of the genetic knowledge into the daily practice of taxonomy. We need genomics, in such a perspective. The accumulated knowledge of genomics is manifested into elaborated principles, theories, models, and laws. This knowledge is abbreviated into scientific vocabulary or terminology seldom applied in phenomics. It is the time for taxonomist to apply the value of this vocabulary of the codes into the expressed decoded reality. Barcoding or concatenating neutral markers have usable information as police fingerprint to detect various phenomena including identity and life history processes. But genetics, as an independent particular discipline, may help taxonomy in a more sophisticated way, for instance by genotyping speciation traits, such as the parameres that we have found participating in early reproductive isolation. This would help significantly our studies on speciation phenotype to fulfil the core mission of taxonomists to discover and describe biodiversity in early divergences of incipient species. Unfortunately the New systematics of Huxley and Mayr has retarded taxonomy upon the morphologically well separated architecture of the "adult" biological species and not recognised and practiced the phylogenetic incipient species diverged or diverging by fine structures of the reproductive barriers.

Data filtering. Today most teams of taxonomists and geneticists remained on the old pathway of trying to couple phenotype of traditional gross morphology (not fine structure!) with rou- tine neutral markers. They are lucky if, by accident, gross shapes and molecules fit to each others if not Procrustes superimposition starts working. Masking industries developed to cloak and obfuscate inconvenient data by pruning rogue data. Various types of data-smoothing techniques have been elaborated, to manipulate discordant, ambiguous data of the reality. Filtering algorithm was developed and got widespread use to assess phylogenetic anomalies and to remove unruly insertions, deletions, insufficient phylogenetic signals and other type of phylogenetically discordant sequences (Tomkins \& Bergman 2013). In Procrustes's bed we face the phenotype versus genotype scenario to answer the question what the speciation trait is, and why it is important in the practice of taxonomy. This helps also to understand why geneticists and their neutral markers are blind in alpha taxonomy and what sort of molecular genetics could really help our practice indirectly through phylogenetics. Phenotypes are observable characters or traits of varying states above the molecular level.

Speciation traits of deprived taxonomy. This phenotypic "observable and measurable" reality, so rich and unexplored, became a neglected field by resource removal after the Modern Synthesis of genetics, palaeontology and systematics arrived to replace taxonomy. Most of the available funds were consumed by genetics in the name of taxonomy, under the pharisaic political correctness. This scientific disaster has greatly suppressed taxonomy in spite of the fact that the phenotype, and not the individual gene or genotype is the subject of natural and sexual selection. Long awaited good news that "New Taxonomy" of Wheeler is going to return the lost ideas, principles, and practices of the empirical reality. We have to move from prevailing narrow perspective based on genome of a handful of organism and start to explore the endless forms of developing species phenotypes in describing biodiversity (Butlin et al. 2012). The speciation traits of the phenotype, whether visual, acoustic, olfactory mating signals or copulatory fine structures are direct empirical characters, applicable without theory and model fabrication, as well as easier and cheaper to explore. These questions are 
especially relevant for our parameres, just recognised and getting more available to separate incipient caddisfly species in the early stages of their adaptive sexual selection processes. Speciation traits can be used as a traditional character in alpha taxonomy more effectively in order to balance the blind neutral molecular markers at least on the funding market of biodiversity. This judgment however needs a wider insight.

\section{Phenotype versus genotype}

Taxonomic impediments. The parameres like to any other trait, or the phenotype in general became much underestimated as a speciation phenotypic tool when the epoch of New Systematics arrived and started to dominate. The New Taxonomy of Wheeler (2008) is a return to the bold vision of Linnaeus, Darwin, Hennig, and a deliberate reversal of the ill effects of the New Systematics of Huxley (1940) and Mayr (1942). The conflation of systematics with genetics that is the "modern synthesis" led to the present controversy: "Contemporary taxonomy derives from a caustic political correctness founded in ignorance of the epistemic basis of taxonomy, and in unwarranted arrogance regarding experimentalism". "Time has come for taxonomist to pull together and demand the support that they need for research, education, collections, and cyberinfrastructure". "We are the last generation with the opportunity to fully explore the diversity of the life on this little-known planet" (Wheeler 2008). The core mission of taxonomy is to collect, discover, describe, and classify units of biodiversity. The discipline of phylogenetics is an important adventure to understand relationships based on an old idea predated Darwin, that species are related through a history of common descent and organised through permanent diverging power. This idea supplies us with principles and procedures how to understand the diversifying mechanisms in speciation processes that produce biodiversity and how to manage diverging taxa greatly endangered by extinction.

Phylogenetics is not a science to discover and describe taxa. Taxonomy has proper techniques but lacks adequate funding to accomplish this task. High-tech and high-throughput procedures are available, but low financing prevents automatization and education of highly trained personnel. Taxonomy has been marginalized and underfunded in the century of biodiversity extinction. Does the mission of taxonomy survive the liberal dictates of the "Modern Economic Man"? Are we able to survey the unknown biodiversity? So far we have succeeded to describe less than 2 million species out of some 100 or even more million. We are unable to keep the race of describing over killing the biodiversity. The description of specimens in the century of extinctions is much more urgent than clarifying their phylogenetic relationships. Evolution seems not teleological: all the information encoded in biodiversity will be lost forever (Dubois 2010).

Lack of taxonomists to effectively describe the remaining biodiversity on earth is a real taxonomic impediment. There is another taxonomic impediment that is an "expedient": the diverse human character. Few taxonomists enjoy the entire process in the publishing arena (Evenhuis 2007). Individual taxonomists have their habits and we have to rely on their own inherent activity pattern. Few of us complete all the eight steps leading to the taxonomic nirvana of species description. Both the limiting number and the resource-limited activity of taxonomist contribute to the present scenario. Next taxonomic impediment is the national laws emerging worldwide to limit the collection of specimens. These regulations are legitimized to protect natural populations and species, but impeding professionals to carry out taxonomic research. But how to protect what is still unknown (Dubois 2010). The worst taxonomic impediment is that resources are usurped by those who need the products of taxonomic research (including ecologists, population geneticists, and conservationists). Turning biodiversity research to the false direction of "molecular systematics" was accelerated by pharisaic financing policies of "funding would be impossible otherwise" (Flowers 2007). Real advances of molecular based phylogenies have come at a painful cost: a total devastation in taxonomy. Taxonomy, the real science of biodiversity has lost funding, job, and publication (Crisci 2006). 
Irrelevant cryptic species concept. Species are cryptic because taxonomy is deprived, phenotypic resolution is insufficient, and knowledge is limited. The so called highly variable widespread species are frequently composed of populations with large amount of undetected phenotypic divergences. Their morphology or phenotype is only superficially indistinguishable. Presumed dietary generalist of Coleoptera, Lepidoptera and other herbivorous insect species proved to be cryptic complexes of dietary specialist species (Bickford et al. 2006). Molecular genetics has addressed their species limits and revealed higher than expected genetic diversity expressing as well as supporting reality and identity of phenotypic divergences of the so called "cryptic species". Most of these species are pseudo-cryptic due to the inadequate study of morphological features, the limited knowledge of ecology, the unknown behaviour like nonvisual sound, vibration, pheromones, and electric mating signals or they are under strong stabilizing selection that promotes morphological stasis. They are cryptic simply because we are blind we apply inadequate phenotypic resolution. Similarly sibling or sister species are pairs or groups of closely related species near the invisible end of the spectrum of morphological species differences (Mayr 1953). Sibling and cryptic species are not separate taxonomic rank or category. They differ by minute structural or phenotypic differences. Mayr (1942) has coined the term sibling species for such pairs or groups of morphologically nearly identical species. The previously existing German term Geschwister-Arten (sibling, Ramme 1930) and French term especies jumelles (twin, Cuénot 1936) were compromised to English. Sibling species are called also as biological or physiological species, cryptic species or phenons (Mayr 1953). The existence of this false sibling and cryptic species concept formed the central idea of Mayr's attack on the morphological species concept contributing to the early triumph of molecular phylogenetics.

Most of the sibling and cryptic incipient species have not been subjected to taxonomic revision using advanced methods. Usurped funding from taxonomy prevented to renew the 50-200 years old monographs and revisions. The pace of progress towards completing the encyclopaedia of life (Wilson 2003) is too slow. At the present rate this ambitious project will reach completion in 2800 years (Scotland et al. 2003). Moreover the aim of an encyclopaedia of life is to make species descriptions and taxa revisions freely available online; however copyright law prevents this and the main obstacle to developing a global taxonomy network is the lack of taxonomists themselves (Agosti 2003). The available old revisions are produced without modern techniques of high resolution and clearing procedures and remained full of lumped species even among the most studied European taxa. Under a single name of the European spring dweller caddisfly species, Potamophylax nigricornis we have found seventeen new peripatric species by simple fine structure analysis (Oláh et al. 2013c). Here we describe eight incipient species determined earlier as a single species, Drusus bolivari inhabiting the Iberian mountain ranges.

Infinite potential of fine structure analysis or visual and acoustic courtship signal as well as other complex ecological, behavioural, and physiological phenotypic procedures backed by cyber-infrastructures are now available to recognise early stages of divergence. We can search initial split criteria to detect diverging spots by fine structure analysis, and if required by geometric morphometrics for shape analysis or by applying population principles of many specimens from many populations, if necessary to determine trait stability. No doubt that discovery of putatively new species based on DNA sequences also enlarges our opportunities in taxonomy, but combined studies of integrative taxonomy concentrating on the more diverse and complex phenoltypes is the new taxonomy (Wheeler 2008).

Immensely complex phenotype. A recent cytochrome $b$ divergence synthesis on 600 avian sister species pairs has computed negative correlation between cyt $b$ divergence versus generation length and body mass (Thomson et al. 2014). Sad to realise that this old phenotypic evidence is the only final conclusion of the extensive and intensive molecular studies conducted on incipi- 
ent avian species. We have learnt from many other molecular studies that the minimal accumulation of indirect divergence in this neutral marker is rather blind and not sensitive enough to detect the real on-going adaptive selection processes, those creating the divergences on relevant loci in the early stages of speciation. To map the evolving genomic hot-spot (gene-finding) is remained mostly as one of the promises of molecular genetics at least on taxonomic scale, perhaps a hope of future. With fine structure analysis we are able to target directly the speciation trait easily and cheaply. With its present knowledge the molecular genetics is unable to do the same on taxonomic scale. Taxonomy is still eager to receive the promised and awaited genomic background of the diverging phenotypic traits such as parameres of the described species. It seems however that this was an early naive wishful desire dreamed by taxonomy and propagated by molecular genetics that is by the funding beneficiary. Most phenotypes are very complex and expressed by multigenic genomic processes, including pleiotropy and epistasis, through complex regulatory mechanisms and epigenetic interactions. Thousands of polymorphisms are implicated to explain the observed genetic variation. There are quantitative genetic models which operate with effectively infinite number of variation. There are phenotypes expressed by thousands of genes and millions of variants. Moreover the classical genotype phenotype distinction is slowly modifying into a single Holon. The code and coded is getting manifested into a complex reality of interwoven coding (environment)-encoding (phenom)-decoding (genome) universe of environmentally mediated interactions. The information contents of phenome dwarves those of genome (Deans et al. 2015).

Phenomic challenge. Recent flood of genomewide association studies has validated the use of phenotype-centred approaches to search inheritance, instead of producing more and accumulating all the genetic variants that influence the phenotype. The next challenge is phenomics: (1) acquisition of high-dimensional phenotypic data on an organismal wide scale; (2) genotypephenotype mapping, the gene finding including Mendelian randomization experiments and many more sophisticated approaches designed to trace causal links between genotype and phenotype (Houle et al. 2010). (3) microsatellite-based linkage analysis by reciprocal outbreed crosses adapted to search relations between quantitative trait loci and shape variation of intromittent organ like aedeagus (Schafer et al. 2011) or paramere. How we find our way through this vast landscape of phenotypic data? Real progress in evolution and medicine research depends on genotypephenotype mapping. The causation of selection processes takes place in a continuous phenotype space without real knowledge of the relationship to the genotype space. Genotype study with minimal reference to phenotype is clearly insufficient to elucidate these phenomena (Houle 2010). Prospective studies suggest that traditional phenotypic risk factors, like obesity for diabetes, are more effective predictors for many diseases than single nucleotide polymorphism associations (Houle et al. 2010). Similarly how genetics, ecology and conservation are staggering in agony without sound taxonomy of phenomics. See the failed genomic war on cancer phenome (Spector 2010, Davies \& Lineweaver 2011, Davies 2013, Lineweaver et al. 2014).

Cope with genome. Phenotypes such as morphology, ecology and behaviour drive most research in life sciences. It is the time to get more prepared and cope with the genotype of the much financed molecular genetics. Combining information from genomes, transcriptomes, proteomes, phenomes, and biomes is backed by emerging informatics standards (Deans et al. 2012b). Hightech and high-throughput phenotyping is getting more and more available and employed in several fields of phenomics (Sozzani \& Benfey 2011). The vast and diverse landscape of phenotype data need to be processed by cyber-infrastructure broadly accessible and fed by computable phenoltype descriptions based on ontology terms and entity-quality formalism; by semantically represented phenotype data; by sets of processing algorithms combining logics of ontologies with statistics (Deans et al. 2015). It is the time to change how we describe biodiversity to ensure phenotype computable and linkable to digital data with semantic, extensible, and broadly accessible 
contents (Deans et al. 2012a). Breakthroughs in morphology of arthropod systematics, the promise of a renaissance of morphology is admired but limited to a handful of laboratories (Popper \& Schaffner 1959, Stuessy et al. 2003, Bud \& Olsson 2007, Friedrich \& Beutel 2008). Template principle for more effective species descriptions (Evenhuis 2007) and uniformed spatial descriptors for phenotypic diversity in the biological spatial ontology (Dahdul et al. 2014) are waiting to be applied. Methodological inertia, relying mostly on sclerites, limits the wide application of fine structure analysis including female structures and internal soft body parts full of important unexplored phenotypic traits (Deans et al. 2012b).

\section{Is paramere a speciation super trait?}

It is a great moment for taxonomy to find phenotypic trait simple and effective enough to distinguish among difficult, closely related taxa, and to establish species boundaries in highly varying species complexes. We have discovered such a trait in the very centre of the diverging speciation process creating reproductive isolation by sexual selection. If paramere trait fulfils the initial split criterion and permits to detect initial separation in the early divergence of an ancestral lineage we are authorized to qualify this trait as "super". For such a super trait there is an "hourglass neck" metaphor that theoreties how to find causation in the immensely complex and varying phenom.

The neck of hourglass. Buchanan et al. (2006) has introduced the metaphor of hourglass phenomenon to visualize our hope, a tiny chance to understand proximate causation of natural selection in the infinite Holon complexity of the genotype, environment, and phenotype triad. The complex genotype and the immensely complex phenotype, the many-to-many causal fabric, are represented by the two ends of the hourglass. The narrow hourglass neck, if we are fortunate enough to discover, may represent a factor maximally informative about the causation of natural selection. Any member of the triad, the environment, the phenotype, or the genotype could be a key factor (Houle 2010, Houle et al. 2010). This neck factor explains the phenomena that seem complex at other levels. We have found the paramere, as such a neck factor on the genotype-phenotype map, very indicative of divergence produced by sexual selection in the early stages of speciation and making causation relatively simple.

Evolution works by phenotype. Parameres, the phenotypic trait of limnephilids, the substructures of the intromittent phallic organ are directly involved in sexual selection. Reproductive isolation proved to be the most powerful way to conceptualize the species boundaries as popularized by the biological species concept (Mayr 1942) and approved by the phylogenetic species concept. There is a debate whether inability to interbreed is only an integrated signature effect, the by-product of selection, that is the accumulated loss of reproductive compatibility, or it is the primary cause of speciation, the creator, the mechanism of selection. Phenotypic divergences between incipient species suggest causal interactions between divergent trait and gene flow restrictions during speciation (Shaw \& Mullen 2011). Evolution works by screening phenotypes rather than genotypes. Epigenetics presents a growing diversity of mechanisms allowing inheritance of acquired phenotypes. A pluralistic model of heredity is now emerging (Bonduriansky 2012). Non-genetic inheritance by genetic encoding, biased mutation, developmental phenotype plasticity, phenotypic and genetic accommodation, organic selection, orthoplasy and genetic assimilation is recognised as giving more and more importance to phenoltypes (Oláh et al. 2013c).

Speciation phenotype or speciation trait. An integrative new taxonomy based on phenomgenom Holon applies, if required, all axes of differentiation to test alternative causal hypotheses of speciation. Which diverging phenotypes involved, redundant or neutral in suppressing gene flow? Speciation phenotypes are those traits whose divergence contributes to a reduction of gene flow (Shaw \& Mullen 2011). Phenotypic divergence, as a proximate instrument of speciation in sympatry or allopatry or via founder effect or reinforcement, rarely studied systematically. Speciation traits are expressed phenotypes of speciation genes whose divergence made a significant contribution to the evolution of reproductive isolation. These traits/genes are under- 
lying the process of speciation. Speciation genes contributing to reproductive isolation is difficult to distinguish, especially if the multiple genes participate early in an ongoing speciation process and concerted in quantitative trait loci. Why do we not search and use the expressed speciation trait, the speciation phenotype before sequencing runs too far ahead of morphological or functional analysis (Butlin et al. 2012).

Magic traits versus super traits. A trait is magic if having double function completes ecological divergence of natural selection by reproductive isolation. A trait is magic because simultaneously involved in the processes of ecological divergence and in the evolution of assortative mating. It is a very efficient driver of speciation in sympatry with significant gene flow because the two functions will be not disassociated by recombination. Gene flow and recombination may randomize gene associations between divergent selection and non-random mating established in the processes of divergence hitchhiking and accumulated in linkage disequilibrium. This chance for disassociation disappears or alleviated by a magic trait if non-random mating is based on a trait under divergent non-sexual selection expressed pleiotropically by the same genes. Magic traits are encoded by magic genes under divergent ecological selection and pleiotropically affecting reproductive isolation.

Magic traits are not rare (Servedio et al. 2011). Automatic magic traits are rather common and widely distributed. For instance, divergent selection on phenological or host traits leads to assortative mating via temporal or spatial isolation.

A trait is super if initiates and realise a speciation process mostly in allopatry by sexual selection processes. It seems that speciation trait is even more distributed, at least among limnephilid taxa evolved in allopatry of the high mountain crenon environment. Speciation trait encoded by speciation genes under divergent sexual selection creates reproductive isolation without any abiotic or additional social selection. Speciation trait manifesting reproductive isolation by powerful sexual selection does not need any other additional selection pressures. This is why speciation paramere is really super, not simply magic.
Speciation genes and speciation traits. In a broad definition speciation genes restrict gene flow between incipient species contributing to the splitting of two lineages. In a constrained definition speciation genes are genes contributing to an increase in reproductive isolation (Nosil \& Schluter 2011). Distinguishing such genes acting early in an ongoing speciation process is very difficult (Butlin et al. 2012). Reproductive isolation was considered and quantified mostly through reduced hybrid fitness by hybrid inviability and sterility, a rather late and restricted perspective. These genes may express many forms of reproductive isolating traits evolved in premating, postmating, prezygotic and extrinsic or intrinsic postzygotic sexual processes. Speciation genes predating postzygotic incompatibilities in the divergence process and underlying premating and prezygotic speciation traits, like the parameres in caddisflies are probably much more important and their study would be more prospective.

Important to quantify the effect size of the speciation gene, that is the magnitude of increment of a gene to the total reproductive isolation. Practically a current effect on a specific component is estimated only, but a gene may have effect on more than one component of the total reproductive isolation. Measuring, or at best estimating the speciation effect size of genes is required to distinguish the genes that mattered to speciation from those that did not. In theory it is decreasing with increasing number of genes. In practice the number of genes underlying somehow intrinsic post-mating isolation was as large as 191 in $D$. simulans and D. melanogaster and cumulative effects of multiple components can limit the effect size further as well as the earlier-fixing genes have little effect at the time of divergence (Nosil \& Schluter 2011).

Speciation genes affecting a component of reproductive isolation are usually very complex, represent entire gene networks, and their function are further complicated by transcript/translate regulatory and environmentally mediated epigenetic processes. Moreover morphological speciation traits, like parameres are coded by multitude of quantitative trait loci, frequently analysed by infinite gene models. This complexity may be also 
reflected in large inter-individual variation as well demonstrated in variable drug reaction responsible for more than 100,000 deaths each year in the US alone. Understanding gene background of any speciation genital traits is still a black box even in Drosophila. No time for triumphalism (Goldstein \& Weale 2001). Any speciation trait, the expressed reality of speciation gene complexes or gene islands do not flow readily between species, they may cease flow before flow cessation at neutral traits. Why do we not apply the empirical reality of the speciation trait, the decoded like parameres, if we are unable to locate their molecular codes? They are the expressed genes. They are very powerful in describing the unknown biodiversity, especially in the early stages of divergence.

\section{Paramere evolution by sexual selection}

The rapidly diverging paramere appears as a practical phenotypic tool to separate closely related incipient species. The examined limnephilid genera with limited dispersal ability evolved and still are evolving in isolated spring and spring-fed hypocrenons on the higher elevations of the Carpathian and Balkan mountains. These habitats are ecologically rather stable and uniform with similar thermal regime and food resource pattern. Founder effect and weak contact reinforcement may operate, but divergent or disruptive, directional sexual selection dominates probably the speciation events with weak or without any prior abiotic or additional social environmental pressures. It has been already demonstrated in millipedes, that sexual selection is the sole driving force to produce species richness that is many species via morphological diversification, when a lineage of organisms consists of highly divided populations owing to limited dispersal (Sota \& Tanabe 2010).

The paramere, this fine, but important subunit of the intromittent phallic organ in caddisflies is a very active and mobile tool surrounded by the periphallic organs of the primary genitalia. We say fine or delicate, just because these structures seem fine for us on human scale, especially if our mind and methods are not sophisticated properly.
At the same time they are very real and functioning, robust enough in the sexual selection processes of the caddis-world. It may perform various copulatory functions: courtship, harm, assessing, cleaning, anchor, guarding, secreted plug, living plug (Oláh et al. 2013b). Shape divergence of this active structure is a direct result of divergent sexual selection that is a common cause of early lineage divergence in animals and impacts gene flow through direct consequence to mating probabilities. Theoretically the divergence of paramere can be indirect through pleiotropy, epistasis or genetic hitchhiking of any traits or genes diverging under other selection mechanisms. Diverging traits are not always lead to speciation, but evolve intraspecific differentiation of polymorphism or accumulate differences in standing genetic variation without gene flow reduction.

Divergence by natural or sexual selection? Sexual selection is more effective than natural selection to generate new species (Butlin et al. 2012). To understand how speciation trait, as a reproductive barrier, evolves during accelerated speciation we have to summarize some relevant terms commonly used or misused in taxonomy. Artificial selection, admired also by Darwin, is a long practiced process by human cooperation. Natural selection introduced by Darwin (1859) as non-artificial, operating analogously to artificial selection without human intervention. Sexual selection introduced by Darwin (1871) as similar to, but distinct from, natural selection. Darwin has failed to introduce a complementary term to other forms of non-artificial selection. Moreover when he has developed his second evolutionary theory, the sexual selection, he posed a real challenge to his own theory of natural selection. Flegr (1996) has suggested using the term sexual selection as a special form of natural selection and introduced a new term "environmental selection" for all the other types of natural selection. However environment is strictly composed of all the abiotic and biotic influences and surroundings, including mates. Sexual selection is a form of social selection, similarly to various mechanisms of social cooperations and competitions (Lyon \& Montgomerie 2012). A possible alternative idea we prefer to consider is that ecological, sexual and social 
selection are all environmental mechanisms triggering various pressures in the natural selection. Moreover it is getting more evidence that natural cooperation among entities rather than selection organises our universe. Divergence in mating preferences may be a common first step in this process. Sexual selection, as a primary mechanism in the evolution of assortative non-random mating to accommodate reproductive isolation is a dangerous idea against the basic principles of the selectionist molecular genetics.

Ecological speciation is defined as the evolution of reproductive isolation through ecologically based divergent natural selection. Yes but finally, even in the present scenario, processes in the various pathways of the sexual selection mechanism create the reproductive traits, possibly triggered or canalised by natural selection. In a broad definition of sexual selection, traits that influence competition for mates are sexually selected, whereas those that directly influence fecundity or offspring survival are naturally selected. However cooperation brings all these together.

How effective is natural selection? Darwin's courtship devices evolve not through correlation with environmental factors. Secondary sexual traits can evolve without the input of natural selection. Darwin's really dangerous idea is that natural selection fails to explain the evolution of all forms and design (Prum 2012). Selectionist paradigm was also debated by the proposal of neutral theory (Kimura \& Crow 1964, Nei 2005), demonstrating that genetic variation is maintained mostly by neutral processes. MacArthurian (1972) paradigm, that competition and not cooperation structures the communities is also challenged by the involvement of stochastic processes (Hubbel 1979, Strong 1980). Today unified neutral theory of biodiversity and biogeography assume neutrality of individuals within communities (Rosindell et al. 2011). Patterns in species distribution do not depend on adaptive differences between species, but on random fluctuations, dispersal, and speciation (Kopp 2010). The study and valid demonstration of positive (adaptive) Darwinian selection in natural populations has remained still difficult and can be also difficult to verify whether genes that behave as outlier are genuinely adaptive (Liukart et al. 2003).

Divergence by sexual selection! The rapid divergence of reproductive genes is a general evolutionary pattern in animals. The investigated unsettled closely related species of limnephilid taxa differ mostly in genital structures directly involved in the internal insemination processes. In these taxa we have detected very consistent shape divergence in male paramere, aedeagus, and paraproct as well as in the female vaginal sclerite complex, without any ecologically relevant morphological traits. DNA sequence analysis also has revealed extraordinary divergence of sex-related genes, particularly between closely related taxa (Vacquier 1998, Wyckoff et al. 2000, Good et al. 2013). Political correctness has confiscated ecological causes as priority research target for potential mechanisms of sympatric speciation. However recent research has put more emphasis on the evolution of the mating structure with focuses on sexual selection alone (Doorn et al. 2004). Intraspecific sexual selection has been the sole driving force to evolve speciation trait that underlie reproductive isolation (Maan et al. 2004).

Environmental differences and/or allopatric isolation facilitate the evolution of reproductive isolation. Divergent sexual selection exhibits complex mechanisms in speciation and works or even dictates in concert with viability selection to achieve local adaptation. It may play a direct role alone without other environmental pressures to create reproductive isolation. Also it may have a central role throughout the speciation process, evolves early to initiate speciation and reinforcement favours sexual isolation later to avoid costly heterospecific mating. Experimental trials demonstrated that female discrimination between species and male traits was insensitive to habitat (Lackey \& Boughman 2014). High diversity in sexually selected traits inspired the hypothesis that divergent sexual selection can drive speciation. Natural selection and sexual selection can interact during speciation and various diversifying effects emerge from interactions between sexual selection and environmental heterogeneity (Maan 
\& Seehausen 2011). Most of the classical theoretical models and experimental studies are based on viability selection in spite of the fact that fertility rather than viability comprised the larger fraction of total fitness (Prout 1971). Hawaiian Drosophilidae has been long cited as evolved by explosive adaptive radiation, but sexual selection had played a greater role at least during the initial stages of species formation (Kaneshiro 2006).

Sexual selection mechanisms. All the examined limnephilid genera (Allogamus, Annitella, Chaetopteryx, Chaetopteroides, Drusus, Isogamus, Melampophylax, Potamophylax, Psilopteryx, Rhadicoleptus: Oláh et al. 2012, Oláh et al. 2013a, b, c, Oláh et al. 2014), have exhibited very pronounced sequences of diversity rate from nongenital structures through non-intromittent genital structures to intromittent genital structures of the phallic organ This mere empirical fact suggests a divergence mechanism driven by sexual selection. Sexual selection operates in relation to sensory manipulation in mating, to ability of sperm removal in sperm competition, to control fertilization in sexual conflict, and to induce post-copulatory sperm selection in cryptic female choice (Arnqvist 1997). All these mechanisms result in genitalia diversify much more rapidly than other body structures. Genitalia are more than twice as divergent in taxa in which females mate many times in polyandry (Arnqvist 1998). This comparative evidence has been revisited and confirmed by meta-analysis of Kraaijeveld et al. (2011). Detailed morphometric studies on genitalic traits compared to seven non-genital morphological variables in calopterygid damselflies have demonstrated strong directional postmating sexual selection in intraspecific evolution of the genitalic traits in allopatry (Rivera et al. 2004). The comparative rate of divergence observed in caddisfly taxa suggest that cryptic female choice, the postcopulatory equivalent of the classic Darwinian precopulatory female choice, is probably one of the most common mechanisms in the sexual selection.

However controversy has been accumulated and remained still unresolved; which mechanisms are actually effective in sexual selection. How sensory manipulation, sperm competition, fertilization control, sexual conflict, cryptic female choice and more complex phenotypic mechanisms dominate the processes in sexual selection. Is the sex really conflictual? Intense theoretical efforts, abstracted into multitude of virtual models as well as highly manipulative artificial experiments, very far from reality, have generated heated debate. Sexual conflicts, combined with parasitic selfish genetic elements of meiotic drive, transposable elements, biased gene converters, postsegregation drivers, cytoplasmic drivers, heritable organelles and microbes, form the complex fabric on the evolution of reproductive isolation. How species formation via genomic conflict constitutes the conflictual speciation (Crespi \& Nosil 2012). It seems that in these models, conflict rather then cooperation dominates the male and female interaction, but the net coevolutionary effect is certainly cooperative rather than antagonistic in accordance with the traditional sexual selection theory (Zeh \& Zeh 2003). Pluralistic and integrative approaches are formulating to understand nature's reality how the material organisation integrates even the conflicts by concerted cooperation. We are getting more and more convinced that cooperation organises, and not the selection evolves, the reality.

We need more comparative field observations and field experiments combined with structure, function, behaviour, and cline observation as well as less theoretical models and less laboratory clones. The functional role of these diverse genital structures is hidden inside the female during copulation and remains a neglected topic. The copulatory piece inserted into vaginal chamber may work as an anchor to secure coupling of genitalia (Sasabe et al. 2007). Very small morphological divergence may induce robust alterations in copulatory courtship behaviour patterns: waving, rubbing, licking, squeezing, kicking, tapping, jerking, rocking, biting, feeding, vibrating, singing, and shaking (Eberhard 2010). Small but consistent morphological divergence in paramere integration, disintegration, curvature, tenuity, tip pattern, surface pattern, and many more shape or surface modification, can deliver surprisingly great variety of courtship signals to the female. A small change in paramere structure encoded by complex genetic changes in quantitative trait loci 
manifested in alterations of the sclerite shape or in undetected muscle modifications may change the frequency and duration of the above listed 13 courtship behaviour pattern to a great extent, multiplying the internal courtship signal by several powers. Probably many surprising phenomena intimately related to genital diversity remain to be discovered by direct field observations, by studies on flash-frozen copulatory pairs or by mapping of muscle attachment and articulations.

Is reproductive isolation a signature effect or the initial cause of speciation? Is the reproductive isolation just a by-product of natural selection and the prime target of directional selection is another phenotype? Or could the reproductive isolation be a direct target of selection? Selectionist feel that emphasis on reproductive isolation obstructs the study of speciation as a product of divergent selection and shift the primary emphasis to environmental adaptation through natural selection (Shaw \& Mullen, 2011). There is emphasis (Bonduriansky 2011) that sexual selection, as an engine of ecological diversification, could carry populations through fitness valleys of maladaptive intermediate phenotypes between alternative niches on the fitness landscape.

Accelerated reproductive isolation. Rapid ecological speciation may occur on ecological time scale (contemporary evolution, Hendry et al. 2007). But how this fitness adaptation is confirmed by reproductive isolation? Adaptive divergence can produce reproductive isolation in dozens to hundreds of generations even if natural selection, female choice and male attractiveness each depends on different and very complex quantitative traits. Reproductive proteins of gamete generation, storage, usage, signal transduction, gamete recognition, and fertilization diverge rapidly, establishing barriers to fertilization (Swanson \& Vacquier 2002, Zhang et al. 2007). Incomplete reproductive barriers to gene exchange might accumulate in spatial isolation. Ecological adaptation with short timescale might couple with old pre-existing intrinsic incompatibilities providing an alternative interpretation of rapid speciation events (Abbott et al. 2013).

High ratio of non-synonymous to synonymous nucleotide substitution has been demonstrated in
Drosophila species for sex and reproductionrelated genes compared to catalytic, developmental and other genes (Civetta \& Singh 1998). Sex and reproduction-related genes are more diverged than genes without apparent reproductive function. This finding suggests that rapid directional sexual selection occurs during the early stages of speciation. However signature of directional selection was not evident between more distantly related species. Probably directional selection has been relaxed later under stabilizing selection. Among sibling incipient species, in the most examined model system of the Drosophila melanogaster complex, the shape of genital arch exhibited consistent differences and has been found rapidly evolving (Kulathinal $\&$ Singh 2004). Sexual selection has been demonstrated as an important mechanism in generating morphological innovations. Rapid and often adaptive evolution has been computed in the analysis of 2505 genes with reproductive functions across six species in the $D$. melanogaster complex with striking disparity in rates and patterns among male- and female-specific genes (Haerty et al. 2007). Male tissue-specific genes evolve faster than female tissue-specific genes. Proteins associated with the reproductive system are twice as diverse as those not related to reproduction. Rates of sequence evolution of sex and sex-related genes was dramatically higher compared to the 6004 other genes without sex and reproductive functions. Rapid evolution is now consistently associated with reproductive genes driven by female choice or by sexual conflict in the sexual arm races. Early studies, concentrated on genes of general cell metabolism (allozyme or mtDNA) having no direct relation to sex and reproduction-related genes, have been unable to detect rapid reproductive divergences. Next approach of mapping trials for candidate "speciation genes" with large effect without pool of genetic variation was also unproductive to recognise high rate of evolution of reproductive traits. A systematic genomic/proteomic approach was essential to discover rapid and adaptive evolution of sex and reproductive-related genes/traits (Singh \& Jagadeeshan 2012). Recent studies have identified very rapidly evolving species-specific new genes 
with important phenotypic effects on sexual reproduction (Chen et al. 2013). It is now clear that various new genes have key roles in rapidly evolving reproductive processes. All copies of a young species-specific gene in Drosophila melanogaster that underwent a strong selective sweep and expressed specifically in the testis, was deleted by chromosome engineering. The manipulated sperm without the new genes competed poorly against sperm from wild-type flies. (Yeh et al. 2012).

Genital evolution. Diversity, variability, and apparent rapid evolution of genital morphology are fascinating speciation events attracting increased interest and vivid focus of research. In caddisflies the sequence rate in morphological differentiation indicates highest divergence for intromittent organ and especially for the parameres. Recent developments both in phenomics (geometric morphometrics, fine structure analysis) and genomics (mapping between divergent genomic region and phenotypic traits) have the potential to facilitate linking of the strikingly rapid and divergent paramere evolution with the sexual selection.

The functional significance of individual components of the genitalia during copulation is still rather obscure. In the internally fertilizing animals, like caddisflies the postcopulatory sexual selection is regarded as an evolutionary engine to produce diversity and rapid divergence in the structural elements of the intromittent phallic organ. Various spiny structures are prominent element both of the aedeagus and paramere on the phallic organ of caddisflies. Comparative and correlation studies on diverged structures may help to understand these mechanisms. Highly artificial experimental studies have just started. Recently the competitive fertilization success of male's ejaculate, whose aedeagal spine length was manipulated by micro-scale laser surgery, was assessed by using high-resolution radioisotopic labelling. Male with longer species-specific sclerotized genital spines on aedeagus were more successful in gaining fertilization (Hotzy et al. 2012). The importance of male driven copulatory mechanics and strategies has been revealed in the rapid diversification of genitalia of Drosophila flies (Jagadeeshan \& Singh 2006). The low level of phenotypic variation in adaptive divergence of speciation traits observed in all the examined caddisfly genera may suggest a canalised development under stabilizing selection. Shape alterations are sometimes so tiny that morphological divergence could be detected only in quantitative curvature variations as measured by statistical analysis in the shape of aedeagus between two sibling Drosophila species (Franco et al. 2006). Inconsistency between mDNA and aedeagus morphological data in D. buzzatii species cluster may be the result of the higher rate of evolution of male genitalia compared to general metabolic evolution of mitochondria (Soto et al. 2007).

Evolution of female genitalia. Morphological and evolutionary research on genitalia is strongly male biased and this male bias worsened with time in spite of the fact that female genitalia can evolve rapidly and complex coevolutionary dynamics may shape both male and female genital structures (Ah-King et al. 2014). Function of the frequently much elaborated male armament is easier to understand if we examine counterbalancing structural evolution of female response against male wounding, like connective tissue enlarging in seed beetles (Ronn et al. 2007) or developing pocket-like structure in Drosophila (Kamimura 2007). We have elaborated and analysed the fine structure of the dorsal and ventral vaginal sclerite complexes in female caddisflies and found these internal genital structures diverse and species-specific (Oláh et al. 2013c, 2014, Oláh \& Kovács 2014). Frequently, various substructures evolved very high diversity in three dimensions, unfortunately too complex for drawing and for routine taxonomical studies. We have described size-coevolution of male aedeagus and female vaginal chamber in the species of Allogamus tomor Oláh (Oláh \& Kovács 2012) and A. zugor Oláh (Oláh \& Kovács 2014). Similar coevolution has been recorded between the male parameres and the modified duct of the accessory gland with lineage sorting in several species complexes in the Allogamus genus (Oláh et al. 2014). In Chaetopteryx schmidi species subgroup there is coevolution detected between the interlocking copulatory structures, the dorsal 
branch of male paraproct, and the female anal tube.

The internal soft structures of female reproductive structures accumulate significant morphological divergences and modern cleaning techniques and micro-computed tomography are available to detect gross or even fine structural divergences. In postcopulatory sexual selection of internally fertilizing animals it could be productive to measure the coevolution of the female internal reproductive traits. In seed beetles positive correlation have been found among primary reproductive organs that is between the ejaculate quantity of testis and the size of bursa copulatryx (ejaculate receiver), accessory glands (processing ejaculate and egg laying) and spermatheca (sperm storage) as well as female lifetime fecundity quantified by egg countings (Rönn et al. 2011). Premating male-male competition, postmating sperm competition and cryptic female choice may contribute to these positive correlations. Large protein-rich ejaculate delay female remating and adapting a defensive sperm competition as well as elevating mate fecundity.

Variability of genital structures. The parameres, or occasionally other speciation traits like aedeagus and paraproct, directly involved or intimately linked to copulation processes has rather stable and consistent shape as a result of directional sexual selection. At the same time we have found the periphallic organs of cerci, paraproct, and gonopods frequently highly varying inside local populations, especially in highly isolated habitats with low effective population size. This allelic difference in standing genetic variation, segregating within a population, may be attributed to selectively neutral changes of the genetic drift and may serve as depots of genetic variation. They may represent alternative forms nearly equally fit or acceptable, in term of survival and reproduction (Kulathinal \& Singh 2004). Greater genetic variation due to less selective constraints on genes increases evolutionary potential, influences epigenetic processes and helps to develop phenotypic diversity. Under such condition sexual selection may drive especially rapid divergence of sexual trait. Rapid fixation of alleles caused by selection or genetic drift of neutral alleles enhances reproductive isolation and accelerates also the production of incompatibilities in hybrids. However gene flow in isolated habitats and among less dispersive species is rather limited.

Genetic background of sex-limited speciation trait. Parameres as sex-limited trait, are expressed only in males, and encoded probably by sexlinked genes. Sexual dimorphism, from gonads through primary reproductive organs and genital structures to somatic differences in morphology, physiology, and behaviour, are frequently encoded by sex chromosomes or by sex-limited genomic regions on chromosomes $\mathrm{Y}$ and $\mathrm{W}$. According to the present concept the evolution of sexual dimorphism is facilitated by sex chromosomes, the only portion of genome that differs between males and females. However transcription profiling has indicated that thousands of genes across all portions of the genome contribute to sex-biased gene expression of both the gonads and the soma (Mank et al. 2008). We experience again that the reality is far more complicated than current theory allows, similarly to most of the theoretical speculations abstracted in models of molecular genetics (Mank 2009). Genes on sex chromosomes may initiate the cascade of developmental processes leading to sexual dimorphism, but later the responding genes need not to be on sex chromosomes, most are distributed on autosomes (Fairbairn \& Roff 2006).

Organisms with Z-W sex chromosomes, like caddisflies, butterflies, and birds often have more conspicuous male sexual traits than taxa with $\mathrm{X}-\mathrm{Y}$ sex determination, such as flies and mammals. Zlinked genes are especially important to species recognition. Runaway (sexy son) inheritance is more likely in Z-linked preference while good gene inheritance is particularly powerful under Xliked and autosomal preference. Male-driven evolution is theoreticized by the higher male mutation rate because the male germline goes through many more rounds of cell division exposed more to methylation, regional and recombination effects (Li et al. 2002). Mutation rate can be 2-8 times higher in males than in females. Male-biased mutation causes Z-linked genes to 
evolve faster than autosomal genes, which in turn evolve faster than X-linked genes (Kirkpatrick \& Hall 2004). Sex chromosomes play a special role in speciation, especially in reinforcement processes under reduced effective gene flow between closely related incipient species. Haldan's rule refers to the preferential sterility or inviability of species hybrids of the heterogametic (Z-W, X-Y) sex. Z-W hybrids are often sterile or inviable whereas their ZZ siblings not. Large Z-effect (Xeffect) refers to the disproportionately large effect of $\mathrm{Z}$ chromosome versus autosomes in backcross genetic analysis of hybrid sterility and inviability (Presgraves 2008). Models predict that the evolution of sexual dimorphism is facilitated by sexlinkage for genes having different fitness effects in males and females. Such genes accumulate on $\mathrm{X}$ or $\mathrm{Z}$ chromosomes with non-additive allelic interactions.

There are factors leading to differences between sex chromosomes and autosomes in relation with speciation, all of which are linked to hemizygosity: (1) speed of evolution, (2) non-random accumulation of genes, (3) exposure of incompatible recessive genes in hybrids, (4) recombination rate (Qvarnström \& Bailey, 2009). Divergent selection favouring different phenotypes may occur during earliest stage of speciation in subdivided population to adapt to different environments in allopatry or disruptive selection favouring extreme phenotype competes over resource type in sympatry. Ecological divergence with few sex-linked genes, need to be coupled with evolution of sexual isolation to avoid homogenizing effect of gene flow in order to complete speciation process. New recessive beneficial mutations accumulate on sex chromosomes exposed to selection only in heterogametic sex having smaller effective population size than autosomes. X-linkage facilitates polymorphism: rare recessive mutation with large disadvantage in female and small advantage in male will not accumulate in autosome, but expressed in male. At the same time X-linkage can hinder polymorphism with its reduced effective population size (Gibson et al. 2001).

Genitalic traits are probably polygenic, coded by complex interactions of sex-limited loci and autosomes. Quantitative genetic studies are needed to understand patterns in the inheritance of parameres. Studies on rapid evolution of sex and reproduction related genes provide new approaches and facts to evolutionary biology: (1) importance of sexual selection, (2) rapid evolution of sexual traits, (3) high rate of DNA sequence divergence in genes of sexual function, (4) involvement of novel traits/genes in sexual function, (5) sex/non-sex dichotomy of gene pool affecting viability versus fertility (Singh \& Kulathinal 2000). X (and Z) chromosomes are enriched with genes associated with sex and reproduction and is a hotspot for sexually antagonistic fitness variation, harbouring $97 \%$ of its genome-wide total (Gibson et al. 2001).

Minor sex chromosomes. Minor sex determining, highly degenerative chromosomes persist in spite of the deleterious forces, possibly because of the presence of strong preservative forces powered by the essential role they play in sexspecific fitness. Possibly this power may also contribute to the rapid divergence of the speciation parameres in the sexual selection processes. There is only one female-determining $\mathrm{W}$ and one male-determining $\mathrm{Y}$ chromosome for every three $\mathrm{Z}$ or $\mathrm{X}$ chromosomes. Based on this abundance relation $\mathrm{W}$ and $\mathrm{Y}$ sex-limited chromosomes are termed minor sex-chromosomes and $\mathrm{Z}$ and $\mathrm{X}$ as major sex chromosomes (Mank 2012). Minor sex chromosomes are sometimes much smaller and degenerated with much reduced gene content. For instance just 27 distinct proteins are encoded by human $\mathrm{Y}$ chromosome, compared to more than 1000 found on human X. Moreover minor sex chromosomes often littered with repetitive sequences providing little evidence of functional utility and characterized also by transcriptional silencing. Nevertheless they can play important role in functions beyond sex determination (Mank 2012). Sex-limited Y or W chromosomes, having lost much of their genetic activity in the heterogametic sex (XY, ZW), trigger gene dosage compensation to ensure equal amount of gene products of X-linked or Z-linked loci in females and males. This dosage compensation is realised in Drosophila by higher rate of transcription in the $\mathrm{X}$ chromosome of male and in mammals only one of the two $\mathrm{X}$ chromosomes is active in female 
(Charlesworth 1996). Hemizygosity of sex-limited minor chromosomes may significantly acelerate and affect the divergence of sex-specific traits like parameres, through decreased effective population size and non-dominant recessive alleles. In various combinations and in very complex mechanism, positive selection and/or genetic drift may drive both faster- $Z$ and faster- $X$ in the formation of genitalic traits. Z-linked genes evolve up to $50 \%$ more rapidly than autosomal genes. The main source of faster- $Z$ evolution is the increased levels of genetic drift on the $Z$ chromosome due to sexual selection acting on male (Mank et al. 2010). This mechanism may have importance in the paramere divergence of the isolated caddisfly populations having very small effective population size. The faster-X effect is more sensitive to recessive mutations on the $\mathrm{X}$ directly exposed to selection in male (Sackton et al. 2014).

In Drosophila and mammals Y chromosomes encode genes key to male fertility and W-linked genes in birds are expressed in the ovary. Mating system and sexual selection acts differently on $\mathrm{W}$ and $\mathrm{Y}$ chromosomes. Under random mating the effective population size of $\mathrm{Z}$ is three-quarters as large as that for autosomes. However under sexual selection the mating is not random. Successful males mate multiple, reducing the number of males contributing to the next generation, decreasing further the effective population size of the $\mathrm{Z}$ chromosome relative to the autosomes. Decreasing the male proportion by sexual selection, the effective population size is reduced in $\mathrm{Y}$ compared to $\mathrm{W}$, therefore sex-specific selection pressure and genetic drift is stronger on $Y$ than on W chromosomes. Sex-limited portions of minor sex chromosome do not recombine with the homologous portion of major sex chromosomes locked into a complete linkage disequilibrium resulting in hitchhiking where selection processes fix beneficial mutations together with deleterious alleles at linked loci. Gene loss and suppression of recombination leads to chromosome degeneration. However strong male-specific selection pressures may help to maintain vital $\mathrm{Y}$ chromosome coding content against degenerative forces and similarly female-specific selection maintain W-linked genes. Traits encoded by genes on minor sex chromosomes are not subject to intralocus sexual conflict and therefore are more able to rapidly respond to sex-specific selection. Minor sex chromosome may interact epistatically with the entire genome and in spite of its limited coding content minor sex chromosomes has long reach even into quantitative trait loci (Mank 2012).

\section{Why neutral mtDNA marker is blind?}

Animal groups with limited morphological structures, such as the Lampetra genus, that possesses so few of the morphological characters (Mateus et al. 2013) or Protura where the taxonomy is based on subtle morphological differences, routine sequence data or even barcoding represent valuable source of information and mirrored by significant genetic distances (Resh et al. 2014). The population-genetic studies have been rather productive in the last decades based on the invention of polymerase chain reaction technology and on applying mitochondrial and nuclear neutral markers. Mitochondrial DNA has proved to be especially powerful for genealogical studies and microsatellite sequences for inferring population genetic structure and dynamics. However, both have important and unavoidable limitations (Zhang \& Hewitt 2003).

Conceptual limits. Individuals differ from each other by thousands to millions of DNA sequence variants. There are several conceptual problems in DNA taxonomy where the species boundaries are created by pairwise distances or by gene trees. Delimiting species with pairwise distances opposes any species concept that has ever been proposed (Meier 2008). (1) All specimens with sequences below a threshold value $(2-3$ per cent for COI) are considered conspecific. Threshold is highly taxa-dependent or even gene-dependent; therefore any average is highly arbitrary. Base substitution is very complex. (2) In separated or isolated populations synonymous or silent substitutions start to accumulate usually first having no significant change in phenotype as a rule, but can dramatically effect transcription, splicing, mRNA transport and translation, any of which could alter phenotype, rendering synonymous mutation nonsilent. Regulatory mechanisms of gene expression 
may have more potential to effect phenotype development than any measured DNA sequences. (3) Reproductive isolation usually acquired at a highly varying rate in all taxa destroying any fixed threshold of pairwise distances. (4) Speciation is not clockwise, exhibits wide ranges of distances. How is it possible to recognise species boundaries between closely related incipient species and to distinguish polymorphic species? (5) Using trees for determining species boundaries is especially problematic for sexually reproducing organisms. The relationship between populations and individuals are usually not hierarchical (Meier 2008). The maternal inheritance of mitochondria is not complete, and at best reflects only the maternal component of the population history.

Practical limits. In taxonomy, we are permanently faced to search new possibilities to enlarge our resolution power to find new and reliable traits to separate closely related incipient species. What kind of molecular genetic procedures would help us to confirm the taxonomic state of incipient caddisfly species? The routine „fingerprint” of mitochondrial DNA procedure ,confirmed” only those Chaetopteryx species which are anyhow, easily separated by traditional gross morphological structures (Kucinic et al. 2013). These taxa are the so called well recognised "good species". Easy to recognise them without any DNA sequences. Their species delimitation does not require fine structure analysis with very high sensitivity of fine resolution. Do the classical neutral markers really help us? It seems that mtDNA sequences are powerless to detect divergences in relation to any kind of phenotypic reality. These markers are really blind. At best their prediction potential is based simply on coinciding luck of micro (molecular genotypes) and macro (morphological phenotypes) shapes in differentiating among species of recently diverged or diverging clusters. Neutral markers could be almost fingerprinting sensitive. Often sorts completely in isolated lineages much long before the evolution of reproductive isolation or phenotypic divergence develops. These markers could qualify independently evolving units after a short allopatric phase and leading to taxonomic chaos. Especially in high mountain habitats where spatial or physical barrier to gene flow is abound. At the same time frequently fail to reflect species limits in sympatric and parapatric populations, as a result of incomplete lineage sorting, hybridisation and selective introgression (Tobias et al. 2010). We have documented that simple and cheap empirical procedure of fine structure analysis of the speciation trait has higher resolution power to detect divergences in these so called "cryptic" incipient phylogenetic species (Oláh et al. 2013a, $b, c, 2014)$. Low level of sequence divergence relative to morphological differentiation may be explained by rapid lineage sorting accelerated by sweepstakes recruitment, historical bottlenecks in population size, founder events, or natural selection, any of which could retard the accumulation of deep mtDNA lineages. This can lead to poorquality species delimitation because deeply divergent alleles can coexist within species due to introgression and incomplete lineage sorting, resulting in apparent species paraphyly.

Incomplete natural history of mitochondria. Surprising how several features of the intracellular genetic processes remained so poorly understood in animal mtDNA (Ballard \& Whitlock 2004). The favoured and widely applied neutral marker has proved to be not reliable enough. Especially those features seem more complex, which would be ideal for a molecular marker: low recombination rate, maternal clonality, neutrality, and clock-like nature. The unusual property of most animal mtDNA is that it does not undergo recombination (Birky 2001) However, extremely rapid concerted microevolution has been detected indicative of rapid gene conversion as a likely recombinational mechanism in animal mtDNA, although not interparental (Tatarenkov \& Avise 2007). A reappraisal review of the mitochondrial DNA as a marker of molecular diversity has questioned its maternal clonality, near-neutrality, and clock-like nature (Galtier et al. 2009). It is not always clonal, far from neutrally evolving, and certainly not clock-like. Its relevance as a witness of recent species and population history was also questioned and its usage for species delineation was critically evaluated.

Why neutral markers fail? There are several reason that mitochondrial DNA is not the ideal 
marker of molecular diversity, badly represent population history, perhaps intrinsically the worst molecular marker: (1) immune from neither recombination, positive selection nor erratic evolutionary rate; (2) its dense gene packing with no spacing introns sensitive to hitchhiking; (3) in linkage disequilibrium with selfish genetic element; (4) prone to genomic conflict; (5) located in active oxidative environment undergoes a complex mutation process, highly variable in space and time, with frequent rate heterogenity or mutateonal hot spots; (6) without histones has limited repair ability with relatively high mutationfixation rate; (7) evolve faster than nuclear genome, the rate of evolution is different for different regions; (8) becomes monophyletic more rapidly than a single nuclear gene and far more rapidly than a sample of several nuclear genes (Ballard \& Rand 2005); (9) phylogenies and population structure may not reflect those of the nuclear genome due to gender-biased migration or introgression; (10) subject to back mutation and substitution return to their original state; (11) parallel substitution, when mutations occur at the same site in independent lineages; (12) many of these confounding effects variable across taxa and the magnitude of variability is unknown; (13) does not reflect regulatory transcription and translation alterations or any epigenetic and developmental modifications in the phenotypic gene expression processes. These various evolutionary processes may either homogenize distinct species or produce balancing selection with high intraspecific diversity.

The trial to overcome limits of mitochondrial DNA marker with application of more neutral markers in order to improve statistics created more problems than solved. All the intrinsic limits, shortcomings, and problems have been multiplied proportionally with each new concatenation. The permanent fighting of alpha taxonomist with non-overlapping morphological and molecular trees became even more painful when more non-overlapping molecular trees were produced by additional markers. The epistemic empiricism of taxonomy faced the basic conflict of theoretical genomics. Finding consensus tree among contradicting sequences required more and more analytical models in a research algorithm already saturated with models: (1) models to collect samples, (2) models to produce primers, (3) models to amplify samples, (4) models to quantify samples, (5) models to generate sequences, (6) models to verify sequence identity, (7) models to create sequence alignment, (8) models to analyse sequences, (9) models of outgroup selection, (10) models to construct trees, (11) models to measure nodal support, (12) models to test topology, (13) models to search sequence consensus, (14) models to integrate multiple mapping, (15) pruning models to cloak and obfuscate inconvenient data (16) data-smoothing models to manipulate discordant, ambiguous data, (17) models of filtering algorithm to assess phylogenetic anomalies (18) models to simulate empiricism, (19) models to discuss reality, (20) models to rationalize further model buildings, and so on.

Far from diverging spots and evolving islands. Contradicting sequence trees are generated by neutral markers far from the selective genomic regions. Ambiguity is especially severe if markers are applied in young incipient species diverging only by small morphological structures, by behaviour or by any kind of speciation traits under directional selection. Neutral markers have no direct interactions with speciation genes or multigenes of quantitative trait loci under selective processes. Our knowledge is very limited about their possible relations to these isolated genomic spots, islands of speciation or even to „continent" of multiple differentiated loci that may decode the early stages of speciation (Michel et al. 2010). Any coincidences between routine neutral markers and speciation traits have no proper interpretation or explanation. Model created consensus could be an accident of luck, a spatially and temporally remote stochastic interaction or just a model forced fabrication. There has been no significant correlation found between neutral molecular marker diversity and the coefficient of variation for additive genetic variation of sexually selected traits among 20 species examined by Butlin \& Treganza (1998). In a meta-analysis on 71 data sets there was no significant correlation computed between molecular and quantitative 
measures of genetic variation of adaptive traits (Reed \& Frankham 2001). Similarly, high microsatellite genetic diversity fails to predict greater population resistance to extreme drought in 15 Banksia populations (He \& Lamont 2010). The presumption that neutral molecular markers can be used, as an indirect estimate of genetic variation, for polygenic traits in order to detect adaptive population divergence is intensively debated (McKay \& Latta 2002, Moss et al. 2003). Some evidence may indicate that mtDNA polymorphism can be maintained via selection on the joint mitochondrial-nuclear genotype (Dowling et al. 2008).

These various adaptive traits are under selective pressures similarly to the speciation traits on the phallic organ of diversifying caddisfly species. Genetic diversity measured via neutral markers is only surrogate for fitness and in best are indirect estimates or accidental luck for fitness-related traits. Neutral genetic markers, under the influence of stochastic processes of genetic drift and gene flow, often reveal local adaptation through their association with loci under selection. The real ability of selection to affect differentiation at neutral markers through hitchhiking is not yet fully understood. This effect probably extends over a very small chromosomal region not far beyond $5 \mathrm{~kb}$ around adaptive polymorphisms (Turner et al. 2010; Kolaczkowski et al. 2010). Unfortunately the reliance on this indirect path, on the use of neutral markers to infer the behaviour of selected loci, remains widespread, despite our ability to scan hundreds of loci with ease (Bierne et al. 2011). Moreover the indirect nature of inference is neglected, assumed to be similar to that of direct selection.

The frequently faced, gene tree and species tree" discrepancies is further complicated by genomic regions with different rates of evolution produced differently by various evolutionary mechanisms. Random genetic drift and population bottleneck produce genome-wide effect contrary to the regional effect of recombination and selection. A trial to ease this problem was to carry out analysis using multiple independent nuclear markers (Zhang \& Hewitt 2003). However conca- tenating several blind markers have produced more unresolved problems and now it is getting more obvious that molecular genetics has to move into a new phase of more intense searching the molecular basis of speciation traits and has to rely more upon the phenotypic traits.

\section{What sort of genetics could really help taxonomy?}

Without having a speciation trait, the most rapid, and cost-effective measures of genetic diversity are obtained from various assays of polymorphisms. However genetics is not taxonomy and genetic diversity is not a subject of alpha taxonomy! Barcoding or concatenating more neutral markers produces sequences far from adaptive genomic regions. Not known at all how far these sequences are from the contemporary evolutionary speciation processes. They give us less taxonomic information than the fine structure analysis of speciation trait, directly involved in the initial building of reproductive isolation. In case if speciation trait is available how molecular genetics can support the taxonomic research of closely related incipient species? How molecular genetics can help the taxonomic priority, other than phylogenetics, to collect, explore and describe biodiversity, the betrayed unknown 100 million taxa!

Genetics can help to detect speciation trait in a Holon context by molecular searching and confirming mechanisms in the coding-encoding-decoding triad. Find candidate genes which are responsible for the expression of the adaptive speciation traits! Find the genomic spot of the initial split! Find the genomic region of parameres, the phenotypic product of speciation! Several genomic tools are now available to answer these questions (Seehausen et al. 2014): (1) patterns of genomic divergence, (2) testing for signatures of introgression, (3) identifying signatures of selection by outlier analysis, (4) mapping genes of reproductive isolation, (5) genome scan of incipient species pairs along speciation continuum, (6) mapping links between divergent genomic regions and phenotypic traits, (7) admixture mapping of hybridizing taxa, (8) genomic cline analysis. 
However traits of genital morphology are mostly quantitative, encoded by multitude of genes of small effects with combined pleiotropy and epistasis on the quantitative trait loci. Major genes with large effect play a role in species differences only occasionally (Orr 2001). All the knowledge of quantitative trait loci on candidate genes has been accumulated mostly on model or domesticated organisms with very few relevance to the core mission of taxonomy! Moreover "knowledge" has been generated in highly artificial, controlled, and simplified laboratory environment far from the complexity of nature. Abiotic and biotic factors of selection can accelerate or constrain trait evolution. Quantitative genetic studies have still mostly neglected to isolate the effect of these factors. It is the time for manipulative field experiments and to identify speciation traits that are subject to selection (Anderson et al. 2014). Searching quantitative trait loci and candidate genes of such speciation traits in natural population is just started, but molecular tools are increasingly available to understand its genetics. (1) Quantitative trait loci mapping. (2) Mapping by pattern of gene expression. (3) Population genomics approach.

Mapping quantitative trait loci. Morphological variation of genital structures is often integrated and modular in nature along larger sets of traits. The shape and size of parameres may also vary in a coordinated and structured pattern. Sexual selection favours certain pattern of genetic variation of functionally related traits, and modular organisation may be created by correlated environmental responses or by homologous developmental pathways. Quantitative genetic parameters can be considered genetic descriptors of trait structure and modularity (Juenger et al. 2005). Concerted evolution of modular genetic architecture is canalized by mechanisms of parcellation, integration, pleiotropy, and differential epistasis, balanced by recombination, segregation, selection, drift and gene flow as well as realised through linkage disequilibrium. Reduced recombination maintains modules or trait combinations through linkage disequilibrium. Epistasis is a common mechanisms causing cryptic genetic variation for quantitative traits in natural popu- lations, but mapping of causal interacting variants is in its infancy (Mackay 2014).

Quantitative trait loci are stretches of DNA underlie a quantitative trait. Traditional mapping of quantitative trait loci is an important tool that breeders and geneticists routinely use to associate potential causal genes with phenotypes of interest. Pedigree information, inbred line crosses, recombinants are available and produced for linkage analysis to identify loci cosegregate with the trait.

Recombination during meiosis between homologous chromosomes may break gene linkages. The frequency of recombination of two genes in the same chromosome depends on the physical distance between them. Recombination rate between markers is an indication of the linkage: lower recombination suggests closer position in the construction of genetic maps. Mapping follows the co-segregation of polymorphic markers in structured experimental populations. Medium to high density maps is produced by applying few hundred to a few thousand markers. Finer mapping is accomplished by additional markers and by additional recombination events in the target area. Searching candidate genes which determine the performance trait in the mapped regions may be sought in the same species or in orthologous regions of model organisms for which complete genome information is available.

However these parameters in natural populations are usually not available. The traditional linkage analysis by family genealogy still can be productive, but alternative approach turns to natural populations, from linkage analysis to association studies (Goldstein \& Weale 2001). In association studies inter-individual variation is a prerequisite for effective mapping strategies to test the involvement of single nucleotide polymorphism (SNP) in affected (cases) and unaffected (control) individuals. Association or linkage disequilibrium mapping is a method of mapping quantitative trait loci in natural populations by scanning historic linkage disequilibrium to link phenotypes to genotypes. Genomewide set of markers is usually genotyped in a phenotypically variable population without 
genealogy and statistical associations are identified between markers and phenotypes (Seehausen et al. 2014). SNP frequencies differ if SNP influence the trait by biological causality or statistically correlated with a causal variant in non-random linkage disequilibrium. A systematic and exhaustive gene mapping based on linkage disequilibrium is a realistic prospect (Goldstein \& Weale 2001). There is an increasing possibility to use whole-genome linkage disequilibrium studies in order to map any trait genes. Such studies would employ a dense map of SNP to detect association between a marker and trait.

Mapping by pattern of gene expression. In the past, the expression of specific traits could only be measured by phenotype, for example by gross genital morphology in alpha taxonomy. Today transcriptome (total or specific subset of transcripts) and proteome (total or specific subset of proteins) can be directly investigated by highthroughput techniques in RNA and protein analysis, permitting to measure all genes expressed in a tissue and to decode networks of complex traits. Complete expression profiles, the transcript profiling of tissues or cell lines could be realised with microarray techniques. Gene expression profiling helps to understand the genome mechanisms which facilitate the identification of candidate genes or the entire pool of genes involved in expression of quantitative trait loci. The role of regulatory variation in complex traits is getting perspective also in disease risk analysis. Complex expression quantitative trait loci influence the expression level of genes (Albert \& Kruglyak 2015). Association studies between DNA sequence divergence and expression divergence demonstrated significant role of transcriptome in speciation (Wolf et al. 2010). Studies on transcriptome require expensive equipments, high technical skill, sophisticated data handling. Moreover RNA is very sensitive to degradation and is more difficult to quantify compared to DNA.

Mapping by population genomics. Population genomics approach is to detect, identify, and separate adaptive genes, locus specific effects of selection, mutation, assortative mating and recom- bination from genome-wide effects of genetic drift, gene flow and inbreeding. This is realized by adequate genetic markers including synonymous non-coding and non-synonymous coding nucleotide polymorphisms as well as using many mapped loci. Detection of selection signatures from genotyping to genome typing relies upon several principles. Neutral loci along the genome are similarly affected by genetic drift, demography, and evolutionary history of populations. Contrary, loci under selection often behave differently with ,outlier” patterns of variation: (1) loss of diversity; (2) increase of diversity under balanced selection; (3) linkage disequilibrium; (4) increased Fst/Gst outlier indices (outlier loci are genome locations or markers with variation extremely divergent from the rest of the genome); (5) selection influences linked markers also through genetic hitchhiking often detectable by genotyping.

Towards targets of selection. Inherent indirect probability of neutral markers to represent targets of selection has extorted searching non-neutral markers. These markers have direct ability to survey polymorphism on genomic scale in order to enable genome-wide scans for targets of natural selection. There are molecular markers associated with genes of known function (Liu \& Cordes 2004): (1) allosyme markers encode protein with known functions, (2) most restriction fragment length polymorphism were identified during analysis of known genes, (3) expressed sequence tags markers represent transcrips of genes, (4) some microsatellite markers associated with genes of known functions, (5) indel markers if located in genes, often discovered during genomic or transcriptomic sequencing projects. These markers are becoming important in studies of genetic linkage and quantitative trait loci mapping, and of candidate gene identification.

Decreasing cost, increasing potential of high throughput technologies and the availability of refined analytical models have already induced studies in natural populations of a few non-model organisms. Nanopore sensors using voltage of ionic current to drive molecules through a nanoscale biological pore has the ability to se- 
quence rapidly and reliably an entire genome for one thousand USD (Venkatesan \& Bashir 2011) characterising individual polynucleotide molecules (Kasianowicz et al. 1996). Ideas, principles, methods, and procedures of genomic research are radiating from model species to natural populations. The knowledge accumulated in Drosophila research on magic and sex-linked genes, as well as on genome scan and gene mapping of quantitative trait loci, expressing genital morphology is directly applicable to understand speciation processes in other groups of animals including diverging caddisfly populations. Transfer of genomic information from a map-rich group is an unexplored possibility to save much time, money and effort.

From neutral markers to entire genomic architecture of divergence. Producing more and more sequences of neutral markers is not enough to understand genetic processes in early stages of the adaptive, diverging speciation traits. To supply real molecular support for fine structure analysis of diverging traits, we have to apply genome scan and gene mapping of non-neutral adaptive region of genomic sequences or multigenic genomic regions of quantitative trait loci. Knowledge of genomic regions expressing speciation traits under sexual or natural selection processes would be more sensitive and would tell us more about where, why and how divergence takes place. The rapidly decreasing costs of high throughput sequencing already progressed to measure and analyse greater number of genes and populations. The first step to the targets of evolution was rather dubious and produced questionable results when routine barcoding of neutral mitochondrial DNA fingerprint was ,improved" by concatenated markers. In the second step, the focus of speciation research moved to search and identify individual speciation genes of reproductive isolation and/or ecological adaptation. But genes seldom work alone! Therefore the third step is directed to questioning ,genomic island”, ,continent” and the entire genomic architecture of divergence (Michel et al. 2010). Outlier analysis, entire genome scan, gene mapping and transcriptome research (the dark matter of evolutionary and medicine genomics!) are promising tools to analyse the structure and function of quantitative trait loci responsible to express phenotypes of speciation traits. This and not the barcoding would help us to understand phylogenetics and the speciation processes. But even this sort of molecular genetics would not help taxonomy to describe the mostly undiscovered biodiversity. Intrinsically, biodiversity description has priority over phylogenetics. We cannot target the phylogenetics of the undiscovered and undescribed, and especially of the never collected but killed species!

\section{MORPHOLOGICAL PART}

\section{Basal plate elongation in the vaginal sclerite complex}

At first glance the elongated vaginal sclerite complex present in Melampophylax females resembles to the vaginal sclerite elongation in the $A$. antennatus and $A$. hilaris subgroup of $A$. auricollis species group as well as in the $A$. uncatus species group of the genus Allogamus. In Allogamus the common duct of accessory glands with single internal tube is elongated to accommodate the fused single paramere during copulation (Oláh et al. 2014). In Melampophylax females the basal plate of the vaginal sclerite complex with two lateral and heavily sclerotized tubes is elongated to receive the two unfused parameres of plesiomorphic state.

To understand the differences we list the basic nine substructures composed of the vaginal sclerite complex (Oláh et al. 2013c, Oláh et al. 2014). (1) Vaginal sclerite plate itself on the dorsum of the membranous vagina and ventrum of the accessory gland duct; this basal plate integrates all the substructures. (2) Dorsal articulation sclerites, a variously sclerotized internal continuation of the supragenital plate (upper vulvar lip). (3) Ventral articulation sclerites attach the sclerite complex to the internal continuation of the lateral processes of the vulvar scales (lower vulvar lip), that is to the paired gonopods of segment IX. (4) Lateral joints of the upper and lower lips. Usually not, or less sclerotized, but sometimes enlarged and bloated by proliferation of hard tissue. (5) Wing sclerites with 
stretch function for vagina and with apodemic function anterad to receive vaginal muscles. (6) Hood-shaped junction sclerite holding and stretching the junction where the ducts of accessory gland and ovarium meet as well as separating accessory duct from spermathecal duct. (7) Spermathecal process receiving ductus spermathecae and forming frequently a longitudinal keel on the ventrum of the vaginal sclerite. (8) Bursal sclerite receiving ductus bursae that opens between the spermathecal process and the common oviduct at the anteriomesal margin of the vaginal sclerite. (9) Anterior apodemes appear as the anteriormost lateral extension of the vaginal plate separated by the mesal bursal sclerite. Receive muscles and frames the space for the bursal sclerite ensuring to receive ductus bursae.

It seems that in the formation of plate elongation of the genus Melampophylax the articulation sclerites and their articulations participate. It requires detailed anatomical and histological studies to understand exactly, which structural units participate, and to what extent in the elongation. However for our alpha taxonomy the very position of bursal sclerite opening at the tip of the elongation is a direct indication of plate elongation. The hood-shaped junction sclerite as well as the spermathecal process remained on their original plesiomorphic posterior position.

\section{MATERIAL AND METHODS}

Put together! Population thinking in the new taxonomy requires more elaborated field collecting strategies. To collect many specimens from many populations are the prime target of any research project aimed to find the first signatures of reproductive isolation, to search species boundaries, to delimit closely related incipient taxa, and to recognize the young phylogenetic species. Biodiversity research and conservation are badly limited by the lack of population field sampling, which is expensive. Staggering in the deprived discipline of taxonomy and suffering the lack of adequate collecting we have been forced to outline the principles and practice of cooperation how to put together what we have (Oláh et al. 2013c). There are some historical materials scattered in museum, university, and private collections. Taxonomy has to survive somehow the pharisaic European science and environmental policies: speaking nice and killing biodiversity twice: with economy and pharisaism! We have laboured an idea of cooperation how to realise comprehensive studies when funding is removed from taxonomy to "modern" disciplines of genetics, ecology, and conservation and no resource remained available even for adequate population sampling.

Theories, algorithms, methods, and procedures of population sampling are the basis of the New Taxonomy. Today under the present course of resource disposing policy we have to rely upon caddisfly specimens already collected in various research projects and deposited in various collections. If money limits our efforts in alpha taxonomy we have to put together what we have. To bring together these scattered specimens we need a specialist who is interested in that particular species complex. He will initiate and organise this collective effort. We have to understand clearly that the collected, sorted, and determined material incorporates already significant scientific work and has high primary value for such a joint surveys. Adequate population sampling is a key component of any fine structure analysis of phenotypic speciation traits. Therefore we practice that colleagues who contribute to the survey with their specimens and agree with the final findings become co-author of the paper and/or of the species automatically. It was a great challenge and required sustained effort to bring together all the necessary specimens to revise these limnephilid taxa, including new collecting trips by many of the authors between the years of 2009 and 2014.

Clearing, cleaning, and drawing procedures. This study is based on animals preserved in $70-80 \%$ alcohol. In order to observe morphological details in the genitalia, the entire or only the terminal two-three segments of abdomen were removed and placed in a small glass beaker of $25 \mathrm{~cm}^{3}$ with $10 \% \mathrm{KOH}$ solution and boiled during 5-15 minutes for digestion above a spirit burner. The duration of the treatment is adjusted individually to the effectiveness of clearing process which depends on the species or even on the nutritive state of tissues or on the physiological condition of the specimens. The process of digestion can be easily followed by transparency. The dissolution rate of the soft tissues, the clearing transparency is visible to naked eye. The clearing process and time are so much taxon, size, age, sex, and nutrition state specific that automatic hot plate or bath clearing is not practical. The digested abdomen was subsequently transferred to distilled water and the macerated tissue was removed mechanically in patient cleaning process by fine tipped forceps and needles. The internal vaginal sclerite complex was 
exposed to clear view by cutting windows into the dorsum and left pleuron with fine scissor. The cleared and cleaned abdomen was transferred to $80 \%$ ethyl alcohol, and to glycerine for examination under microscope. Different sized pins modified to supporting ring bottom was introduced into the abdomen and used to hold and stabilise the genitalia in lateral, dorsal, and ventral position for drawing. However, the plane of view is never perfect and we made no special procedures of grid, matrix, or reflection to produce absolute mirror symmetry of the drawings. Instead, the genital structures are drawn exactly as seen in the microscope. However setae are represented only by their alveoli and moreover their density is only symbolic. If essential the setal length or setal shape are presented by drawing a single or a few setae only. The genital structure was traced by pencil on white paper using a drawing tube mounted on a WILD M3Z microscope at between 260x and 416x magnification. Final illustrations were prepared by enlarging the original pencil drawings and re-drawn on transparent paper by Black India Ink.

Terminology. We used our functional appendicular terminology and not the conventional anatomical directional terminology to describe the genital structures in species description (Oláh \& Johanson 2008). Species descriptions were standardized to ensure consistently formatted and comparable description in general accord with Evenhuis's (2007) template principle. We have standardized also the terminology to describe space extensions of variously formed structural elements. The following terms were used to qualify the dimensions and extensions of genital structural elements: (1) short or long for length dimension on the longitudinal direction of coronal plane along the anteroposterior axis; (2) low or high (traditionally shallow or deep especially for excisions) for height dimension on the vertical direction of the sagittal plane along the dorsoventral axis and (3) narrow or wide (broad) on the lateral direction of the transversal plane along the mediolateral or left-right axis. The three dimensional Cartesian coordinate system provides theoretical possibility to quantify by measurements the three physical dimensions of length, width, and height of each structural element. However this quantification is used very seldom in species description. Here we quantify only the length of forewing.

\section{Depositories.}

Civic Natural Science Museum “E. Caffi”, Bergamo, Italy (CNSMB)
Cianficconi Collection, Italy (CC), deposited at the Civic Natural Science Museum "E. Caffi" of Bergamo, Italy

Coppa Private Collection, France (CPC)

Departamento de Zoología Facultad de Ciencias. Universidad de Granada, Spain (DZFCUG).

Hungarian Natural History Museum, Budapest (HNHM)

National Academy of Sciences of Ukraine, State Museum of Natural History, Lviv (SMNHL)

National Museum, Prague, Czech Republic (NMPC)

Oláh Private Collection, under national protection by the Hungarian Natural History Museum (OPC).

Polish Academy of Sciences. Natural History Museum of the Institute of Systematics and Evolution of Animals, Krakow, Poland (NHM-ISEA)

The Manchester Museum, University of Manchester, England (MMUE)

The Natural History Museum, London (NHM)

Museum für Naturkunde, Berlin, Germany (MFN)

\section{TAXONOMY}

\section{Family Limnephilidae Kolenati, 1848}

\section{Subfamily Drusinae Banks, 1916}

The genital architecture of the subfamily is rather uniform. Therefore, most of the genera are artificial construct established primarily by structures of sexual dimorphisms or secondary modifications on forewing or hindwing. Schmid (1955) has introduced the term of neoformation for these unusual structural modifications into the trichopterology. These neoformations appear very frequently as secondary sexual traits and develop usually on palps, scapes, legs and on wings in the form of setal bunch (pencil of hairs of McLachlan, accommodated in a pouch), scaly formations. Neoformations may also evolve as bizarre shape transformations of any structures. They are less common in primitive lineages and more common in specialized lineages. Bizarre modifycations appear throughout the caddisfly taxa. Most of these unusual structures are developed to facilitate certain forms of sensory functions in sexual selection through stochastic speciation processes including mutations, genetic drift, and gene flow. The first discoveries of these secondary oddities have initiated the proliferation of 
genera in hydroptilids and lepidostomatids (Mosely 1934, 1937, 1939). We have found such modifications, present or absent coupled with rather similar genital structures (Oláh \& Johanson 2011, Oláh \& Flint 2012). This finding suggests that these bizarre modifications are species specific rather than genus specific. The taxonomic value of sexual dimorphism and secondary modification was downgraded in Lepidostomatidae family and 25 genera have been synonymized with the genus Lepidostoma (Weaver 2002). Such a taxonomic study is badly needed also in Drusinae subfamily.

\section{Genus Drusus Stephens, 1837}

The genus is highly homogenous, similarly to the entire subfamily. The first species grouping of Drusus genus was elaborated by Schmid (1956). $\mathrm{He}$ has established six species groups based almost exclusively on the shape and position of the paraproct. However he has emphasized that his species grouping in Drusus genus, as well as the six genera revised in the subfamily, are only artificial constructs. Schmid's basic conclusion was confirmed, but no new grouping idea or system was suggested, in a recent cooperative complex study (Pauls et al. 2008), based on larval morphology, larval feeding strategy, and sequence data from two mitochondrial loci and one nuclear gene. A more comprehensive and complex species grouping in the Drusus genus as well as genus downgrading evaluation in the Drusinae subfamily is required applying several morphological traits of varying diversity, stability rates and of different splitting ages. In the Drusinae subfamily and in the Drusus genus the paraprocts and the parameres seem sensitive enough to detect divergences among closely related incipient species.

\section{Drusus mixtus species group}

This rather heterogeneous species group was erected by Schmid (1956) and he has characterized the group by paraproctal dorsal branch (Oláh et al. 2014: internal, apical and horizontal branch) having sagittal plate-form, mostly fused mesad and deeply excised between the apical hook and basal pointed denticulate formation in lateral view; paraproct ventral branch (body of segment $\mathrm{X}$ or inferior branch of paraproct by Schmid, or basal, vertical branch) less developed and open ventrad. The group exhibits some tendency also to a reduced body of paraproctal dorsal branch as visible in caudal and dorsal view. The paraproct ventral branch is especially reduced, open ventrad without any sclerotized transversal section.

However, the reduced size of the paraproct and especially the loss of sclerotized transversal section of the ventral branch is only a tendency. The Drusus mixtus species group needs a revision; probably it is composed of several lineages. The mesal fusion of the paraproctal dorsal branch is not general. $D$. trifidus, $D$. brunneus have the paraproctal ventral branch entirely and $D$. improvisus partially closed, not open; $D$. mixtus, $D$. biguttatus and D. spelaeus have open ventral branch, but not widely expanded laterad. The Drusus bolivari new species complex has open and widely expanded ventral paraproctal branches.

\section{Drusus bolivari new species complex}

Drusus bolivari was described by McLachlan (1876) from a single male specimen collected in the Guadarrama Mts., Spain. Later McLachlan (1884) described Drusus estrellensis from several male and female specimens collected in the Estrella Mts., Portugal. Schmid (1952a) has collected and identified Drusus bolivari specimens from the Gredos Mts., Spain. Later he has synonymized $D$. estrellensis with $D$. bolivari without examining the holotype of $D$. bolivari deposited in The Natural History Museum, London (Schmid 1956). He has prepared male and female drawings for $D$. bolivari from specimens collected by himself in the Gredos Mts. Malicky (1983) has included Schmid's drawings for $D$. bolivari in his Atlas of European Trichoptera. In his second edition of the Atlas of European Trichoptera (2004) Malicky has prepared his own drawings for $D$. bolivari from a male specimen sent by Prof. Gonzales. This specimen was collected in Galicia, Spain.

McLachlan's original drawings are very precise and detailed enough; therefore finally we 
have three comparable and completely different drawings for $D$. bolivari. Each drawing was prepared from specimens originated from different mountain ranges. We have collected and examined specimens from Ancares Mts., Demanda Mts., Baetic Mts, Pyrénées Mts. and examined also the type materials of $D$. bolivari from Quadarrama Mts. and D. estrellensis from Estrella Mts., as well as Schmid's male and female specimens collected in Gredos Mts. Based on fine structure analysis we have recognised that specimens from all mountain ranges are separate species. What was determined earlier as a single species of Drusus bolivari it actually represents an entire species complex with 8 species. However, more incipient species of this species complex may inhabit various isolated mountains ranges in the Ibearian Peninsula.

The species complex is characterized by reduced paraproct. Paraproct dorsal branch is reduced laterad almost into a fused plate. The reduced development of the paraproctal ventral branch without sclerotized transversal section is very pronounced and the lateral section of the ventral branch widely expanded laterad. Mesal fusion of the paraproctal dorsal branch is present and definite. Hook formation on the paraproctal dorsal branch in lateral view variously developed.

To distinguish between taxa of this species complex we rely upon the shape of paraproct and the setal pattern of the paramere both in left lateral view. In Drusus genus we find the paraproct rather divergent among taxa and stable both inside and between populations. Paraproct may have an important direct function in copulatory processes. The setal pattern of the paramere is stable with a range of variation. Variability is caused by two types of artefacts. First type of variation is the result of functional injuries, distortions, or even damages accumulated during copulatory actions. The actual setal pattern depends on whether the parameres of the specimens what we examine was collected before or after the copulation. The second source of variation is subjective. On the high resolution a tiny alteration in the view of examination significantly changes the pattern of this fine structure. Such subjective source of variation is very pronounced also in the dorsal or caudal view of the paraproct. These views are unstable, very difficult to reproduce similar drawings even from the same specimen. Moreover the paraproct in caudal view is practically not visible in front of the black spinulose area of tergit VIII.

\section{Drusus carmenae Oláh, sp. nov.}

(Figures 1-5, 6-8)

Diagnosis. Most close to D. grafi sp. nov., but differs by having spinulose area on tergit VIII differently shaped; cerci short and high quadratic, not low and long triangular; paraproct dorsal branch straight and blunt not with pointed slightly recurving dorsoapical region in lateral view and more bifid in apical view; paraproct ventral branch with robust lateral section, not thin; gonopods short and robust not elongated slender; paramere with 3-4 modified setae, not with 3 spine-like setae.

Material examined. Holotype. Spain, Nacimiento R. Guadalquivir, Sierra de Cazorla (Jaén), $37.902273^{\circ} \mathrm{N}, 2.937723$ ${ }^{\circ}$ E, 1378 m, 9. X. 2009, leg. C. Zamora-Muñoz, (1 male, DZFCUG). Allotype. Same as holotype (1 female, DZFCUG). Paratypes. Same as holotype (1 male, 1 female, DZFCUG; 1 male, 1 female, OPC). Spain, Nacimiento R. Castril, Sierra de Castril (Granada), $37.895773^{\circ} \mathrm{N}, 2.747502$ ${ }^{\circ} \mathrm{E}, 1220$ m, 28. XI. 2001, leg. C. Zamora-Muñoz, (1 male, DZFCUG, 1 male, OPC).

Description. The new species with light brown, almost stramineous body, and wings. Head and thoracic sclerites darker brown; setal warts, both on the head and thorax, and the palps, antennae and legs lighter brown. Spur formula $1,2,3$ both in male and female. Forewing length $10 \mathrm{~mm}$.

Male genitalia. Apical spinulose area on tergit VIII slightly trilobed both in lateral and dorsal view. Cerci high and low quadratic. Paraproct dorsal branch with straight and blunt dorsoapical region, simple digitate in dorsal view, bifid in apical view; paraproct ventral branch without sclerotized ventrum, widely expanded laterad, lateral section robust. Gonopods short and high in lateral view and mesad turning in ventral view. Parameres with 3-4 modified setae. 


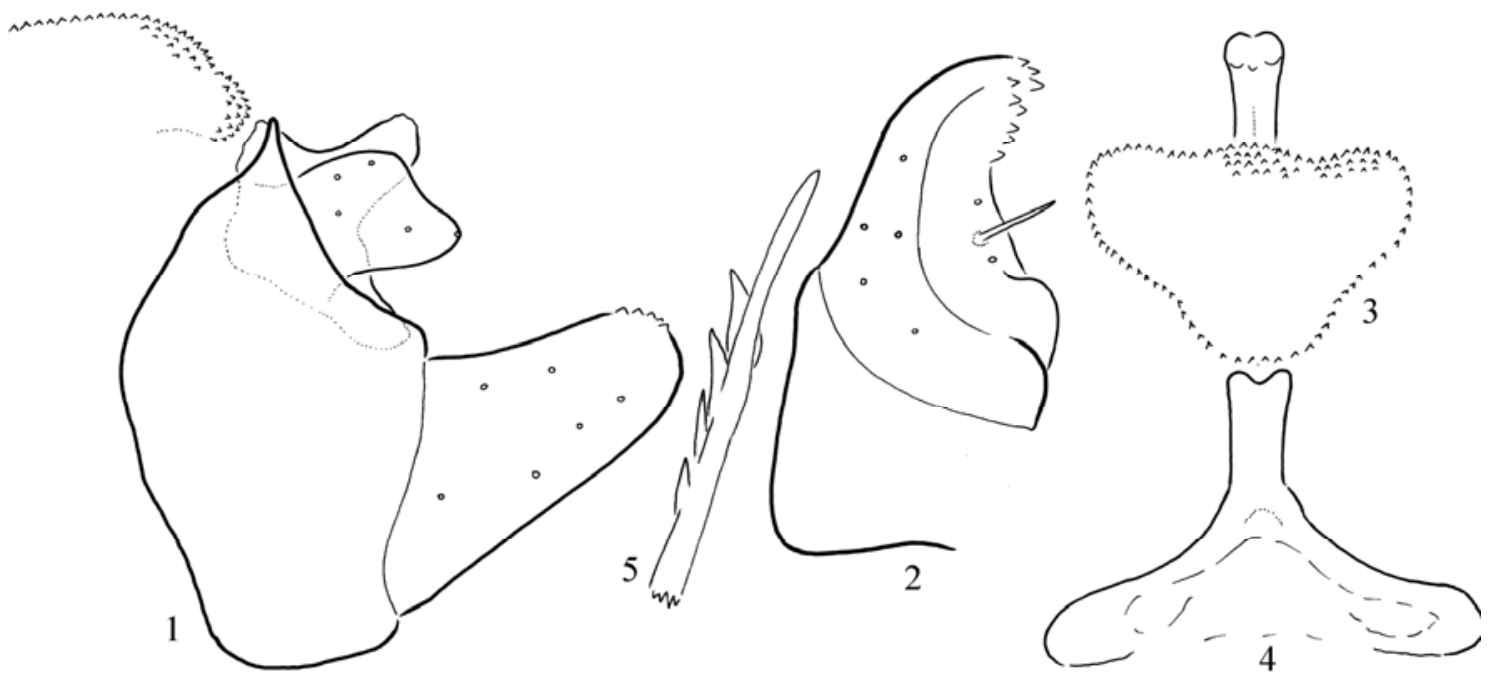

Figures 1-5. Drusus carmenae Oláh sp. nov. 1 = male genitalia in left lateral view, $2=$ left gonopod in ventral view, 3 = paraproct dorsal branch and spinulose pattern on tergite VIII in dorsal view, 4 = paraproct dorsal and ventral branches in caudal view, 5 = apical section of left paramere in lateral view.

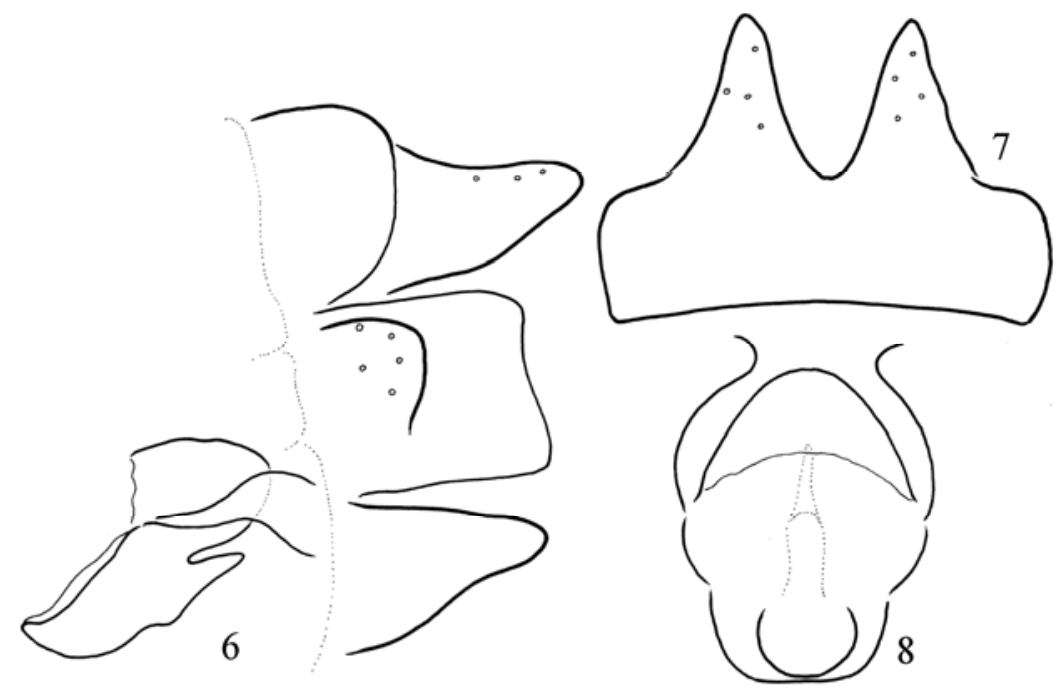

Figures 6-8. Drusus carmenae Oláh, sp. nov. 6 = female genitalia with dorsal vaginal sclerite complex in left lateral view, 7 = fused tergite IX and $\mathrm{X}$ in dorsal view, $8=$ dorsal vaginal sclerite complex in dorsal view.

Female genitalia. Tergum IX forms a short tube open ventrally; tergum $\mathrm{X}$ deeply bilobed in dorsal view. Lateral setose lobe, the sternite of segment IX rounded. Supragenital plate of sternum X well-developed and quadrangular in lateral view. Median lobe of the vulvar scale (lower vaginal lip) well developed. Vaginal chamber me- dium sized reaching almost to the anterior margin of sternite VIII, vaginal sclerite complex oviform in dorsal view.

Etymology. We dedicated this species to the collector, Dr. Carmen Zamora-Muñoz, to appreciate her significant contribution to the knowledge of Trichoptera. 


\section{Drusus bolivari (McLachlan, 1876)}

(Figure 9)

Catadice bolivari McLachlan, 1876: 40-41. Described from a single male specimen collected in Spain (Sierra de Guadarrama, Navacerrada Pass, leg. Bolivar). Catadice was synonymised with Drusus by Mosely 1933: 499.

Drusus bolivari (McLachlan, 1876): Schmid 1952a: 680. Male and female specimens collected in Gredos Mts. Spain. Misidentification! Described here as Drusus gredosensis sp. nov.

Drusus bolivari (McLachlan, 1876): Schmid 1956: 51-52. synonymised $D$. estrellensis (McLachlan, 1884) with $D$. bolivari (McLachlan, 1876).

Drusus bolivari (McLachlan, 1876): Malicky 1983: 165. Misidentification. Redescribed here as Drusus gredosensis sp. nov.

Drusus bolivari (McLachlan, 1876): Drawings by Malicky 2004: 197. Misidentification! Described here as Drusus gonzalezi sp. nov.

Diagnosis. Drusus bolivari, the name bearing species of the complex has unique paraproct, its dorsal branch is almost horizontal and its dorsoapical region bifid, apex truncated, upper margin excised before apex in lateral view. Unfortunately the paramere not fully visible on the single male holotype genitalia embedded in a permanent slide preparate.

Material examined. Holotype, male. Deposited in The Natural History Museum, London. Cleared abdomen and rest of the body without wings are embedded in permanent slide preparate with two labels: (1) red-framed label: Catadice Bolivari McLach. Det. McLachlan TYPE. C/B. (2) black double-line framed label: Navacerrada, Spain Mclachlan Coll. B. M. 1938-674, BMNH(E) \# 251071.

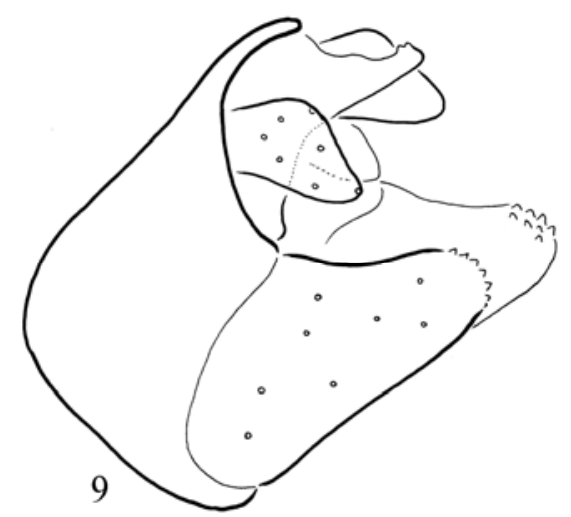

Figure 9. Drusus bolivari (McLachlan, 1876). Male genitalia in slightly left caudolateral view drawn from the permanent slide preparate of the holotype.

\section{Drusus estrellensis (McLachlan, 1884) stat. restit.}

\author{
(Figures 10-14, 15-17)
}

Catadice estrellensis McLachlan, 1884: 13. Catadice synonymised with Drusus by Mosely 1933: 499.

Drusus bolivari (McLachlan, 1876): Synonymised with $D$. bolivari by Schmid 1956: 52. Misidentification.

Diagnosis. Species described from Portugal, Estrella Mts. The lateral shape of paraproct is most close to $D$. pyrenensis, but more robust; differs also by having higher number (6-7) of modified setae on the paramere, not less number (3-5), cerci regular quadratic, gonopods short and high, not long and low in lateral view.

Material examined. Holotype male. Deposited in The Natural History Museum, London. Cleared abdomen embedded in permanent slide preparate with two labels: (1) redframed label: Catadice estrellensis McL. § TYPE. C/B. (2) black double-line framed label: Portugal, Sabagueiro 5/6/ 1880 A.E.Eaton McLachlan Coll. 1938-674, BMNH(E) \# 251069. Type series: Catadice estrellensis McL. ते TYPE. With seven labels: (1) brown small oblong: Type; (2) pink oblong handwritten: Catadice estrellensis McL.; (3) gray oblong typed: Catadice estrellensis, McLach. Det. McLachlan; (4) blue oblong: McLachlan Coll. B. M. 1938-674; (5) yellow short oblong: Eaton. Portugal. 1880; (6) white oblong with code: $\mathrm{BMNH}(\mathrm{E}): 1253659$; (7) yellowish rounded one side: b. 5. VI.80, other side: Sabugueiro, 5/6. Catadice estrellensis McL. ठิ TYPE. With seven labels: (1) red small oblong: Type; (2) pink oblong handwritten: Catadice estrellensis McL.; (3) gray oblong typed: Catadice estrellensis, McLach. Det. McLachlan; (4) blue oblong: McLachlan Coll. B. M. 1938-674; (5) yellow short oblong: Eaton. Portugal. 1880; (6) white oblong with code: BMNH(E):1253660; (7) yellowish rounded one side: b. 5. VI.80, other side: Sabugueiro, 5/6. Catadice estrellensis McL. + TYPE. With seven labels: (1) brown small oblong: Type; (2) pink oblong handwritten: Catadice estrellensis McL.; (3) gray oblong typed: Catadice estrellensis, McLach. Det. McLachlan; (4) blue oblong: McLachlan Coll. B. M. 1938-674; (5) yellow short onlong: Eaton. Portugal. 1880; (6) white oblong with code: $\mathrm{BMNH}(\mathrm{E}): 1253661 ;$ (7) yellowish rounded one side: b. 5. VI.80, other side: Sabugueiro, 5/6. Catadice estrellensis McL. + TYPE. With seven labels: (1) brown small oblong: Type; (2) pink oblong handwritten: Catadice estrellensis McL.; (3) gray oblong typed: Catadice estrellensis, McLach. Det. McLachlan; (4) blue oblong: McLachlan Coll. B. M. 1938-674; (5) yellow short oblong: Eaton. Portugal. 1880; (6) white oblong with code: BMNH(E):1253662; (7) yellowish rounded one side: b. 5. VI.80, other side: Sabugueiro, 5/6. 


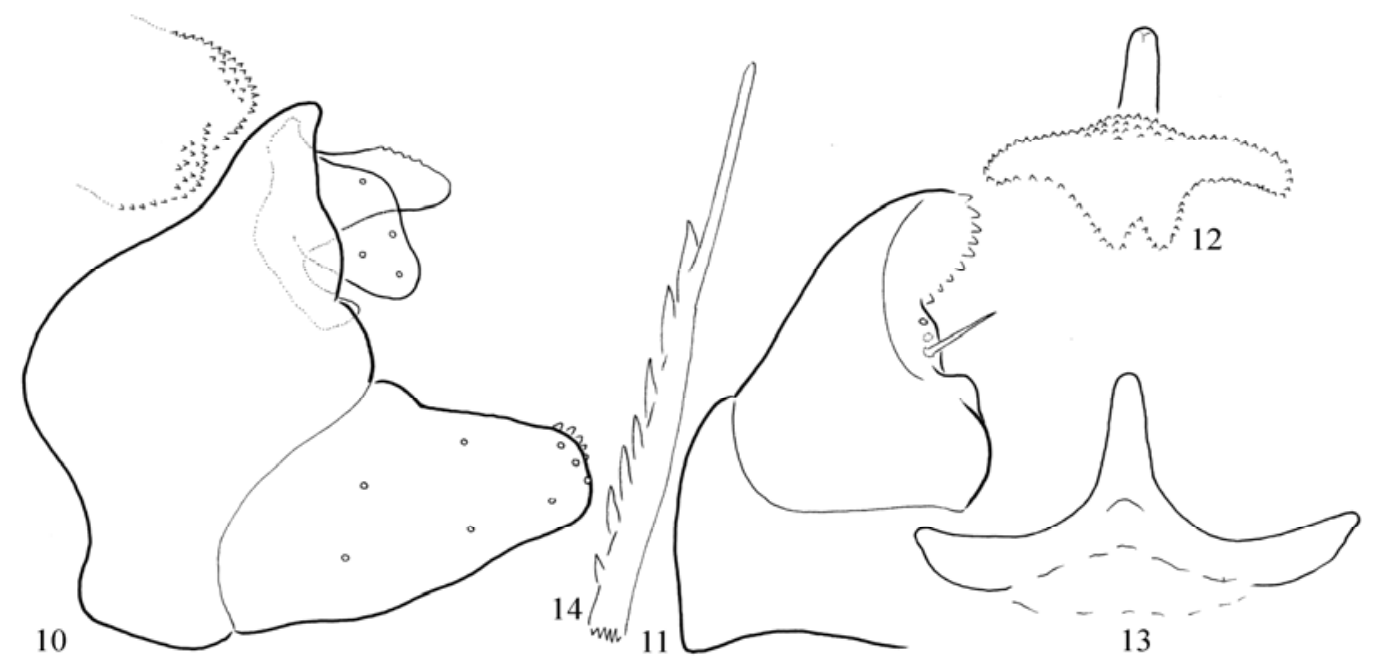

Figures 10-14. Drusus estrellensis (McLachlan, 1884). 10 = male genitalia in left lateral view, $11=$ left gonopod in ventral view, 12 = paraproct dorsal branch and spinulose pattern on tergite VIII in dorsal view, 13 = paraproct dorsal and ventral branches in caudal view, 14 = apical section of left paramere in lateral view.
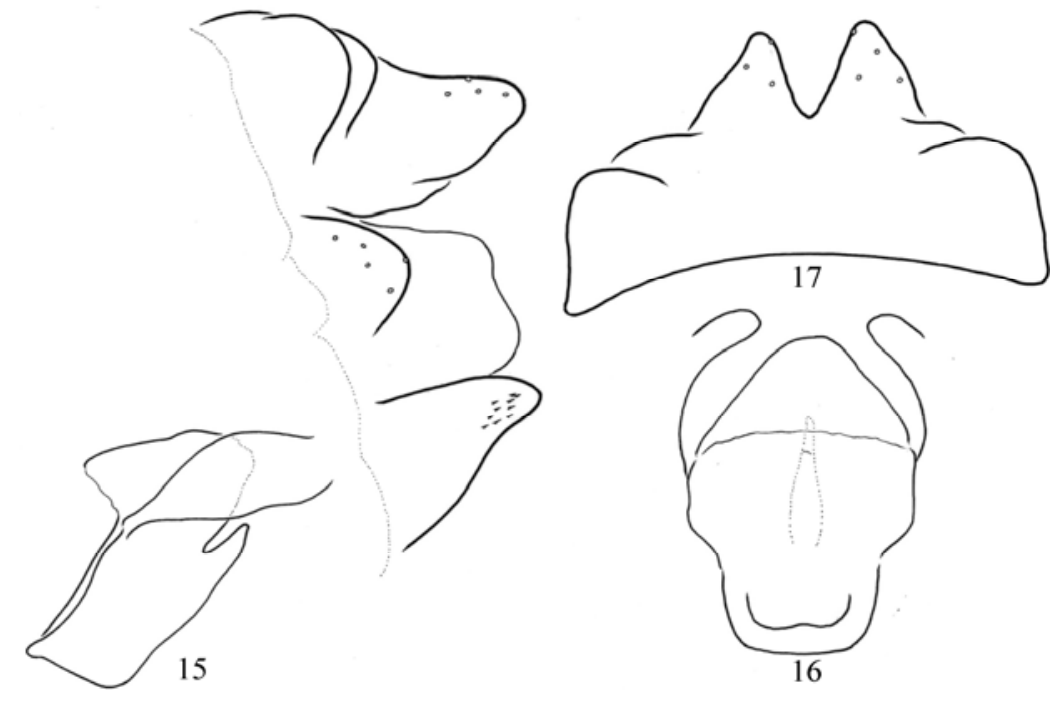

Figures 15-17. Drusus estrellensis (McLachlan, 1884). 15 = female genitalia with dorsal vaginal sclerite complex in left lateral view, 16 = fused tergite IX and $\mathrm{X}$ in dorsal view, $17=$ dorsal vaginal sclerite complex in dorsal view.

\section{Drusus gonzalezi Oláh, sp. nov.}

(Figures 18-22, 23-25)

Drusus bolivari (McLachlan, 1876): Drawings by Malicky 2004: 197. Misidentification!

Diagnosis. The drawings of this species was published under the name Drusus bolivari Mc-
Lachlan (Malicky 2004), but differs from it by having simple straight and long triangular shape of paraproct dorsal branch in lateral view, not bifid and excised dorsoapicad; gonopods with tapering apex, not blunt. It is most close to Drusus carmenae sp. nov., but differs by having narrowing apex of the paraproct dorsal branch not blunt in lateral view, bellied laterad, not straight in 
caudal view; parameres with six spine-like setae.

Material examined. Holotype. Spain, NW Spain, Sierra de Ancares, Lugo, Rio de la Vara, Rio da Vara, N42 $48^{\circ}$ 10.74" W6 53'19.90”, 1230m, 14.VII.1984, leg. Marcos A. Gonzalez (1 male, OPC). Allotype. Same as holotype (1 female, OPC).

Male genitalia. Apical spinulose area on tergit VIII very distinctly trilobed with long anterior mesal band. Cerci low and long subtriangular. Paraproct dorsal branch straight and long triangular in lateral view, simple digitate in dorsal view, and bellied in caudal view; paraproct ventral branch without sclerotized ventrum, widely expanded laterad. Gonopods elongated slender in lateral view and mesad turning in ventral view.
Female genitalia. Tergum IX forms a short tube open ventrally; tergum $\mathrm{X}$ deeply bilobed in dorsal view. Lateral setose lobe, the sternite of segment IX rounded. Supragenital plate of sternum X well-developed, rounded subquadrangular in lateral view. Median lobe of the vulvar scale (lower vaginal lip) well developed. Vaginal chamber medium sized reaching almost to the anterior margin of sternite VIII; vaginal sclerite complex oviform.

Etymology. We dedicated this species to the collector, Professor Marcos A. Gonzalez to appreciate his significant contribution to the knowledge of European Trichoptera.

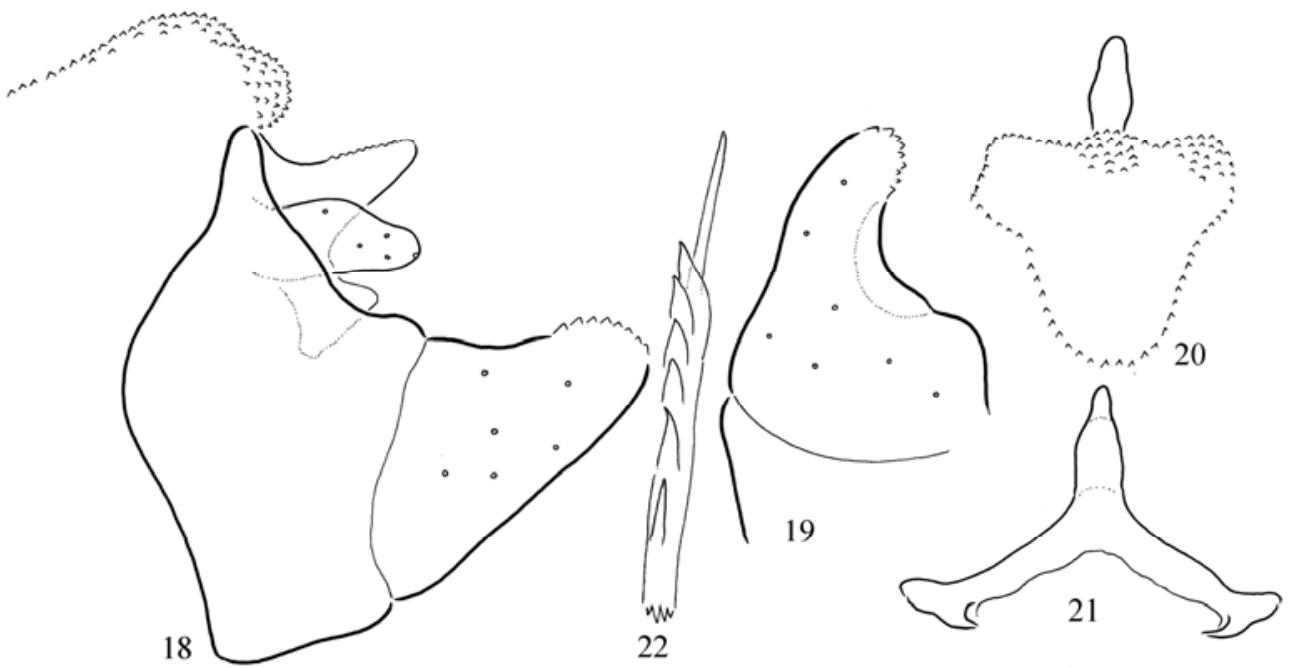

Figures 18-22. Drusus gonzalezi Oláh, sp. nov. $18=$ male genitalia in left lateral view, $19=$ left gonopod in ventral view, 20 = paraproct dorsal branch and spinulose pattern on tergite VIII in dorsal view, 21 = paraproct dorsal and ventral branches in caudal view. 22 = apical section of left paramere in lateral view.

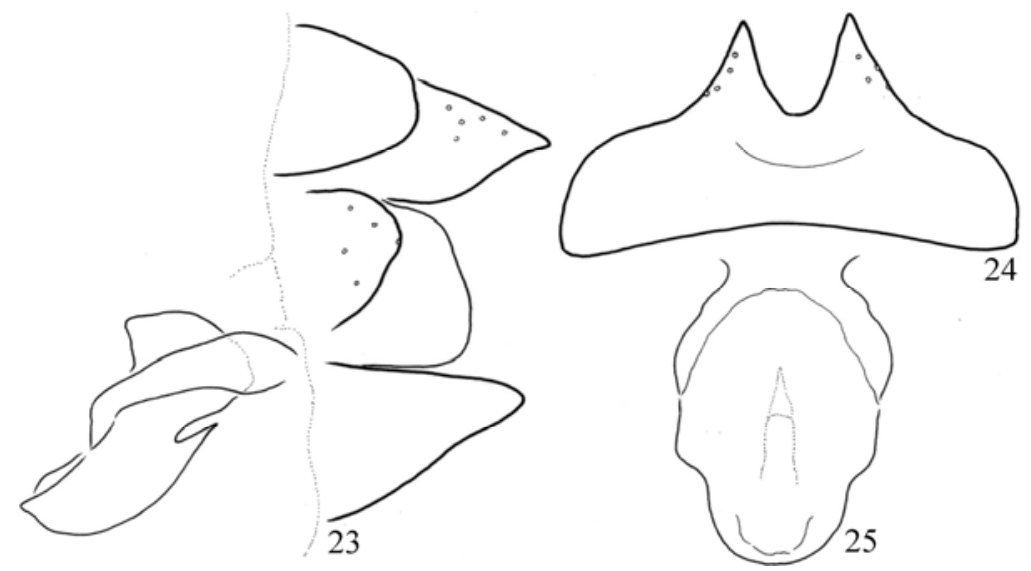

Figures 23-25. Drusus gonzalezi Oláh, sp. nov. 23 = female genitalia with dorsal vaginal sclerite complex in left lateral view, $24=$ fused tergite IX and $\mathrm{X}$ in dorsal view, 25 = dorsal vaginal sclerite complex in dorsal view. 


\section{Drusus grafi Oláh, sp. nov.}

(Figures 26-30, 31-33)

Diagnosis. Most close to D. carmenae sp. nov., but differs by having spinulose area on tergit VIII differently shaped; cerci low and long triangular, not short and high quadratic; paraproct dorsal branch with pointed slightly recurving dorsoapical region, not straight and blunt in lateral view and less bifid in apical view; paraproct ventral branch with thin lateral section, not robust; gonopods elongated slender, not short and robust; paramere with 3 modified setae, not with 3-4 spine-like setae.

Material examined. Holotype. Spain, Sierra de la Demanda, near Ezcaray, $42.255785^{\circ} \mathrm{N}, 2.978349^{\circ} \mathrm{E} .1371 \mathrm{~m}, 15$. VI. 2013 leg. W. Graf (1 male, OPC). Allotype. Same as holotype. Paratype. Same as holotype (1 male, OPC).

Description. A new species with brown body and wings. Head and thoracic sclerites darker brown; setal warts both on the head and thorax, as well as palps, antennae and legs lighter brown. Spur formula 1,2,3. Forewing length $12 \mathrm{~mm}$.

Male genitalia. Apical spinulose area on tergit
VIII slightly trilobed in lateral view. Cerci very low, long triangular. Paraproctal dorsal branch with poited dorsoapical corner in lateral view, simple digitate in dorsal view, slightly bifid in apical view; paraproct ventral branch without sclerotized ventrum, widely expanded laterad, lateral section thin. Gonopods elongated slender in lateral view and mesad turning in ventral view. Parameres with 3 modified setae both at holotype and paratype.

Female genitalia. Tergum IX forms a short tube open ventrally; tergum $\mathrm{X}$ deeply bilobed in dorsal view with pointed apices. Lateral setose lobe, the sternite of segment IX elongated. Supragenital plate of sternum $\mathrm{X}$ well-developed, quadrangular in lateral view. Median lobe of the vulvar scale (lower vaginal lip) well developed. Vaginal chamber medium sized reaching almost to the anterior margin of sternite VIII; vaginal sclerite complex oviform.

Etymology. We dedicated this species to Professor Wolfram Graf who has collected this species to appreciate his significant contribution to the knowledge of Drusus genus.

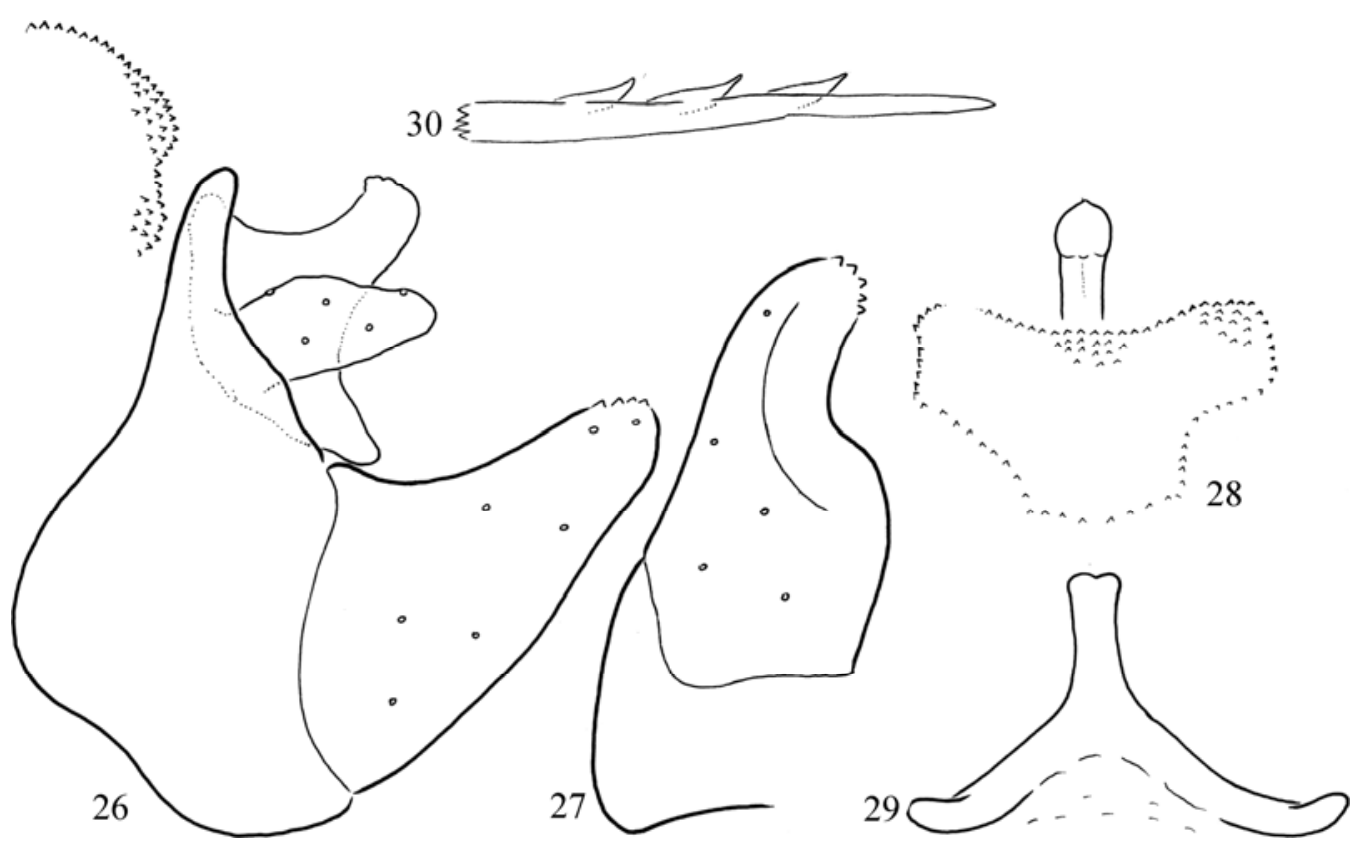

Figures 26-30. Drusus grafi Oláh, sp. nov. $26=$ male genitalia in left lateral view, $27=$ left gonopod in ventral view, 28 = paraproct dorsal branch and spinulose pattern on tergite VIII in dorsal view, $29=$ paraproct dorsal and ventral branches in caudal view, $30=$ apical section of left paramere in lateral view. 


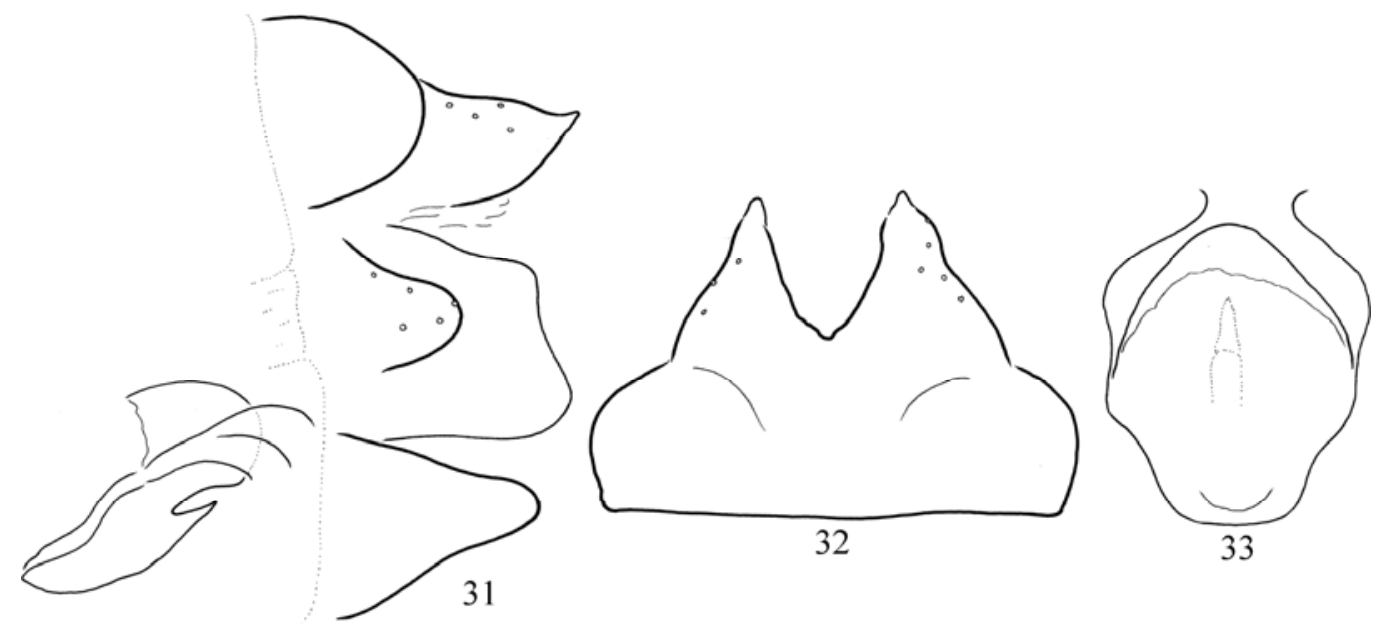

Figures 31-33. Drusus grafi Oláh, sp. nov. 31 = female genitalia with dorsal vaginal sclerite complex in left lateral view, 32 = fused tergite IX and $\mathrm{X}$ in dorsal view, 33 = dorsal vaginal sclerite complex in dorsal view.

\section{Drusus gredosensis Oláh, sp. nov.}

(Figures 34-39, 40-42)

Drusus bolivari (McLachlan, 1876): Schmid 1952a: 680. (Spain, Gredos Mts.). Misidentification.

Drusus bolivari (McLachlan, 1876): Schmid 1956: 51-52. Misidentified male and female specimens from the Gredos Mts. Moreover synonymised D. estrellensis (McLachlan, 1884), Portugal, Estrella Mts, with D. bolivari (McLachlan, 1876), Spain, Guadarrama Mts.

Drusus bolivari (McLachlan, 1876): Malicky 1983: 165. Misidentification.

Diagnosis. Drusus gredosensis sp. nov. differs from D. bolivari (McLachlan) by having lateral aspect of paraproct differently formed; apex pointed and upward as well as slightly backward turning, not straight horizontal and not bifid dorsoapicad. Paraproct has slender, slightly recurving, hook-like apex in lateral view, the most slender in the species complex. Cerci are with elongated apical ventrum. Other periphallic structure, the gonopods differs from all the other related species by having no mesad directed apex in ventral view. Number of paramere spines, the modified setae is very high 11-12. Paramere with 11 modified setae differs from all the other species in the complex; those are with much less setae. In the holotype the modified setae of the paramere are lying recumbent, probably a more virgin condition before copulation. In paratypes the setae are less recumbent, more erect, probably worn during copulation.
Material examined. Schmid (1952a) has collected several Drusus specimens from Spain (Sierra de Gredos) and determined as Drusus bolivari (McLachlan). He has deposited 3 males and 1 female in The Natural History Museum, London. Holotype. Spain (Av.) Lac de Gredos, 16-23. VII. 1950, leg F. Schmid, Brit. Mus. 1951-132, BMNH(E) 1253663 (1 male, NHM). Allotype. Lac de Gredos, 16-23. VII. 1950, leg F. Schmid, Brit. Mus. 1951-132, BMNH(E) 1253665 (1 female, NHM). Paratypes. Spain (Av.) Lac de Gredos, 1623. VII. 1950, leg F. Schmid, Brit. Mus. 1951-132, BMNH(E) 1253664 (1 male, NHM). Spain (Av.) Spain (Av.) Lac de Gredos, 16-23. VII. 1950, leg F. Schmid, Brit. Mus. 1951-132, BMNH(E) 1253666 (1 male, NHM).

Description. Body, appendages and wing colour, nervulation, male and female genitalia are described in detail by Schmid (1956) under the name of $D$. bolivari.

Etymology. We named this species after the mountain range where the type material was collected.

\section{Drusus jesusi Oláh, sp. nov.}

(Figures 43-47, 48-50)

Diagnosis. The single male and female specimens available for this study were collected by Prof. Gonzalez together with male and female specimens of Drusus gonzalezi sp. nov. in the Ancares Mts. The females were simply sizeassociated, need further confirmation. Most close to Drusus bolivari McLachlan described from the Guadarrama Mts. at least by comparing with the 
permanent slide preparate of the single holotype male of $D$. bolivari. The permanent preparate, although of very good quality, permits examination only in lateral view with slightly left caudal distortion. Moreover the paramere pattern is not visible. Further studies are required to give detailed comparative trait analysis of the two species. The type locality of the single Drusus bolivari specimen is far from the type locality of Drusus jesus sp. nov. There are several incipient species inhabiting mountain ranges nearby the type locality of $D$. bolivari in the Guadarrama Mts.: Drusus estrellensis McLachlan in Estrella Mts., D. gredosensis sp. nov. in the Gredos Mts., D. grafi sp. nov. in the Demanda Mts.

Material examined. Holotype. Spain, NW Spain, Sierra de Ancares, Lugo, Rio de la Vara, Rio da Vara, N42 $48^{\prime}$ 10.74", W6 53'19.90”, 1230m, 14.VII.1984, leg. Marcos A. Gonzalez (1 male, OPC). Allotype. Same as holotype (1 female, OPC).

Male genitalia. Apical spinulose area on tergit VIII slightly trilobed with short anterior mesal band. Cerci low with produced ventroapical lobe. Paraproct dorsal branch slender straight, with slightly bifid dorsoapical hook and irregular dorsal margin in lateral view, simple digitate with broader basal part in dorsal view; and more pointed in caudal view; paraproct ventral branch without sclerotized ventrum, widely expanded laterad with mesad turning pointed hooks. Gonopods short robust in lateral view and mesad turning in ventral view. Paramere with long and slender apical rod, shaft terminal forming a pointed broad spine preceded by a thin spine-like seta.

Female genitalia. Tergum IX forms a short tube open ventrally; tergum $\mathrm{X}$ deeply bilobed in dorsal view. Lateral setose lobe, the sternite of segment IX rounded. Supragenital plate of sternum $\mathrm{X}$ well-developed and rounded in lateral view. Median lobe of the vulvar scale (lower vaginal lip) well developed. Vaginal chamber medium sized reaching almost to the anterior margin of sternite VIII, vaginal sclerite complex oviform with narrowing anteriad in dorsal view.

Etymology. We dedicated this species to Dr. Jesús Martínez Menéndez who has paid desperate trials to delimit species in the Drusus bolivari complex with neutral DNA markers.

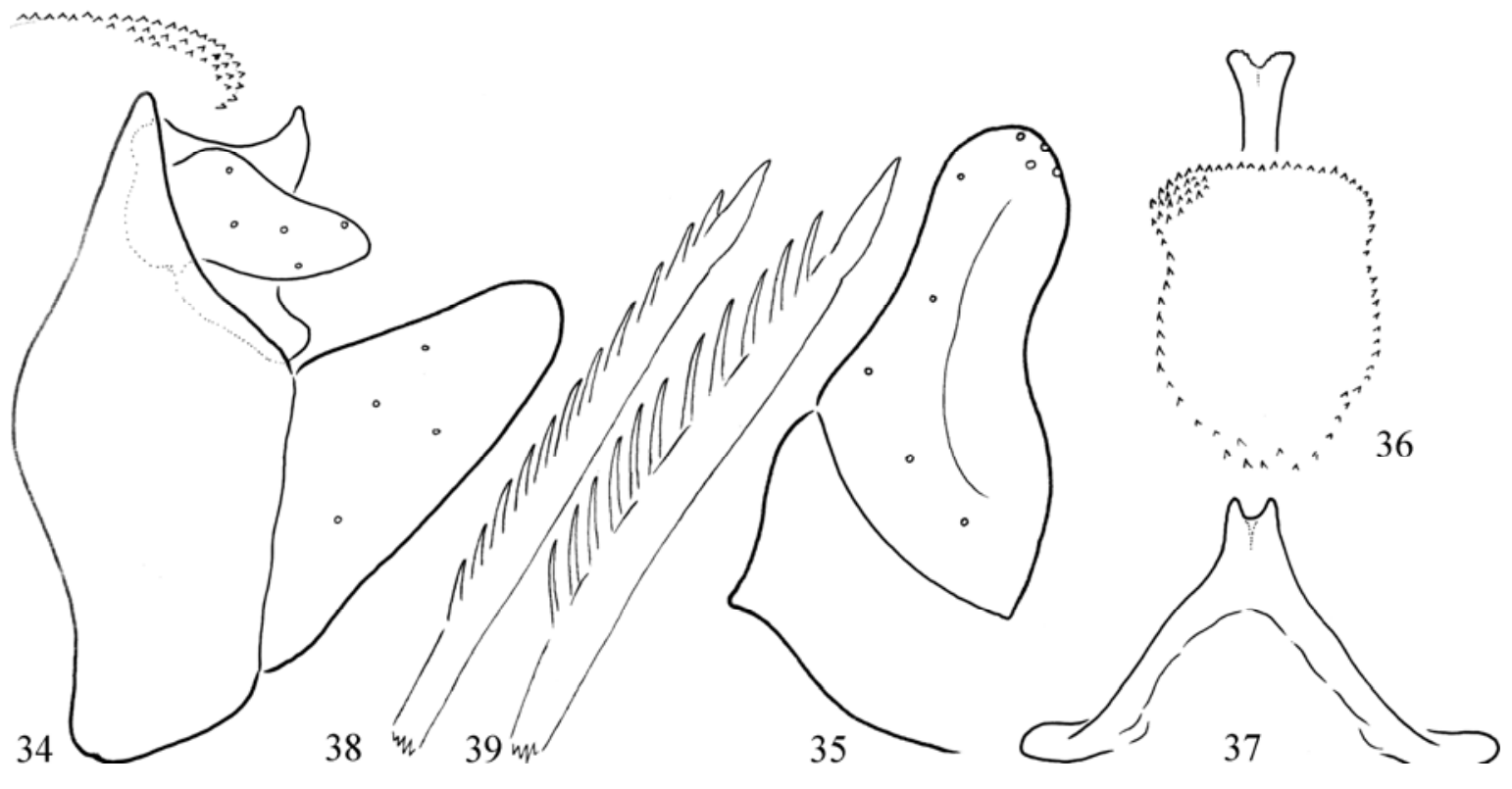

Figures 34-39. Drusus gredosensis Oláh, sp. nov. $34=$ male genitalia in left lateral view, $35=$ left gonopod in ventral view, $36=$ paraproct dorsal branch and spinulose pattern on tergite VIII in dorsal view, 37 = paraproct dorsal and ventral branches in caudal view, 38 = apical section of left paramere of holotype in lateral view, $39=$ apical section of left paramere of one paratype in lateral view. 


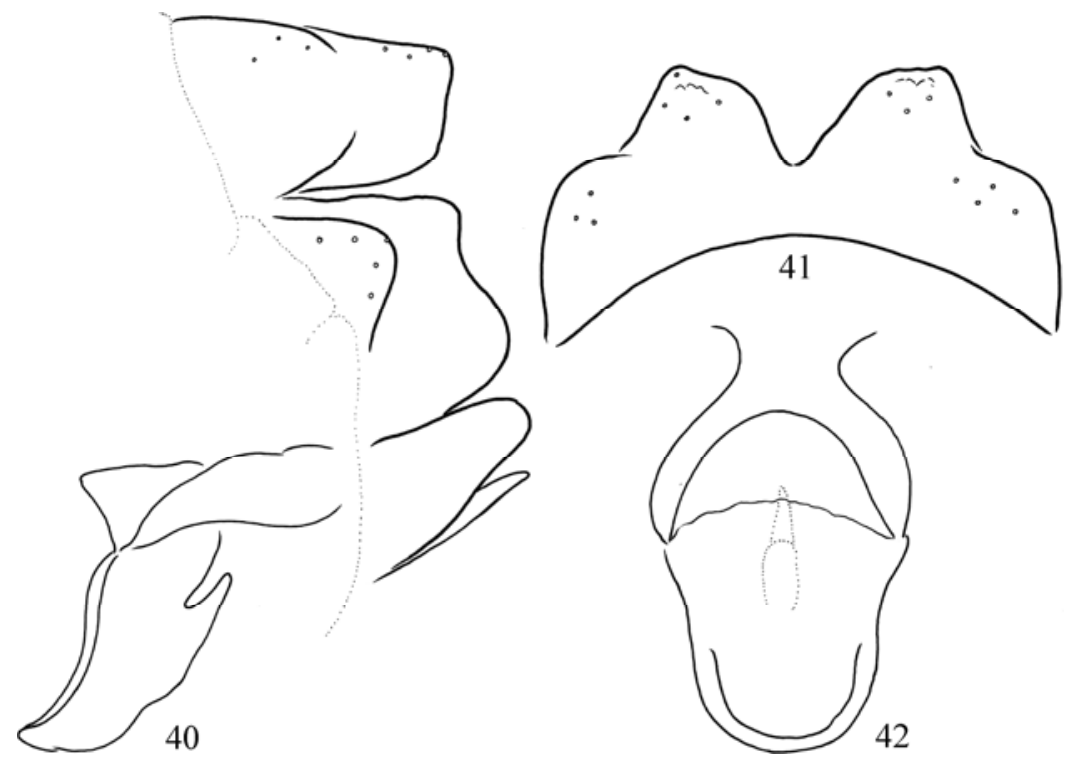

Figures 40-42. Drusus gredosensis Oláh, sp. nov. 40 = female genitalia with dorsal vaginal sclerite complex in left lateral view, 41 = fused tergite IX and X in dorsal view, 42 = dorsal vaginal sclerite complex in dorsal view.
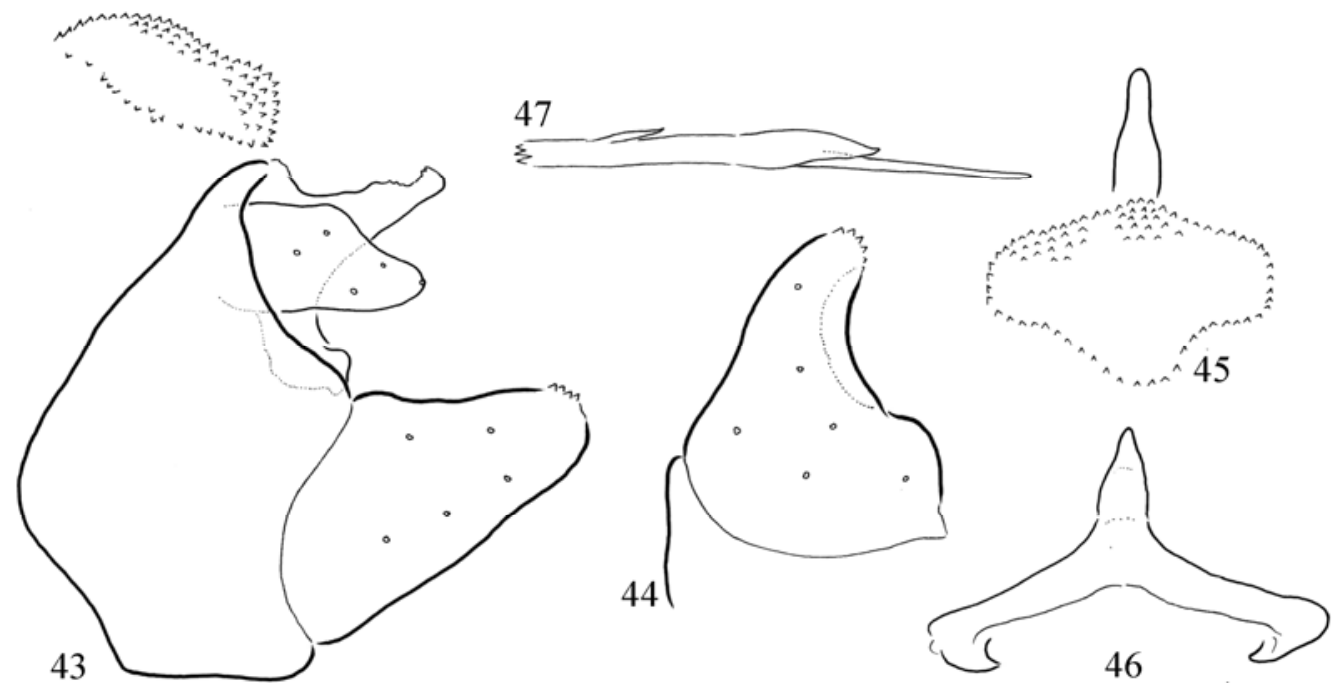

Figures 43-47. Drusus jesusi Oláh, sp. nov. 43 = male genitalia in left lateral view, $44=$ left gonopod in ventral view, $45=$ paraproct dorsal branch and spinulose pattern on tergite VIII in dorsal view, $46=$ paraproct dorsal and ventral branches in caudal view, 47 = apical section of left paramere in lateral view.

\section{Drusus pyrenensis Oláh \& Coppa, sp. nov.}

(Figures 51-55)

Diagnosis. Most close to D. estrellensis (McL.), but differs by having spinulose area on tergit VIII less trilobed; cerci differently shaped, long and low, not short and high; paraproct dorsal branch less robust in lateral view and paraproct ventral branch less expanded; gonopods elongated slender, not short and robust; paramere with 3-5 modified setae, not with 6-7 spine-like setae.

Material examined. Holotype. France, Department Hautes-Pyrénées, Bagnères-de-Bigorre, Soulagnet bord du torrent, source de Labassère, 2. VII. 2013 leg. G. Coppa, (1 male, CPC). Paratypes. Same as holotype (2 males, CPC; 2 males, OPC). 
Description. A new species with brown body and wings. Head and thoracic sclerites darker brown; setal warts, both on the head and thorax, and palps, antennae and legs lighter brown. Spur formula 1,2,3. Forewing length $10 \mathrm{~mm}$.

Male genitalia. Apical spinulose area on tergit VIII less distinctly trilobed. Cerci low and long quadrangular. Paraproct dorsal branch slender with obliquely serrated dorsoapical margin in late- ral view, simple digitate in dorsal view; paraproct ventral branch without sclerotized ventrum, widely expanded laterad. Gonopods elongated slender in lateral view and mesad turning in ventral view. Parameres with 3 to 5 modified setae.

Etymology. We named this species after the mountain range where the type material was collected.

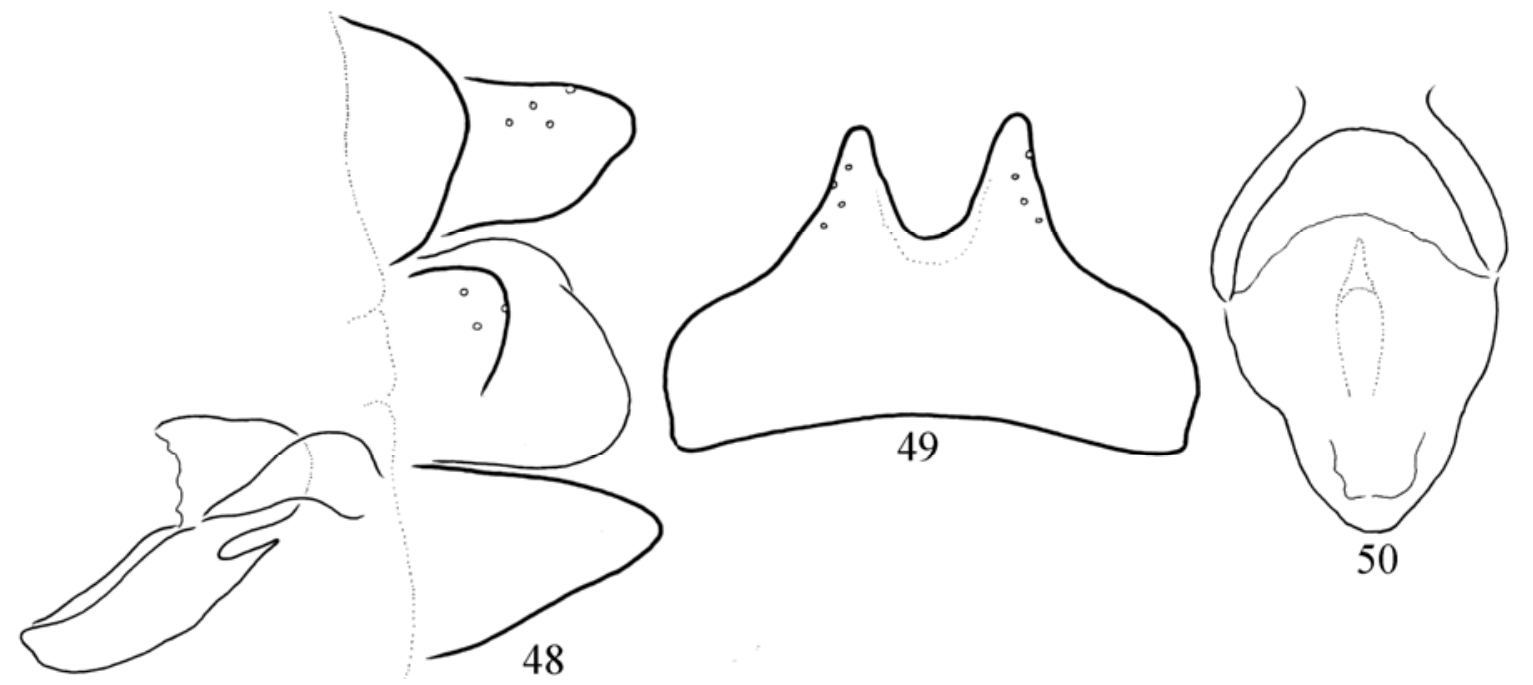

Figures 48-50. Drusus jesusi Oláh, sp. nov. $48=$ female genitalia with dorsal vaginal sclerite complex in left lateral view, 49 = fused tergite IX and $\mathrm{X}$ in dorsal view, 50 = dorsal vaginal sclerite complex in dorsal view.
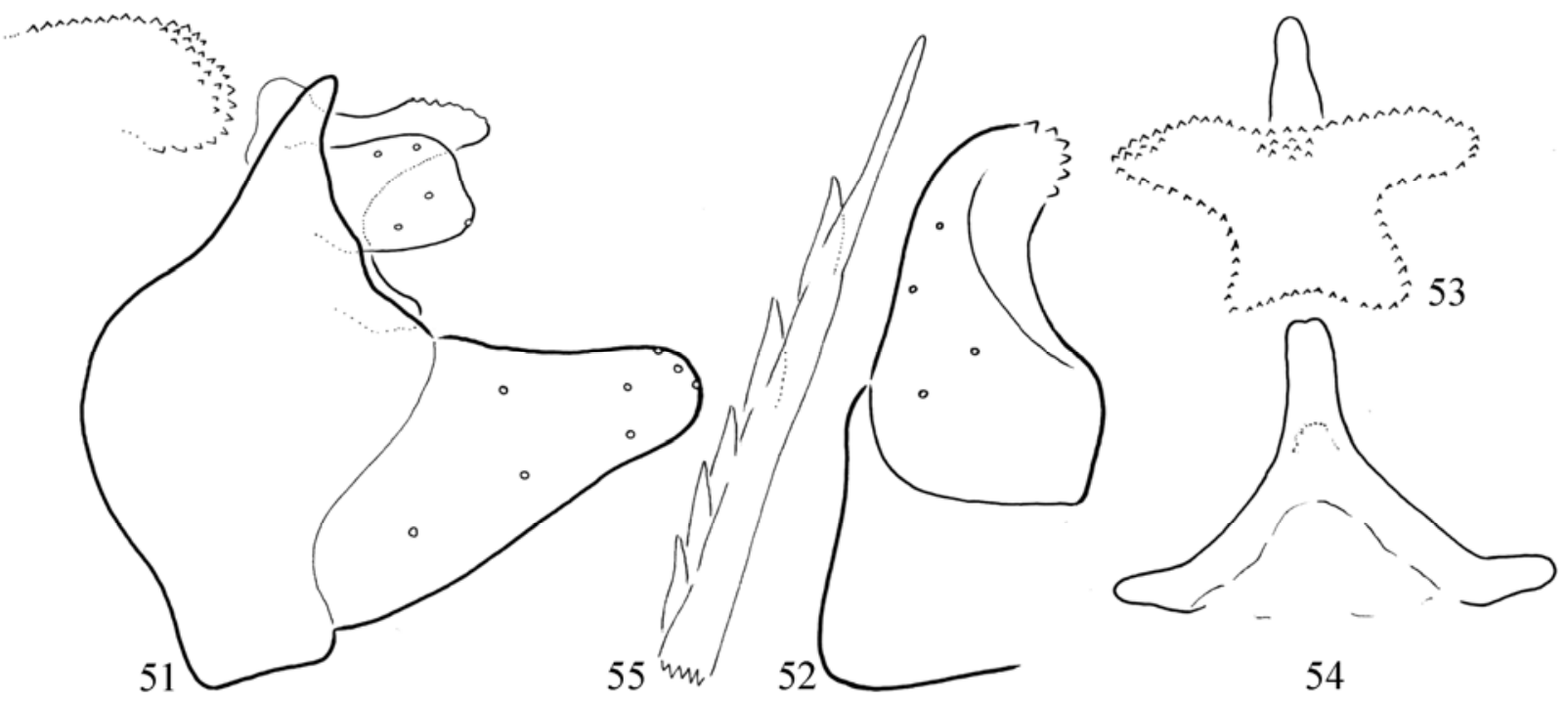

54

Figures 51-55. Drusus pyrenensis Oláh \& Coppa, sp. nov. $51=$ male genitalia in left lateral view, $52=$ left gonopod in ventral view, 53 = paraproct dorsal branch and spinulose pattern on tergite VIII in dorsal view, 54 = paraproct dorsal and ventral branches in caudal view, 55 = apical section of left paramere in lateral view. 
Subfamily Limnephilinae Kolenati, 1859

Tribe Stenophilacini Schmid, 1955

\section{Genus Isogamus Schmid, 1955}

Isogamus Schmid, 1955: 183-184. Type species: Anisogamus aequalis Klapálek, 1907

This small endemic Carpathian genus inhabits spring-fed streams in the alpine, subalpine, and montane zones of the East and South Carpathians. The genus is highly heterogeneous comprising of the isolated, highly specialised apomorphic Isogamus lineatus (Klapálek, 1901) species and the contemporary evolved Isogamus aequalis species complex.

Isogamus lineatus forms possibly an independent genus having completely different genital architecture, gross structure and fine structure, representing both neutral and non-neutral adaptive traits, probably evolved in different divergence ages or splitting times. At this time we have no any theoretical model to estimate phylogenetic age for architectural gross and fine structural divergences. A more sophisticated model for elaboration of trait divergence ages and a comprehensive comparative examination of relevant genera are required to revise the Stenophylacini tribe.

In the I. aegualis species complex the speciation processes, detected by speciation trait of the paramere and of the paraproct, produced 2 known and 2 new incipient species described here. More unknown incipient species could be discovered in the poorly known isolated ranges of the East Carpathians. We have collected a single unknown female specimen in the Gurghiu Mts. near Bucin Pass. Isogamus aequalis and I. czarnohorensis described from the North-East Carpathians are close relatives, forming a sibling pair of incipient species as compared to the newly described sibling pair of I. balinti sp. nov. and I. baloghi sp. nov.

The revision of this small genus is based on newly collected materials and on the elaboration of the historical material of Klapálek's Collection deposited in NMPC (Czech Republic, Prague).
Isogamus aequalis new species complex is characterized by very high and very short segment IX; vertically elongated gonopods with blunt apex; its articulation to the apical margin of segment IX is flexible at least on its dorsal region; permitting some free movement which produces various distances between the gonopod anterior margin and the superanal genitalic complex at the different specimens,. Lateral shoulder of segment IX very short. The superanal genitalic complex is almost entirely framed ventrally by the mesad directed digitate midlateral sclerotized strips.

Isogamus aequalis-czarnohorensis new sibling pair is characterized by having blunt gonopod apex in lateral view; dorsally concave paraproct, curving upward; parameres with more developed spine pattern of the modified setae; paramere shaft terminal robust spine-like at I aegualis or reduced almost to the size of the accompanied modified setae at I. czarnohorensis.

Isogamus balinti-baloghi new sibling pair is characterized by having truncate, quadratic or even concave gonopod apex in lateral view; dorsally straight or convex paraproct; parameres with less developed spine pattern of the modified setae; paramere shaft terminal slender spine-like.

\section{Isogamus aequalis (Klapálek, 1907)}

(Figures 56-65, 66-82)

Anisogamus aequalis Klapálek, 1907: 24-27.

Isogamus aequalis (Klapálek, 1907): Transferred to the newly erected genus Isogamus g. n. by Schmid 1955: $183-184$.

Isogamus aequalis (Klapálek, 1907): Differential diagnoses of both sexes of I. aequalis and I. czarnohorensis from the Ukrainian Carpathians were elaborated and detailed drawings prepared by Szczęsny 1980: 466-471.

Isogamus aequalis (Klapálek, 1907): The first collection and reliably records from Slovakia with excellent drawings was presented by Chvojka 1993: 217-220.

Material examined. Poland, Bieszczady Mts. X. leg. B. Szczęsny (2 males, 2 females; OPC). Slovakia, Vihorlat Mts. Zemplinske Hamre, 700 m, 15. IX. 1962, leg. J. Sýkora (3 males, 4 females, aedeagus and parameres on slide No. 13,14, NMPC; 3 males, 2 females, OPC). Vihorlat Mts. source area, brooklet nr. Morske oko, 15.IX.1962, leg. J. Sýkora, (4 males, aedeagus and parameres on slide No. 16, NMPC). Vihorlat, 16. IX. 1962, leg. J. Sýkora (2 males, ae- 
deagus and parameres on slide No. 1,2, NMPC). Vihorlat Mts. source area above Zemplinske Hamre, $740 \mathrm{~m}, 12 . \mathrm{X}$. 1990, leg. P. Chvojka (1 male, aedeagus and parameres on slide No. 18, NMPC). Vihorlat Mts. source area above Zemplinske Hamre, 740 m, 12.X.1990, leg. P. Chvojka (1 male, aedeagus and parameres on slide No. 18, NMPC). Vihorlat Mts. Bukovské vrchy Hills, brooklet north of Nová Sedlica, 10.X.1990, leg. P. Chvojka (1 male, aedeagus and parameres on slide No. 17, NMPC). Vihorlat Mts. left tributary of Bystrá stream, 5-700 m, 9.X.1990, leg. P. Chvojka (4 males, aedeagus and parameres on slide No. 16, NMPC). Ukraine, Klapálek Collection: No. 21, Worochtensky, 7.IX.1908, leg. J. Dziędzielewicz (1 female, NMPC). Klapálek Collection: No. 5, Chomiak (Błotek), 22.IX.1906, leg. J. Dziędzielewicz (1 male, aedeagus and parameres on slide No K5, NMPC). No. 6, Chomiak, 5.IX.1908, leg. J. Dziędzielewicz (1 male, aedeagus and parameres on slide No.
K6, NMPC). No. 7, Chomiak, 5.IX.1908, leg. J. Dziędzielewicz (1 male, aedeagus and parameres on slide No. K7, NMPC). No. 8, Chomiak, 5.IX.1908, leg. J. Dziędzielewicz (1 male, aedeagus and parameres on slide No K8, NMPC). No. 9, Chomiak, 5.IX.1908, leg. J. Dziędzielewicz (1 male, aedeagus and parameres on slide No K9, NMPC). No. 10, Chomiak, 5.IX.1908, leg. J. Dziędzielewicz (1 male, aedeagus and parameres on slide No K10, NMPC). No. 20, Chomiak (Błotek), 22.IX.1906, leg. J. Dziędzielewicz (1 female, NMPC). Bieszczady Mts (Besszádok), Ung National Park, below Lubnya (Kiesvölgy), N: $49^{\circ} 00^{\prime} 54,81^{\prime \prime}$ E: $22^{\circ} 43^{\prime}$ 23,82”, 478 m, singled, 20. IX. 2013, leg. J. Oláh, Cs. Balogh, Cs. Deák \& I. Meszesán (1 female, OPC).

Remarks. Earlier records from Romania (Botosaneanu 1961) are misidentifications.

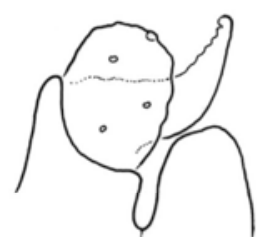

56

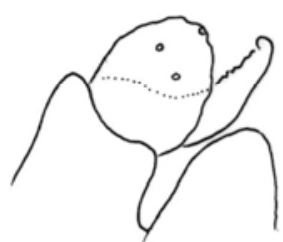

61

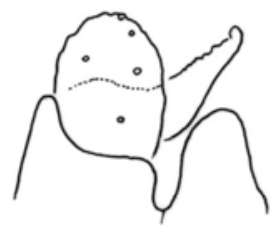

57

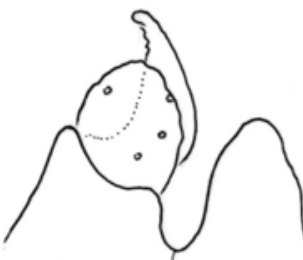

62

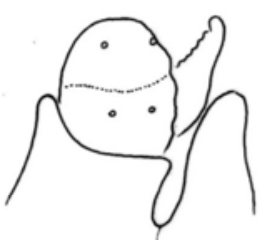

58

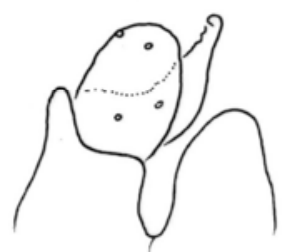

63

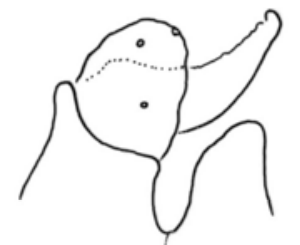

59

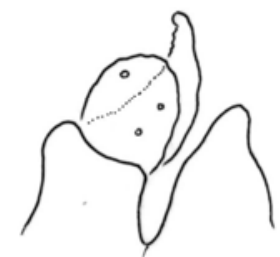

64

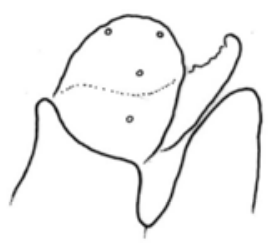

60

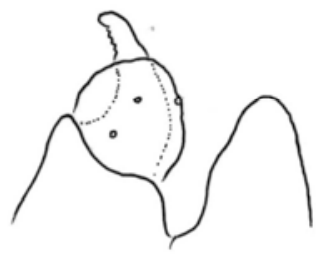

65

Figures 56-65. Isogamus aequalis (Klapálek, 1907). 56-60 = superanal genitalic complex with tergite IX and gonopods tip from Chomiak Mts. Ukraine, 61-63 = superanal genitalic complex with tergite IX and gonopods tip from Vihorlat Mts. Slovakia, 64-65 = superanal genitalic complex with tergite IX and gonopods tip from Bieszczady Mts. Poland.

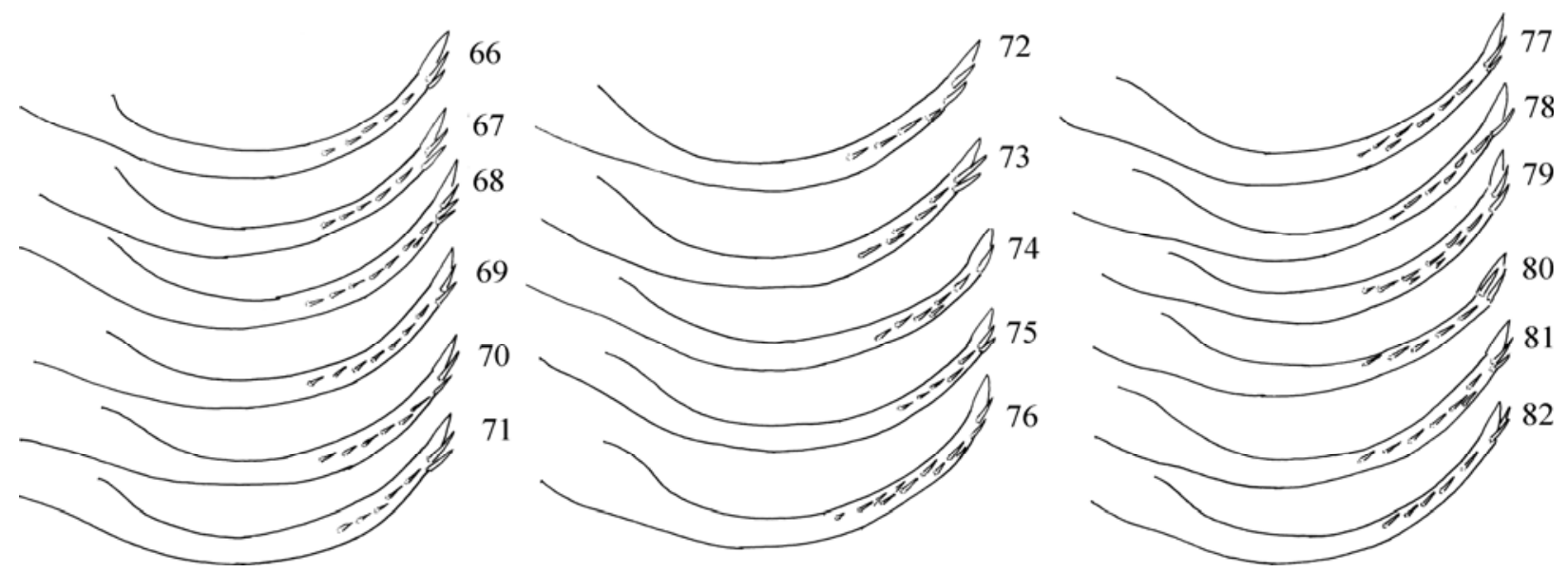

Figures 66-82. Isogamus aequalis (Klapálek, 1907). 66-71 = left paramer in lateral view from Chomiak Mts. Ukraine, $72-73=1$ eft paramer in lateral view from Bieszzcady Mts. Poland. 74-82 = left paramer in lateral view from Vihorlat Mts. Slovakia. 


\section{Isogamus balinti Oláh, sp. nov.}

(Figures 83-90)

Isogamus aequalis aequalis Klapálek, 1907. Records from South Carpathians by Botosaneanu 1961: 60. Misidentification.

Diagnosis. Sibling species of $I$. baloghi having truncate, concave gonopod apex in lateral view; dorsally convex paraproct; parameres with less developed spine pattern of the modified setae; shaft terminal slender spine-like. Differs from its sibling $I$. baloghi by having high paraproct in lateral view, not slender, paramere dorsum convex, not straight; reduced spine-like setae located ventrad, not laterad. Female unknown.

Material examined. Holotype. Romania, Southern Carpa- thians, Parâng Mts. Calcescu Lake, 45.356 23.614, 1802 m, 3.VIII.2004, leg. L. Ujvárosi (1 male, OPC). Paratypes. Same as holotype (1 male, OPC). Southtern Carpathians, Ieser Mts, 45.45 25.02, 1050m, 3.VIII.2006, leg. M. Bálint (2 males, OPC).

Male genitalia. Apical spinulose area on tergit VIII less developed, slightly bilobed. Cerci rounded. Paraproct convex dorsad, with serrated dorsum. Gonopods vertically elongetad with concave apex. Paramere shaft with slender terminal spinelike shape accompanied by reduced spine-like modified setae located ventrad.

Etymology. We dedicated this species to Miklós Bálint, one of the collectors who have accompanied the senior author (J.O.) in his first Romanian Carpathian collecting trip.
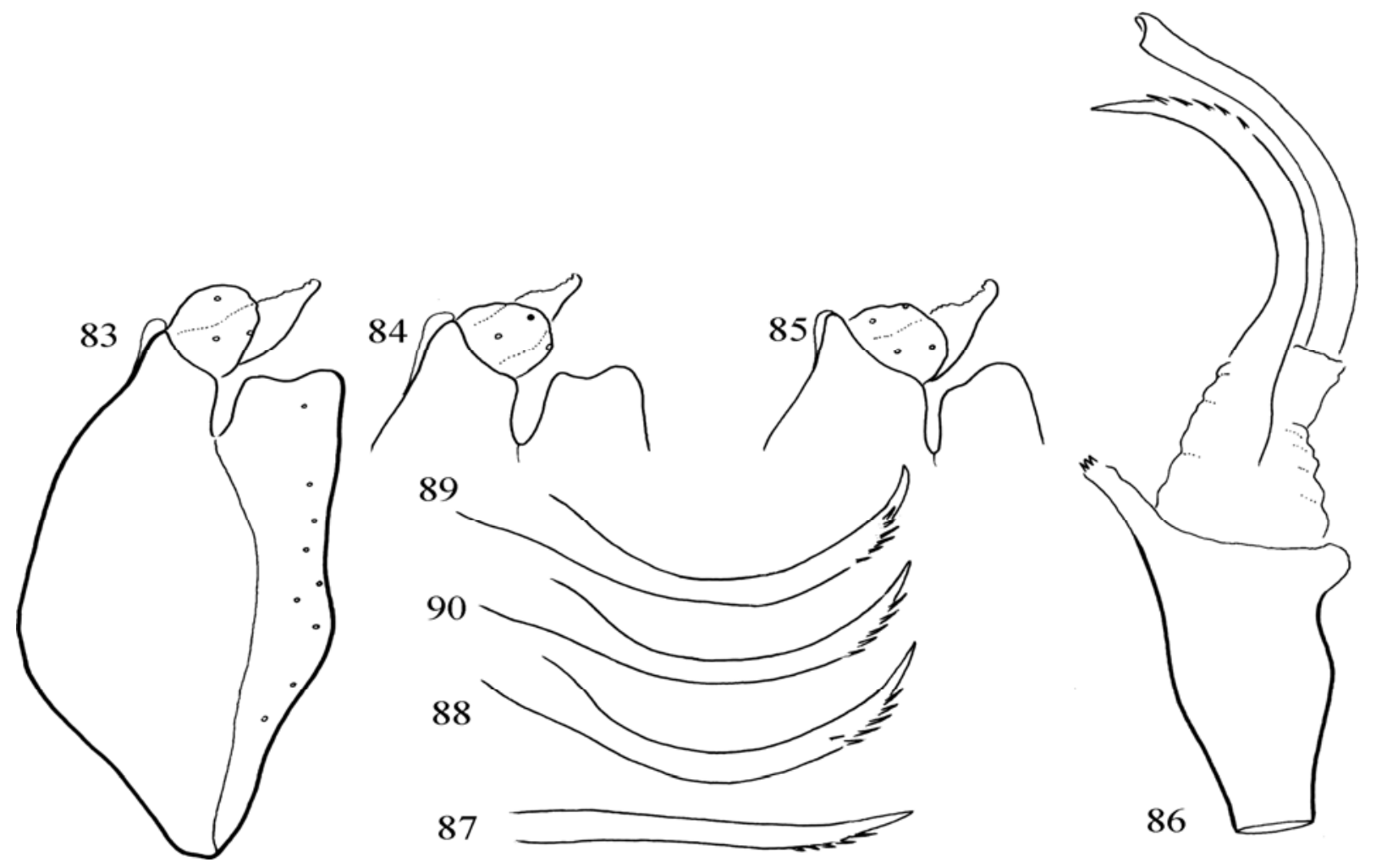

Figures 83-90. Isogamus balinti Oláh \& Chvojka, sp. nov. $83=$ holotype male genitalia in left lateral view, $84=$ superanal genitalic complex with tergite IX and gonopods tip of holotype from Parang Mts. Romania, $85=$ superanal genitalic complex with tergite IX and gonopods tip of paratype from Ieser Mts. Romania, 86 = phallic organ of holotype in lateral view. $87=$ left paramere of holotype in dorsal view, $88=$ left paramere of paratype from the Parang Mts. in lateral view, 89-90= left paramere of paratype from the Ieser Mts. in lateral view. 

tions. at female of $I$. aequalis. $\&$ Cs. Balogh (1 male, OPC).

Isogamus baloghi Oláh, sp. nov.

(Figures 91-93, 94-101, 102-110, 111-113)

Isogamus aequalis aequalis Klapálek, 1907. Records from Rodnai Mts. by Botosaneanu 1961: 60. Misidentifica-

Diagnosis. Sibling species of I. balinti having truncate, quadratic gonopod apex in lateral view; dorsally straight paraproct; parameres with less developed spine pattern of the modified setae; paramere shaft terminal slender spine-like. Differs from its sibling I. balinti by having slender paraproct in lateral view, not broad, paramere dorsum straight, not convex; reduced spine-like setae on the paramere located laterad, not ventrad. Female lateral lobes of tergite IX long triangular not short and not blunt like at I aequalis; basal region of tergite IX shouldered and without subbasal dark crossline, not without lateral shoulder and not with pronounced subbasal transversal line present

Material examined. Holotype. Romania. Rodnei Mts. Iza stream, side spring with sphagnum bog, N47 $36^{\prime} 19.3^{\prime \prime}$ E2431'53.4", 993m 27. IX. 2014, leg. J. Oláh \& Cs. Balogh (1 male, OPC). Allotype. Same as holotype (1 female). Paratype. Same as holotype (10 males, 2 females, OPC; 4 males, 1 female, CSNMB). Rodnei Mts. Numerous spring streamlets on the spring area of Cailor waterfall, Piatra Rea, N47 35'1.9” E2447’49.4”, 1564m, 28. IX. 2014, leg. J. Oláh

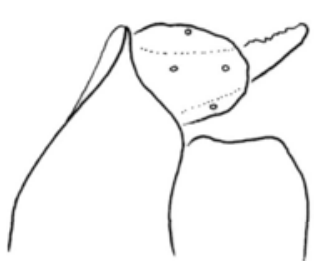

94

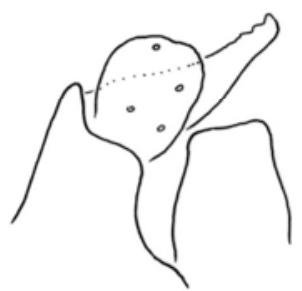

98

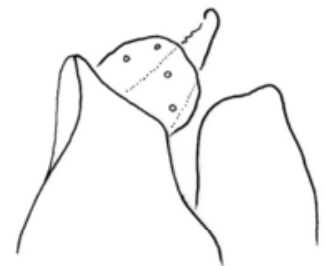

95

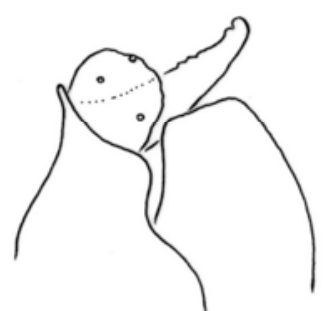

99
Male genitalia. Apical spinulose area on tergit VIII less developed, slightly bilobed. Cerci rounded. Paraproct straight, with serrated dorsum subapicad. Gonopods vertically elongetad with truncate apex. Paramere shaft with slender terminal spine-like shape accompanied by reduced spinelike modified setae located laterad, except two or three located ventrad, subapicad.

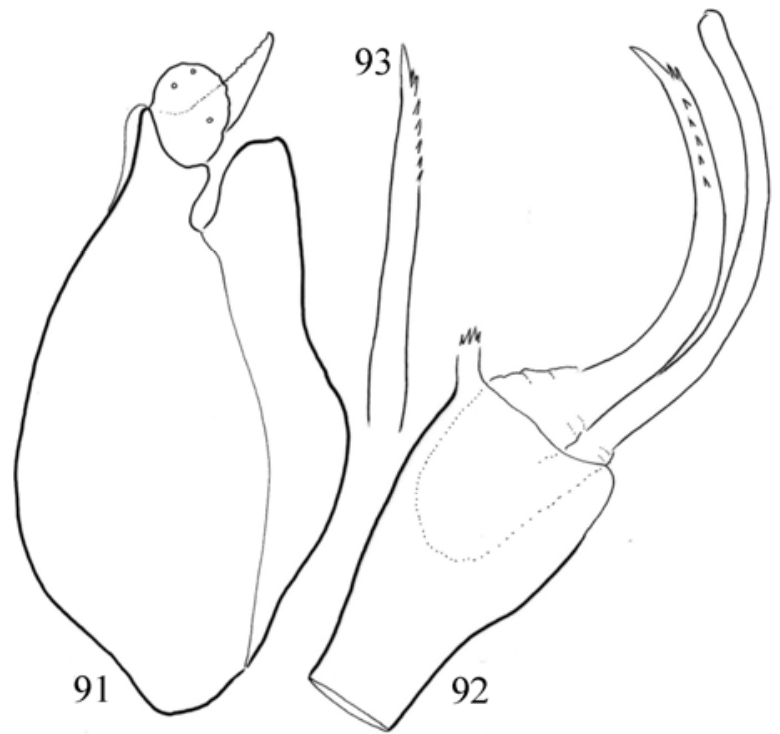

Figures 91-93. Isogamus baloghi Oláh \& Chvojka, sp. nov. $91=$ holotype male genitalia in left lateral view, $92=$ phallic organ of holotype in lateral view, $93=$ left paramere of holotype in dorsal view.

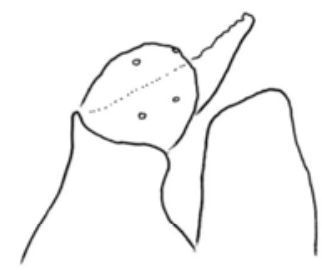

96

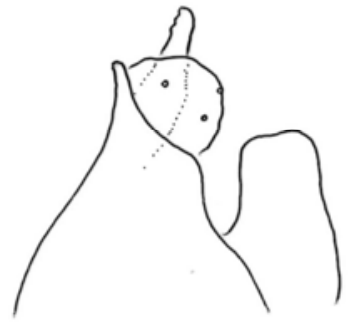

100

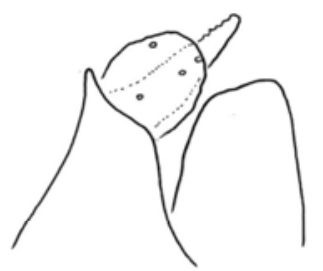

97
Figures 94-101. Isogamus baloghi Oláh \& Chvojka, sp. nov. Superanal genitalic complex with tergite IX and gonopods tip of paratypes. 
Female genitalia. Tergum IX bilobed in dorsal view with deep V-shaped excision; basal region broader producing lateral shoulders. Tergum $\mathrm{X}$ less sclerotized and slightly protruding and excised mesad as visible both in dorsal and ventral view. Lateral setose lobe, the sternite of segment IX fused with the supragenital plate of sternum $\mathrm{X}$ and dominating on female genitalia. Median lobe of the vulvar scale (lower vaginal lip) small and rounded. Vaginal sclerite complex short with abbreviated spermathecal process.

Etymology. We dedicated this species to Csaba Balogh, one of the collectors who has accompanied the senior author (J.O.) in several Carpathian collecting trips.

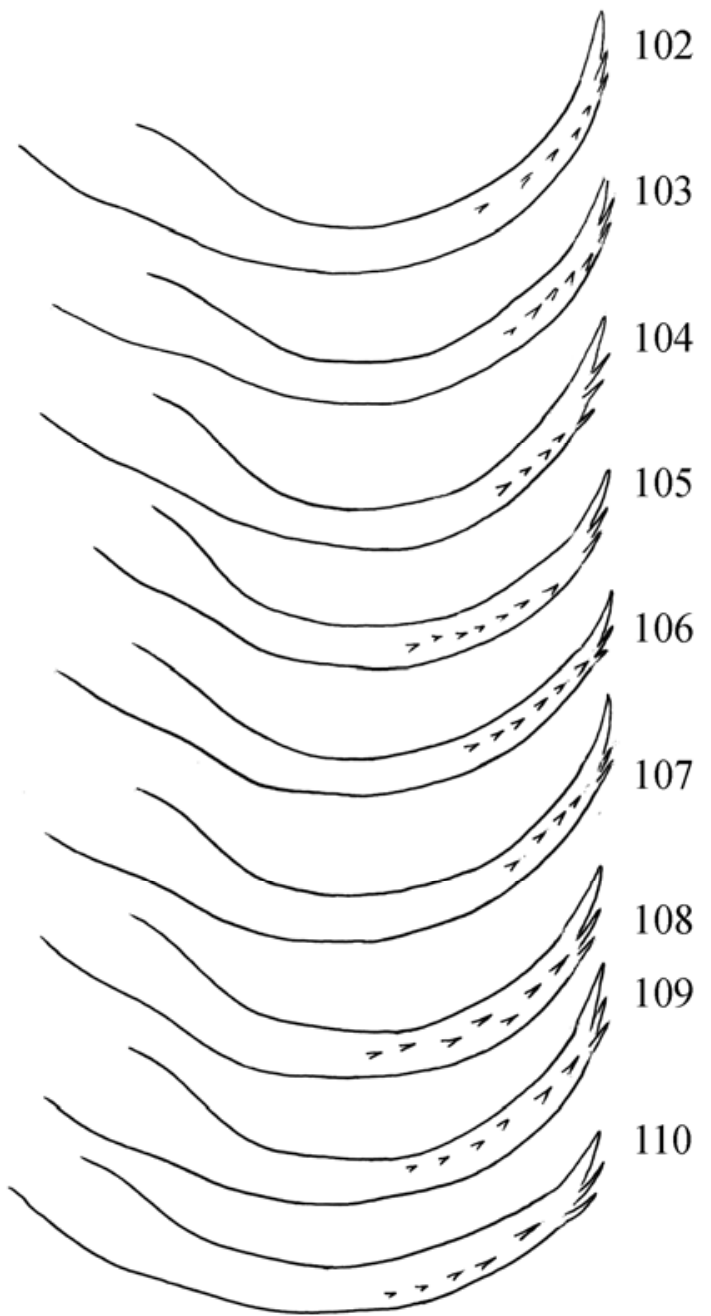

Figures 102-110. Isogamus baloghi Oláh \& Chvojka, sp. nov. Left paramere of paratypes in lateral view.

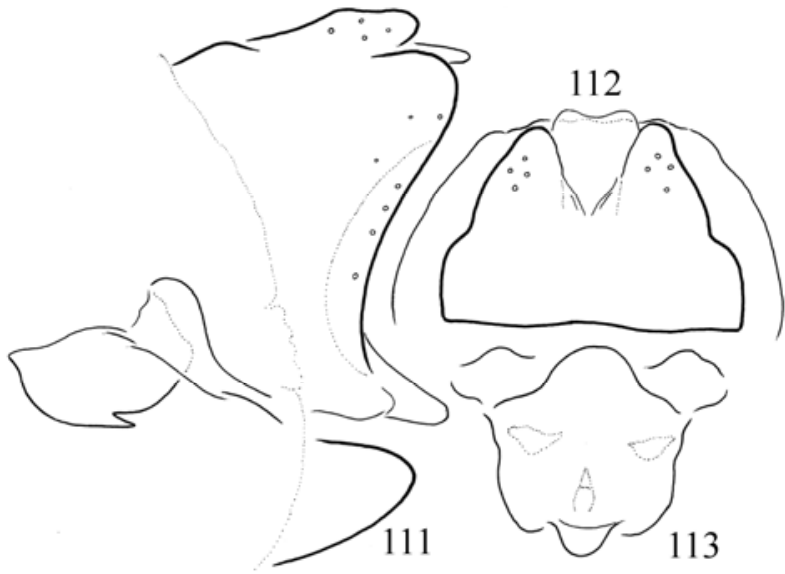

Figures 111-113. Isogamus baloghi Oláh \& Chvojka sp. nov. $111=$ female genitalia with dorsal vaginal sclerite complex in left lateral view, 112 = fused tergite IX and $X$ in dorsal view, $113=$ dorsal vaginal sclerite complex in dorsal view.

\section{Isogamus czarnohorensis (Dziędzielewicz, 1912)}

(Figures 114-120, 121-129)

Anisogamus aequalis Klapálek, var. czarnohorensis Dziędzielewicz, 1912: 137-138.

Isogamus aequalis czarnohorensis (Dziędzielewicz, 1912): Transferred to the newly erected genus Isogamus g. n. by Schmid 1955: 183-184.

Isogamus czarnohorensis (Dziedzielewicz, 1912): stat. nov. Elevated to species rank by Szczęsny 1980: 467-469. Lectotype and paralectotypes designated by Szczęsny 1980: 467-169.

Isogamus czarnohorensis (Dziędzielewicz, 1912): Differential dianoses of both sexes of I. aequalis and I. Czarnohorensis from the Ukrainian Carpathians were elaborated and detailed drawings prepared by Szczęsny 1980: 466471.

Material examined. Romania. Maramures county, Maramaros Mts. Hututeanca stream, $1020 \mathrm{~m}, \mathrm{~N} 47^{\circ}$ 52' $27^{\prime}$ ' E24 20' 31', 7.VIII.2012, leg. J. Oláh \& L. Szél (1 male, OPC). Maramures Mts. Bistra stream valley, small spring with sphagnum bog, N47 53'1.3" E24 ${ }^{\circ} 16^{\prime} 47.7 ”, 609 \mathrm{~m}, 27$. IX. 2014, leg. J. Oláh \& Cs. Balogh (1 male, OPC). Ukraine. Klapálek Collection: K218, Foreszczynka, 12.IX.1908, leg. Dziędzielewicz (1 male, NMPC). K219, Porzyszewska, 9.IX. 1908, leg. Dziędzielewicz (1 male, NMPC). K220, Pod Turkul, 10.IX.1908, leg. Dziędzielewicz (1 male, NMPC). No. 22, Pod Turkul, 10.IX.1908, leg. Dziędzielewicz (1 female, NMPC). No. 23, Pod Breskul, 11.IX.1908, leg. Dziędzielewicz (1 female, NMPC). No. 24, Pod Dancerz, 10.IX.1908, leg. Dziędzielewicz (1 female, NMPC). No. 12, Pod Turkul, 10.IX.1908, leg. Dziędzielewicz (1 male, aedeagus and parameres on slide No K12, NMPC). No. 11, Pod Turkul, 10.IX.1908, leg. Dziędzielewicz (1 male, aedeagus and parameres on slide No K11, NMPC). 


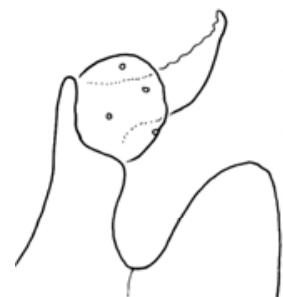

114

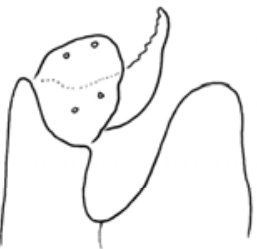

115

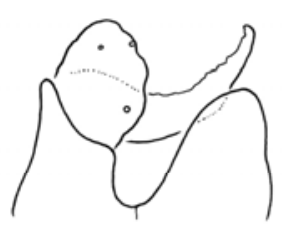

115

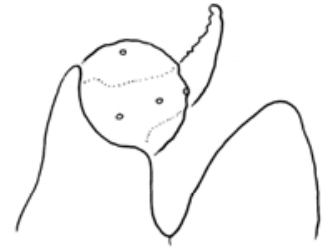

117

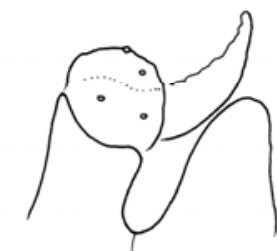

118

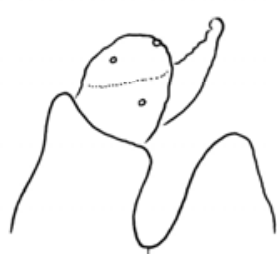

119

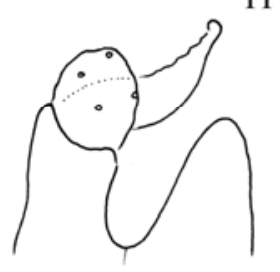

120

Figures 114-120. Isogamus czarnohorensis (Dziedzielewicz, 1912). 114-116= superanal genitalic complex with tergite IX and gonopods tip from Pod Turkul, Ukraine, $117=$ superanal genitalic complex with tergite IX and gonopods tip from Forestenka,

Ukraine, 118 = superanal genitalic complex with tergite IX and gonopods tip from Porzyszewska, Ukraine,

119-120 = superanal genitalic complex with tergite IX and gonopods tip from Marmaros Mts, Romania.

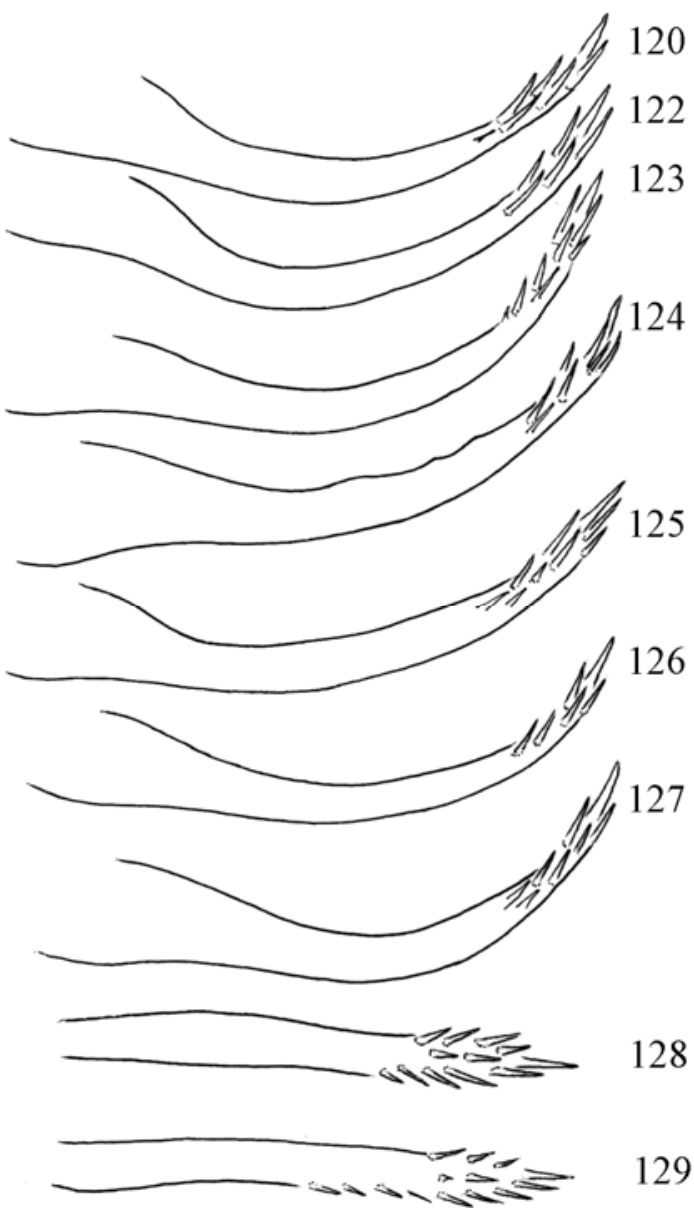

Figures 121-129. Isogamus czarnohorensis (Dziedzielewicz, 1912). $121-123=$ left paramere in lateral view from Pod Turkul, Ukraine, 124 = left paramere in lateral view from Forestenka, Ukraine, $125=$ left paramere in lateral view from Porzyszewska, Ukraine, $126-127=$ left paramere in lateral view from Marmaros Mts, Romania, 128-129 = Left paramere in dorsal view from Marmaros Mts, Romania.

Remarks. According to the original description (Dziędzielewicz 1912) and confirmed by Raciecka (1934) and Szczęsny (1980) this species differs from the closely related sibling species of I. aequalis by its darker colour. We have collected specimens in Romania in sunny environment and the shining dark coloration of the freshly collected living specimens was remarkable.

\section{Isogamus lineatus (Klapálek, 1901)}

Anisogamus lineatus Klapálek, 1901: 1-2.

Isogamus lineatus (Klapálek, 1901): transfered to the genus Isogamus by Botosaneanu 1967: 99, 103.

Material examined. Romania. Argeş county, Făgăraş Mts, Căpătânenii Ungureni, small spring-lake by the Capra Stream along road No.7C, N4534.605' E2437.060', 1405m, 29.VIII.2012 leg. T. Kovács, D. Murányi, J. Oláh (2 males, 5 females; OPC). Argeş county, Făgăraş Mts, Căpătânenii Ungureni, sidebrook of Capra Stream along road No.7C, N45³5.185' E24³7.691', 1705m, 29.VIII.2012 leg. T. Kovács, D. Murányi, J. Oláh ( 8 males, 7 females, 1 copula; OPC). Sibiu county, Făgăraş Mts, Cârțişoara, forest 
seep along road No.7C, N45³8.742' E2436.464', 1325m, 29.VIII.2012 leg. T. Kovács, D. Murányi, J. Oláh (3 females, OPC). Braşov county, Făgăraş Mts, Dejani, forest sidebrook of Dejani stream, N45 $36.408^{\prime}$ E24 56.466 ', 1310m, 30.VIII.2012 leg. T. Kovács, D. Murányi, J. Oláh (8 males, 5 females, OPC). Braşov county, Făgăraş Mts, Dejani, right sidebrook of Dejani stream, N45 $35.446^{\prime}$ E24 $56.348^{\prime}$, 1755m, 30.VIII.2012 leg. T. Kovács, D. Murányi, J. Oláh (1 male, 1 female, OPC). Braşov county, Făgăraş Mts, Dejani, forest sidestream of Dejani stream, N45 $36.720^{\prime}$ E24 56.533', 1250m, 30.VIII.2012 leg. T. Kovács, D. Murányi, J. Oláh (5 males, 3 females, CSNMB).

Remarks. This species was described from Austrian Alp (Styria), but never collected there, despite systematic search, but found very common in the South Carpathians. This confusion was probably resulted by mistakes in the original labelling (Malicky 2005).

\section{Isogamus sp.}

Material examined. Romania. Gurghiu Mts. near Bucin Pass, Tárnava Mica springs and stream, N46 39'16,63", E2516’42,46”, 1290, 30.X.2014, leg. Z. Baczó, Cs. Balogh, J. Kecskés \& J. Oláh. (1 female; OPC)

\section{Genus Melampophylax Schmid, 1955}

Type species: Halesus melampus McLachlan, 1876.

The dark-coloured autumn flying Melampophylax genus has been erected by Schmid (1955), splitting from the genus Halesus. Members of this small genus inhabit subalpine and alpine spring streams and the epirthitron of small streams in Central and West Europe, including Great-Britain. Diverged from Potamophylax-Allogamus-Consorophylax group and most close to Allogamus, but isolated by the formation of male genitalia and by the female vaginal sclerite complex. The unique basal plate elongation on the vaginal sclerite complex forms an anterad directed long tonguelike shape with two heavily sclerotized internal tubes to receive the two unfused parameres. The long rod-shaped parameres armed with specifically shaped terminal blade, knife-shaped as a modified lancing device to help penetration of the parameres into the internal tubes. In Allogamus the elongation of the common duct of the accessory glands with a single internal tube, serves si- milar function to receive the fused paramere (Oláh et al. 2014).

Here we survey this small genus with the speciation trait examining newly collected and historical materials of the Klapálek Collection deposited in NMPC (Czech Republic, Prague), as well as of the Dziędzielewicz's collections deposited both in NHM-ISEA Poland, Krakow) and in SMNHL (Ukraine, Lviv).

This revision focuses on the speciation traits of the parameres, on the apomorphic ventromesal processes of the gonopods evolved in the Melampophylax nepos species complex, as well as on the female vaginal sclerite complex.

Melampophylax genus is composed of two groups: M. melampus group and $M$. mucoreus group. Malicky (1990) has emphasized long ago that the two groups differ much and a revision is required. Yes, all the stenophylacini genera erected by Schmid (1955) are based on genital divergences and the genital architectures of the two groups are basically different. Divergence age or splitting depth in time can be estimated by architecture, gross morphology, and fine structure of both the neutral and the non-neutral adaptive genitalic traits. An elaboration of trait divergence age model of shape analysis is required, applying the theoretical model experiences of molecular genetics, for a comprehensive comparative examination of relevant genera in Stenophylacini.

\section{Melampophylax melampus species group}

Segment IX and gonopods fused; gonopods separated, without ventromesal plate; paraproct composed of several spine-like processes; position of cerci rather transversal, aedeagus with spine-shaped processes, parameres with several spine-like modified setae. Vaginal dorsal sclerite complex not elongated, but accompanied with a unique ventral vaginal sclerite giving ventral support to the membranous and flexible vaginal chamber. The highly specialized male and female genitalia suggest and confirm an earlier divergence of this small group supporting an independent generic taxonomic rank. 


\section{Melampophylax melampus (McLachlan, 1876)}

(Figures 130-131)

Halesus melampus McLachlan, 1876: 158-159.

Material examined. Italy. Lombardia, Mezzoldo, (BG), fiume Brembo, $46,0236^{\circ} \mathrm{N} 9,6611^{\circ} \mathrm{E} 1100 \mathrm{~m}, 3 . X .1995$ leg. F. Albrici, M. Valle (47 males, 12 females, CNSMB; 10 males, 3 females, OPC). Lombardia, Parzanica, (BG), Valle dei Foppi, $45,7317^{\circ} \mathrm{N} 10,0363^{\circ} \mathrm{E} 550 \mathrm{~m}, 13 . X \mathrm{XI} .1996$ leg. Cornali Gozzini (15 males, 4 females, CNSMB). Lombardia, Ardesio (BG)Valcanale, $45,9428^{\circ} \mathrm{N} 9,8565^{\circ} \mathrm{E} 1000 \mathrm{~m}, 30 . X \mathrm{XI} 2007$ leg. W. Zucchelli (5 males, CNSMB; 3 males, 1 female, OPC). Emilia Romagna, S. Sofia (FC), La Stretta, Fosso Abetio, $43.878^{\circ} \mathrm{N} 11.732^{\circ} \mathrm{E} 1200 \mathrm{~m}, 13 . X .1989$ leg. G. Campadelli (2 males, CNSMB). Emilia Romagna, S. Sofia (FC), La Stretta, Fosso Abetio, $43.878^{\circ} \mathrm{N} 11.732^{\circ} \mathrm{E} 1200 \mathrm{~m}$, 30.X.1989 leg. G. Campadelli (1 female, CNSMB). Toscana, Marradi, (FI), M.te Bruno Rio Canneto, $44,0259^{\circ} \mathrm{N}$ $11,6786^{\circ} \mathrm{E} 700 \mathrm{~m}, 15 . \mathrm{IX} .2003 \mathrm{leg}$. A. Usvelli (11 males, 2 females, CNSMB; 6 males, 2 females, OPC). Umbria, Spello (PG), Alto corso torrente Chiona, $43,03^{\circ} \mathrm{N} 12,71^{\circ} \mathrm{E}$ m350/500, 5.XI.1995 leg. Fucchi S., Salerno P. (1 male 1 female, $\mathrm{CC} \mathrm{n}^{\circ} 1750$ )

Remarks. Together with its closely related sibling species $M$. vestinorum they are subject to a separate genus status to distinguish from all the other species of the present genus Melampophylax s.l.

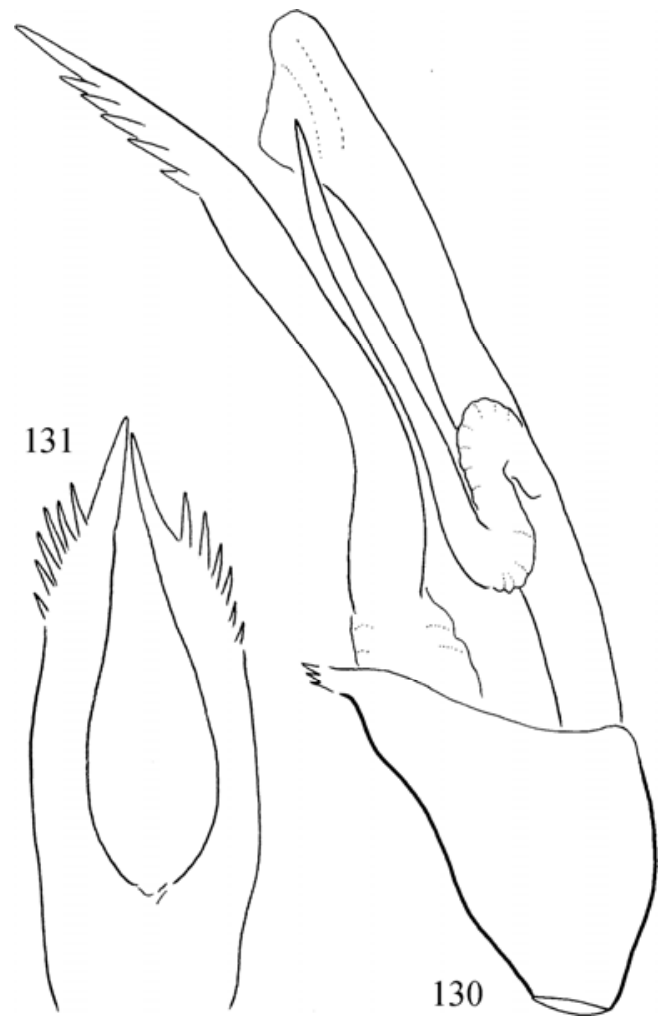

\section{Melampophylax vestinorum Moretti, 1991}

(Figures 132-134, 135-136)

Melampophylax vestinorum Moretti, 1991: 393.

Material examined. Italy, Calabria, Scilla (RC) m 1260, P. te S. Antonio, torr. Favazzina $38,187^{\circ} \mathrm{N} 15,846^{\circ} \mathrm{E}, 10$. XI. 2013, light trap leg. E. Castiglione, F. Manti \& P. Pantini, (2 females, CNSMB; 2 females, OPC). Lazio, Filettino (FR), Fonti Corore, Forno, $41,91^{\circ} \mathrm{N} 13,28^{\circ} \mathrm{E} 1100 \mathrm{~m}, 23 \mathrm{X} .1997$, leg. D. Serafini (1 female, CNSMB). Calabria Lorenzo Bellizzi (CS), torr. Vascello, $39,9101^{\circ} \mathrm{N} 16,2623^{\circ} \mathrm{E} \mathrm{m} 1000$, 30.XI.1994, tr. lum. leg. P. Pantini, M. Valle (1 male, CNSMB). Lorenzo Bellizzi (CS), torr. Vascello, 39,9101 ${ }^{\circ} \mathrm{N}$ $16,2623^{\circ} \mathrm{E} 1000 \mathrm{~m}, 30 . X .1994$, tr. lum. leg. P. Pantini, M. Valle (1 male, OPC).

\section{Melampophylax mucoreus species group}

Segment IX and gonopods not fused; gonopods not separated, touching mesad with well produced ventromesal plate; paraprocts form a simple pair of hook-shaped dorsal branches; position of cerci rather sagittal; aedeagus simple without any spine-like process, only lateral flange present to house the parameres; parameres simple elongated spine-like rod, without any spine-like modified setae. Very characteristic and species specific terminal blade evolved on the speciation trait of paramere in each species. This peculiar terminal configuration is a product of sexual coevolution. The pair of the internal tubes inside the elongated vaginal sclerite complex receives the elongated rod-shaped parameres during copulation. The terminal blade has crucial function to introduce the paramere shafts into the internal tubes inside the elongated vaginal sclerite complex during copulation. Vaginal dorsal sclerite complex is very much elongated to receive the parameres and the vaginal chamber is without any ventral sclerite. M. mucoreus, the most widely distributed species is probably the ancestral member of the group having the smallest elongation on the sclerite complex above the vaginal chamber, still near to plesiomorphic state. Inside the species group we distinguish the Melampophylax nepos species cluster.

Figures 130-131. Melampophylax melampus (McLachlan, 1876). $130=$ phallic organ in lateral view, $131=$ parameres in dorsal view. 


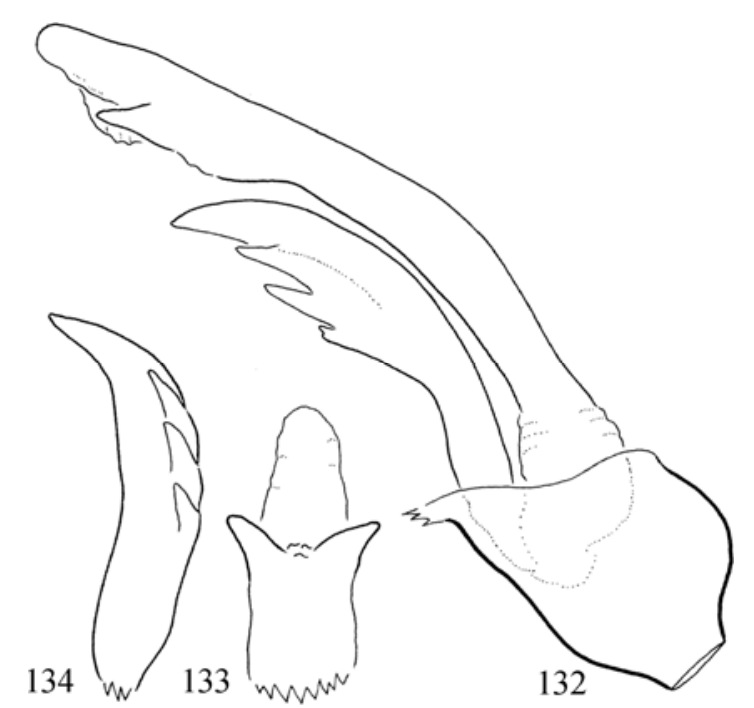

Figures 132-134. Melampophylax vestinorum Moretti, 1991. $132=$ phallic organ in lateral view, $133=$ head of aedeagus in ventral view, 134 = left paramere in dorsal view.

\section{Melampophylax altuspyrenaicus Botosaneanu, 1994}

(Figures 137-140)

Melampophylax altuspyrenaicus Botosaneanu, 1994: 363364.

Material examined. Holotype (male) and undesignated female: France, Pyrénées: Rivr Neste d'Aure in the neighbourhood of the lake Oredon, ca.1810-1880 m.a.s.l.; leg. H. Décamps- det. as M mucoreus (Hagen), Deposited in the Zoological Museum of Amsterdam. Lectotype. France, Hautes-Pyrénées, Estaing, Ilheou, 3.XI.2010, leg. G. Coppa, $(1 \hat{\jmath}$, OPC).

Remarks. We have examined the male holotype and the undesignated female specimen collected in the same habitat and deposited in the Zoological Museum of Amsterdam. Both the male and the female "specimens have cleared in $\mathrm{KOH}$ in a very unsatisfactory manner" (Botosaneanu 1994). Due to this over-cleared condition the male and female genitalia have lost all pigment, became almost invisible in any light direction. The genitalia have lost structure, almost totally collapsed. Therefore, in this study we have examined the available single intact male specimen collected nearby the locus typicus. Our drawings prepared from this recently collected additional specimen is similar to Botosaneanu's original drawings of the species description as regards the lateral view of the segment IX, cercus and paraproct and the lateral and ventral view of the gonopods. The speciation trait, the paramere is however strongly deformed on the holotype and the original drawing is not real. In the recently collected male specimen the paramere forms a rigid and long spine-like rod with shallow curvature in lateral view and almost straight in dorsal view. The enforced apical blade of the paramere is well produced, sagittally flattened, slightly upward directed in lateral view.

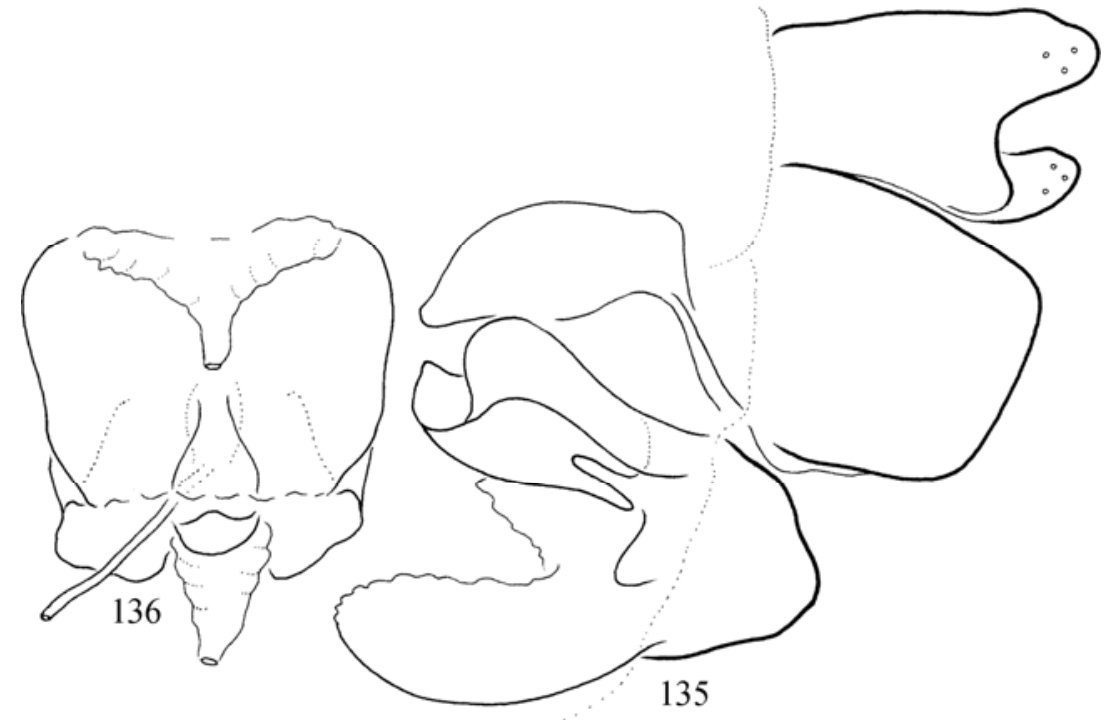

Figures 135-136. Melampophylax vestinorum Moretti, 1991. 135 = female genitalia with dorsal vaginal sclerite complex in left lateral view, $136=$ dorsal vaginal sclerite complex in dorsal view. 


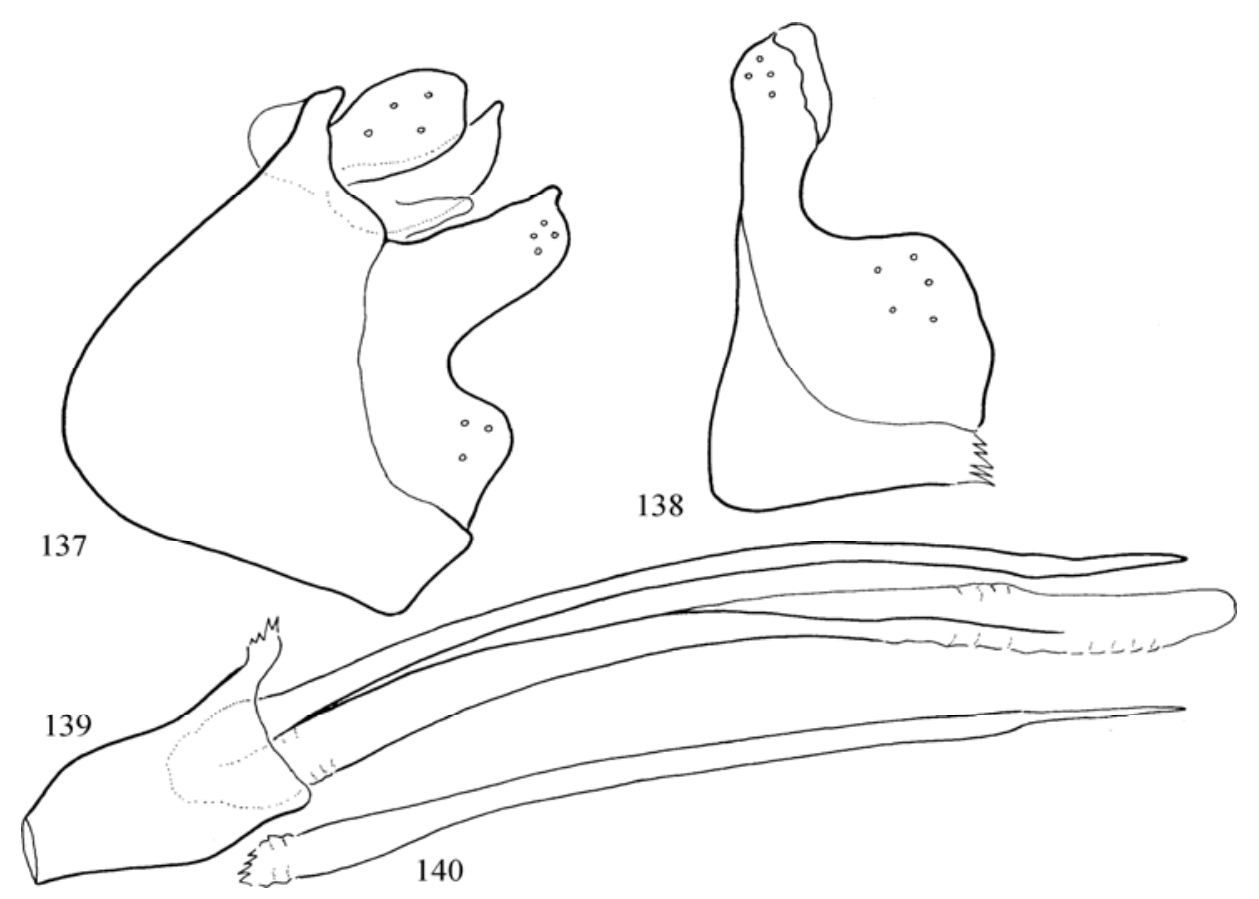

Figures 137-140. Melampophylax altuspyrenaicus Botosaneanu, 1994. $137=$ lectotype male genitalia in left lateral view $138=$ left gonopod in ventral view, $139=$ phallic organ in lateral view, $140=$ left paramere in dorsal view.

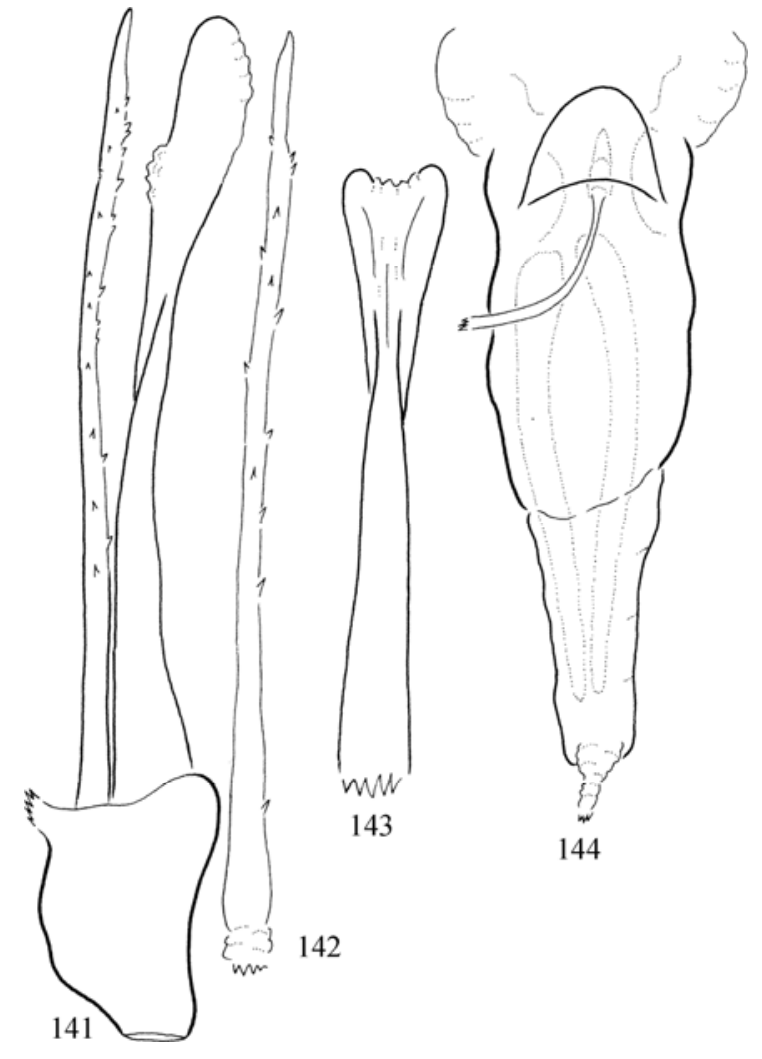

Melampophylax cantalicus Botosaneanu, 1994

(Figures 141-144)

Melampophylax cantalicus Botosaneanu, 1994: 364-365.

Material examined. France, Puy-de-Dôme, Chambon, Ru en Amont de la Croix Morand, 11.X.2007, leg. G. Coppa, $(2 \hat{\jmath}, 2 \propto$ OPC $)$.

Remarks. Easily distinguished from all other species by the paramere covered with numerous spicules and by the shape of the elongation of the vaginal sclerite complex. Such unique apomorphic trait of small triangular teeth or spicules was detected on the aedeagus stem at the Allogamus silanus species seems endemic to Calabria (Oláh et al. 2014).

\section{Melampophylax banaticus Botosaneanu, 1995 stat. nov.}

(Figures 145-148)

Melampophylax nepos ssp. banaticus Botosaneanu, 1995: 76. Melampophylax nepos ssp. banaticus Botosaneanu, 1995: Malicky 2005: 577.

Figures 141-144. Melampophylax cantalicus Botosaneanu, $1994.141=$ phallic organ in lateral view, $142=$ left paramere in dorsal view, $143=$ aedeagus in ventral view, $144=$ dorsal vaginal sclerite complex in dorsal view. 
Material examined. Romania, Caraş-Severin county, Țar$\mathrm{cu}$ Mts., open brook on the $\mathrm{W}$ slope of Mt. T,arcu, N45 17’30.9”, E22³0'59.9”, 1770 m, 14.10.2011, Á. Ecsedi, T. Kovács \& G. Puskás (4 males, 2 females; OPC). Retezat Mts. Gura Apelor, N45.33 E22.88, 1500 m, 20.X. 2007 leg. M. Bálint, E. Magyari \& M. Braun (1 male, OPC).

Remarks. This is an incipient sibling species closely related M. polonicus and M. gutinicus, but differs from both by having female with differently shaped vaginal sclerite elongation and males with pointed mesal corner on the mesal plate of the gonopods. Also differs by having the speciation trait, the paramere very stout with shallow curvature and with pronounced mace-like terminal blade.

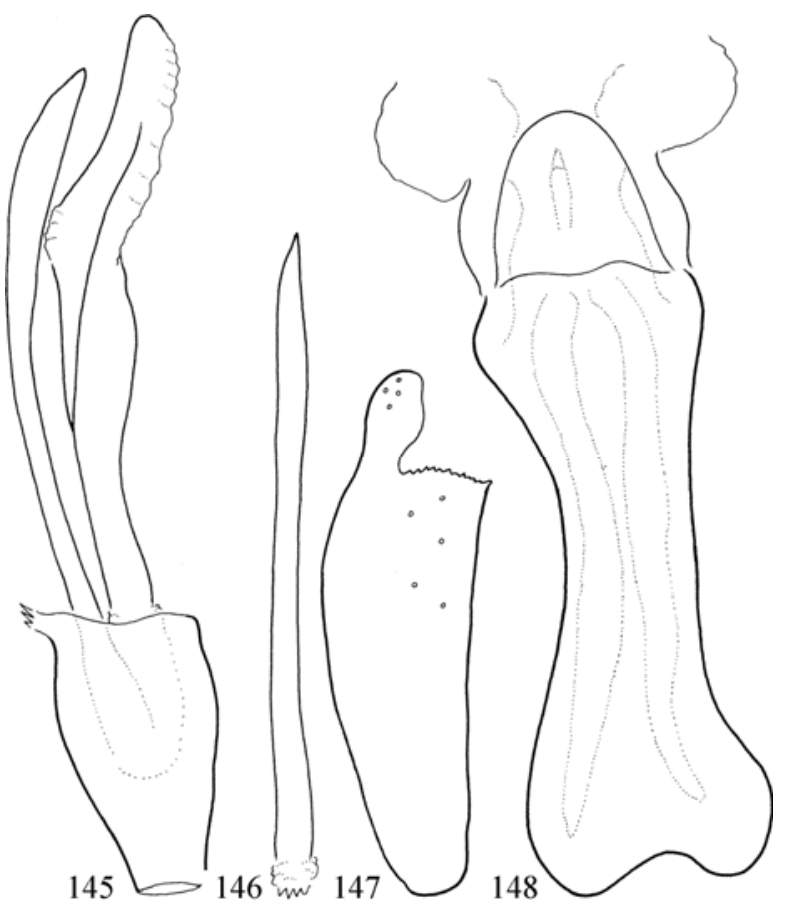

Figures 145-148. Melampophylax banaticus Botosaneanu, 1995. $145=$ phallic organ in lateral view, $146=$ left paramere in dorsal view, $147=$ left gonopods in ventral view, $148=$ dorsal vaginal sclerite complex in dorsal view.

\section{Melampophylax gutinicus Botosaneanu, 1995 stat. nov.}

(Figures 149-152)

Melampophylax nepos ssp. gutinicus Botosaneanu, 1995: 7576.
Melampophylax nepos ssp. gutinicus Botosaneanu, 1995: Malicky 2005: 577.

Material examined. Romania. Maramures county, Muntii Ignis, Deseşti-Stațiunea Izvoare, open brook on the Valhani Plateau, 1020m, N47²3.015' E23 ${ }^{\circ} 44.547^{\prime}, 07.10 .2010$, leg. P. Barcánfalvi, D. Murányi \& J. Oláh, (5 males, 2 females, OPC). Maramures county, Muntii Ignis, Desești-Stațiunea Izvoare, open stream on the Valhani Plateau, $940 \mathrm{~m}, \mathrm{~N} 47^{\circ}$ 43.945' E23ํ⒋661', 08.10.2010, leg. P. Barcánfalvi, D. Murányi \& J. Oláh, (1 male, OPC). Maramureş county, Muntii Ignis, Desesti-Statiunea Izvoare, spring brook on the Valhani Plateau, 955m, N4744.177' E234․ ${ }^{\circ}$.971', 08.10.2010, leg. P. Barcánfalvi, D. Murányi \& J. Oláh, (2 males, OPC; 2 males, CSNMB). Radnei Mts. Rodnei Mts. small tributary just below Iza Spring, Albastru al Izei, 1020m, 27. IX. 2014, leg. J. Oláh \& Cs. Balogh (1 male, OPC).

Remarks. Incipient sibling species closely related M. banaticus and M. polonicus, but differs from both by having female with differently shaped vaginal sclerite elongation, that is short and narrowing distad and the male has the most rounded mesal corner on the mesal plate of the gonopods as well as very slender paramere that has minute terminal blade.

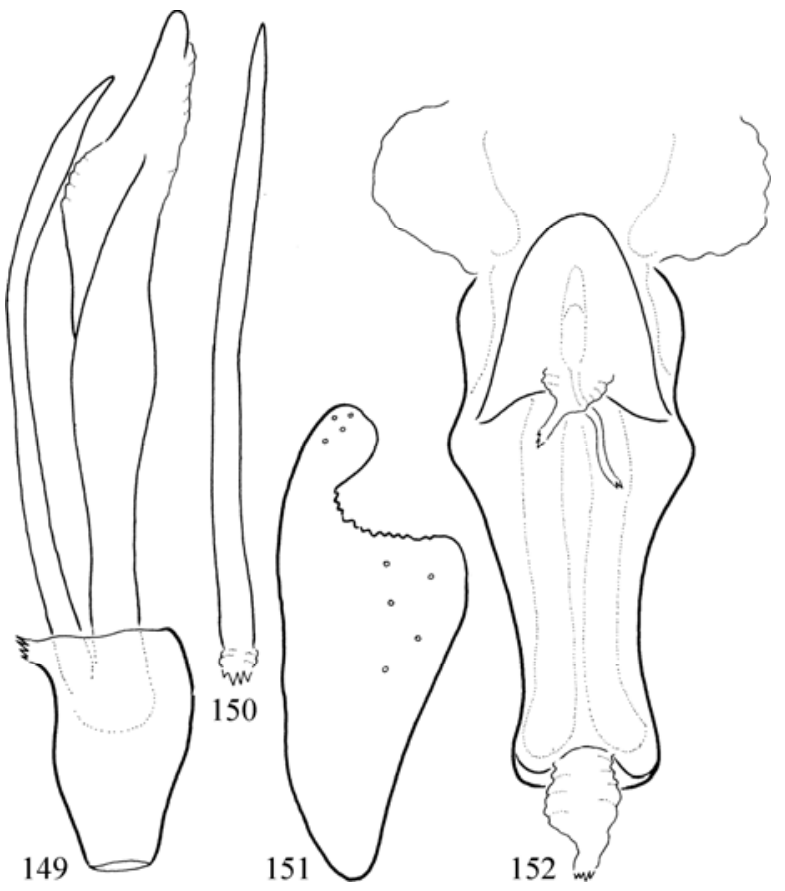

Figures 149-152. Melampophylax gutinicus Botosaneanu, 1995. $149=$ phallic organ in lateral view, $150=$ left paramere in dorsal view, 151 = left gonopods in ventral view, $152=$ dorsal vaginal sclerite complex in dorsal view. 
Melampophylax keses Coppa \& Oláh, sp. nov.

(Figures 153-157, 158-160)

Diagnosis. This new species is close to and diverged from M. mucoreus, but differs by having paramere more developed the terminal blade is flattened coronally, not sagittally and differs also by the more elongated vaginal sclerite complex.

Material examined. Holotype. France, Alpes-de-HauteProvence, Uvernets Fours, Braissette zone humide, contrebas du sentier, 20.X.2009, leg. G. Coppa, (1 $\hat{\jmath}^{2}$, CPC). Allotype. Same as holotype (1 female, CPC).

Male genitalia. Segment IX convex anterad, deep concave posterad. Cerci rounded triangular. Paraproct hook-shaped in lateral view with rather produced accessory process laterad on the ventral branch. Gonopods with mesal plate, but without mesal elongation. Paramere forming an elongated spine-like rod with specific terminal blade. Aedeagus with well developed lateral flanges.

Female genitalia. Tergum IX bilobed in dorsal view with shallow V-shaped mesal excision; basal region broader triangular. Tergum $\mathrm{X}$ less sclerotized and slightly protruding and excised mesad as visible both in dorsal and ventral view. Lateral setose lobe, the sternite of segment IX quadrangular in lateral view. The elongated vaginal sclerite narrowing distad.

Etymology. Epithet keses, from "késes" armed with knife, refers to the shape of the terminal blade on the paramere.

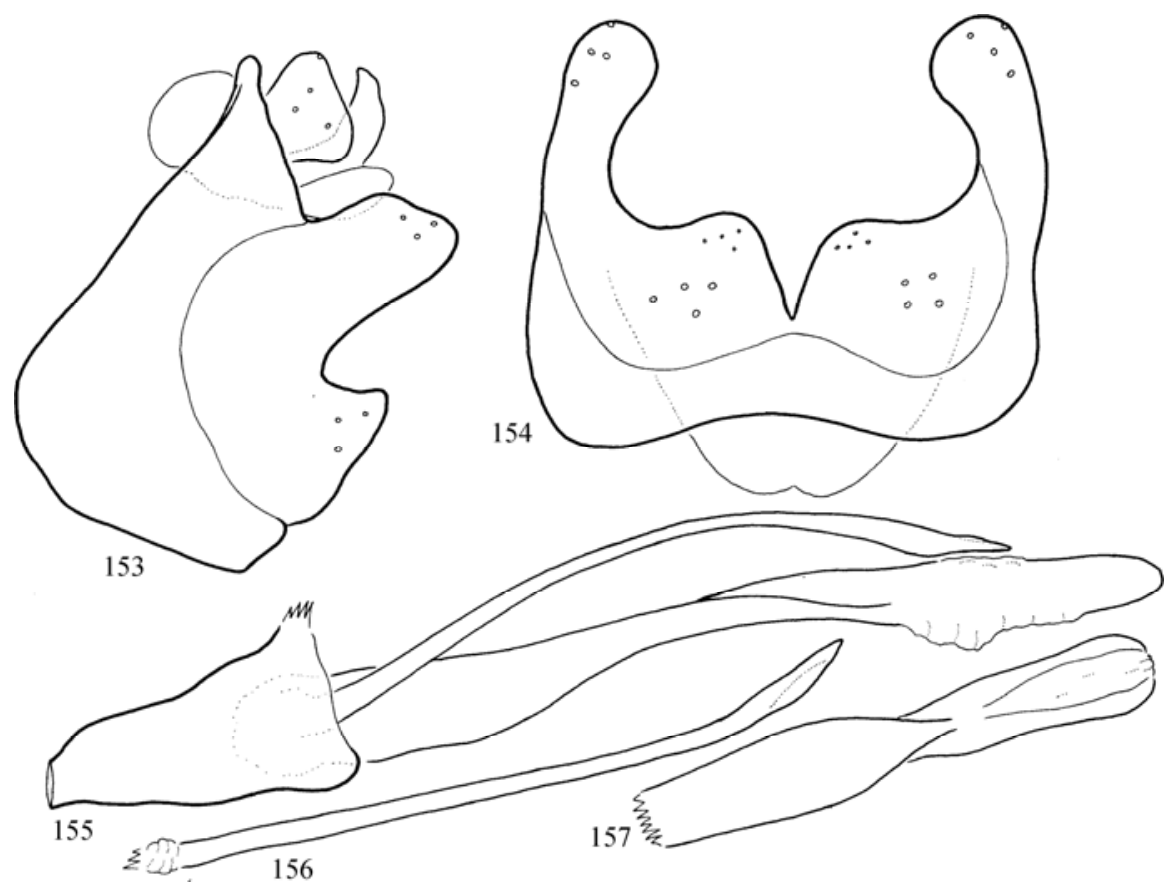

Figures 153-157. Melampophylax keses Coppa \& Oláh, sp. nov. $153=$ male genitalia in lateral view, $154=$ gonopods in ventral view, 155 = phallic organ in lateral view, $156=$ left paramere in dorsal view, $157=$ aedeagus in ventral view.

\section{Melampophylax mucoreus (Hagen, 1861)}

(Figures 161-169)

Halesus mucoreus Hagen, 1861: 115.

Material examined. England, Horton in Ribblesdale, SD8072, North Yorkshire 12.X.1973, leg. A. Brindle, (5 male, 1 female; 34/814724, F3298.3311, MMUE). River Wharfe, Grass Woods, Grassington, SE0064, North Yorkshire, 7.X.1975, leg. A. Brindle, (1 female; 34/981662, F3298.3309, MMUE). Ings Beck, near River Ribble, Downham, SD7844, Lancashire, 5.X.1975, leg. A. Brindle, (1 female; 34/775455, F3298.3310, MMUE). France, Doubs, Mouthe, Doubs aval résurgence, 24.IX.2009, leg. G. Coppa, $(3 \AA, 2 \bigcirc ;$ OPC). France, Haute-Marne, Orquevaux, Cul du Cerf/étang du Moulin, 27. IX. 2009, leg. G. Coppa, $(1 \hat{\jmath}, 1$; 
OPC). France, Ardennes, Signy-l'Abbaye, Source du Gibergon, 9.X.2006, leg. G. Coppa, France, Ardennes, Signyl'Abbaye, résurgence de la Fosse Bleue à Librecy, 25.X. 2008, leg. G. Coppa, (2犬̂,3; OPC; 1 male, 1 female; CSNMB).

Remarks. Easily distinguished from all the o- ther species by having the most short vaginal sclerite elongation and the slimmest paramere with very long, but tiny terminal blade. The short elongation and the slim paramere is a possible sign of the sexual coevolution.

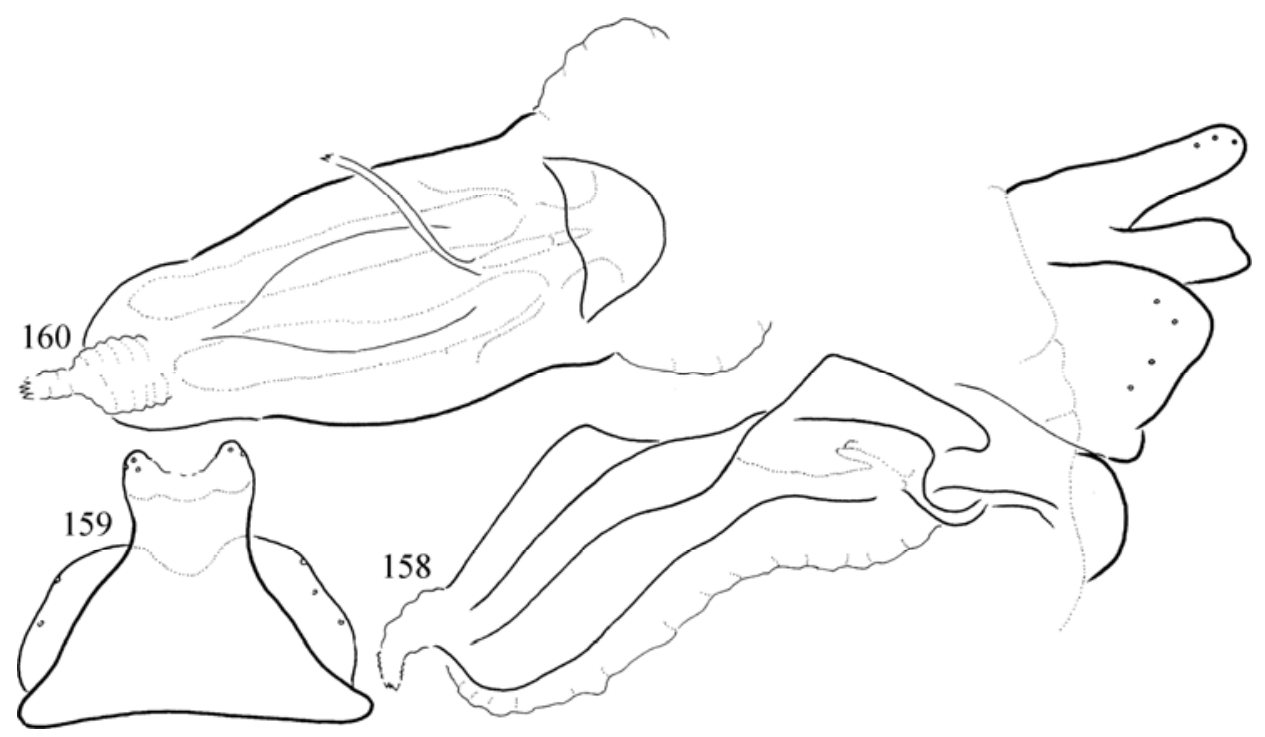

Figures 158-160. Melampophylax keses Coppa \& Oláh, sp. nov. 158 = female genitalia with dorsal vaginal sclerite complex in left lateral view, $159=$ female anal tube in dorsal view, $160=$ dorsal vaginal sclerite complex in dorsal view.

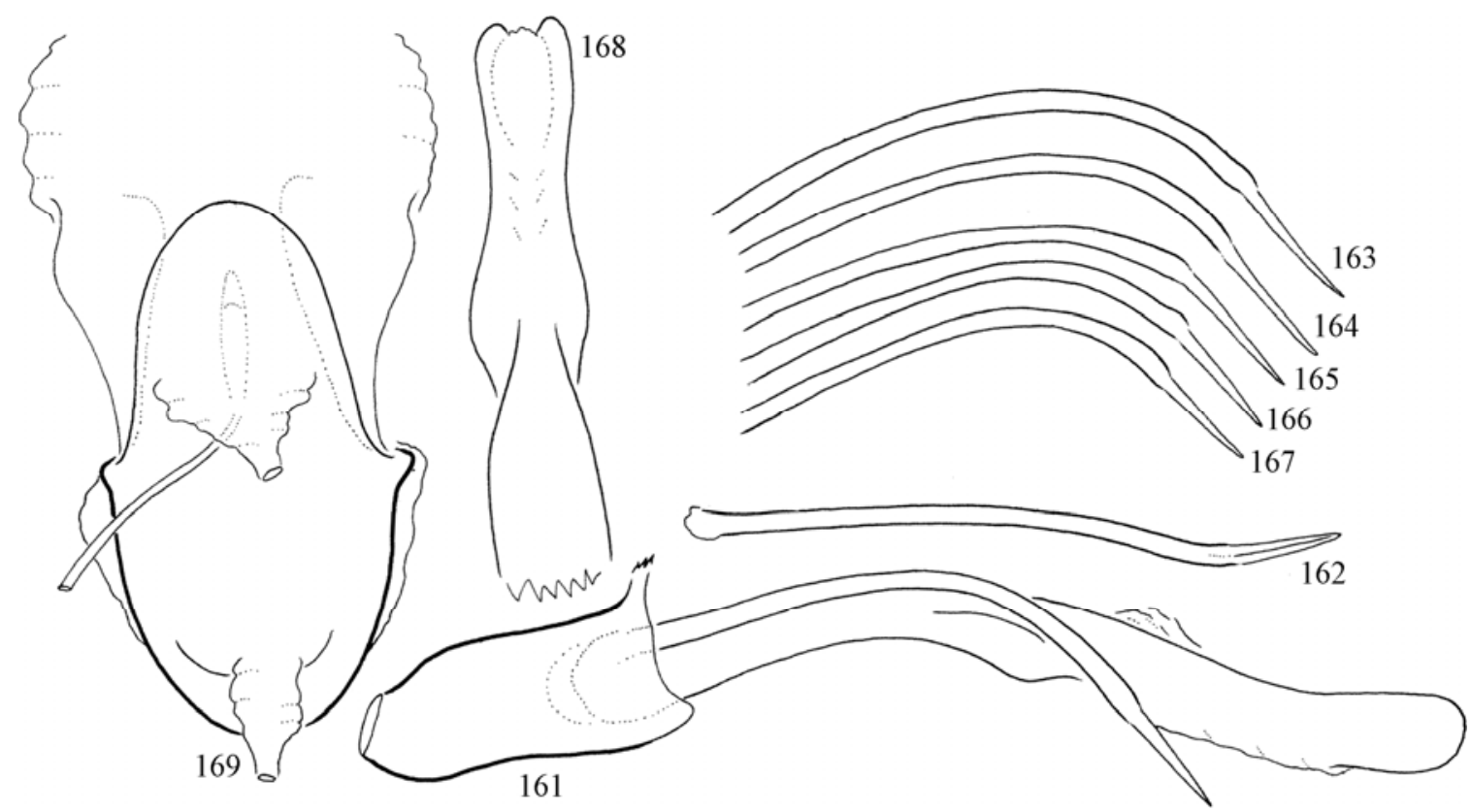

Figures 161-169. Melampophylax mucoreus (Hagen, 1861). $161=$ phallic organ in lateral view from France, $162=$ left paramere in dorsal view, 163-167 = left parameres from England in lateral view, $168=$ aedeagus in ventral view, $169=$ dorsal vaginal sclerite complex in dorsal view. 


\section{Melampophylax polonicus Malicky, 1990}

(Figurers 170-173)

Melampophylax polonicus Malicky, 1990: 8-9.

Material examined. Poland, Bieszczady Mts. X. (October, without day and year), leg. det. B. Szczęsny (2 males, 1 female; OPC).

Remarks. Incipient sibling species closely related M. banaticus and M. gutinicus. Differs from both by having female with differently shaped vaginal sclerite elongation with produced subapical constriction; males with straight truncate apical margin on the mesal plate of the gonopods as well as long and strong paramere that has strong terminal blade.

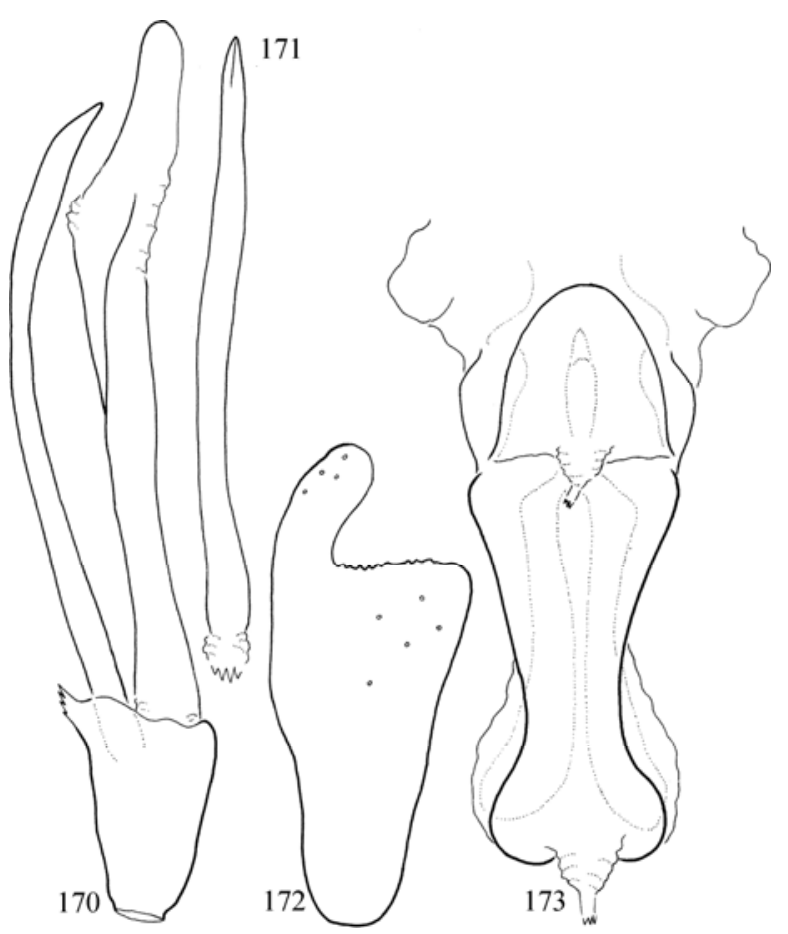

Figures 170-173. Melampophylax polonicus Malicky, 1990. $170=$ phallic organ in lateral view, $171=$ left paramere in dorsal view, $172=$ left gonopods in ventral view, $173=$ dorsal vaginal sclerite complex in dorsal view.

\section{Melampophylax nepos new species cluster}

This species cluster is characterized by mesal elongation of the ventromesal plate with straight, mesad or laterad curving pointed or blunt apex, absent in other species of the M. mucoreus species group. The parameres are species specific and differ in the robustness, curvature and in the formation of the terminal blade. The basal architecture and gross morphology of paramere is identical, but the divergences in its fine structure are very consistent and stable between populations and on a large distributional area. The unique mesal elongation of the gonopods is an auxiliary speciation trait also developed species specific shapes in the sexual selection processes. In sexual coevolution the elongation of the vaginal sclerite complex produced species specific shapes.

\section{Melampophylax austriacus Malicky, 1990}

(Figures 174-176, 177-178)

Melampophylax austriacus Malicky, 1990: 8.

Material examined. Austria, Schwarze Sulm, 20. X. 2013 leg. W. Graf (1 male, 1 female; OPC).

Remarks. Auxiliary mesal elongation of the gonopods blunt broad triangular. Terminal blade on paramere sagittally flattened. Elongated vaginal sclerite complex broad basad and narrowing distad.

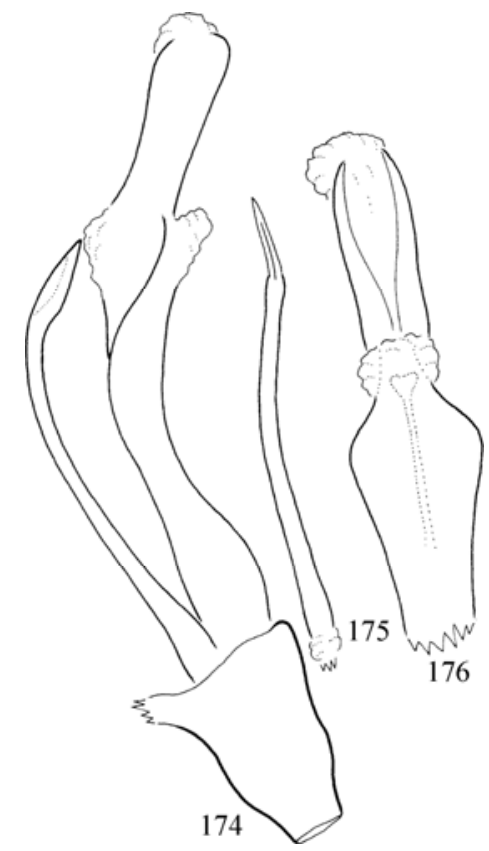

Figures 174-176. Melampophylax austriacus Malicky 1990. $174=$ phallic organ in lateral view, $175=$ left paramere in dorsal view, $176=$ aedeagus in ventral view. 


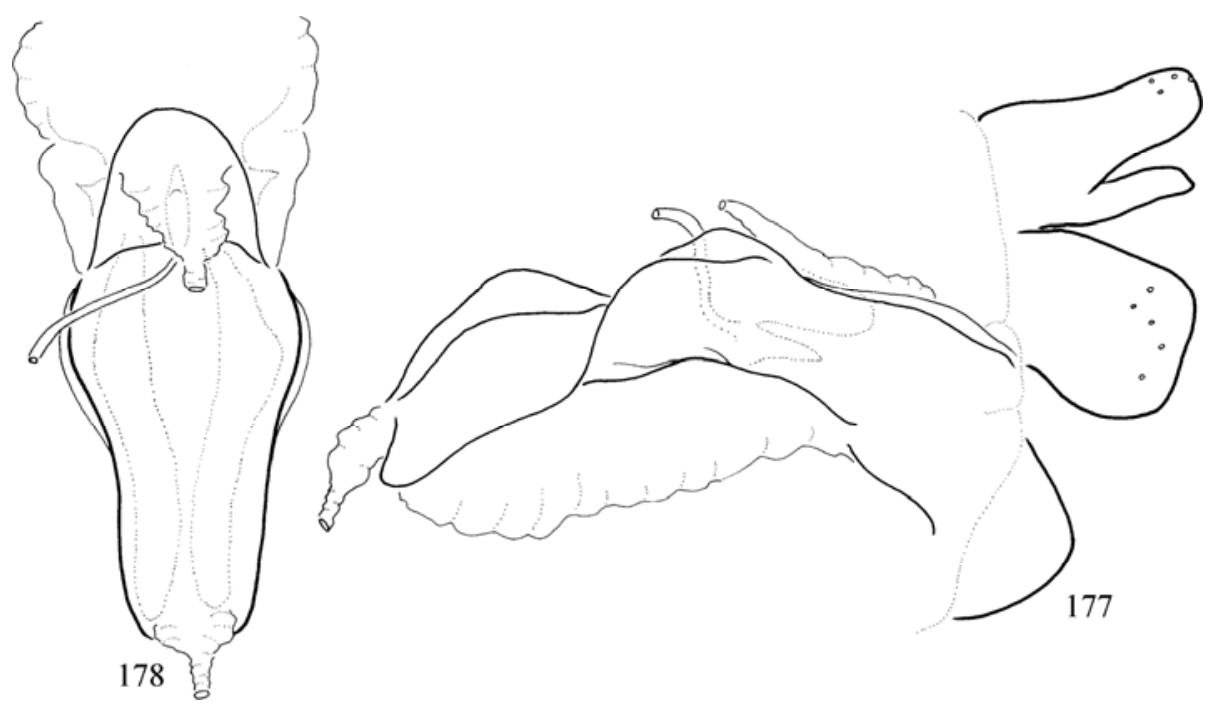

Figures 177-178. Melampophylax austriacus Malicky, 1990. 177 = female genitalia with dorsal vaginal sclerite complex in left lateral view, $178=$ dorsal vaginal sclerite complex in dorsal view.

\section{Melampophylax nepos (McLachlan, 1880)}

(Figures 179-192, 193-206, 207)

Halesus nepos McLachlan, 1880: 40.

Material examined. Hungary. Bükk Mts. Sebes Stream (Sebes Víz), 7.X.1964, singled leg. J. Oláh (20 males, OPC). Slovakia. Svermovo, Hron Spring, 12.X.1989, leg. S. Nógrádi \& Á. Uherkovich (2 males, 1 female; OPC). West Tatra, River Bela, VII. 1978, light trap (1 male, OPC). Strbské Pleso, 14.X.1989, leg. S. Nógrádi \& Á. Uherkovich (1 female; OPC). Pribilina, Hrdovo, 14.X.1989, leg. S. Nógrádi \& Á. Uherkovich (1 male; OPC). Vernar, Kopanec, 9.X.1987, leg. Á. Uherkovich (2 males, 1 female; OPC). Stratená, Dobsinská Ladova Jaskyna, 26.IX.1984, leg. S. Nógrádi (3 males, 1 female; OPC). Stratená, Pálenica, 9.X.1987, leg. Á. Uherkovich (2 males, 2 females; OPC). Dobsiná, Dankova, 13.X.1989, leg. Á. Uherkovich (1 male, 1 female; OPC). Stratená, valley toward Hrabusice, 26.IX.1984, leg. Á. Uherkovich (1 male, 1 female; OPC). Rejdova (Sajóréde), Slana (Sajó) stream, below spring, N48 47'6"' E20 ${ }^{\circ} 12^{\prime} 18^{\prime}$ ', 1120 m, 3. X. 2013, singled leg. J. Oláh \& J. Kecskés (2 males, 2 females; CSNMB). Rejdova (Sajóréde), right tributary of Slana (Sajó) stream, lower reach, N48 $48^{\prime} 53^{\prime}$ " E20 $15^{\prime} 51^{\prime}$, 680 m, 3. X. 2013, singled leg. J. Oláh \& J. Kecskés J. (2 males, 1 female; OPC). Rejdova (Sajóréde), right tributary of Slana (Sajó) stream, lower reach, N48 $48^{\prime} 53^{\prime \prime}$ E20 ${ }^{\circ} 15^{\prime} 51$ ', $680 \mathrm{~m}, 3$. X. 2013, singled leg. J. Oláh \& J. Kecskés (2 females, OPC). Rejdova (Sajóréde), Mlynna stream, below spring, at bridge, N48 46'16" E20 $0^{\circ} 13^{\prime} 31$ '" $1250 \mathrm{~m}$, 3. X. 2013, singled leg. J. Oláh \& J. Kecskés J. (8 males, 31 females, OPC). Rejdova (Sajóréde), right tributary of Mlynna stream, at bridge, N48 46'16" E20 13 '31'" $1250 \mathrm{~m}$, 3. X. 2013, singled leg. J. Oláh \& J. Kecskés (1 male, 5 females; OPC). Banskobystrický region, Pol'ana Mts, Hriňová, Bystré, spring brook of Bystrý Stream, N48 37.569' E19²9.261', 1025m 8.X.2013, singled leg. J. Oláh \& L. Szél (8 males, 4 female; OPC).
Remarks. Auxiliary mesal elongation of the gonopods with mesad turning apex. Paramere with most shallow curvature in the complex; the terminal blade of the paramere rounded and slender, not flattened like at M. szczesnyorum and not robust like at $M$. triangulifera. Elongated vaginal sclerite complex narrowing distad.

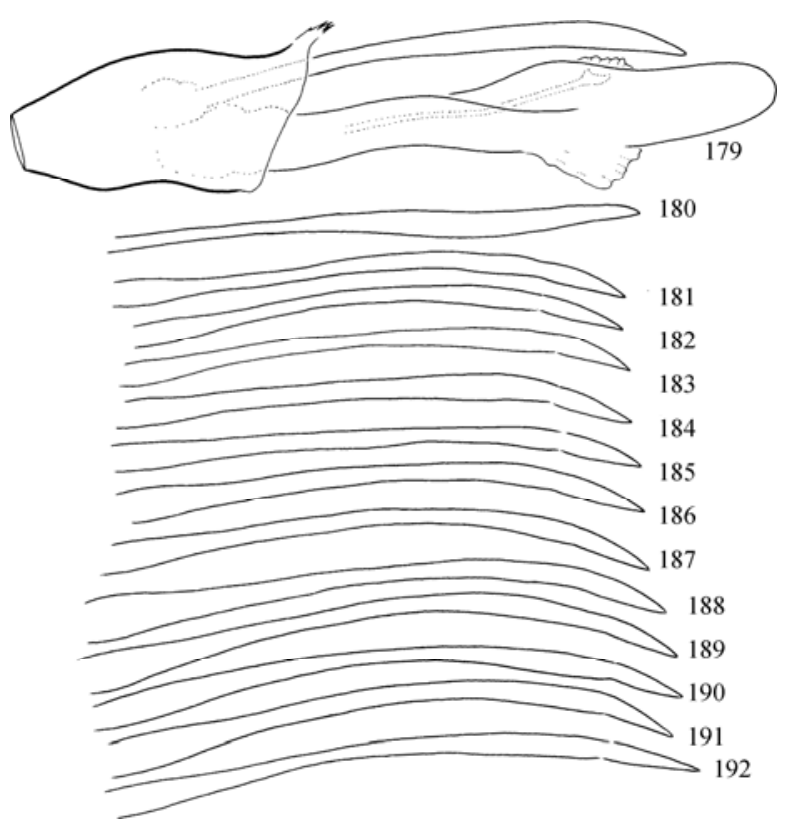

Figures 179-192. Melampophylax nepos (McLachlan, 1880). $179=$ phallic organ in lateral view, $180=$ left paramere in dorsal view, $181-187=$ left paramere from Hungary in lateral view, 188-192 = left paramere from Slovakia in lateral view. 

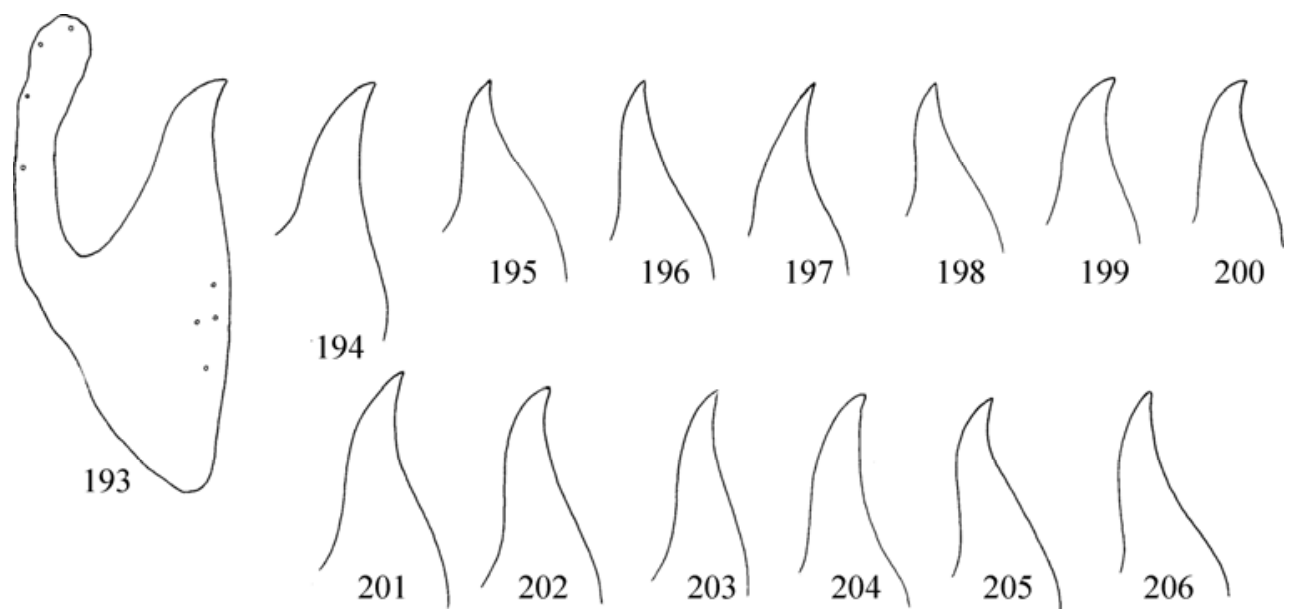

Figures 193-206. Melampophylax nepos (McLachlan, 1880). 193 = left gonopods with mesal elongation from Hungary in lateral view, 194-200 = mesal elongation of gonopod from Hungary in ventral view, 201-206 = mesal elongation of gonopod from Slovakia in lateral view.

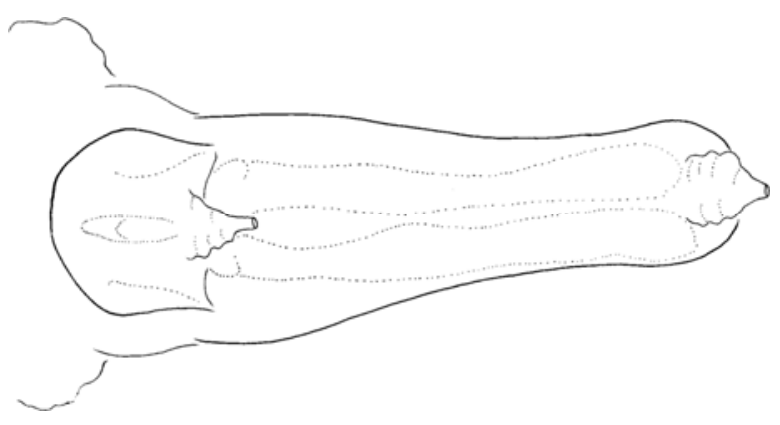

Figure 207. Melampophylax nepos (McLachlan, 1880). Dorsal vaginal sclerite complex in dorsal view.

\section{Melampophylax szczesnyorum Oláh \& Chvojka,} sp. nov.

(Figures 208-220, 221-234, 235-239)

Melampophylax nepos triangulifera Botosaneanu, 1957. Misidentification (Szczęsny 1980)

Diagnosis. This new species differs from its close relatives, from the sibling species of $M$. nepos and M. triangulifera by having the auxilliary mesal elongation of the gonopods with straight apex, mesad turning at $M$. nepos and laterad turning at $M$. triangulifera; the terminal blade of the paramere produced into a very flattened shape, a unique and powerful device in copulation; the elongated vaginal sclerite complex broadening distad. Only historical material was available for this study. No newly collected material!
Material examined. Holotype. Ukraine, original label: "Chomiak, (pot. podziemny), 6.X.1905, leg. Dziędzielewicz (1 male, Klapálek's Collection in NMPC: No. 50). Allotype. Ukraine, original label: "Chomiak. p. Weredyk, 1. 10.1907, leg Dz." (1 female, Dziędzielewicz's collection in NHMISEA). Paratypes. Ukraine, original label: "Tatarów (Prutec), 7.-10.-1905 Dz" (1 male, Dziędzielewicz's collection in NHM-ISEA). Ukraine, original label: "Chomiak, p. Weredyk, 1.-10.-1907 Dz" (1 male, Dziędzielewicz's collection in NHM-ISEA). Ukraine, original label: "Chomiak (pot. podziemny), 6.-10.-1905 Dz" (1 male, Dziędzielewicz's collection in NHM-ISEA). Ukraine, original label: "Tatarów, (Błotek.) X. 1906" (1 male, Dziędzielewicz's collection in SMNHL: No. 1385). Ukraine, original label: "Czarnohora, Foreszczynka 4.X. 1910” (1 male, Dziędzielewicz's collection in SMNHL: No. 1399). Ukraine, original label: "Czarnohora, Foreszczynka 19.X. 1910" (1 male, Dziędzielewicz's collection in SMNHL: No. 1402). Ukraine, original label: "Czarnohora, Kozmieska 16.X. 1908" (1 male, Dziędzielewicz's collection in SMNHL: No. 1396). Ukraine, origial label: "Chomiak, Barani. 9.X. 1907" (1 female, Dziędzielewicz's collection in SMNHL: No. 1389). Ukraine, original label: "Chomiak, Prutec. 2.X. 1912" (1 female, Dziedzielewicz's collection in SMNHL: No. 1403). Ukraine, original label: "Chomiak, Prutec. 2.X. 1912" (1 male, Dziędzielewicz's collection in SMNHL: No. 1401). Ukraine, original label: "Chomiak, P. Weredyk. 7.X. 1907" (1 female, Dziędzielewicz's collection in SMNHL: No. 1386). Ukraine, original label: "Czarnohora, Foreszczynka 4.X. 1910" (1 male, Dziędzielewicz's collection in SMNHL: No. 1397). Ukraine, original label: "Chomiak, Gnilec. 5.X. 1907" (1 male, Dziędzielewicz's collection in SMNHL: No. 1394). Ukraine, original label: "Chomiak, P. Weredyk. 23.IX. 1907" (1 male, Dziędzielewicz's collection in Dziędzielewicz's collection in SMNHL: No. 1388). Ukraine, original label: "Chomiak, (Blotek.) 22. IX.1906" (1 female, Dziędzielewicz's collection in SMNHL: No. 1392). Ukraine, original label: "Chomiak, Potok Barani. 18.X. 1907" (1 female, Dziędzielewicz's collection in SMNHL: No.1384). 
Male genitalia. Segment IX convex anterad, deep concave posterad. Cerci rounded triangular. Paraproct hook-shaped in lateral view with produced accessory process laterad on the ventral branch. Gonopods with mesal plate, with mesal elongation. This auxilliary mesal elongation of the gonopods is straight, slightly directed laterad in some specimens. Paramere forms an elongated spine-like rod with specific terminal blade. Terminal blade of the paramere significantly flattened sagittaly. Aedeagus with well developed lateral flanges.

Female genitalia. Tergum IX bilobed in dorsal view with shallow V-shaped excision; basal region broader triangular. Tergum $\mathrm{X}$ less sclerotized and slightly protruding and excised mesad as visible both in dorsal and ventral view. Lateral setose lobe, the sternite of segment IX quadrangular in lateral view. Elongated vaginal sclerite complex broadening distad and bilobed.

Etymology. We dedicated this species to Dr. Bronislav Szczesny and his wife, who has produced significant contribution to the knowledge of the Trichoptera in the North-East Carpathians covering both Poland and Ukraine.

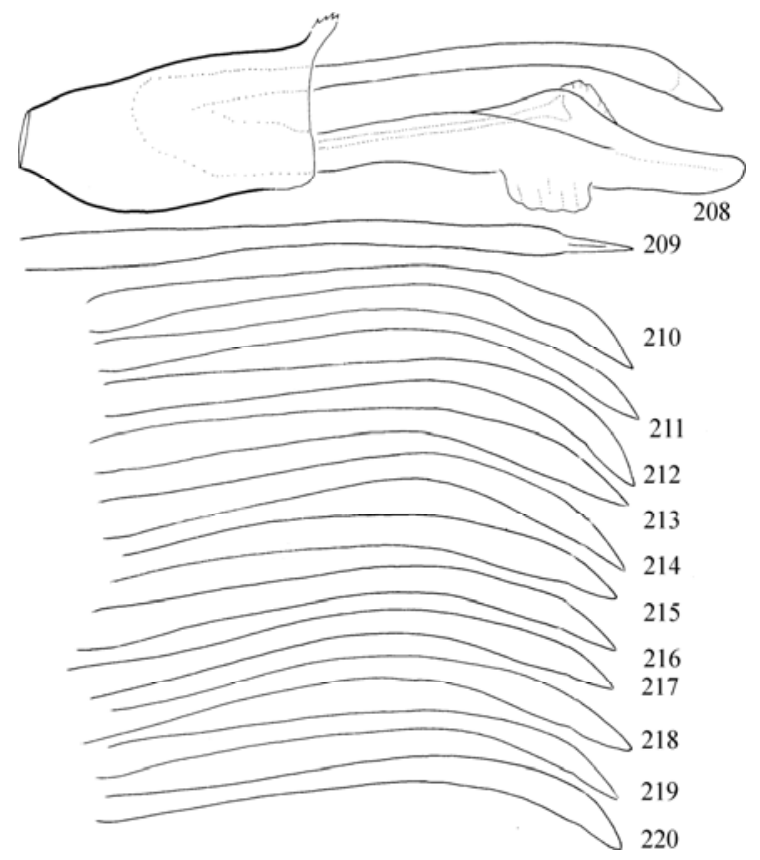

Figures 208-220. Melampophylax szczesnyorum Oláh \& Chvojka, sp. nov. $208=$ phallic organ in lateral view, $209=$ left paramere in dorsal view, 210-220 = left paramere in lateral view.

\section{Melampophylax triangulifera Botosaneanu, 1957 stat. nov.}

(Figures 240-251, 252-263, 264-275)

Melampophylax nepos triangulifera Botosaneanu, 1957: 400-401.

Melampophylax nepos triangulifera Botosaneanu, 1957: Malicky 1990: 2.

Melampophylax nepos triangulifera Botosaneanu, 1957: female described, Botosaneanu 1995: 74-75, 76, fig. 41-44.

Melampophylax nepos triangulifera Botosaneanu, 1957: Malicky 2005: 576

Material examined. Romania, Eastern Carpathians, Hargitha Mts., springs and streamlets between Baile Hargitha and Cabana Madaras, 1650-1700 m, 14. X. 1970 leg. L. Botosaneanu (6 males, OPC). Gurghiu Mts. near Bucin Pass,

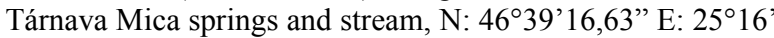
42,46”, 1290, 30.X.2014, leg. Z. Baczó, Cs. Balogh, J. Kecskés \& J. Oláh. (39 males, 4 females; OPC; 10 males, 2 females; CSNMB). Gurghiu Mts. near Bucin Pass, Gainasa springs and stream, N: 46 $40^{\prime} 11,35^{\prime \prime} \mathrm{E}: 2^{\circ} 17^{\prime} 39,06^{\prime}, 1400$, 30.X.2014, leg. Z. Baczó, Cs. Balogh, J. Kecskés \& J. Oláh (1 male, 1 female; OPC). Hargitha Mts. Filio stream side spring, N: 46²7’03,90” E: 2533'29,29”, 1350m, 31.X.2014 leg. Z. Baczó, Cs. Balogh, J. Kecskés \& J. Oláh. (1 male, 3 females; OPC). Caliman Mts. Fantanele stream, N: 46 ${ }^{\circ} 59^{\prime}$ 04,00” E: 2505’52,56”, 776m, 1.XI.2014, leg. Z. Baczó, Cs. Balogh, J. Kecskés \& J. Oláh. (3 females; OPC).

Remarks. This is a sibling species of M. nepos and M. szczesnyorum. Auxilliary mesal elongation of the gonopods laterad curving. Terminal blade of the paramere robust and rounded. Elongated vaginal sclerite complex rather variable inside populations.

\section{Genus Rhadicoleptus Wallengren, 1891}

Type species: Stenophylax alpestris Kolenati (monobasic)

Transferred here from Limnephilini to Stenophylcini tribe.

Schmid (1955) has placed the genus Rhadicoleptus with the species alpestris, spinifer and ucenorum in his newly created tribe Limnephilini with some uncertainty. He has recognized that the genus Rhadicoleptus is rather far from Limnephilus, moreover the distinction between Limnephilini and Stenophylacini tribes remained unclear. The characters of the anastomose disposition on forewing and the massive genitalia, 


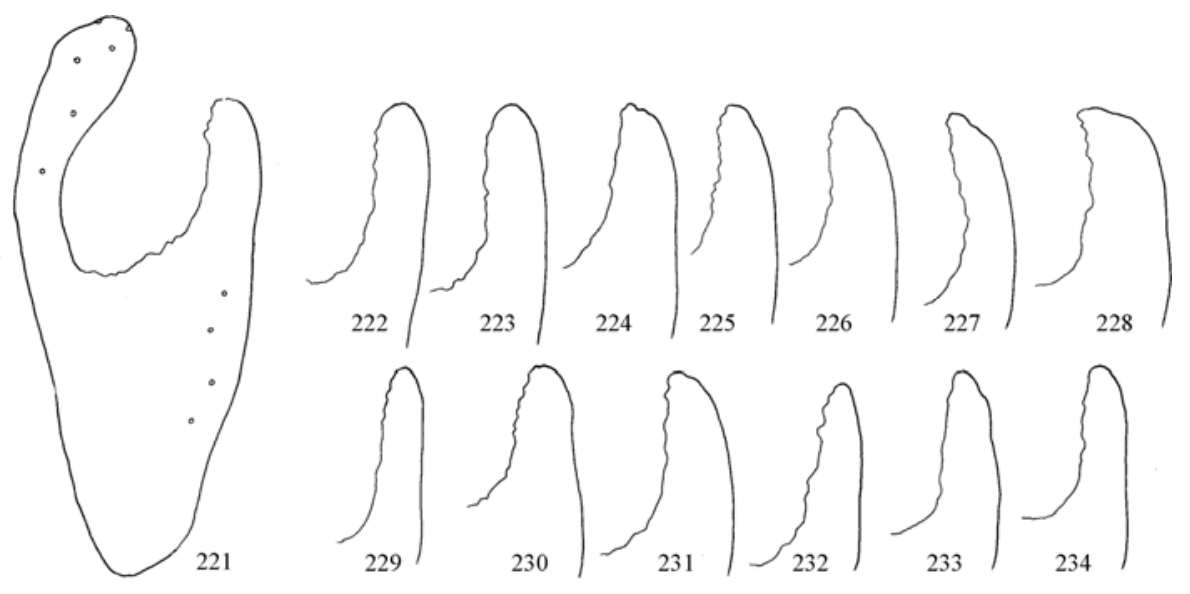

Figures 221-234. Melampophylax szczesnyorum Oláh \& Chvojka, sp. nov. 221 = left gonopods with mesal elongation in lateral view, $222-234=$ mesal elongation of gonopod in ventral view.

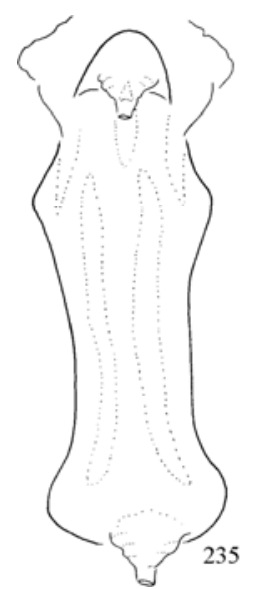

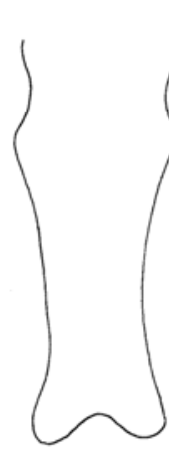

236

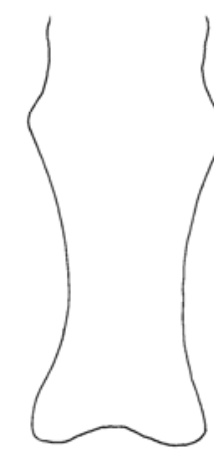

237

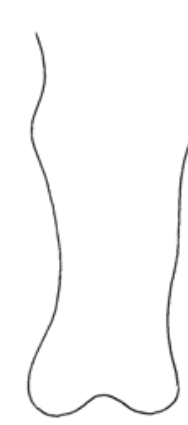

238

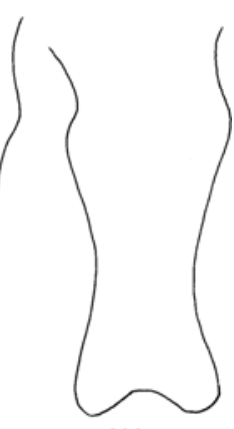

239

Figure 235-239. Melampophylax szczesnyorum Oláh \& Chvojka, sp. nov. Dorsal vaginal sclerite complex in dorsal view.

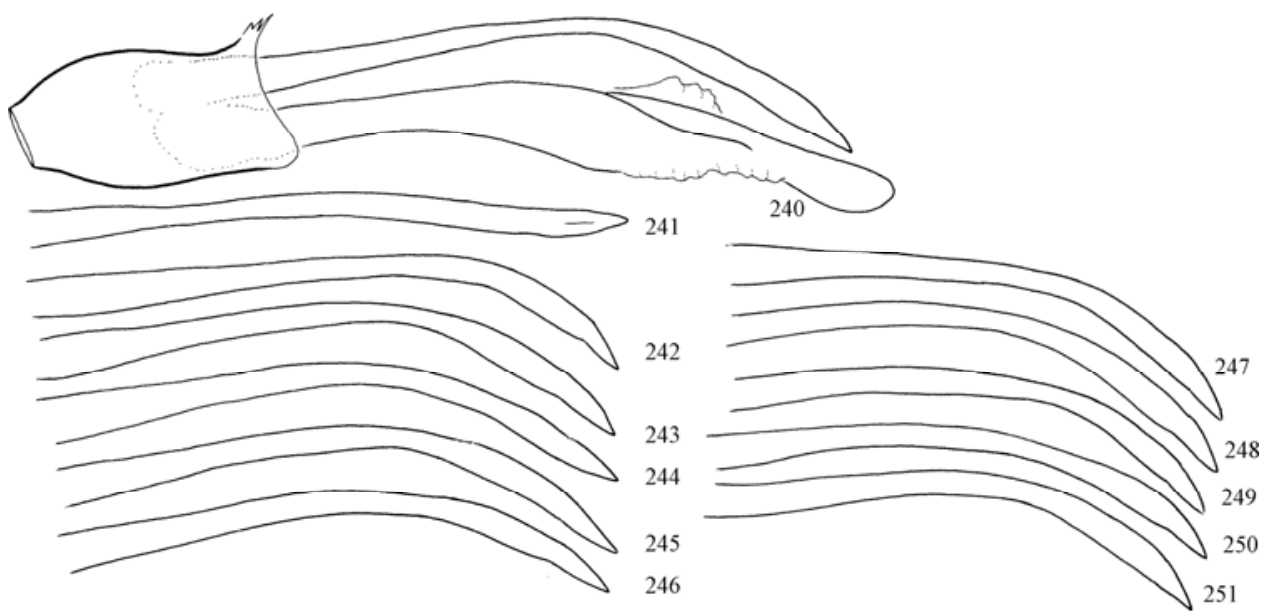

Figures 240-251. Melampophylax triangulifera Botosaneanu, 1957. $240=$ phallic organ in lateral view, $241=$ left paramere in dorsal view, 242-246 = left parameres from Hargitha Mts. Romania in lateral view, 247-251 = left parameres from Gurghiu Mts. Romania in lateral view. 


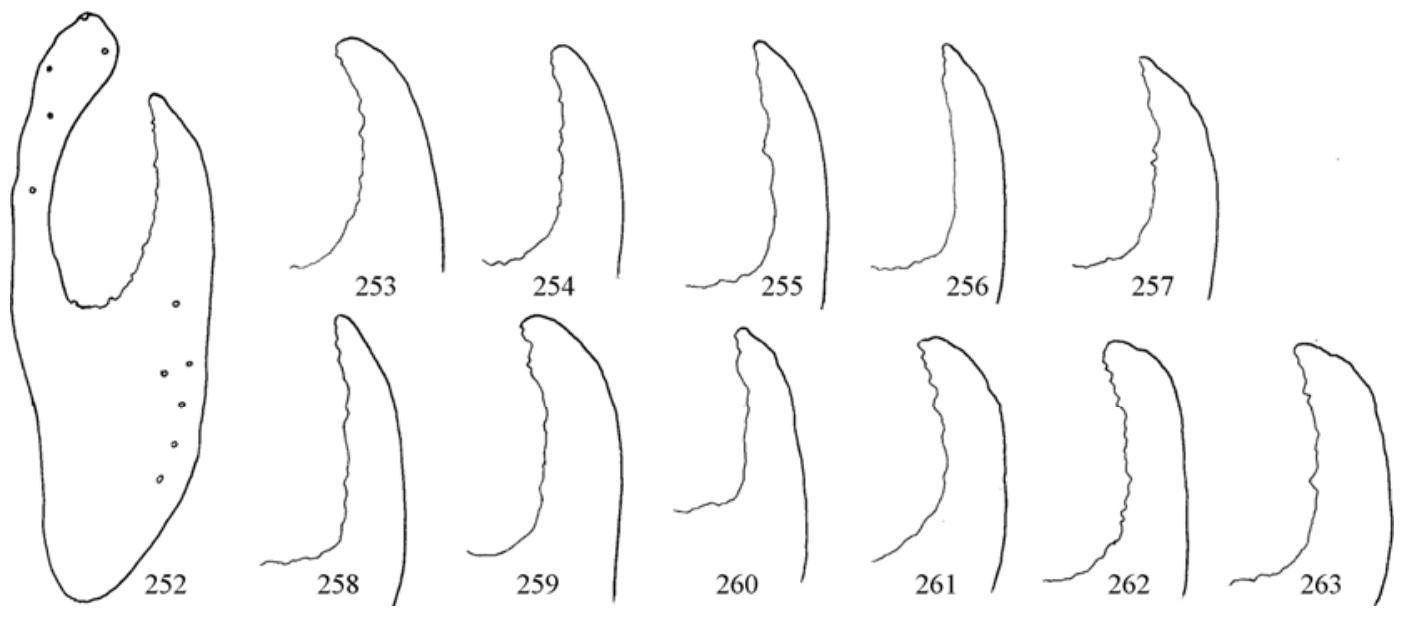

Figures 252-263. Melampophylax triangulifera Botosaneanu, 1957. 252 = left gonopods with mesal elongation from Hargitha Mts. Romania in lateral view, 253-257 = mesal elongation of gonopod from Hargitha Mts. Romania in ventral view, $258-263=$ mesal elongation of gonopod from Gurghiu Mts. Romania in lateral view.

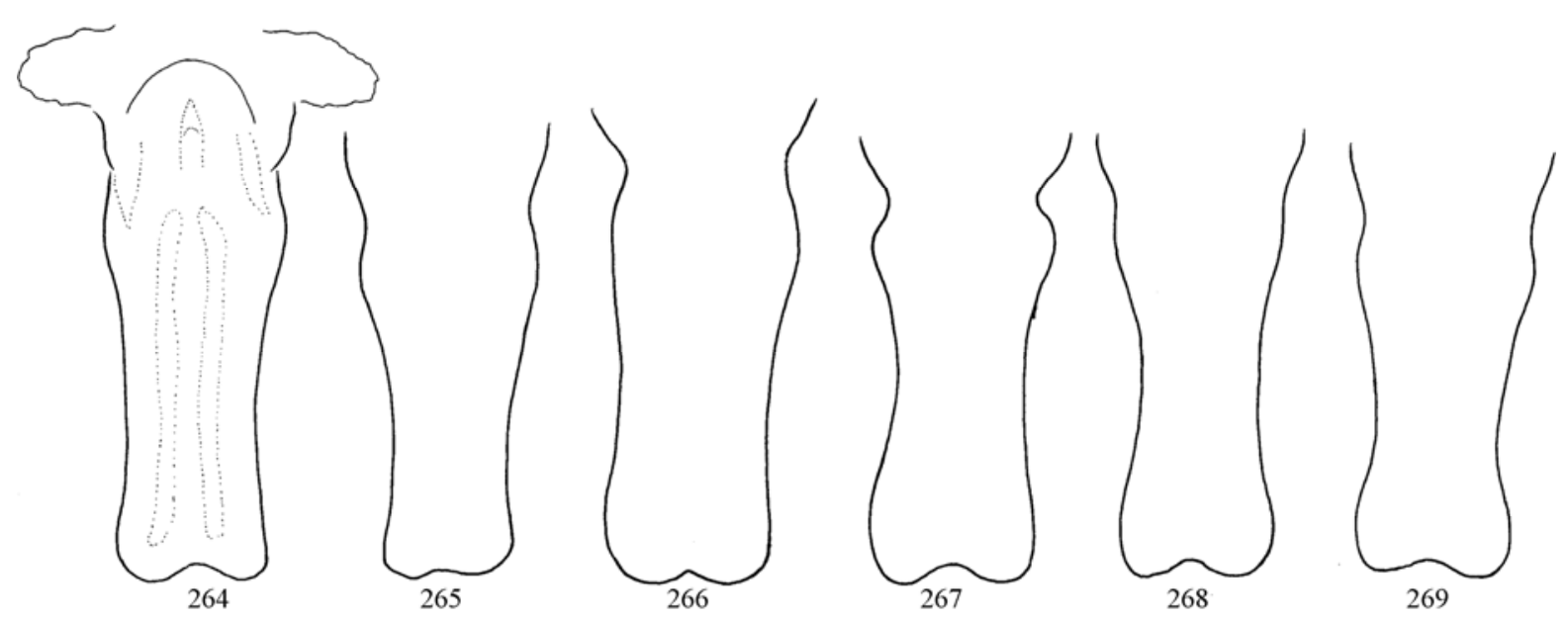

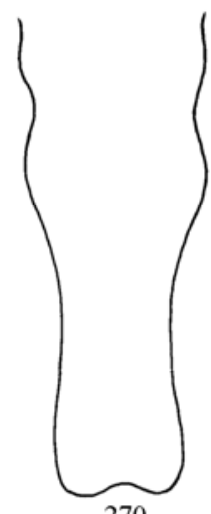

270

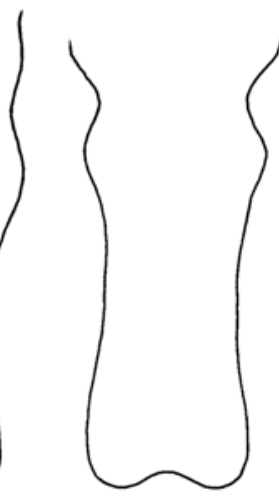

271

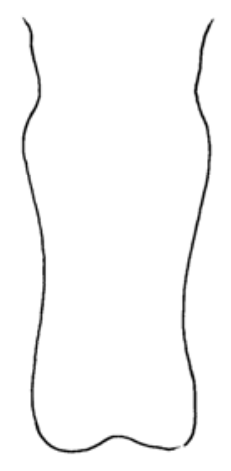

272

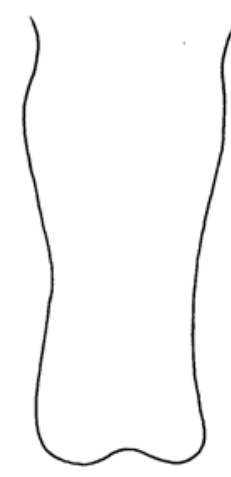

273

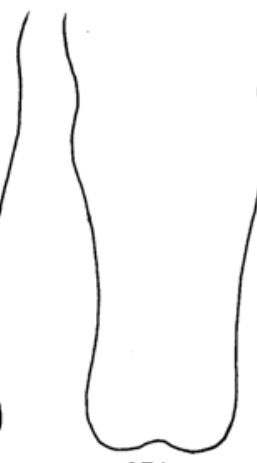

274

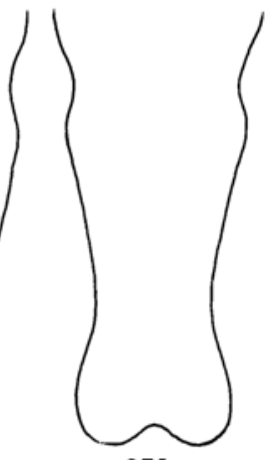

275

Figure 264-275. Melampophylax triangulifera Botosaneanu, 1957. 264-266 = dorsal vaginal sclerite complex from Kaliman Mts. Romania in dorsal view. 267-269 = dorsal vaginal sclerite complex from Hargitha Mts. Romania in dorsal view. 270-275 = dorsal vaginal sclerite complex from Gurghiu Mts. Romania in dorsal view. 
separating the two tribes, are fairly subtle and not very stable (Schmid 1998). Malicky (2001) examined the presumed differences in head, eye, and prothorax shape as well as in wing venation characters, and found them confused, not very useful. Even the most consistent character, the curvature of $\mathrm{M}$ veins on hindwing also varies in both tribes. $\mathrm{He}$ has concluded that no character has been found yet that clearly separates these two tribes and the genital structures remained alone to establish relationships.

We suggest that the paramere, the speciation phenotype discovered also in Rhadicoleptus genus, offers us a simple and stable character to separate Limnephilini from Stenophylacini. Timing of divergences is difficult especially for old, completely reproductively isolated lineages. One possibility is to compare genealogies of speciation genes/traits to genealogies of genes not involved in speciation process, such as the unlinked neutral loci. Genes/traits affecting reproductive isolation flow slow compared to neutral loci. Therefore speciation genes reflect species boundaries whereas loci not involved in speciation might show little phylogenetic resolution (Nosil \& Schluter 2011). However, this relation works not only in contemporary divergences, that easy to detect, but also in older lineages. After a preliminary throughout examination of all the Limnephilini and Stenophylacini genera we were surprised to realize that all the genera in the tribe Limnephilini has very specially evolved branched or broadly elaborated paramere head, and tribe Stenophylacini has simple paramere head. The only deviation may occur in both tribes is the reduction or simplification by the Willinston's law. This law suggests a particular tend towards reduction, a possible evolutionary mechanism of paramere complexity. This is a mechanism for reduction, simplification, or specialization. The reduction in the number of structural parts increases complexity by various complementary qualities: anisomerism, unpaired structures, connection density, path length, cluster development (Oláh et al. 2014). We have found the diverged architecture of the branched broad versus simple paramere head consistent in both tribes composed of many genera. Therefore we transfer genus Rhadico- leptus having simple paramere head from tribe Limnephilini to tribe Stenophylacini, in spite of the presence of larval abdominal gills with 3-4 filaments (Waringer et al. 2011).

A more comprehensive morphological fine structure analysis of paramere in Limnephilinae would give us more phenotypic tool in alpha taxonomy. In our preliminary survey we found only a few anomalies based only on paramere development. Termophylax having simple paramere tip was misplaced in Limnephilini (Nimmo 1995). Hesperophylax, Psychoronia and Crenophylax genera were again misplaced in Limnephilini with unusual paramere of very short shaft with broomlike burst of strongly sclerotized, recurved spines. In a recent revision, applying complex phylogenetic analysis with morphological characters, our tribe delimitation with paramere evolution has been confirmed. These three genera have been removed from tribe Limnephilini and grouped into a new tribe Hesperophylacini (Vshivkova et al. 2007).

Botosaneanu \& Riedel (1965) have analysed the geographical variation of the Rhadicoleptus alpestris species and they have established four subspecies, the name-bearing nominotypical subspecies, and three new subspecies. In distinguishing subspecies they relied mostly upon female genitalia, especially on the supragenital plate. They have found the periphallic organ very variable both within and between populations. The very tip of the male gonopods was found also specific, diverse, and stable at least at three subspecies. Similarly to the general practice, prevailing at that time, the parameres were not examined at all. Therefore they were unable to distinguish the four subspecies by male characters. Male and female genital characters were combined to successfully distinguish subspecies. Female holotypes were designated for subspecies of $R$. a. sylvanocarpaticus and $R$. a. macedonicus.

We have detected the paramere as a speciation trait to distinguish incipient species also in this small genus. It seems more and more general that, under contemporary speciation processes in isolated high mountain habitats and in the peripatry of the ancestral species, many limnephilid taxa 
produced young peripatric incipient species. This contemporary divergence has been powered by sexual selection and progressed in late Pleistocene. The evolving taxa can be clearly distinguished by the shape of the paramere phenotype. Paramere evolution has fully confirmed the validity of the four taxa established and elaborated so laboriously with fairly subtle character combinations by Botosaneanu \& Riedel (1965). Our species delimitation by paramere divergent evolution was also confirmed by shape analysis of the gonopods tips. This strongly sclerotized structure with very diverse and specific apical profile may participate in the copulatory processes as an auxiliary titillating, stimulation or harming device supplementing the basic paramere function. Finally we have recorded species specific shape for female supragenital plates, as well as for the dorsal vaginal sclerite complex. Vaginal sclerite complex was not documented by drawings in all species. Here we diagnose the five incipient species briefly by shapes of the parameres, gonopod apical tip and supragenital plates.

\section{Rhadicoleptus alpestris (Kolenati, 1848)}

(Figures 276-312, 313-349, 350-355)

Stenophylax alpestris Kolenati, 1848: 66.

Rhadicoleptus alpestris (Kolenati, 1848): Wallengren 1891: 72-73: transfered to the newly created genus Rhadicoleptus.

Rhadicoleptus alpestris alpestris (Kolenati, 1848): Botosaneanu \& Riedel 1965: 546-547. Established as nominal subspecies.

Diagnosis. The ancestral species of the complex. The lateral profile of parameres is characterized by low (shallow) curvature, by subbasal constriction and by subapical dilatation. This profile is very stable at the examined 43 populations on the entire distributional area from France to Serbia through Austria, Czech Republic, Hungary, Italy, and Slovakia. The paramere of a single population from Albania (Prokletije Mts.), probably in the contact zone with $R$. macedonicus, has a mixed fine structure with or without subbasal constriction and subapical dilatation. Ventral profile of the gonopod apical head is characterized by a single pointed tip accompanied by highly varying pattern of small lobes. Female supragenital plate long and narrow triangular. Unlike paramere the ventral profile of the gonopods and the supragenital plate in the population of the Prokletije Mts. with contact zone is typical. This may suggest that paramere seems more sensitive and liable in reinforcement processes of the hybrid zone.

Material examined. Albania, Prokletije Mts. Above village Doberdol, flush around a smaller tarn below tarn Liqeni i Dashit, 2080 m, N42 $32.008^{\prime}$ E20 04.653' 9. VII. 2011, leg. Z. Barina, A. Kovács, G. Puskás \& B. Sárospataki (9 males, 5 females; HNHM). Austria, "Dr. P. Kempny/ Gutenstein, N.-Oe. / 17.5.900", Niederösterreich, Gutenstein, 17.V.1900, leg. P. Kempny (1 male, Klapálek collection: K385, NMPC). "Dr. P. Kempny/ Gutenstein, N.-Oe. / 17.5. 900" Niederösterreich, Gutenstein, 21.V.1900, leg. P. Kempny (Klapálek collection: K386, 1 female, NMPC). "Dr. P. Kempny / Gutenstein, N.-Oe. / 17.5.900" Niederösterreich, Gutenstein, 21.V.1900, leg. P. Kempny (Klapálek collection: K387 1 female, NMPC). "Dr. P. Kempny / Gutenstein, N.Oe. / 17.5.900" Niederösterreich, Gutenstein, 21.V.1900, leg. P. Kempny (Klapálek collection: K388, 1 female, NMPC). "Hohen/tauern // 22.viii.02" Steiermark, Hohentauern S of Trieben, 22.8.1902, leg. F. Klapálek (Klapálek collection: K389, 1 male, NMPC). Czech Republic, E. Bohemia, Zdarské vrchy hills, NPR Radostinské raselinisté reserve (at light), 14-18.VI.1996, leg. J. Sumpich (12 males, 12 females; OPC). E. Bohemia, Orlické hory Mts. peatbog near Kunstátská kaple, 10.VI.1997, leg. P. Chvojka (6 males, 4 females; OPC). N. Bohemia, Jizerské hory Mts. Mala klecová louka peatbog, 8.VI.2002, leg. F. Krampl (6 males, 7 females; OPC). N. Moravia, Králicky Snéznik Mts. peatbog between Susina Mt. and Cerna kupa Mt. 7.VII.2000, leg. P. Chvojka (5 males, 4 females; OPC). Bohemia sept. Decinská vrch. NP Ceske Svycarsko, Krinice Zadni Jetrichovice, 50 53'57'N 14 21'31'E, 250m, (MT) V.-VI. 2007, leg. M. Tryzna (8 males, OPC). France, Departement Vosges, Plainfaing, gazon du Faing, 1.VII.2009, leg. G. Coppa, (5 males, OPC). Departement Ardennes, Hargnies, Croix-Gillet, 15.VI.2000, leg. G. Coppa, (4 males, 7 females, OPC). Departement Ardennes, Hargnies, Croix-Gillet, 6.VI.2014, leg. G. Coppa, (4 females, OPC). Departement Haute-Savoie, Sixt-Fer-à-Cheval, réserve de Passy, ru tourbeux, 23.VI. 2009, leg. G. Coppa, (1 male, 1 female, OPC). Hungary, Zemplén Mts. István spring, 7.VI.1955, leg. Gozmány, (1 male, OPC). Zemplén Mts. Makkoshotyka, 27.V.1961, light, (1 male, OPC). Zemplén Mts. Kemence Valley, sphagnum bog, 23.V.1962, singled leg. J. Oláh (4 males, 3 females; OPC). Zemplén Mts. Telkibánya, VI. 1981, light trap (1 male, OPC). Zemplén Mts. Telkibánya, 25-30.V. 1982, light trap (1 male, OPC). Zemplén Mts. Lászlótanya, 10-20.VII. 1982, light trap (1 male, 1 female; OPC). Zemplén Mts. Lászlótanya, 20.VI.- 10.VII. 1983, light trap (3 males, 1 female; OPC). Zemplén Mts. Lászlótanya, V. 1983, light trap (4 males, OPC). Zemplén Mts. Lászlótanya, 1-10.VI. 1983, 


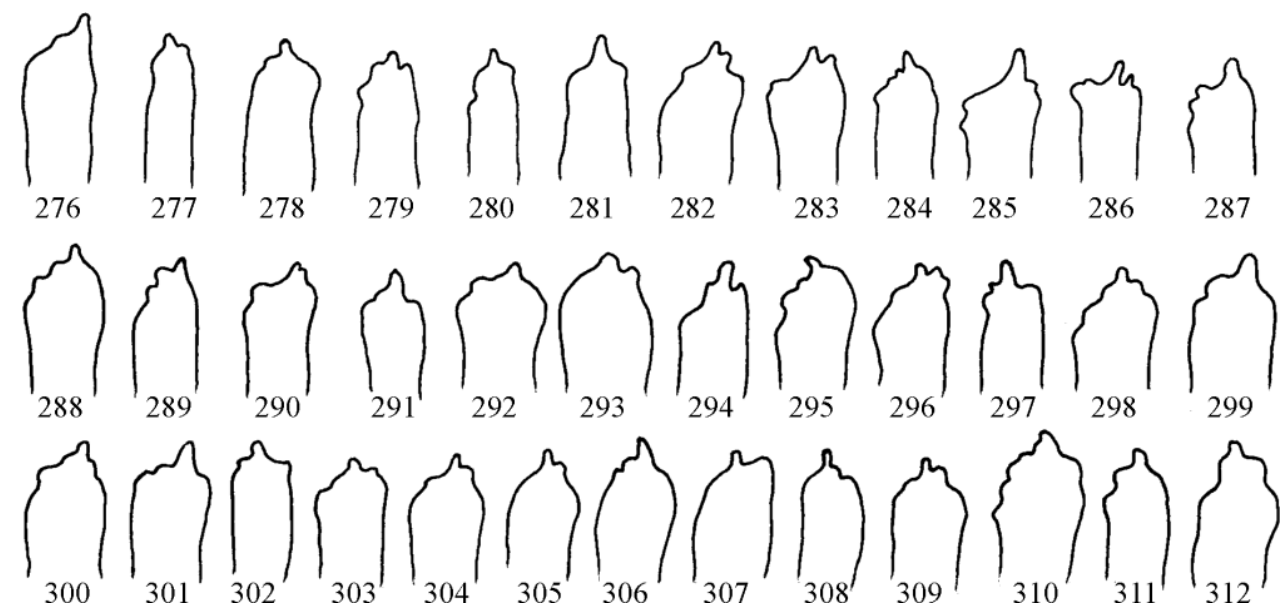

Figures 276-312. Rhadicoleptus alpestris (Kolenati, 1848). Apical tip of the gonopods in various populations in caudal view. 276-282 = Italy, 283-287 = France, 288-289 = Austria, 290-293 = Czech Republic, 295 = Slovakia, 296-298 = Hungary, Magyarszombatfa, 299-304 = Hungary, Zemplén Mts., 305-308 = Serbia, 309-312 = Albania, Prokletije Mts.
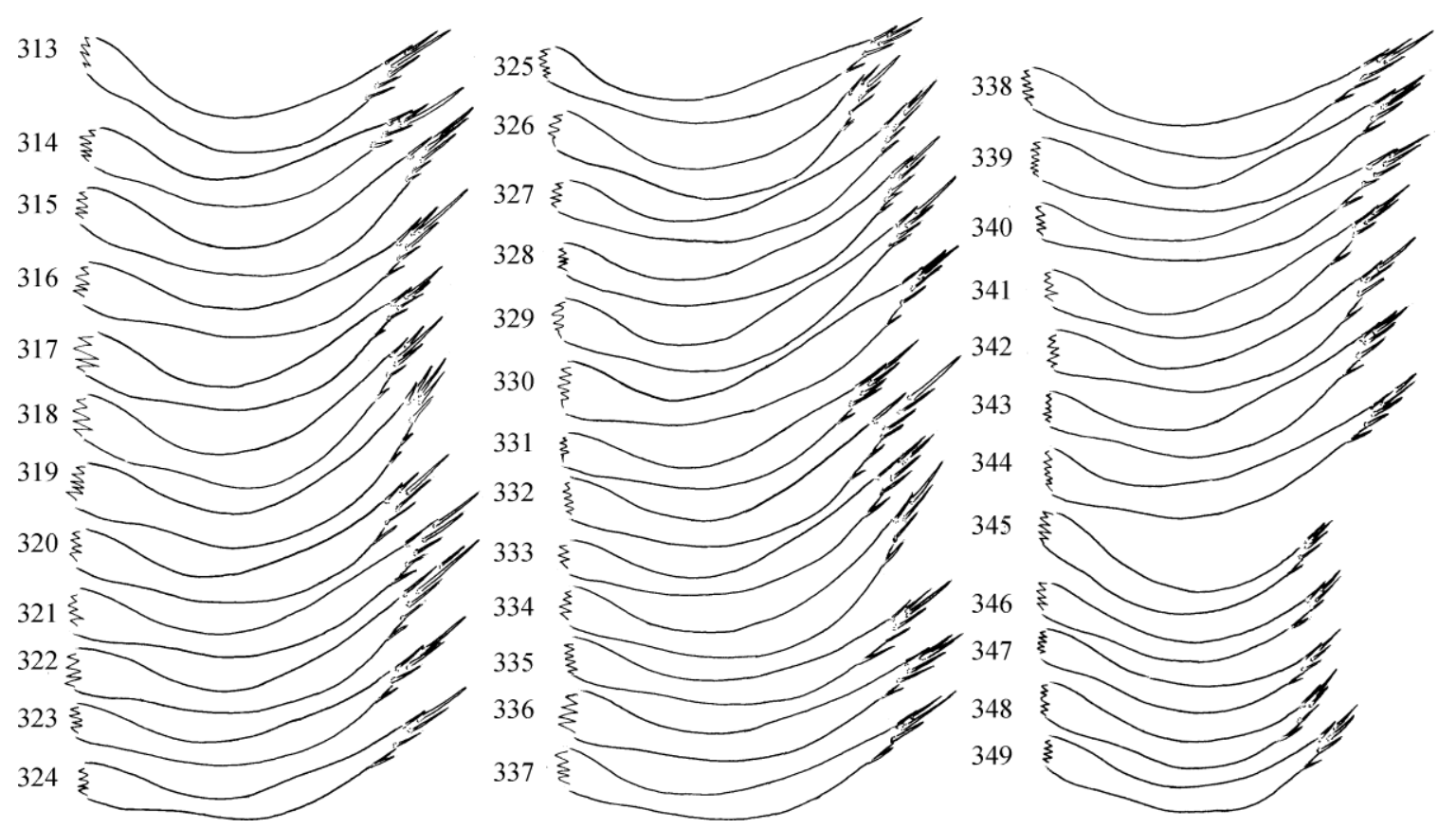

Figures 313-349. Rhadicoleptus alpestris (Kolenati, 1848). Left paramere in lateral view. 313-320 = Italy, 321-325 = France, 226-227 = Austria, 328-331 = Czech Republic, 332 = Slovakia, 333-335 = Hungary, Magyarszombatfa, 336-341 = Hungary, Zemplén Mts., 342-344 = Serbia, 345-349 = Albania, Prokletije Mts.

light trap (1 male, OPC). Zemplén Mts. Lászlótanya, 14.VI. 1985, light leg. Oláh (6 males, 8 females; OPC). Zemplén Mts. Lászlótanya, 1-10.V. 1986, light trap, (6 males, 9 females; OPC). Zemplén Mts. Lászlótanya, 10-20.V. 1986, light trap, (6 males, 9 females; OPC). Zemplén Mts. Lászlótanya, 20-30.V. 1986, light trap, (11 males, 21 females; OPC). Zemplén Mts. Füzér, Lászlótanya, 1-14.VII.1987, light trap, (1 male, OPC). Zemplén Mts. Telkibánya, 2530.V.1982, light trap, (1 male, OPC). Magyarszombatfa,
Szentgyörgyvölgyi stream, 18.V.1985, light trap (3 males, 5 females; OPC). Örség, Magyarszombatfa, Szentgyörgyvölgyi stream, 18.V.1985, light trap (3 males, 5 females; OPC). Örség, Magyarszombatfa, Szentgyörgyvölgyi stream, 13.V. 1985, light trap (1 male, 1 female; OPC). Örség, Magyarszombatfa, Szentgyörgyvölgyi stream, 3.V.1985, light trap (1 male, OPC). Örség, Magyarszombatfa, Szentgyörgyvölgyi stream, 21.V.1985, light trap (5 males, OPC). Örség, Magyarszombatfa, Szentgyörgyvölgyi stream, 14.V.1984, light 
trap (2 males, OPC). Szentpéterfölde, erdészház, 28.V.1988, light tap (2 males, OPC). Darány, borókás, Nagyberek, 18.V. 1988, leg. L. Ábrahám (1 male, OPC). Szőce, patakvölgy, 21.V.1984, leg. S. Nógrádi (4 males, 10 females; OPC). Szalafö, Pityerszer,1-15. VI. 1986, light trap (5 males, OPC). Italy, Lombardia, Carona (BG), torrente affl. Lago Rotondo, 2000m, 20.VII.2000 leg. M. Gaini \& O. Lodovici (30 males, 8 females, CNSMB; 9 males, 4 females, OPC). Lombardia, Branzi (BG), Rif. Laghi Gemelli, 2000m, 28.VII.1983, leg. CAI Bergamo (12 males, 5 females, CNSMB; 6 males, 1 female, OPC). Lombardia, Schilpario (BG), Malga Gaffione, 1957m, 8.VII.1995, leg. F. Albrici (9 males, 7 females, CNSMB). Lombardia, Valbondione (BG), Baite di Sasna, 1940m, 24.VII.1995, leg. F. Albrici (128 males, 15 females, CNSMB; 20 males, 1 female, OPC). Pejo (TN), Pian Venezia torrente, 2300m, 31.VII.2002, leg. O. Lodovici \& P. Pantini (1 male, CNSMB). Serbia, Zlatibor district, Zlatibor Mts, spring brook of Crni Rzav Stream beneath Mt. Cigota, N43 7.932', E1946.305', 1160 m, 25.V.2013, leg. P. Juhász, T. Kovács, G. Magos, G. Puskás, (14 males, 12 females; OPC). Zlatibor district, Zlatibor Mts, Crni Rzav Stream along the road No. 21, N4340.356', E1942.125', 1005 m, 14.05. 2014, T. Kovács, D. Murányi (4 males, 1 female; OPC). Slovakia, N Slovakia, Oravske Beskydy Mts. SE of Pilsko Mt. 1450m, 29.VI.1992, leg. P. Chvojka (1 male, NMPC).
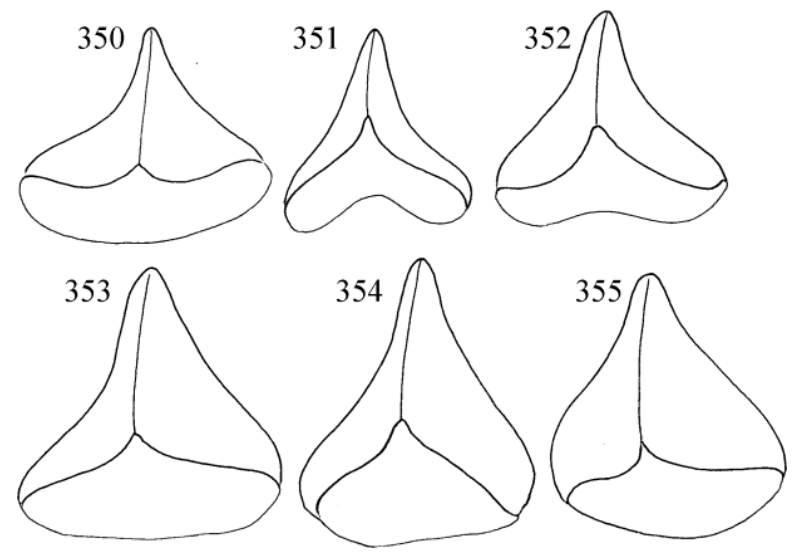

Figures 350-355. Rhadicoleptus alpestris (Kolenati, 1848). Supragenital plate of female genitalia in ventral view.

\section{Rhadicoleptus macedonicus Botosaneanu \& Riedel, 1965 stat. nov.}

(Figures 356-375,376-394, 395-398)

Rhadicoleptus alpestris macedonicus Botosaneanu \& Riedel, 1965: 550-551.

Diagnosis. The subspecies was established only by female character having supragenital plate very short similar to $R$. spinifer, but paramere and gonopod apical tip is different. The lateral profile of parameres is characterized by low (shallow) curvature, by narrowing gradually apicad without subbasal constriction and subapical dilatation. This profile is very stable in the examined populations of Bulgaria, Macedonia, and Albania. Ventral profile of the gonopod apical tip is without highly varying pattern of small lobes, a single blunt but varying lobe is present usually mesad. Female supragenital plate very short triangular, but as rounded as the supragenital plate of R.spinifer.

Material examined. Albania, Erseke County, Grammos Mts, mountaine pasture on the slope of Mt Varibob, $2.1 \mathrm{~km}$ NW of Mt Oukapeci, $2249 \mathrm{~m}, \mathrm{~N} 40.366220^{\circ}$ E20.770510 19.VII.2006, leg. Z. Barina, T. Pifkó \& D. Pifkó (4 males, 3 females, HNHM). Librazhd county, Jablanica Mts, Quarishte, brook $6.1 \mathrm{~km} \mathrm{E}$ of the village, $1899 \mathrm{~m}, \mathrm{~N} 41.24569^{\circ}$ E20.51238 4.VII.2008, leg. Z. Barina, T. Pifkó \& D. Pifkó (3 males, HNHM). Bulqizë district, Çermenikë Mts, brooks in open forest beneath Mt. Kaptinë, N41 ${ }^{\circ} 23.199^{\prime}$, E20 17.338', 1600 m, 21.06.2012, leg. Z. Fehér, T. Kovács, D. Murányi ( 3 males, 3 females; OPC). Bulgaria, Rila Mts, Jazovir Belmeken, springbrook, N42 ${ }^{\circ} 10^{\prime} 22.7^{\prime \prime}$, E23 ${ }^{\circ} 48^{\prime}$ 04.4”, 1332m, 23. VI. 2011, P. Juhász, T. Kovács, \& L. Urbán, (4 males, 1 female, OPC; 2 males, CSNMB). Macedonia, Korab Mts., Malá Korab Vrata, 20.VII.1930 leg Komarek (3 males, NMPC; 2 males, OPC).

\section{Rhadicoleptus meridiocarpaticus Botosaneanu \& Riedel, 1965 stat. nov.}

(Figures 399-404)

Rhadicoleptus alpestris meridiocarpaticus Botosaneanu \& Riedel, 1965: 549-550. Distinguishable only by female.

Diagnosis. The lateral profile of parameres is characterized by high (deep) curvature, even, uniform shape without narrowing, constriction and dilatation. Ventral profile of the gonopod apical tip is slightly dilated with subtle tiny irregular pattern. Female supragenital plate is long triangular, but more robust than at R.alpestris.

Material examined. Romania, Apuseni Mts. Vartop, spring streams, $\mathrm{N} 46^{\circ} 31.045^{\prime}$ E22 $2^{\circ} 39.821^{\prime}, 1209 \mathrm{~m}, 29 . \mathrm{V}$. 2013, singled leg. J. Oláh, E. Bajka, Cs. Balogh, \& G. Borics (1 female, OPC). Apuseni Mts. Vartop, spring stream (Flescula), N46 ${ }^{\circ} 31$ '07,23" E22 ${ }^{\circ} 39^{\prime} 41,69 ” 1209 \mathrm{~m}, 14 . \mathrm{V}$. 2014, leg. Cs. Balogh \& B.V. Béres (1 male, OPC). 

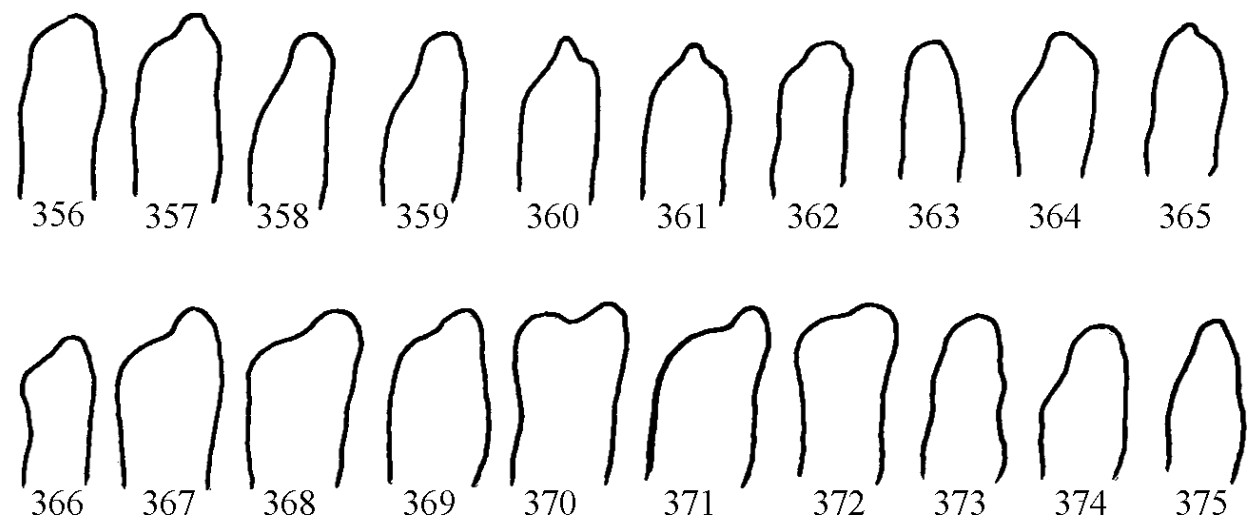

Figures 356-375. Rhadicoleptus macedonicus Botosaneanu \& Riedel, 1965. Apical tip of the gonopods in various populations in caudal view. 356-360 = Bulgaria, 361-365 = Macedonia, 366-368 = Albania, Cermenike Mts. 369-371 = Albania, Jablanica Mts., 372-375 = Albania, Grammos Mts.

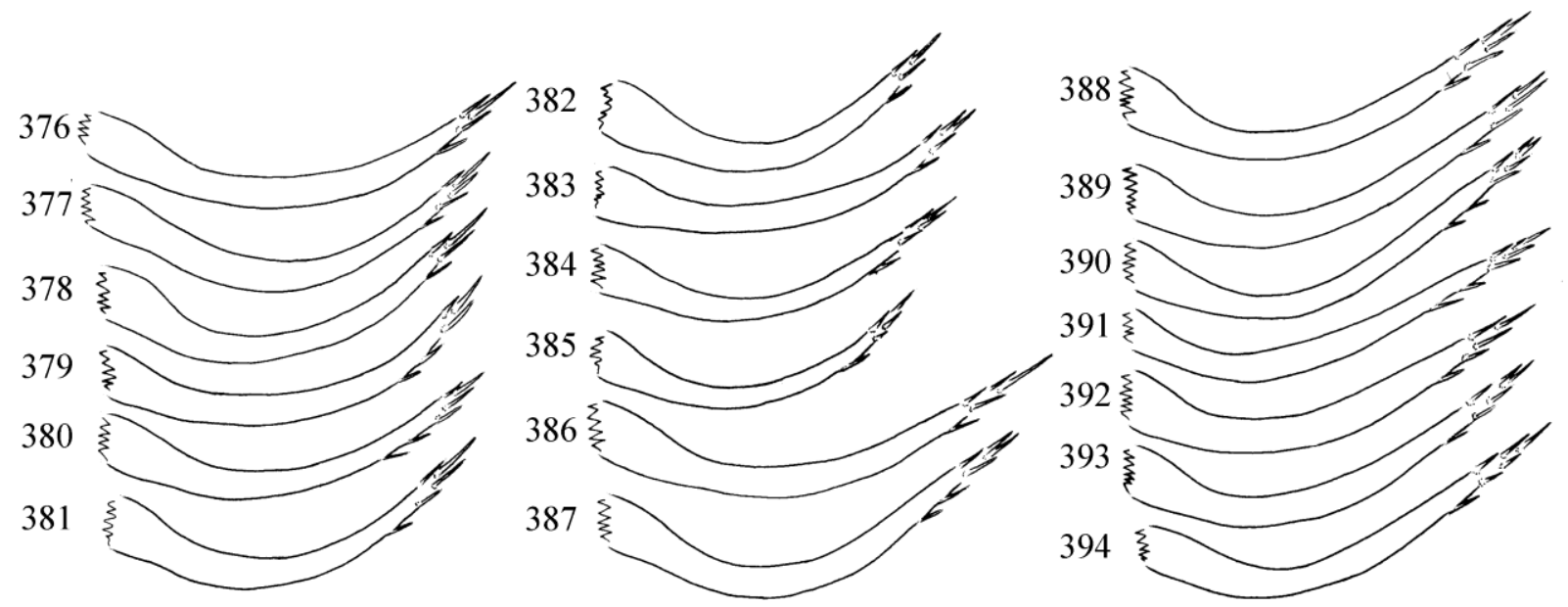

Figures 376-394. Rhadicoleptus macedonicus Botosaneanu \& Riedel, 1965. Left paramere in lateral view. 376-380= Bulgaria, 381-385 = Macedonia, 386-388 = Albania, Cermenike Mts. 389-391 = Albania, Jablanica Mts., 392-394 = Albania, Grammos Mts.

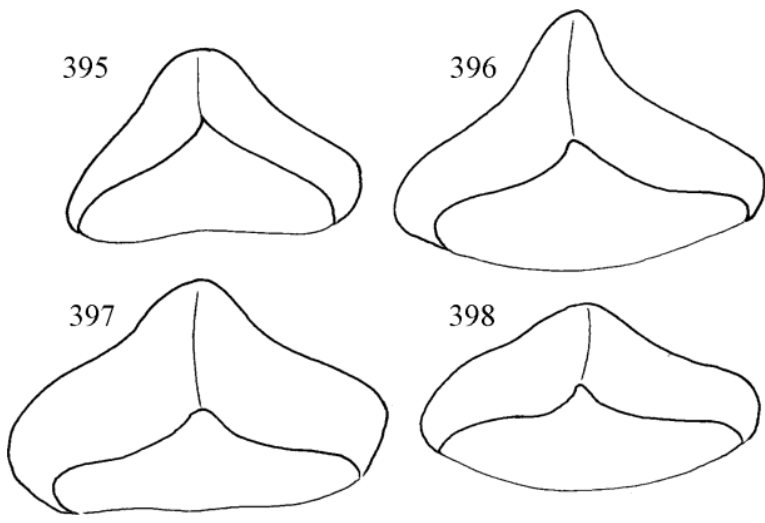

Figures 395-398. Rhadicoleptus macedonicus Botosaneanu $\&$ Riedel, 1965. Supragenital plate of female genitalia in ventral view.

\section{Rhadicoleptus spinifer (McLachlan, 1875) stat. restit.}

(Figures 405-412)

Stenophylax spinifer McLachlan, 1875: 120.

Rhadicoleptus alpestris spinifer (McLachlan, 1875): Malicky 1983: 185. Downgraded to subspecies.

Diagnosis. Paramere is slender. The lateral profile of parameres is characterized by very high (deep) curvature, uniform shape with some middle narrowing. Ventral profile of the gonopod apical tip is with a long needle-shape point. Female supragenital plate is very low rounded, the dorsal profile of the vaginal sclerite complex without middle constriction. 
Material examined. France: Departement Lozère, Le Pont-de-Montvert, source tourbeuse chemin Hospitalet vers Pont-du-Tarn, 26.VI.2014, leg. G. Coppa (3 males, 3 females; OPC). Lozère Departement, Nasbinals, lac des Salhiens, 29. VI. 2010, leg. G. Coppa (3 males, 1 female; OPC). Departement Hautes-Pyrénées, Vielle-Aure, Neste d'Aure, en amont du barrage, 10. VII. 2009, leg. G. Coppa (4 males, 4 females; OPC). Departement Puy-de-Dôme, MontDore, ru prairie tourbeuse, NW Puy Marellh, 25. V. 2009, leg. G. Coppa (4 males, 2 females; OPC). Departement Hautes-Pyrénées, Gavarnie, zone humide près du barrage d'Ossus, petit plateau, 27. VII. 2010, leg. G. Coppa (4 males, 3 females; 2 males, 2 females; OPC; CSNMB). Departement Hautes-Pyrénées, Cauterets, source près du restaurant lac de Gaube, 18. VII. 2010, leg. G. Coppa (2 males, 2 females; OPC)..

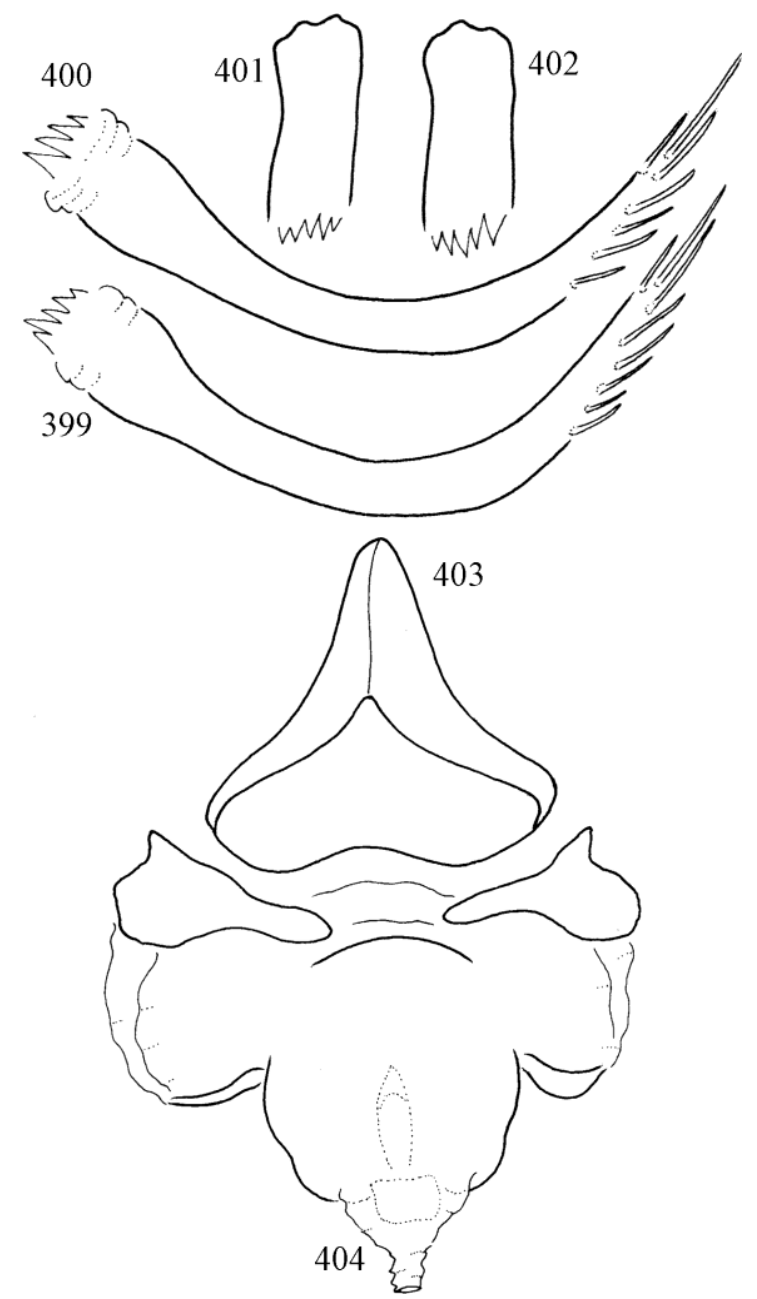

Figures 399-404. Rhadicoleptus meridiocarpaticus Botosaneanu \& Riedel, 1965. 399-400 = left paramere in lateral view. 401-402 = apical tip of the gonopods in caudal view, $403=$ supragenital plate of female genitalia in ventral view, $404=$ dorsal vaginal sclerite complex in dorsal view.

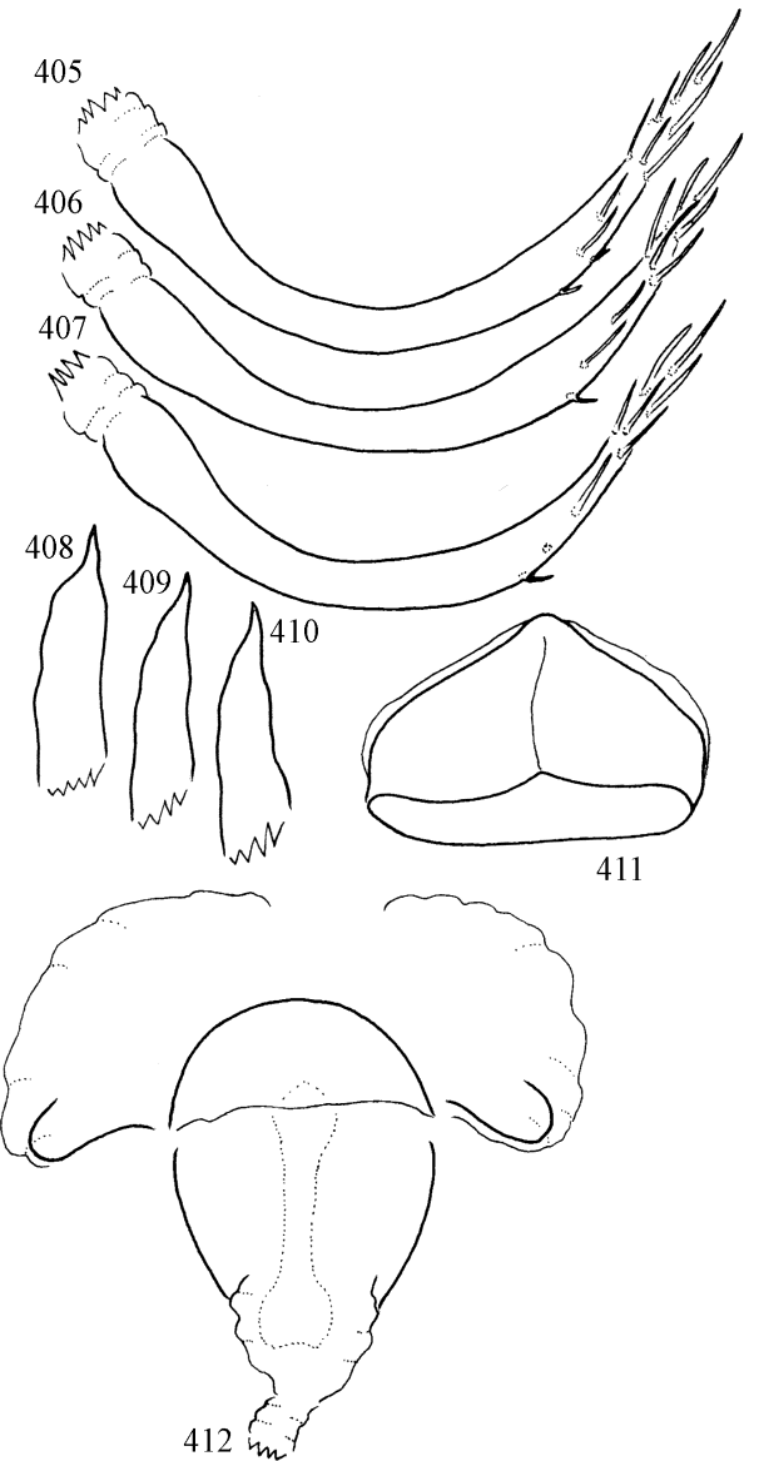

Figures 405-412. Rhadicoleptus spinifer (McLachlan, 1875). $405-407=$ left paramere in lateral view. $408-410=$ apical tip of the gonopods in caudal view, $411=$ supragenital plate of female genitalia in ventral view, $412=$ dorsal vaginal sclerite complex in dorsal view.

\section{Rhadicoleptus sylvanocarpaticus Botosaneanu \& Riedel, 1965 stat. nov.}

(Figures 413-422)

Rhadicoleptus alpestris sylvanocarpaticus Botosaneanu \& Riedel, 1965: 547-549. Distinguishable by both sexes.

Diagnosis. Paramere is very robust: The lateral profile of parameres is characterized by very short 
(very shallow) curvature, by uniform, even shape without narrowing, and constriction, but with some apical dilatation. Ventral profile of the gonopod apical tip is with a single blunt narrowing. Female supragenital plate is very long triangular, the dorsal profile of the vaginal sclerite complex with slight middle constriction.

Material examined. Romania, Muntii Lapusului (Lápos),
Valeni (Mikolapatak), peat bog, its inflow and outflow

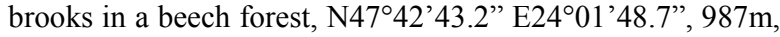
23.V.2006 leg. L. Dányi, M. Földvári, J. Kontschán \& D. Murányi (1 male NHMB). Ukraine, Zakarpatia province, Mizhhirya raion, Kolochava (Alsókalocsa), right sidebrook of Tereblja River by the village, N48 $25.41^{\prime}$ E23 ${ }^{\circ} 41.56^{\prime}$, $565 \mathrm{~m}, 16.05 .2002$, leg. D. Murányi (2 males, 4 females, HNHM). Tiachiv raion, Krasna Mts, beech forest edge in the upper valley of Luzanka River, N48 $22.564^{\prime}$ E23 ${ }^{\circ} 45.081^{\prime}$, 1295m, 19.05.2002, leg. D. Murányi (1 female, HNHM).

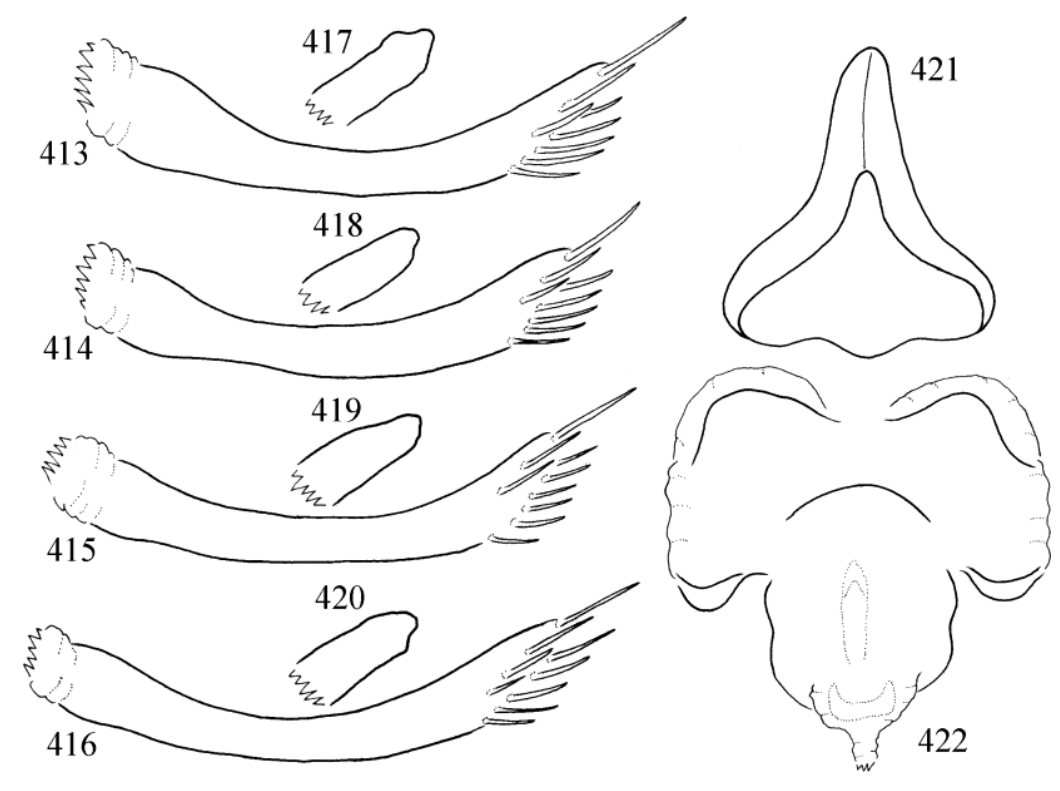

Figures 413-422. Rhadicoleptus sylvanocarpaticus Botosaneanu \& Riedel, 1965. 413-416 = left paramere in lateral view. $417-420=$ apical tip of the gonopods in caudal view, $421=$ supragenital plate of female genitalia in ventral view, $422=$ dorsal vaginal sclerite complex in dorsal view .

\section{Tribe Chaetopterygini Hagen, 1858}

\section{Genus Annitella Klapálek, 1907}

Type species: Annitella kosciuszkii Klapálek, 1907 (by subsequent designation of Schmid 1952b)

The genus Annitella, similarly to the small Chaetopterygopsis genus, has lost the paramere on the phallic organ. This important speciation trait has essential function in sexual selection processes; its divergence contributes as an effective barrier to reproductive isolation. The loss of such an important structure and its function or its replacement or its compensatory change by other structure might be associated with increased complexity by gene duplication. Gene duplication is known as a primary source of genetic material available for evolution of genes with new functions or with acquiring unique functionality by mutation both on coding and regulatory region, or by subdividing their ancestor's function (Taylor \& Raes 2004). Regulatory mutations in elements of duplicated genes could also produce novel gene expression patterns significantly altering morphological development (Ohta 2003).

In genus Annitella the reproductive barrier function of the lost paramere, as an adaptive nonneutral speciation trait, is replaced by the paraproct. This speciation trait is particularly modified in this genus; large surface contact has been evolved to exert reproductive isolation functions during any of the possible mating or copulatory processes: sensory manipulation in mating, sperm removal in sperm competition, fertilization cont- 
rol in sexual conflict, post-copulatory sperm selection in cryptic female choice. Its adaptive nature is consistently indicated by its stability as we have demonstrated by its diverged trait matrices.

\section{Annitella kosciuszkii new species complex}

Difficulties in species delimitation in this species complex have been reported and well documented by phenotypic polymorphism (Botosaneanu 1973) and by high variability due to newly formed hybrids (Szczęsny 1979). However, both polymorphism and hybrid variability were established mostly on neutral traits, namely on the posterior processes of tergite VIII. That time the neutral or nearly neutral theories of molecular evolution were just formulated. Taxonomists have not distinguished between neutralism and selectionism. The estimation of synonymous and nonsynonymous substitution rate was just on the beginning.

Taxonomy was relied mostly upon indiscriminate shape analysis. In several species of the genus Annitella the unique posterior processes of tergite VIII are extremely variable both inside and between populations. This neutral trait is highly exposed to various stochastic processes: mutation, genetic drift, effective population size, migration, effective gene flow, recombination, and reinforcement.

We have revised this small species complex by the speciation trait of the paraproct and found species delimitation rather clear. The paraproct together with the slender digitate cerci and the segment $X$ forms the superanal genitalic complex. The segment $\mathrm{X}$ itself is reduced to hold the paraproct and cerci fusing together into this very spectacular superanal complex superimposed and sheltered dorsally by the enlarged tergit VIII with its variable posterior processes. The paraproct itself is a basally fused pair of rather complex bilobed structure composed of lateral and mesal lobes. The species are best distinguished by the shape and development of the lateral and mesal lobes and the species specific differences are best demonstrated in its ventral view. Intrapopulational and infrapopulational variation of the paraproct is demonstrated in diverged trait matrices in lateral view. There are individual variations, any specimens have its identity, like every human has its own ear-lobe or fingerprint identity, but the paraproct architecture of each species is very stable.

The function of the lost paramere may be partially covered by the bifid sclerotized tip of the aedeagus. However its apparent stability is practically unreliable in routine taxonomic studies due to its very small size and its shape and position dependence on the erection state of the aedeagus. The state of endophallus retraction or protrusion may highly modify the visibility and the shape variation of the bifid apices of the aedeagus. We have found rather consistent stability in the shape specificity of the female anal tube in dorsal view. Published historical drawings contradicting each others are prepared from dry, uncleared specimens and the protruded and copulatory state of various external substructures is very variable that modify significantly each drawings. Especially the shape of supragenital plate is very sensitive to copulatory influences.

We have examined newly collected materials as well as the historical materials deposited in the Klapálek's Collection in NMPC, Prague, Czech Republic and in the Dziędzielewicz's collection in SMNHL, Lviv, Ukraine.

\section{Annitella chomiacensis (Dziędzielewicz, 1908)}

(Figures 423-431, 434, 438-439, 452-453)

Heliconis chomiacensis Dziędzielewicz, 1908: in Dziędzielewicz \& Klapálek 1908a: 22-23, Dziędzielewicz \& Klapálek 1908b: 250-255

Annitella chomiacensis (Dziędzielewicz, 1908): Raciecka 1934: 240-241, Heliconis synonymised with Annitella. A. chomiacensis redescribed and redrawn.

Annitella chomiacensis chomiacensis (Dziędzielewicz, 1908): Szczęsny 1979: 260, reduced to subspecies status based on supposed crossing between A. chomiacensis and A. lateroproducta.

Annitella chomiacensis (Dziędzielewicz, 1908): Botosaneanu 1995: 82, reinstate the species status.

Diagnosis. The lateral lobe of the paraproct is shorter than the mesal lobe, and the mesal lobe is very robust as visible both in lateral and ventral view. The sclerotized tip of the aedeagus is most 

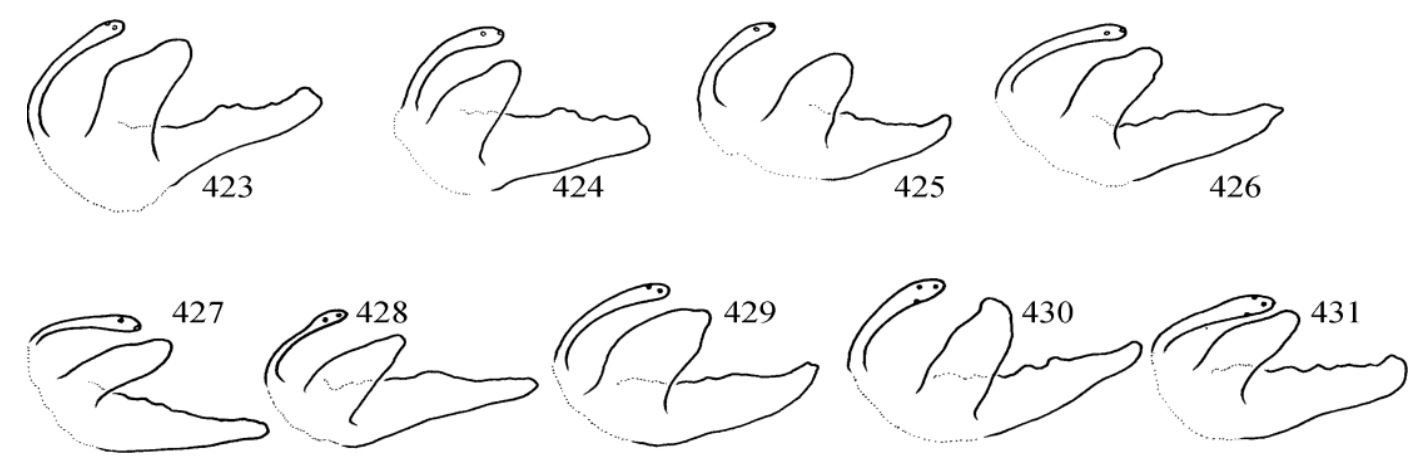

Figures 423-431. Annitella chomiacensis (Dziedzielewicz, 1908). Superanal genitalic complex in lateral view.
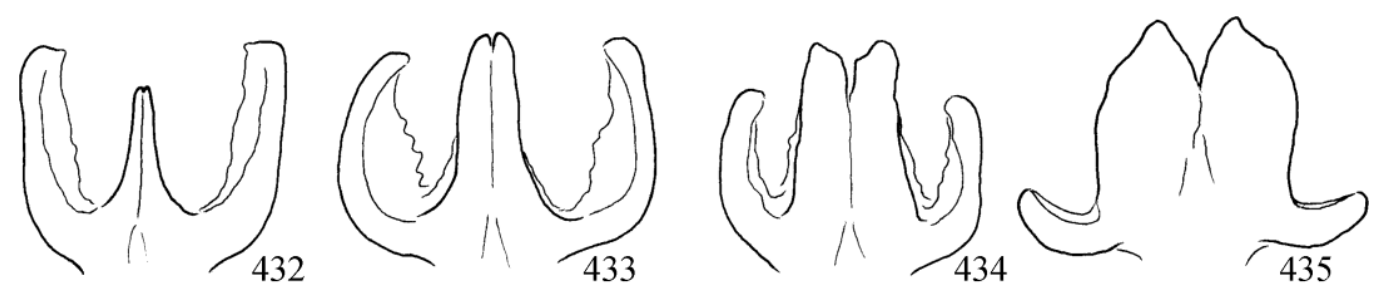

Figures 432-435. Superanal genitalic complex in ventral view. $432=$ A. lateroproducta, $433=$ A. kosciuszkii, $434=A$. chomiacensis, $435=$ A. wolosatka sp. nov.

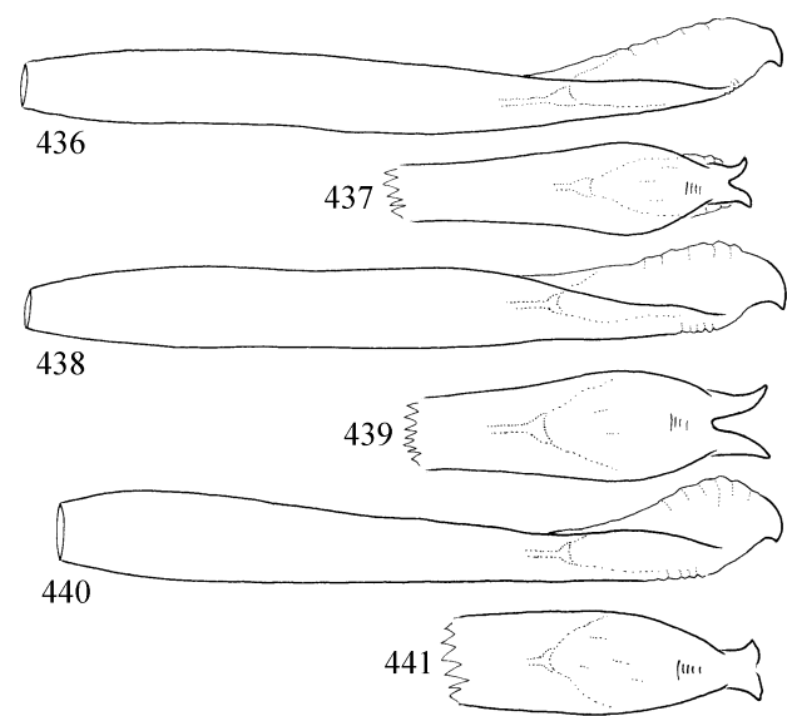

Figures 436-441. Aedeagus in lateral and ventral view. 436-437 = A. kosciuszkii, 438-439= chomiacensis 440-441 = A. wolosatka sp. $\mathrm{n}$.

developed and diverted laterad. Female anal tube with wide mesal excision in dorsal view, lateral apodemes of the vaginal sclerite complex slender, and laterad directed. The locus typicus of this spe cies is on the southern slope of the Khomiak Mts in Gorgany, Ukraine.

Material examined. Ukraine, original label: "Chomiak, Błotek, 14.X.1907, leg. J. Dziędzielewicz” (1 male, Klapálek's Collection in NMPC: K383). Ukraine: original label: "Chomiak, Błotek, 15.X.1907, leg. J. Dziędzielewicz" (1 male, Klapálek's Collection in NMPC: No. 48). Ukraine: original label: "Chomiak, Blotek, 16.X.1907, leg. J. Dziedzielewicz" (1 female, Klapalek's Collection in NMPC: No. 41). Ukraine: original label: "Chomiak, Błotek, X.1907” (1 male, Dziedzielewicz's collection in SMNHL: BS.024, E24.12.14.01/09). Ukraine: original label: "Czarnohora, Kozmieska, 16.X.1908" (1 male, Dziędzielewicz's collection in SMNHL: BS.012). Ukraine: original label: "Chomiak, Błotek, 15.X.1907" (1 female, Dziędzielewicz's collection in SMNHL: BS.029, E24.12.14.01/04). Ukraine: original label: "Tatarow, (Prutec), 7.X.1905" (1 male, Dziędzielewicz icz's collection in SMNHL: BS.004). Ukraine: original label: "Chomiak, Potok Barani, 16.X.1907" (1 male, Dziędzielewicz's collection in SMNHL: BS.028, E24.12.14.01/06). Ukraine: original label: "Chomiak, Potok Barani, 16.X.1907" (1 male, Dziedzielewicz's collection in SMNHL: BS.022, E24.12.14.01/05). Ukraine: original label: "Chomiak, Blotek, 6.X.1907" (1 male, Dziędzielewicz's collection in SMNHL: BS.025, E24.12.14.01/01). Ukraine: original label: "Chomiak, Błotek, 4.X.1907” (1 male, Dziędzielewicz's collection in SMNHL: BS.023, E24.12.14.01/03). 


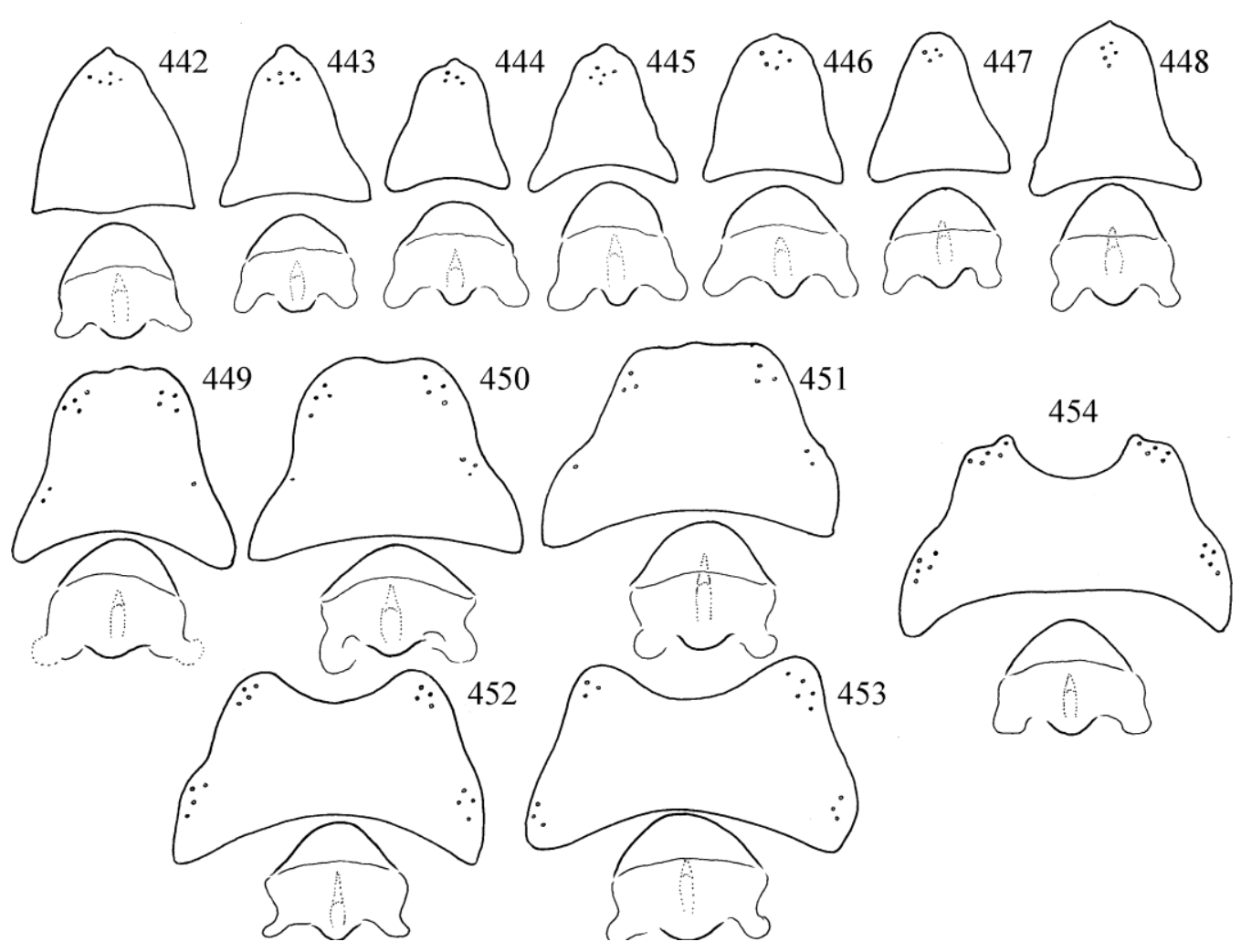

Figures 442-454. Female anal tube (fused tergit IX and X) and the dorsal vaginal sclerite complex in dorsal view. 442-448 = A. lateroproducta, 449-451 = A. kosciuszkii, 452-453 = chomiacensis, $454=$ A. wolosatka sp. nov.

Annitella kosciuszkii Klapálek, 1907 stat. restit.

(Figures 433, 436-437, 449-451, 455-470)

Annitella kosciuszkii Klapálek, 1907: 30-31.

Annitella kosciuszkii Klapálek, 1907: Dziędzielewicz 1911: 46-47, female description.

Annitella kosciuszkii Klapálek, 1907: Racięcka 1934: 241243, redescribed and redrawn.

Annitella kosciuszkii Klapálek, 1907: Szczęsny 1979: 260, reduced to a hybrid status formed in hybride zone by Annitella lateroproducta $\mathrm{x}$ Annitella chomiacensis.

Annitella kosciuszkii Klapálek, 1907: Malicky 2005: 572, a hybrid status formed in hybride zone by Annitella lateroproducta $\mathrm{x}$ Annitella chomiacensis reconfirmed.

Annitella dziedzielewiczi Schmid, 1952b: 157-158. Schmid distinguished A. dziedzielewiczi from A. kosciuszki mostly or even exclusively by the shape of the posterior process of tergite VIII.

Annitella dziedzielewiczi Schmid, 1952: Szczęsny 1979: 260, reduced to a hybrid status formed in hybride zone by Annitella lateroproducta $\mathrm{x}$ Annitella chomiacensis.

Annitella dziedzielewiczi Schmid, 1952: Malicky 2005: 572, hybrid status formed in hybride zone by Annitella lateroproducta x Annitella chomiacensis reconfirmed.

Annitella transylvanica Murgoci, 1957 in Murgoci \& Botosaneanu 1957: 139-1942.
Annitella transylvanica Murgoci, 1957: Botosaneanu 1973: 132-134: female described, species status reconfirmed.

Annitella transylvanica Murgoci, 1957: Szczęsny 1979: 260: reduced to a hybrid status formed in hybride zone by Annitella lateroproducta $\mathrm{x}$ Annitella chomiacensis.

Annitella transylvanica Murgoci, 1957: Botosaneanu 1995: 82: species status resurrected.

Annitella transylvanica Murgoci, 1957: Malicky 2005: 572: hybrid status formed in hybride zone by Annitella lateroproducta x Annitella chomiacensis reconfirmed.

Annitella dziedzielewiczi Schmid, 1952. A synonym of A. kosciuszki. syn. nov.

Annitella transylvanica Murgoci, 1957. A synonym of A. kosciuszki. syn. nov.

Diagnosis. The lateral lobe of the paraproct has almost equal length with the mesal lobe, and the mesal lobe is slender as visible both in lateral and ventral view. The sclerotized tip of the aedeagus has some flat apical surface. Female anal tube without wide mesal excision in dorsal view, lateral apodemes of the vaginal sclerite complex rounded. The locus typicus of this species is in the Czarnohora Mts. Ukraine. 
Material examined. Romania, Maramures county, Rodna Mts. Borsa-Statiunea Borsa, stream along the road towards Prislop Pass, 1014 m, N47 $37^{\circ}$ ' 34.0' 'E24 49' 13.0', 26.IX. 2006 leg. Dányi, J. Kontschan D. Murányi, (1ठ HNHM). Romania, Rodna Mts. small spring streamlets on the Bistrita

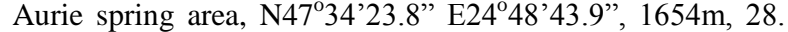
IX. 2014, leg. J. Oláh \& Cs. Balogh (1 male, OPC). Rodna Mts. Complex Borsa, small side spring stream of Fantana Stream, 29. IX. 2014, leg. J. Oláh \& Cs. Balogh (1 male, 1 female; OPC). Ukraine, original label: "Chomiak, Błotek, 13.X.1907, leśnicz, leg. J. Dziedzielewicz” (1 male, Klapálek's Collection in NMPC: No. 14). Ukraine: original label: "Worochta, Okolice, 9.X.1908, leg. J. Dziędzielewicz (1 male, Klapálek's Collection in NMPC: No. 13). Ukraine: original label: "Worochta, Okolice, 23.X.1908, leg. J. Dziędzielewicz" (1 female, Klapálek's Collection in NMPC: No. 16). Ukraine: original label: "Worochta, Okolice, 24.X.1908, leg. J. Dziędzielewicz" (1 male, Klapálek’s Collection in NMPC: K 384). Ukraine: original label: "Worochta, Okolice, 26.X.1908, leg. J. Dziędzielewicz" (1 male, Klapálek's Collection in NMPC: No. 15). Ukraine: original label: "Worochta, Okolice, 12.X.1908" (1 male, Dziędzielewicz's collection in SMNHL: No. 1240). Ukraine: original label: "Worochta, Okolice, 14.X.1908" (1 male, Dziędzielewicz's collection in SMNHL: No. 1238). Ukraine: original label: "Czarnohora, Kozmieska, 12.X.1908" (1 male, Dzię- dzielewicz's collection in SMNHL: No. 1250). Ukraine: original label: "Czarnohora, Foreszczynka, 8.X.1910" (1 male, Dziędzielewicz's collection in SMNHL: No. 1248). Ukraine: original label: "Worochta, 5.X.1909" (1 male, Dziedzielewicz's collection in SMNHL: No. 1246). Ukraine: original label: "Worochta, 9.X.1910" (1 male, Dziędzielewicz's collection in SMNHL: No. 1247). Ukraine: original label: "Worochta, Okolice, 5-11.X.1908" (1 male, Dziędzielewicz's collection in SMNHL: No. 1246). Ukraine: original label: "Worochta, 22.X.1909" (1 female, Dziędzielewicz's collection in SMNHL: No. 1242). Ukraine: original label: "Czarnohora, Zawojela, 2.X.1908” (1 female, Dziędzielewicz's collection in SMNHL: No. 1252). Ukraine: original label: "Worochta, 17.X.1909" (1 male, Dziędzielewicz's collection in SMNHL: No. 1253). Ukraine: original label: "Worochta, 22.X.1909" (1 male, Dziedzielewicz's collection in SMNHL: No. 1244). Ukraine: original label: "Tatarow, Blotek, X.1906" (1 male, Dziędzielewicz's collection in SMNHL: No. 1243). Ukraine: original label: "Czarnohora, Kozmieska, 17.X.1908" (1 male, Dziędzielewicz's collection in SMNHL: No. 1249). Ukraine: original label: "Czarnohora, Kozmieska, 13.X.1909" (1 male, Dziędzielewicz's collection in SMNHL: No. 1241). Ukraine: original label: "Worochta, Okolice, 8.X.1908" (1 male, Dziędzielewicz's collection in SMNHL: No. 1245). Ukraine: original label: "Worochta, 9.X.1910" (1 male, Dziędzielewicz's collection in SMNHL: No. 1255).
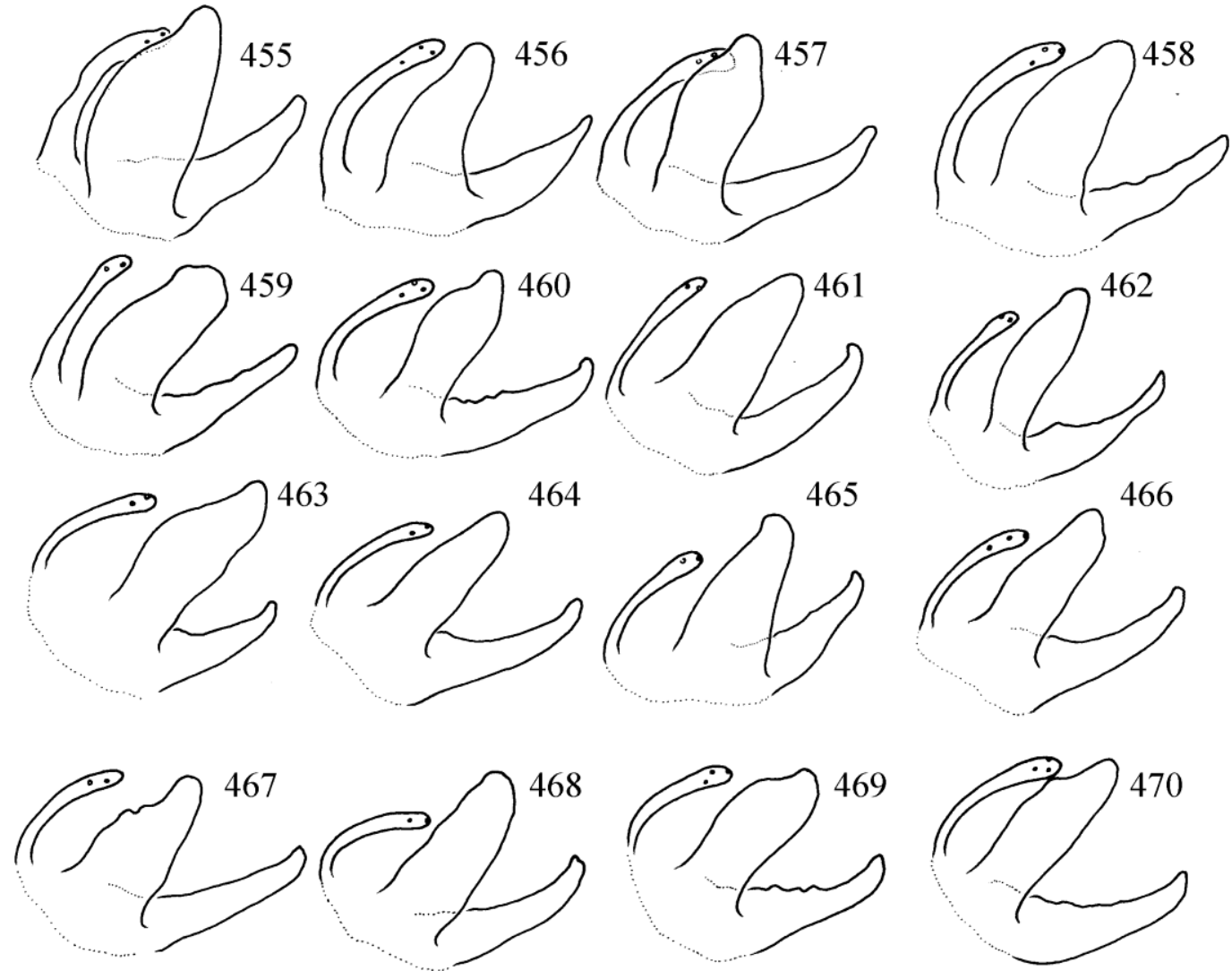

Figures 455-470. Annitella kosciuszki Klapálek, 1907. Superanal genitalic complex in lateral view 


\section{Annitella lateroproducta (Botosaneanu, 1952)}

(Figures 432, 442-448, 471-478, 479-483)

Carpathopsyche lateroproducta Botosaneanu, 1952: 1-15. Annitella lateroproducta (Botosaneanu, 1952): Murgoci \& Botosaneanu 1957: 142, synonymised Carpathopsyche with Annitella.

Annitella chomiacesis lateroproducta (Botosaneanu, 1952): Szczęsny 1979: 260, reduced to subspecies status based on supposed crossing between A. lateroproducta and A. chomiacensis.

Annitella lateroproducta (Botosaneanu, 1952): Botosaneanu 1995: 82: reinstate species status.

Diagnosis. The lateral lobe of the paraproct is longer than the mesal lobe, and the mesal lobe is reduced digitiform as visible both in lateral and ventral view. The sclerotized tip of the aedeagus is blunt. Female anal tube tapering in dorsal view, lateral apodemes of the vaginal sclerite complex rounded laterad directed. The locus typicus of this species is in the Retezat Mts. Romania.

Material examined. Romania, Apuseni Mts. Vadul Crisului, Crisul Rapide, 29. X. 1997, leg. L. Újvárosi (10ิ, OPC). Apuseni Mts. Ic Ponor, spring area of Somesul Cald, 6. XI. 1998, leg. L. Újvárosi $(5 \AA, 49$, OPC). Apuseni Mts. Arieseni, Alboc, 6. X. 1999, leg. L. Theodor (1 $\sigma^{\lambda}, 1$, OPC) Gilau Mts. Jerii Valley, 8. X. 2000, leg. L. Újvárosi (1ठ̄, OPC). Apuseni Mts. Doda Pilii, 3. XII. 2006, leg. L. Újvárosi $\left(60^{\lambda}, 4\right.$,, $\left.\mathrm{OPC}\right)$. Apuseni Mts, Valea lui Dragan, 650m, N46.83119 E22.77093, 20.xi.2008 leg. M. Bálint \&
Tasnádi (1§̂, OPC). Apuseni Mts. Sebes Körös valley, Suncuius, near Izbandis spring, 26. X. 2009 singled leg. J. Oláh \& M. Bálint (7ð̂, OPC). Apuseni Mts, Padis, open stream near pine forested sphagnum bog, $\mathrm{N}^{\circ} 6^{\circ} 35^{\prime} 20.632$ E22 ${ }^{\circ} 45^{\prime}$ 54.857, 5.XI.2011, leg. Gy. Monori, J. Oláh \& L. Szél $(8 \hat{\jmath}, 6$ ㅇ, OPC). Hargitha Mts. Sincraieni, Valea Mare, 6-14. IX. 1993, light trap, (23 ${ }^{2}$, OPC). Ciucaş Mts. $3 \mathrm{~km} \mathrm{~S}$ of Dălghiu, Dălghiu stream, N45³3'00.2”, E2554'43.5”, 970 m, 13.10.2011, leg. Á. Ecsedi, T. Kovács, G. Puskás, $(1 \hat{\jmath}, 1$, , OPC). Caraş-Severin county, Ţarcu Mts., open stream with Salix bushes $6 \mathrm{~km} \mathrm{~S}$ of Poiana Mărului, $1000 \mathrm{~m}$, N4520'47.5”, E22`31'04.6”, 14.10.2011, leg. Á. Ecsedi, T. Kovács, G. Puskás, $(19$, OPC). Caraş-Severin county, Ţarcu Mts., left side brook of open stream on the N slope of Mt. Țarcu, $1500 \mathrm{~m}, \mathrm{~N}^{\circ} 5^{\circ} 17^{\prime} 40.7$ ', E22 31 '44.5”, 14.10.2011, leg. Á. Ecsedi, T. Kovács, G. Puskás, (10̂,1 1 , OPC). CaraşSeverin county, Tarcu Mts., open stream on the N slope of Mt. Țarcu, N45¹7'46.2”, E22³1'41.5”, $1500 \mathrm{~m}$,

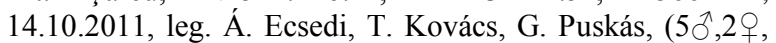
CSNMB). Caraş-Severin county, Semenic Mts., open brook E of Mt. Piatra Goznei, N45 ${ }^{\circ} 10^{\prime} 55.4$ ', E22 ${ }^{\circ} 04$ '01.4”, 1340 m, 15.10.2011, leg. Á. Ecsedi, T. Kovács, G. Puskás, (1 9 , OPC). Gurghiu Mts. near Bucin Pass, Tárnava Mica springs and stream, N: 46³9'16,63” E: $25^{\circ} 16^{\prime} 42,46 ”, 1290$, 30.X. 2014, leg. Z. Baczó, Cs. Balogh, J. Kecskés \& J. Oláh. (1 male; OPC). Hargitha Mts. Filio stream, N: $46^{\circ} 27^{\prime} 03,90^{\prime \prime}$ E: 25³0' 20,10”, 940m, 31.X.2014 leg. Z. Baczó, Cs. Balogh, J. Kecskés \& J. Oláh. (1 male, 1 female; OPC). Dâmbovia county, Bucegi Mts, Hotel Peştera, Ialomiţa, 45²3'54.5”, 25²6'25.1', 1610 m, 07.11.2014, leg. T. Kovács \& G. Magos (1 male, OPC). Sibiu county, Făgăraş Mts, Cârţişoara, Bâlea Stream below the Bâlea Lake, 45³6' 30.4”, 24³7'14.6”, 1940 m, 08.11.2014, leg. T. Kovács \& G. Magos (1 male, OPC).
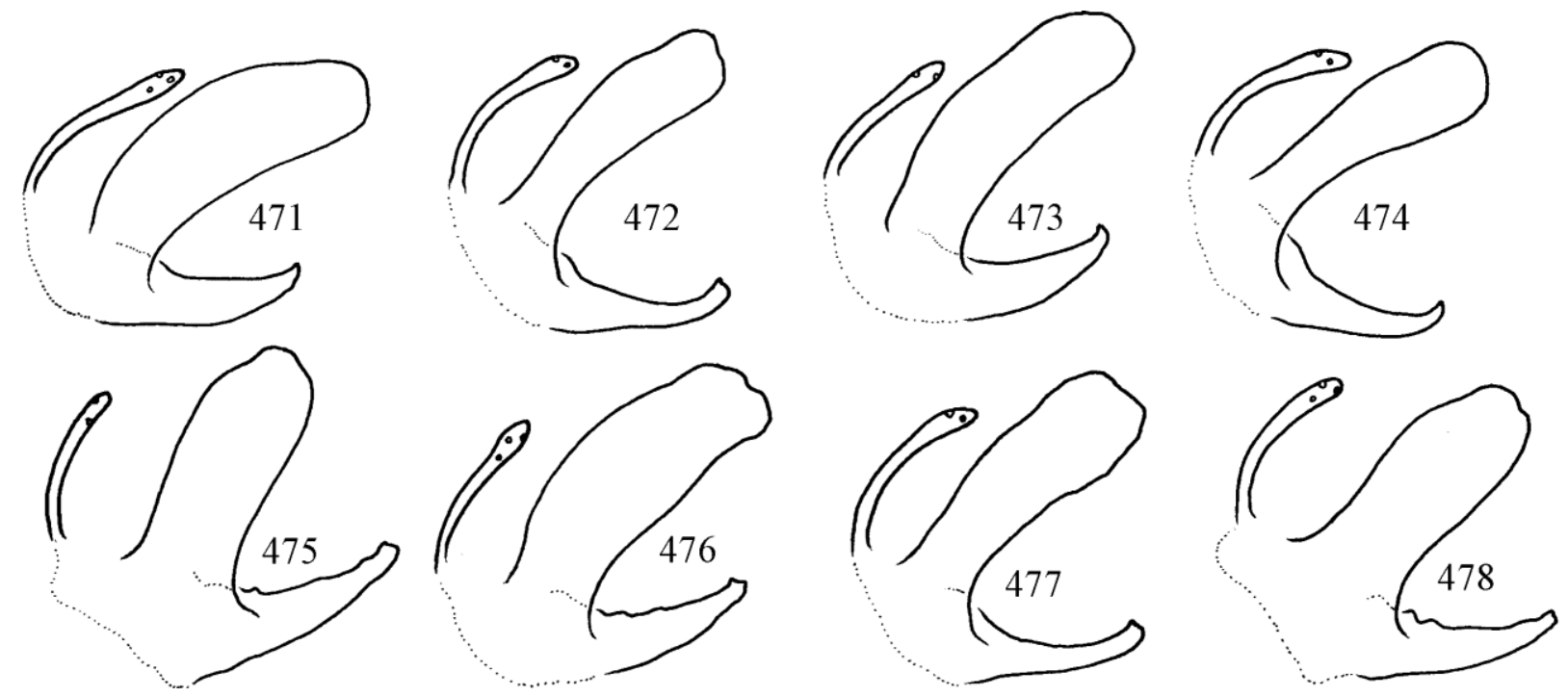

Figures 471-478. Annitella lateroproducta (Botosaneanu, 1952). Superanal genitalic complex in lateral view. 


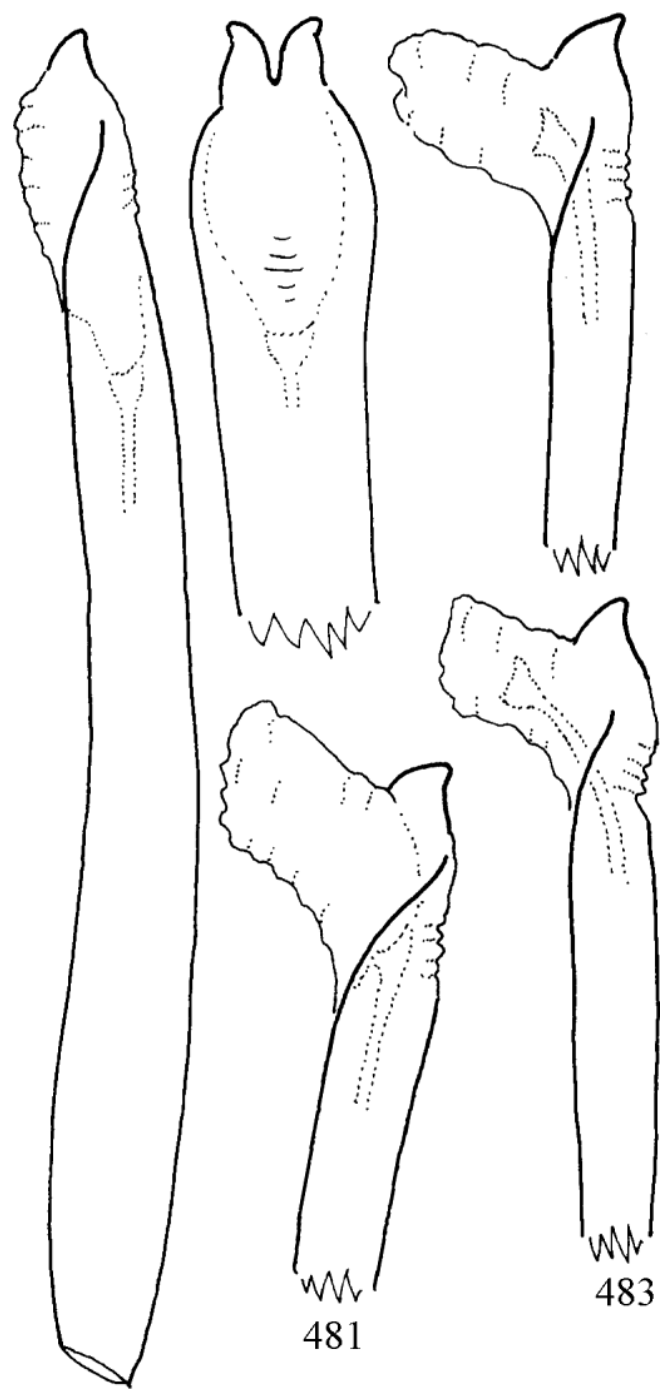

Figures 479-483. Annitella lateroproducta (Botosaneanu, 1952). Aedeagus in lateral and ventral view. $479=$ lateral view, $480=$ ventral view, 481-483 = aedeagus having variously protruded or erected states of endophallus with variously protruded ductus ejaculatorius and gonopore position.

\section{Annitella wolosatka Oláh \& Szczęsny, sp. nov.}

(Figures 435, 440-441, 454, 484-489)

Annitella chomiacensis (Dziedzielewicz, 1908): Szczęsny 1966: 344-346. Misidentification.

Diagnosis. The lateral lobe of the paraproct is very short and directed laterad; the mesal lobe is very robust with rounded lateral margin as visible in lateral view. The sclerotized tip of the aedeagus is less developed, very blunt. Female anal tube with wide mesal excision and produced an addition small mesal lobe on the mesal tip of the lateral lobes in dorsal view; lateral apodemes of the vaginal sclerite complex straight. Reduced male tibial spur formula differs from the other member of the species complex

Material examined. Holotype. Poland, East Carpathians, Bieszczady Mts. at Wołosatka brook, 900m, 28.X.2010, leg B. Szczęsny (1 male, OPC). Allotype. Same as holotype (1 female, OPC). Paratypes. Same as holotype (2 males, OPC). Poland, East Carpathians, Bieszczady Mts. at Wolosatka brook, 850-1000m, 22.X.2014, leg B. Szczęsny (1 male, OPC; 2 males, CSNMB).

Description. Male (in alcohol). Dark brown medium-sized animal with some castanean thoracic sclerites and light body appendages and with yellowish-testaceous wings. Anterior wing with rounded apex and with very long erect spine-like setae present both on the membrane and on the veins; setae on the veins usually stronger. Male tibial spur formula is 013, female tibial formula is 122. Male forewing length is $10 \mathrm{~mm}$, female forewing length is $14 \mathrm{~mm}$.

Male genitalia. Tergite VIII has posterior process long and mesad arching. Segment IX rounded convex in anterior margin in lateral view with very short tergal band. Paraproct bilobed, lateral lobe short and laterad directed mesal lobe very
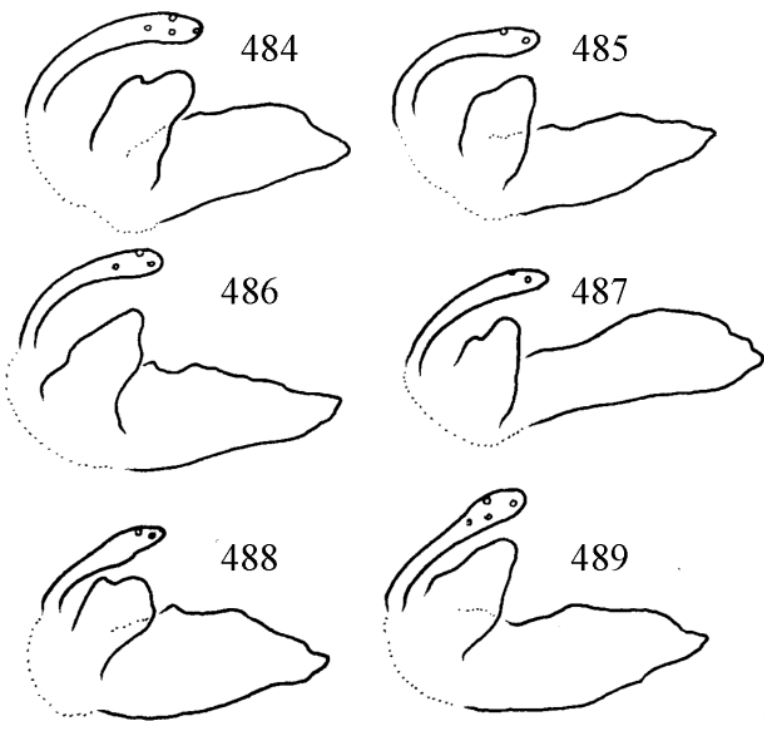

Figures 484-489. Annitella wolosatka, 1907. Superanal genitalic complex in lateral view. 
robust rounded both in lateral and ventral view. Cerci are long digitate. Gonopods fused to segment IX and short. Sclerotized bifid tip of aedeagus very short and blunt.

Female genitalia. Anal tube formed by the fusion of tergite IX and $\mathrm{X}$ is medium long and broad, with very deep mesal excision with addition small mesal lobe on the apical margin in dorsal view. Setose ventroapical lobes of tergite IX rounded. Supragenital plate of segment X glabrous. Median lobe of the vulvar scale (lower vulvar lip) small. Vaginal chamber short. Vaginal sclerite pattern with straight anterior apodemes.

Etymology. This new species was named after the Wolosatka Stream valley in the Bieszczady Mts. where the type material was collected.

Chaetopteryx Stephens, 1829

\section{Chaetopteryx rugulosa species group}

Species delimitation. This species group was established by Malicky et al. (1986) with four known and four new taxa; classified at that time as six species and two subspecies according to the biological species concept. After a quarter of century, applying the phylogenetic species concept and the sexual selection theory we have revised the species group, establishing three subgroups, two species clusters and describing seven new species (Oláh et al. 2012). Malicky (2014) synonymised our three species and questioned all the others, while arguing against the application of the phylogenetic species concept and the sexual selection theory, but without arguments. His nomenclatural acts were rather autocratic contradicting also to his earlier statements (Malicky et al. 1986). Taxa were even not only downgraded to lower taxonomic rank, they were synonymised. $\mathrm{He}$ has overlooked or disregarded our species subgroups and cluster clades both of which were distinguished by older divergences of neutral traits. Malicky's taxonomic actions have been realized without any factual explanations and criticisms regarding the divergence diagnoses of the new clades and species and without giving his own new diagnosis explaining the divergences remained in his new synonymised combined taxa.
Earlier, Malicky (1996) emphasized difficulties in species delimitation among the closely related species in the $C$. rugulosa species group due to high variability of the gross genital structures. He suggested introducing new methods of easeof-use; mtDNA neutral marker to delineate these variable taxa. Examining species of $C$. rugulosa group, routine DNA laboratories released several contradicting mtDNA sequences under the name of the same species, but only by personal communication, never published. The first results of mtCOI DNA survey was published recently (Kucinic et al. 2013). According to Malicky (2014) this sequence analysis confirmed the specific status of several species in the C. rugulosa species group. We understand differently the published Bayesian tree. The mtDNA marker has resolved the three species subgroups we have established by combining both neutral non-adaptive traits of older divergences and non-neutral adaptive traits of contemporary morphological divergences. That is reasonable because some of the neutral, nonadaptive, older divergences of the subgroups could be somehow represented in the loci of the applied marker. At the same time on species level the neutral marker has distinguished only the older species. Only those species were detected, which are otherwise easy to distinguish by traditional gross genital morphology. This blind marker was not sensitive enough to recognise incipient species diverged by adaptive, non-neutral speciation traits. This could be expected because speciation genes, affecting reproductive isolation and expressing the speciation traits of parameres, the paraproct hook formation or of the lateral subapical supporter processes on the aedeagus, cease to flow before the time of gene flow cessation at neutral loci. Their divergence occurred before gene flow between species ceased. This is resulted in discordance between the genealogies of speciation genes and the expressed speciation traits and the neutral loci, giving some insight into the relative role of selection and genetic drift in species formation (Nosil \& Schluter 2011). Certain types of reproductive barriers typically evolve faster (Coyne \& Orr 2004).

Most unexpected is however, that $C$. rugulosa and $C$. noricum were not resolved, but grouped 
together in polytomy. This must be an artefact caused by the applied model thinking and algorithms with multitude of discordant filters. We have clearly documented that these species, having diverged neutral traits, must represent differrent clades and have been diverged long before the contemporary reproductive isolation. Even they belong into different subgroups based on both neutral and non-neutral genital structures, including males and females (Oláh et al. 2012).

During our studies on the members of the $C$. rugulosa species group (Oláh et al. 2012) the phenomenon of the speciation trait was just detected, not defined, not detailed, not recognised properly. That time our knowledge was still rather limited. After having the speciation trait discovered in more limnephilid genera and having more experience, we revisited this species group by repeating the fine structure analysis on both the old and newly sampled populations to meet the initial split criterion. We have prepared diverged structure matrices and here we demonstrate, at the particular species, in more details how remarkably and outstandingly stable are these adaptive diverged traits also at the incipient species of the $C$. rugulosa species group due to reproductive isolation.

Reproductive isolation. Chaetopterygini females are polyandrous, copulating several times with different males. Specimens are often seen in copula in the field frequently surrounded by competing/courting males (Oláh et al. 2012). However, copulation itself does not guarantee sire success, and does not confirm the lack of reproductive barriers. There have been not any systematic field observation carried out how long lasting is the copulation, how frequently it is disrupted by competing male coercion or by female rejection. In captivity and under forced monoandry heterospecific pairs of the following species have started to copulate immediately, as observed under experimental condition (Malicky \& Pauls 2012; Malicky 2014): C. clara, fusca, gessneri, goricensis, marinkovicae, morettii, rugulosa. However the conclusion that their hybrids are more or less fully fertile, develop normally and therefore they are not real species is not well supported. (1) Postzygotic barriers are notoriously difficult to estimate. (2) Relying on fertility alone and ignoring other components of hybrid fitness we surely underestimate the overall postzygotic isolation (Willey et al. 2009). (3) Postzygotic barriers may be expressed over many generations of offspring (Willey 2009). (4) Hybrid incompatibilities may build up later by backcrosses and by recombinations breaking up coevolved gene complexes (Willey et al. 2009). (5) Recent work has demonstrated that in laboratory the experimental design can dramatically affect behavioural isolation measures (Jennings \& Etges 2010, Butlin et al. 2012). (6) Genotype-by-environment interactions and epigenetic processes may influence strongly and variously both pre- and postzygotic isolation. (7) Lack of ecological data to understand how experimental conditions best reflect nature (Butlin et al. 2012). (8) Quantifying hybrid sterility or fertility, a postzygotic measure of reproductive isolation, is very problematic. (9) In laboratory experiments hybrid sterility is quantified very crudely and limited often to the F1 generation. If sterility is manifested in later generations, it is missed to detect (Butlin 2012). (10) In later generations very high sterility was measured: grand- and great grand-offspring from interspecific crosses results in only $2.4 \%$ and $2.7 \%$ of the number of descendent typical of conspecific pairing (Wiley et al. 2009). (10) Hybrid breakdown when first generation hybrids are viable and sterile, but offspring in next generation is not. (11) Focus on F1 reproductive success may prevent proper assessment of the underestimation of postzygotic isolation.

The strong polyandry, strong sexuality, and extended copulation observed in nature are the primary signs of intense sexual selection in the chatopterygini tribe. Mating and associated preand post-mating processes are energetically demanding, time-consuming and the vulnerability to predators increasing during long copulation. But females may gain both material and genetic benefits from multiple mating. Male accessory gland substances could be transferred increasing female fitness. Our knowledge is very limited about the various possible mechanisms already detected and demonstrated during sexual selection processes in closest relatives, among the Lepidoptera. From the mere fact that we find many pairs in copula in a field population, even in sympatry of secondary 
contact, it does not follow that reproductive isolation is weak. Reproductive isolation may be present and works also in copula. The various forms of premating barriers of reproductive isolation including species-assortative female mate preferences may be present and active. Several possible mating and postmating, prezygotic barriers, like cryptic female choice, sperm competition, and gametic isolation may also work. In secondary contact, females in heterospecific pairs may have extra-pair copulations with conspecific males (Sætre \& Sæther 2010). In case of significant gene flow both extrinsic and intrinsic postzygotic barrier may function. Male hybrids may be less successful in obtaining mate, especially if polyandry is so strong and many males are around, what we observe in the field, not speaking about the lack of any data on hybrid fitness.

Phylogenetic species. Based on statement that all the listed Chaetopteryx species produces more or less fully fertile hybrid, Malicky (2014) deduced the lack of reproductive barriers between these species in the sense of cladistics and concluded that they are not real species, according to the strict cladistic definition. He is not aware of the fact that so many cooperative mechanisms work together in order to maintain reproductive barriers semipermeable to gene flow while species can differentiate despite ongoing interbreeding (Hausdorf 2011). Confused further, Malicky claims that divergence, that is bifurcation in cladistics, "the origin of two new species while the former species is supposed to disappear", is a brilliant methodical trick without reality and anything which may be hybridized is not a separated species at all. From these statements it is not clear which kind of species concept was applied during his taxonomical actions on the Chaetopteryx species. Species-concept debates, "vainly beating the air", need not impede progress in science (Winkler et al. 2007). We believe that the opposite is suggested by the phylogenetic species concept of cladistics origin. Clades are not lineages, like bifurcating is not budding and a species is not an entire lineage, but only a segment of a lineage, "a branch in the line of descent". What else could be the species if not lineages, if we accept both the time and the space as real dimensions? A lineage is a population extended trough time or a population is a lineage at a moment in time. Incipient species starts lineages with an ancestral-descendant sequence of populations (deQueiroz, 2011).

Darwin's concept equates species with "branches in the lines of descent". This was confirmed by the evolutionary synthesis and detailed by Hennig's phylogenetic systematics and enriched by Mayr's interbreeding populations, reproductively isolated from other such groups. In practice, the old taxonomic component that species category is a taxonomic rank was however retained with statement about the artificial nature of this species category. Some authors misinterpreted Darwin's statement suggesting that species are not real. But species are real segments of lineages. They do exist in nature and not only in the human mind (deQueiroz 2011). The old taxonomic rank was dissolved into or among the various aspects of Post-Darwinian species ranking criteria: phenetic cluster, reproductive barrier, mate recognition or fertilization system, distinct niche or adaptive zone, fixed character state differences, monophyly, exclusive coalescence of alleles (deQueiroz 2011). It is promising that with these developments the artificially separated process-based and pattern-based species concepts (Winkler et al. 2007) has started to unify old misunderstandings. Today post-neo-darwinism is going to transfer species from the hierarchy of taxonomic ranks to the hierarchy of biological organisation and clearly confirm that phylogenetic species are the smallest diagnosable cluster of individual organisms within which there is a parental pattern of ancestry and descent.

Speciation traits. Parameres are not contemporary speciation trait in Chaetopteryx rugulosa species group, not diverging consistently on species level. They exhibit older divergences at subgroup level: (1) $C$. rugulosa subgroup has probably the ancestral state of parameres characterized by the bunch formation; (2) C. schmidi subgroup has evolved a single primary stout spine, possibly representing the enforced terminal of the paramere shaft which is accompanied with 
2-3-4 less developed spine-like setae; (3) $C$. irenae subgroup has evolved elongated spine-like seate in various species specific quantity. Subgroup level evolution of paramere is confirmed by parallel subgroup level evolution of periphallic and phallic male as well as external female genitalic structures of older divergences: (1) low cerci, partial lack (possibly incomplete lineage sorting) of supporting sclerite ridges on aedeagus and presence of setose lateral lobes on female tergite IX in C. rugulosa subgroup; (2) high cerci, partial lack of supporting sclerite ridges on aedeagus, lack of setose lateral lobes on female tergite IX in C. schmidi subgroup; (3) very high cerci, presence of supplementary digital process on superanal complex, presence of supporting sclerite ridges on aedeagus, very developed setose ventrolateral lobes on female tergite IX in $C$. irenae subgroup.

In the $C$. rugulosa species group the lateral processes located subapicad on the aedeagus are the main speciation traits together with the apical hook formation of parproct. The curvature of the paraproct hook has an important function in the copulatory process. The heavily sclerotized hooks with serrated dorsomesal edges deeply penetrate into the female anal tube anchoring against the internal sclerite inside the anal tube (Oláh et al. 2012). The anal tube and the paraproct hook formation may coevolve. Both the length and the shape of the anal tube as well as the hook curvature diverged and are rather stable having diagnostic value at least in the $C$. schmidi subgroup.

The contemporary reproductive isolation is realised mostly in the species specific development of the lateral subapical supporting processes on the aedeagus. Their shape divergence is very pronounced from a small bud of $C$. schmidi in the C. schmidi subgroup and C. zalaensis in the C. rugulosa subgroup, $C$. rugulosa cluster to the variously shaped large vertical plates in the $C$. irenae subgroup. Together with the membranous eversible endophallus they function as a complex trait of reproductive isolation. They are serving as the speciation trait in these incipient species. The flexible and eversible endophallus is the actual terminal head of aedeagus, the intromittent organ, fitting and filling the vaginal chamber with species specific configuration. When fully erected inside the negative template of vaginal chamber it may act as a key-and-lock mechanism of clasping device with interlocking function holding male and female together during the long copulation. This interlocking mechanism may also serve to keep the gonopore at the end of the ejeculatory duct firmly attached to the sclerotized spermathecal process of the dorsal vaginal sclerite complex. This firm coupling is critical for successful insemination. The endophallus itself is composed of species specific lobe structure combined of lateral, dorsal and ventral lobe systems, but visible highly varying when we observe them due to the various copulatory/erection states of the examined specimens of the same species. The shape of the eversible endophallus is highly erection dependent. These soft structures have species specific shapes but difficult to apply in routine taxonomic studies because it requires laborious procedures to swell and stabilize their shape.

In contrary, the lateral processes are more stable! These lateral processes perform sophisticated species specific supporting action, giving additional holding power to the eversible and erected endophallus during the long copulation. They may function also as a stimulating titillator to purchase male's internal courtship in order to influence females. Their outer membrane is more rigid compared to the endophallic membranes. Rigidity is however variable, especially the apical portion variously inflatable. Their central function in the sexual selection processes either through internal courtship and/or through cryptic female choice is directly demonstrated by the diversity evolution of various sclerotizations evolved to strengthen their holding and stimulating function. In most species only the basal articulation ring is sclerotized; in C. kamnikensis and C. psunjensis sp. nov. the sclerotization is developed into a basal articulation tube. In the $C$. noricum species cluster and in the $C$. irenae species subgroup an independent ventral pair of supporting sclerites evolved with various ridge formations. In $C$. karima sp. nov. one pair of sclerotized supporting 
sclerites developed on the dorsum and one pair in the ventrum. In C. psunjensis sp. nov. a short dorsal ridge of supporting sclerite evolved. These lateral processes on the aedeagus head, preceding the eversible endophallus, are not varying, at least as regards the shape of the basal sclerotized or more rigid region of the process; These regions are stable with species specific shape and size development and less dependent on the erection state.

Sympatry. The range of variation is well documented on diverged trait matrices of the particular species: (1) in pure population the individual shape variation is very small and remains in the range of species specific trait configuration; (2) we have found some variation in a few populations of secondary contact zone, where two incipient species live together. We agree with Malicky (2014) that isolation is much underestimated as an evolutionary factor. Theoretically, it is also possible that species evolves in sympatry and allopatry is secondary. Studies detected significant signals of gene flow during species formation. Divergence develops at some speciation genes, even if there is gene exchange for other genes (Hey 2006). Divergence with gene flow can be a real model and a common process of speciation in nature. Biogeography does not always produce the same pattern than population genetics. It is more productive to understand processes by modelling and measuring quantities, such as gene flow and selection, than just by categorizing like sympatric or allopatric speciation. Sympatric speciation is an infinitesimal end point of a continuum (Fitzpatrick et al. 2008).

Everybody agree with Malicky not to supply a specific name for every specimen which looks slightly different. But taxonomy is to find stable, consistent trait. Half century ago new systematics, merging taxonomy with genetics, promised to find speciation genes/traits to delimit incipient species on large scale of the taxonomic level. Unfortunately this is not realized in practice; gene mapping remained mostly a theoretical adventure limited to some model organisms. Instead of real progress the neutral markers and barcoding seduced geneticists, but produced less. The deprived taxonomy without funding was unable to search sophisticated phenomic traits with fine structure analysis on larger scale.

There is not any examination carried out on $C$. rugulosa group to search prezygotic barriers to prevent interspecific gametes from fusing during assortative mating or in the sexual selection mechanisms. Simply we are faced with the final product of the selection. The discovered speciation trait may represent a possible premating and mating barrier in reproductive isolation. No field study was carried out either on postmating or postzygotic barriers of the hybridization to reduce fitness of hybrids. We have no data on demography, migration census, effective gene flow, hybrid viability, hybrid fertility, extrinsic or intrinsic postzygotic incompatibilities, character displacement, and species reinforcement. It is generally known that species recognition is cooperated and concerted by various mechanisms of premating, mating, and postmating barriers. Individuals can recognise each other as mating partners on a wide temporal scale: before mating, during copula and after mating. Coerced copulation itself could be frequent without real sire success. In reality all of these mechanisms are studied and knowledge accumulated mostly on model organisms. Fortunately, taxonomy can delimit newly born taxa with phenomics without detailed knowledge of genetics in case if we have speciation traits discovered.

Incipient species of C. rugulosa species group. Based upon the relevant principles discussed above in this species group, on species delimitation, reproductive isolation, phylogenetic species, speciation traits and sympatry/allopatry relations, we may conclude that the incipient species delineation is possible without detailed molecular knowledge of the processes of reproductive isolation. This young species group under contemporary speciation process exhibits an intense rate of peripatric speciation with very complex pattern on the peripheries of the ancestral species of $C$. prealpensis and $C$. balcanica sp. nov.

The ancestral species of $C$. prealpensis with three peripatric species (C. kamnikensis, $C$. rugulosa, C. zalaensis) has speciation trait of the 
lateral process on the aedeagus very stable. Stability was recorded on the entire distributional area covering countries of Croatia, Slovenia, Austria, and Hungary, based upon the examination of 57 populations. Similarly we have found the speciation trait of both the head structure of the aedeagus as well as the shaft and setal pattern of the paramere remarkably stable in 106 populations of the Potamophylax nigricornis, the ancestral species of the P. nigricornis species group with seventeen peripatric species (Oláh et al. 2013c).

The putative ancestral species of $C$. balcanica sp. nov. has produced seven peripatric incipient species (C. schmidi, C. tompa sp. nov., C. kozarensis sp. nov., C. karima sp. nov., C. psunjensis sp. nov., C. papukensis, C. mecsekensis). Some of these species requires further study on more specimens from more populations in order to confirm their phylogenetic relations. Especially those species need more studies which have evolved in sympatry under significant gene flow or exposed to reinforcement in hybrid contact zones. The status of $C$. balcanica sp. nov. needs comprehensive studies with more specimens from more populations in Serbia and Bosnia-Herzegovina. Detailed fine structure study is promising on supporting sclerites of the aedeagus and on the paraproctal apical hook formation as well as on the female anal tube and vaginal sclerite complex. Probably more incipient species wait to be discovered and described on low or medium elevations of the isolated southern mountain ranges along the distributional area in Serbia and BosniaHerzegovina.

\section{Chaetopteryx schmidi species subgroup}

Chaetopteryx schmidi species subgroup. Oláh et al., 2012: 59.

Chaetopteryx schmidi subgroup is characterized by neutral traits of high cerci and the lack of setose lateral lobes on female tergite IX. In older divergence the parameres have evolved a pattern of modified terminal setae reduced to a single primary stout spine-like seta, enforced strongly, and accompanied with less developed spine-like setae; this stout spine-like primary seta appears as the continuation of the paramere shaft; contemporary species specific divergence evolved in the curvature and length of this primary seta; the aedeagus is without supporting sclerite ridges on the ventrum, except present probably as incomplete lineage sorting at $C$. karima sp. nov. and $C$. psunjensis sp. nov.

\section{Chaetopteryx balcanica Oláh, sp. nov.}

(Figures 490-507, 508-523, 524-533)

Chaetopteryx schmidi Botosaneanu, 1957: Oláh et al. 2012: 62. All the specimens from Serbia and Bosnia-Herzegovina misidentified!

Diagnosis. This new species having stout spine-like terminal shaft on the paramere as well as having no setose lateral lobes on female tergite IX belongs to the $C$. schmidi subgroup, but differs from all the know species by having combined character state: (1) Sallow curvature on paraproctal hook, not deep curving more anterad as at C. schmidi; (2) Variously sized lateral process, but without any sclerotized basal tube; only a sclerotized basal ring is present permitting a longitudinal position of the inflated and protruded membranous process; in many specimens the membranous process is withdrawn inside the cylinder, not visible at all; at $C$. schmidi the sclerotized basal tube is present and producing and supporting a perpendicular position of the protrudable and inflatable membranous posterior part of the lateral process; at $C$. papukensis the sclerotized basal tube is present, but almost longitudinal and the membranous process is not retractable entirely; there are no any specimens without lateral process. (3) Primary spine long and curved like at $C$. papukensis, but paramere shaft is not triangular in dorsad view. (4) Female anal tube quadrangular with less protruded inner sclerite. We presume that $C$. balcanica sp. nov. is the ancestral species of the $C$. schmidi subgroup widely distributed from East Serbia through Bosnia-Herzegovina, but we need to examine more specimens from more populations to confirm its relations.

Material examined. Holotype. Serbia, Derdap Mts. Donji Milanovac, Grgeci spring and its outlet in a beech forest, 500 m, N44 $28^{\prime}$ E22 ${ }^{\circ}$ 02', 13.X.2006, leg. L. Dányi, J. Kontschán \& D. Murányi (1 male, in copula with the allotype, 

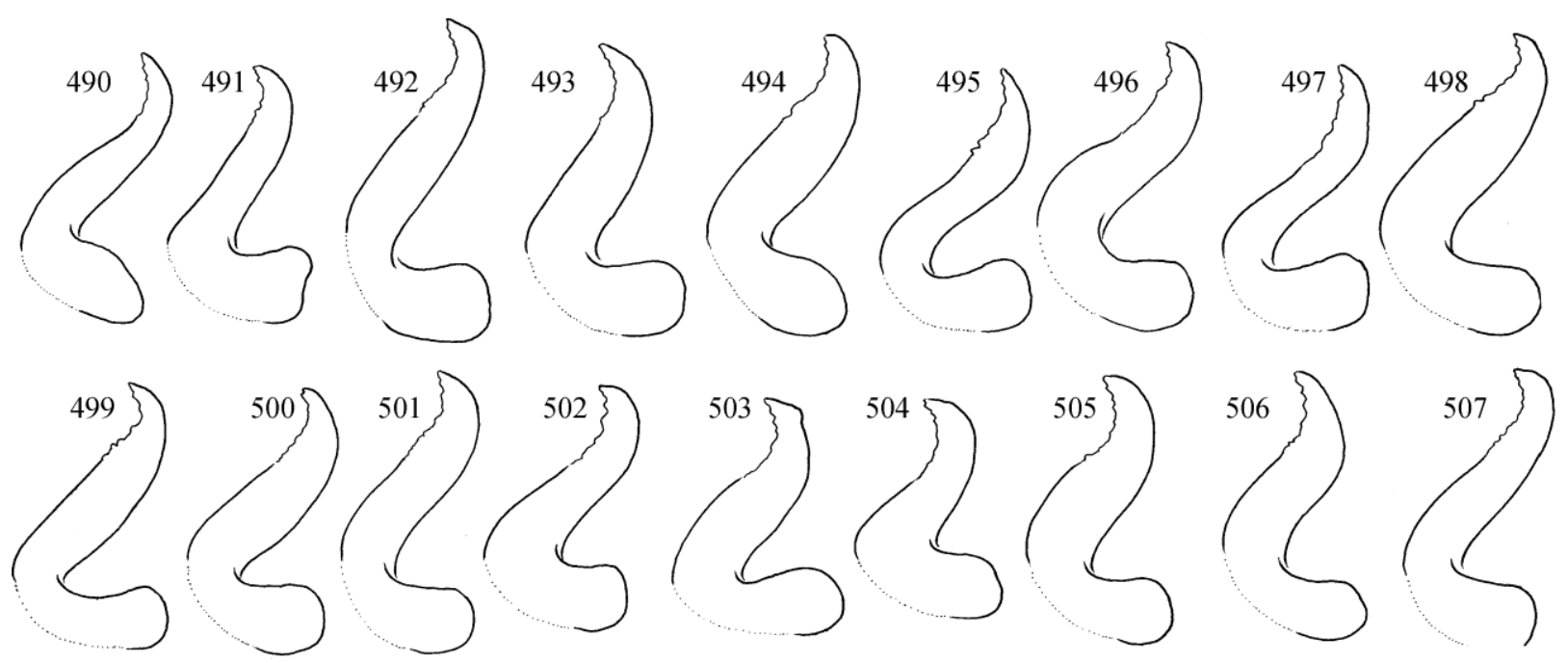

Figures 490-507. Chaetopteryx balcanica Oláh, sp. nov. $490=$ dorsal branch of the holotype paraproct in lateral view, $491-507=$ dorsal branch of paratype paraprocts in lateral view.
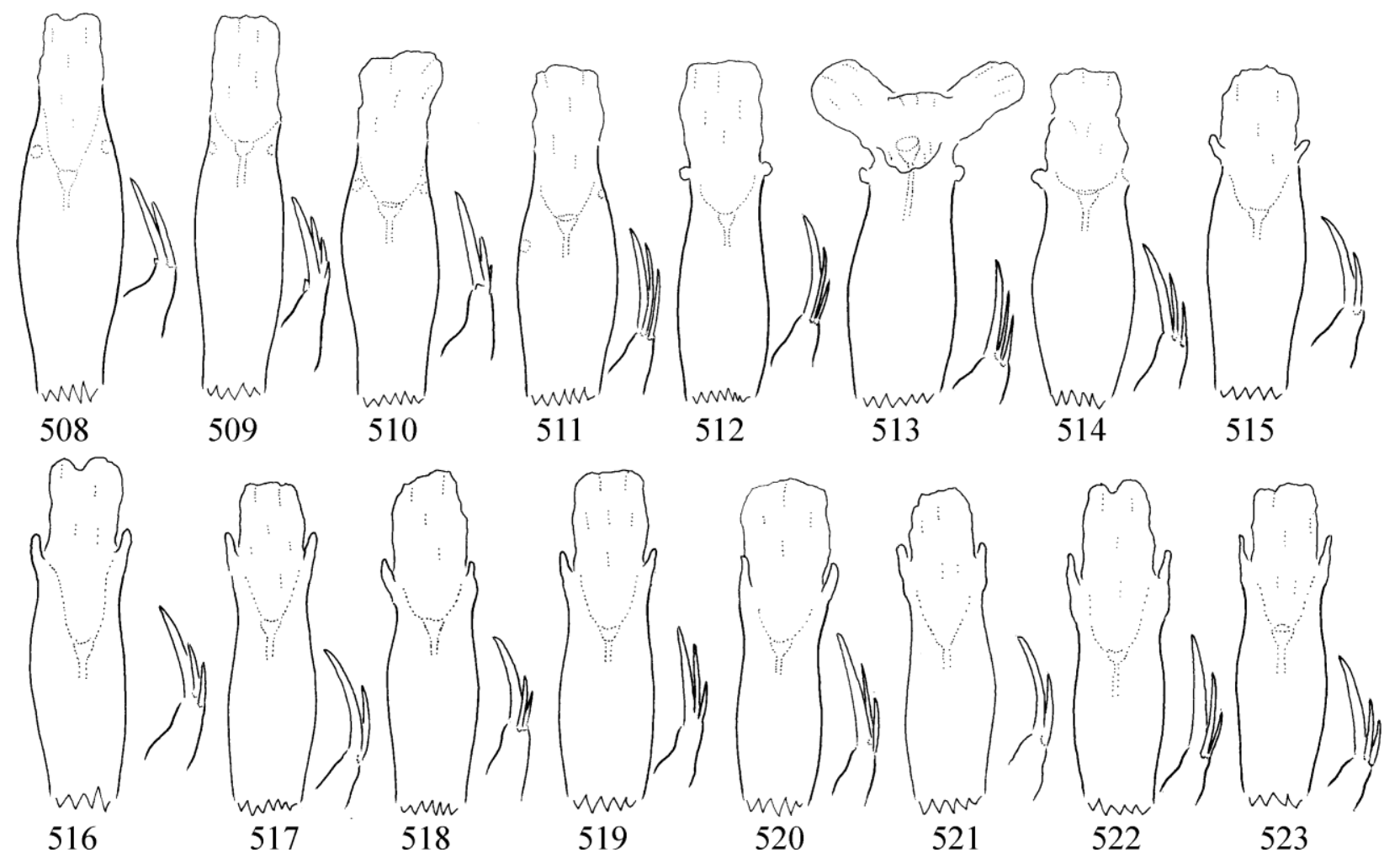

Figures 508-23. Chaetopteryx balcanica Oláh sp. nov. $508=$ aedeagus and paramere of the holotype in dorsal view, 509-523 = aedeagus and paramere of paratypes in dorsal view. 


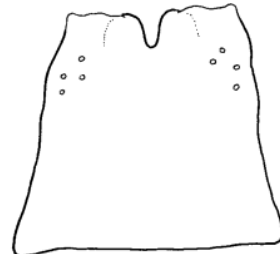

524

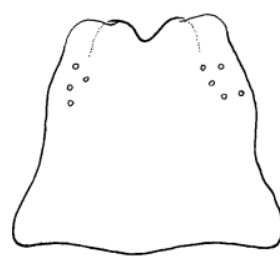

529

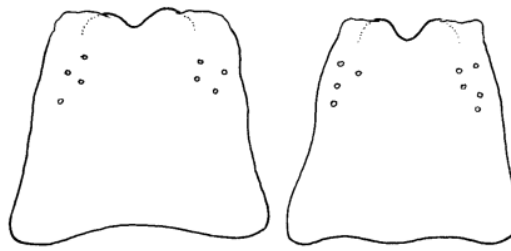

525
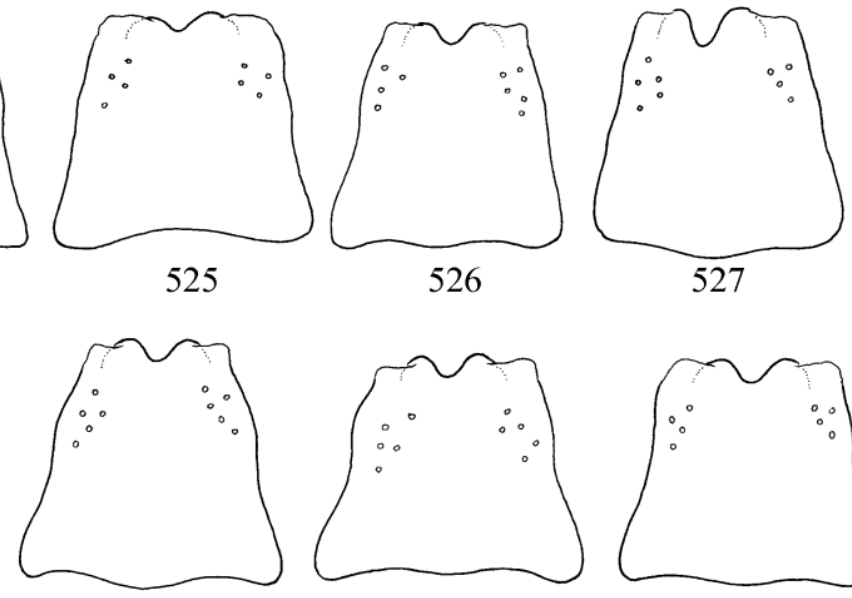

530
526

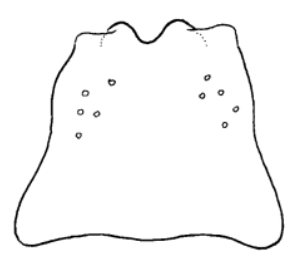

531

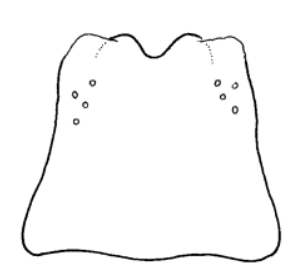

532
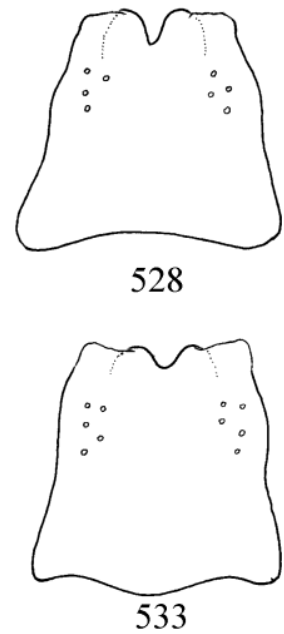

Figures 524-533. Chaetopteryx balcanica Oláh sp. nov. $524=$ female anal tube of the allotype in dorsal view, $525-533=$ female anal tube of paratypes in dorsal view.

HNHM). Allotype. Same as holotype (1 female in copula with the holotype; HNHM). Paratypes. Same as holotype (1 male, 1 female, OPC). Miroc, D. Milanovac, Stream Supljanka, 8.X.1984, leg. Branceli (1 female, PMS). Pesaca, Donji Milanovac, 9.X.1986, leg. I. Sivec, \& B. Horvát (1 male, PMS). Popadija, Donji Milanovac, 9.X.1986, leg. I. Sivec, \& B. Horvát (1 male, PMS). Derdap Mts. Golubinje, stream valley with young forest, $\mathrm{N}$ of the village, $88 \mathrm{~m}, \mathrm{~N} 44^{\circ}$ 30’59.6' E22 ${ }^{\circ}$ 12’41.5”, 13.X.2006, leg. L. Dányi, J. Kontschán \& D. Murányi (6 males, HNHM). Derdap Mts. Dobra, Reka Pesaca, beech forest with stream, $386 \mathrm{~m}, \mathrm{~N} 44^{\circ}$ 34,670 E21 ${ }^{\circ}$ 59,250, 28.X.2010, leg. L. Dányi, J. Kontschán \& Zs. Ujvári. Murányi (2 males, 1 female; HNHM). BosniaHerzegovina, Kravica, Zvornik, 5.X.1986, leg. I. Sivec \& B. Horvát (1ڤ̂, PMS). Dobrovci,Gracanica, 430m, 11.X.1990, leg. B. Horvát \& I. Sivec, (1 male, 3 female; PMS; 1 male, 1 female; CSNMB). Blagijevici, Ozren Planina, $390 \mathrm{~m}$, 12.X.1990, leg. B. Horvát \& I. Sivec, (1 female, PMS). Kamensko, River Krivaja, 15.X.1990, leg. B. Horvát \& I. Sivec, (2 males, PMS). Cunista, River Krivaja, 450m, 15.X.1990, leg. B. Horvát \& I. Sivec, (1 male, 1 female; PMS). Skender, vakuf, 820m, 19.X.1990, leg. B. Horvát \& I. Sivec, (2 males, PMS).

Description. Male (in alcohol). Light brown medium-sized animal with light body appendages and with yellowish-testaceous wings. Anterior wing with rounded apex and with very long erect spine-like setae present both on the membrane and on the veins; setae on the veins usually stronger. Tibial spur formula is 033. Forewing length is $8 \mathrm{~mm}$.

Male genitalia. Posterodorsal spinate area of vestitural noncellular microtrcihiae on segment VIII and its mesal light band well developed. Segment IX with short, bridle-like dorsum and longer ventrum; anterior margin rounded convex with long antecosta; posterior margin concave, midlateral sclerotized angle of tergite IX pronounced. The pouch-like concavity of segment X long. Cerci high. Apical hook of the paraproctal complex with pointed apex, with shallow curvature. Membranous subanal lobe long. Gonopods short with apical flap developed and turning mesad resulting in a single short narrowing lobe in lateral view. Phallic organ composed of short rim-like phallic apodeme, short tube of phallotheca, short endotheca, well-developed aedeagus and medium long elongated parameres; paramere with stout curving primary spine (enforced terminal shaft formation), 2 secondary spines of modified setae; simple sclerotized cylinder of the aedeagus bellied in dorsal view; supplied with a pair of short digitate lateral processes probably retractable, only the very basal ring of the lateral process is sclerotized and membranous after; ejaculatory duct ending with sclerotized gonopore opening into a large trilobed endophallic membranous structure. The sclerotized basal tube of aedeagus is simple cylinder without any enforced supporting sclerotized ridges or flanges.

Female genitalia. Anal tube formed by the fusion of tergite IX and $\mathrm{X}$ is medium long and broad, quadrangular in dorsal view; apical margin with various $V$-shaped excision. The internal sclerite of the anal tube, the vestigial tergite $\mathrm{X}$ is not protruded posterad. Setose ventroapical lobes of 
tergite IX lacking. Supragenital plate of segment $\mathrm{X}$ subtriangular in ventral view. Median lobe of the vulvar scale (lower vulvar lip) half long as the lateral lobes. Vaginal chamber medium sized reaching to the middle of sternite VIII. Vaginal sclerite pattern with rounded mesad turning anterior apodemes.

Etymology. This new species was named after its wide distribution in the Balkan Peninsula.

\section{Chaetopteryx karima Oláh, sp. nov.}

(Figures 534-536)

Chaetopteryx papukensis Oláh \& Szivák, 2012 in Oláh et al. 2012: 62 (partim). Misidentification!

Diagnosis. This single male was collected in a small forest stream where an unknown Leuctra species was also collected in previous spring, still waiting to describe. Earlier, without experiences on speciation strait and the application of fine structure analysis we have determined this specimen and listed as a paratype of $C$. papukensis (Oláh et al. 2012). Although the female is unknown, the strongly developed paramere spine clearly relates this new species into the $C$. schmidi subgroup. The lack of setose lateral lobes on female tergite IX would further confirm the phylogeny of this interesting species. The presence of well elaborated supporting sclerite system of flange and ridge formation on the aedeagus is present only in $C$. irenae subgroup and in $C$. noricum species cluster. The detection of this much specialised trait of deeper/older divergence in C. schmidi subgroup seems discordant and explainable by incomplete lineage sorting. Having this specific supporting sclerite system on the aedeagus present this is a well diverged new species, however female traits of lateral setose lobe and anal tube formation would give more information about its relations.

Material examined. Holotype. Bosnia-Herzegovina, Banja Luka region, Kozara Mts, forest brook below the Vrbaška - Kozarac road, 4502.480', 16 ${ }^{\circ} 54.266^{\prime}, 560 \mathrm{~m}$, 07.11.2012, leg. T. Kovács \& G. Magos (1 $\overbrace{}^{\lambda}$, OPC).

Description. Male (in alcohol). Light brown medium-sized animal with light body appendages and with yellowish-testaceous wings. Anterior wing with rounded apex and with very long erect spine-like setae present both on the membrane and on the veins; setae on the veins usually stronger. Tibial spur formula is 033. Forewing length is $12 \mathrm{~mm}$.

Male genitalia. Posterodorsal spinate area of vestitural noncellular microtrichiae on segment VIII and its mesal light band well developed. Segment IX with short, bridle-like dorsum and longer ventrum; anterior margin rounded convex with long antecosta; posterior margin concave, midlateral sclerotized angle of tergite IX pronounced. The pouch-like concavity of segment X long. Cerci high. Apical hook of the paraproctal complex with blunt apex. Membranous subanal lobe long. Gonopods short with apical flap developed and turning mesad resulting in a single short pointed apex in lateral view. Phallic organ composed of short rim-like phallic apodeme, short tube of phallotheca, short endotheca, well-developed aedeagus and medium long parameres; paramere with stout curving primary spine (enforced terminal shaft formation), 2 secondary spines and 1 tertiary spines of modified setae; aedeagus supplied with a pair of digitate lateral processes directed oblique upward, the very basal part of the lateral process sclerotized and membranous after; ejaculatory duct ending with sclerotized gonopore opening into a large trilobed endophallic membranous structure. The sclerotized basal tube of aedeagus is enforced by elaborated system of supporting sclerite flange and ridge system composed of a shorter pair of dorsal flanges directly connecting to and enforcing the sclerotized basal ring of the lateral process and a longer pair of ventral flange connecting to basal position of the membranous endophallus.

Etymology. karima from "karima" flange in Hungarian, refers to the double pairs of flange ridge formation on the aedeagus.

\section{Chaetopteryx kozarensis Oláh, sp. ov.}

(Figures 537-539, 540-542)

Chaetopteryx papukensis Oláh \& Szivák, 2012 in Oláh et al. 2012: 62 (partim). Misidentification! 


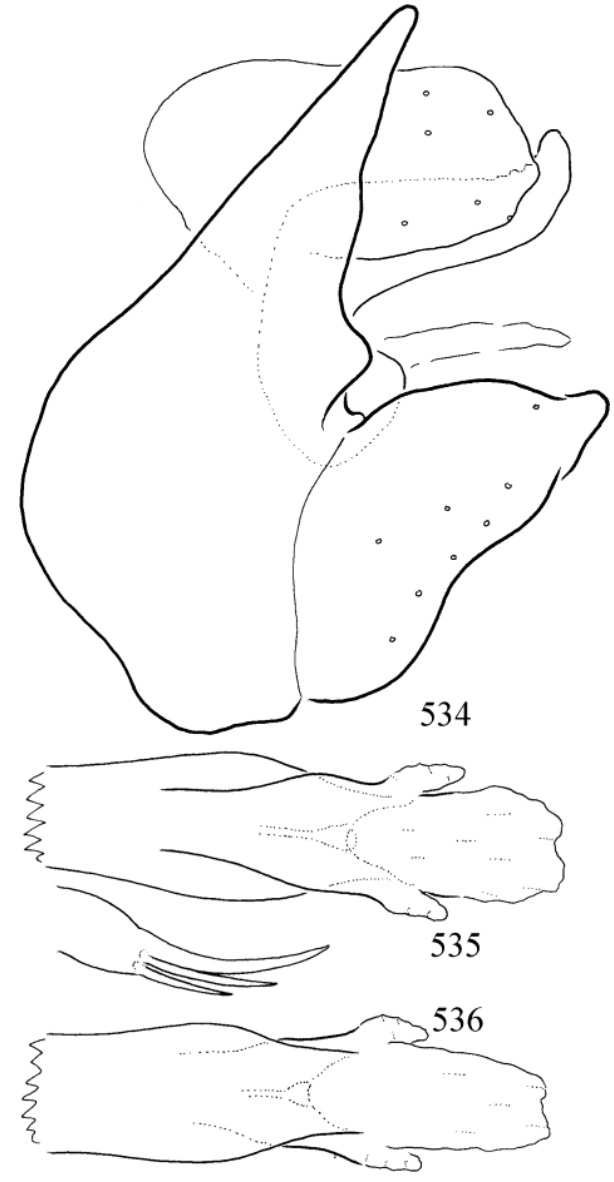

Figures 534-536. Chaetopteryx karima Oláh sp. nov. $534=$ male genitalia in lateral view, $535=$ aedeagus and paramere in dorsal view, $536=$ aedeagus in ventral view.

Diagnosis. Most close to C. papukensis, but differs by having apical hook formation of the dorsal branch of the paraproct blunt, not pointed and the curvature is very shallow. The most striking divergence from all the known female anal tube in the species group is detected in the dorsal configuration. This is why the female was designnated as holotype. The unique dorsal profile of the anal tube is characterized by a deep V-shaped excision; however we do not know how the anchoring pressure may modify the shape of the excision by moving the internal sclerite backward. It is actually not known whether this internal sclerite is movable at all or not. The bilobed posterior end of the internal sclerite is in protruded state in all of the examined hundreds of $C$. papukensis. This may suggest that the internal sclerite, that is the vestigial tergite $\mathrm{X}$ fixed withdrawn inside the anal tube is not movable even under the long anchoring pressure of the paraproctal hook.

Material examined. Holotype. Bosnia-Herzegovina, Banja Luka region, Kozara Mts, forest edge spring $1 \mathrm{~km} \mathrm{~S}$ of peak Lisina, 4457.773', 1658.342', 680 m, 7.XI.2012, leg. T. Kovács \& G. Magos (1 female, OPC). Allotype. Same as holotype (1 male, OPC). Paratypes. Same as holotype (3 males, 4 females; OPC, 1 male, 1 females; CSNMB).

Description. Male (in alcohol). Light brown medium-sized animal with light body appendages and with yellowish-testaceous wings. Anterior wing with rounded apex and with very long erect spine-like setae present both on the membrane and on the veins; setae on the veins usually stronger. Tibial spur formula is 033. Forewing length is $10 \mathrm{~mm}$.

Male genitalia. Posterodorsal spinate area of vestitural noncellular microtrichiae on segment VIII and its mesal light band well developed. Segment IX with short, bridle-like dorsum and longer ventrum; anterior margin rounded convex with long antecosta; posterior margin concave, midlateral sclerotized angle of tergite IX pronounced. The pouch-like concavity of segment X long. Cerci high. Apical hook of the paraproctal complex with blunt apex. Membranous subanal lobe long. Gonopods short with apical flap developed and turning mesad resulting in a single short narrowing lobe in lateral view. Phallic organ composed of short rim-like phallic apodeme, short tube of phallotheca, short endotheca, well-developed aedeagus and medium long elongated parameres; paramere with stout curving primary spine (enforced terminal shaft formation), 2 secondary spines and 1 tertiary spines of modified setae; aedeagus supplied with a pair of digitate lateral processes directed oblique upward, the very basal ring of the lateral process sclerotized and membranous after; ejaculatory duct ending with sclerotized gonopore opening into a large trilobed endophallic membranous structure. The sclerotized basal tube of aedeagus is simple cylinder without any enforced supporting ridges or flanges.

Female genitalia. Anal tube formed by the fusion of tergite IX and $\mathrm{X}$ is medium long and broad, slightly narrowing posterad; apical margin 


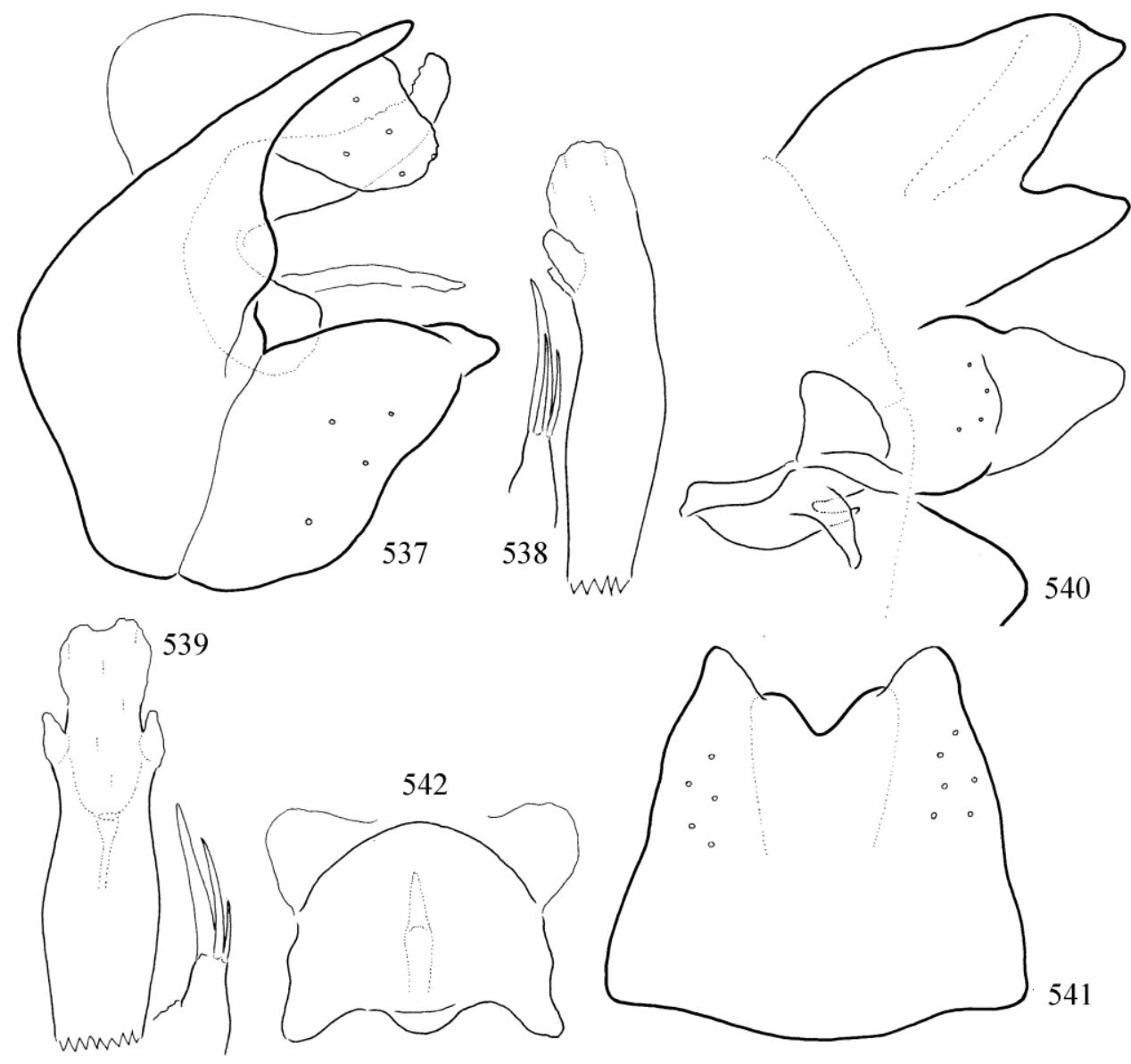

Figures 537-542. Chaetopteryx kozarensis Oláh, sp. nov. $537=$ male genitalia in lateral view, $538=$ aedeagus and paramere in lateral view, $539=$ aedeagus and paramere in dorsal view. $540=$ female genitalia with dorsal vaginal sclerite complex in left lateral view, 541 = female anal tube in dorsal view, $542=$ dorsal vaginal sclerite complex in dorsal view.

with deep V-shaped excision. Setose ventroapical lobes of tergite IX lacking. Supragenital plate of segment $X$ subtriangular in ventral view. Median lobe of the vulvar scale (lower vulvar lip) half long as the lateral lobes. Vaginal chamber medium sized reaching to the middle of sternite VIII. Vaginal sclerite pattern with rounded slightly laterad turning anterior apodemes.

Etymology. This new species was named after the mountain range where the type material was collected.

\section{Chaetopteryx papukensis Oláh \& Szivák, 2012 stat. restit.}

(Figures 543-550, 551-557, 558-570)

Chaetopteryx rugulosa mecsekensis Nógrádi, 1986: Oláh 2010: 98. Misidentification.
Chaetopteryx papukensis Oláh \& Szivák, 2012: 60-62. Chaetopteryx schmidi Botosaneanu, 1957b: C. papukensis was synonymised with $C$. schmidi by Malicky 2014:52.

According to Malicky (2014) the lateral fingers of the aedeagus are small like in C. schmidi, therefore he has synonymized $C$. papukensis with C. schmidi. Probably he has identified specimens of $C$. balcanica sp. nov. from the Đerdap Mts. (Serbia) as $C$. schmidi and his conclusion was based on this comparison. We have examined 17 populations of $C$. papukensis from the Papuk and the nearby Krndija Mts. (Croatia) with 5 populations of $C$. schmidi from the locus typicus region in Romania. Their diverged trait matrices demonstrate clearly that the lateral processes on the aedeagus of $C$. papukensis are entirely dif ferent from the lateral processes of the $C$. schmidi 

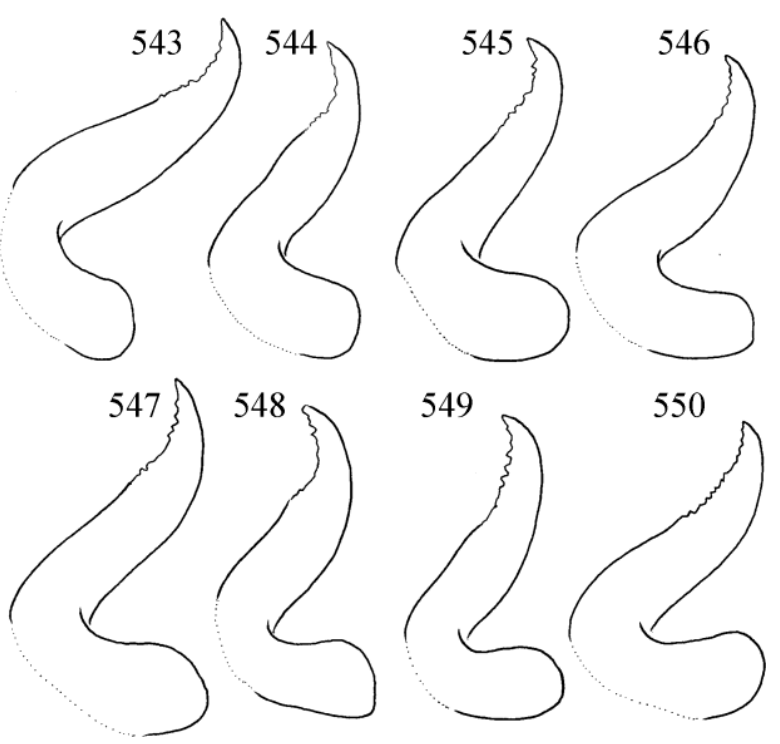

Figures 543-550. Chaetopteryx papukensis Oláh \& Szivák, 2012. Dorsal branch of the paraprocts in lateral view.

populations. The sclerotized or rigid basal tube of the lateral processes is almost perpendicular at $C$. schmidi and longitudinal at C. papukensis. The less rigid posterior part of the lateral process exhibits some range of variation, but much longer at $C$. papukensis. As it was detailed in the original species description the paramere shaft is triangular, not digitate; the stout primary spine is mesad turning at $C$. papukensis, not straight. It is also well visible from the trait matrices, that the primary spine is less stout and much longer at $C$. papukensis.

As also emphasized in the original species description the curvature of the paraproct hook is very different in the two species. Their diverged trait matrices indicate the hook formation is longer and less turned anterad in C. papukensis compared to $C$. schmidi. These divergences in paraproct hook formation are confirmed by the coevolution of the female anal tubes. The anal tube receives the inserted anchoring hooks during copulation. The abbreviated hook formation is accompanied by shorter anal tube at $C$. schmidi. These consistent and stable fine structure divergences look perhaps small under low magnifycation or for human scale, but are large and functioning as reproductive barrier on caddisfly scale.
In our routine taxonomical practice usually we examine specimens separately under microscope. The small divergences may seem not conspicuous enough especially under low magnification or with cursory examination. However if we put together the fine structure diagrammatic drawings of several specimens from several population into a trait matrix the differences are striking! Here we reinstate the specific status of Chaetopteryx papukensis Oláh \& Szivák, 2012.

\section{Chaetopteryx psunjensis Oláh, sp. nov.}

(Figures 571-573, 574-575)

Chaetopteryx papukensis Oláh \& Szivák, 2012 in Oláh et al. 2012: 62 (partim). Misidentification!

Diagnosis. This new species has elongated dorsal branch of the paraproct with blunt apex. The lateral process of the aedeagus is enforces by a short double S-shaped ridge connected directly to the basal sclerotized tube of the lateral process. Such a sclerotized basal tube has been evolved in C. kamnikensis of the C. rugulosa subgroup. This unique structural modification of the simple sclerotized cylinder of the aedeagus is an adaptive product of the sexual selection together with the modification of the apical hook formation on the paraproct. The dorsal profile difference in the female anal tube between the allotype and paratype is resulted probably by the long coupling pressure of anchoring paraproctal apical hook on the internal sclerites, as anchor substrate during copulation. During copulation the hook pair is inserted into the membranous anterior margin of the internal sclerite inside the anal tube exerting pressure to move the entire internal sclerite backward. The hook and sclerite interlocking keeps the male and female together for several days may move the internal sclerite backward exposing the bilobed very sclerotized apical part more free as well as slendering and elongating the entire anal tube accordingly.

Material examined. Holotype. Croatia, Sumetlica Strmac,

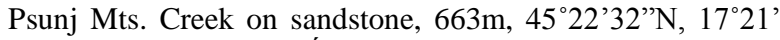
40”'E, 23.X.2012, leg. Á. Uherkovich (1ठ̄, OPC). Allotype. Croatia, Sumetlica Strmac, Psunj Mts. Small creek on crystalline rock, 722m, 45 22'43”N, $17^{\circ} 22^{\prime}$ '04'E, 23.X.2012, leg. Á. Uherkovich (1 female, OPC). Paratype. Same as allotype (1 female, OPC). 

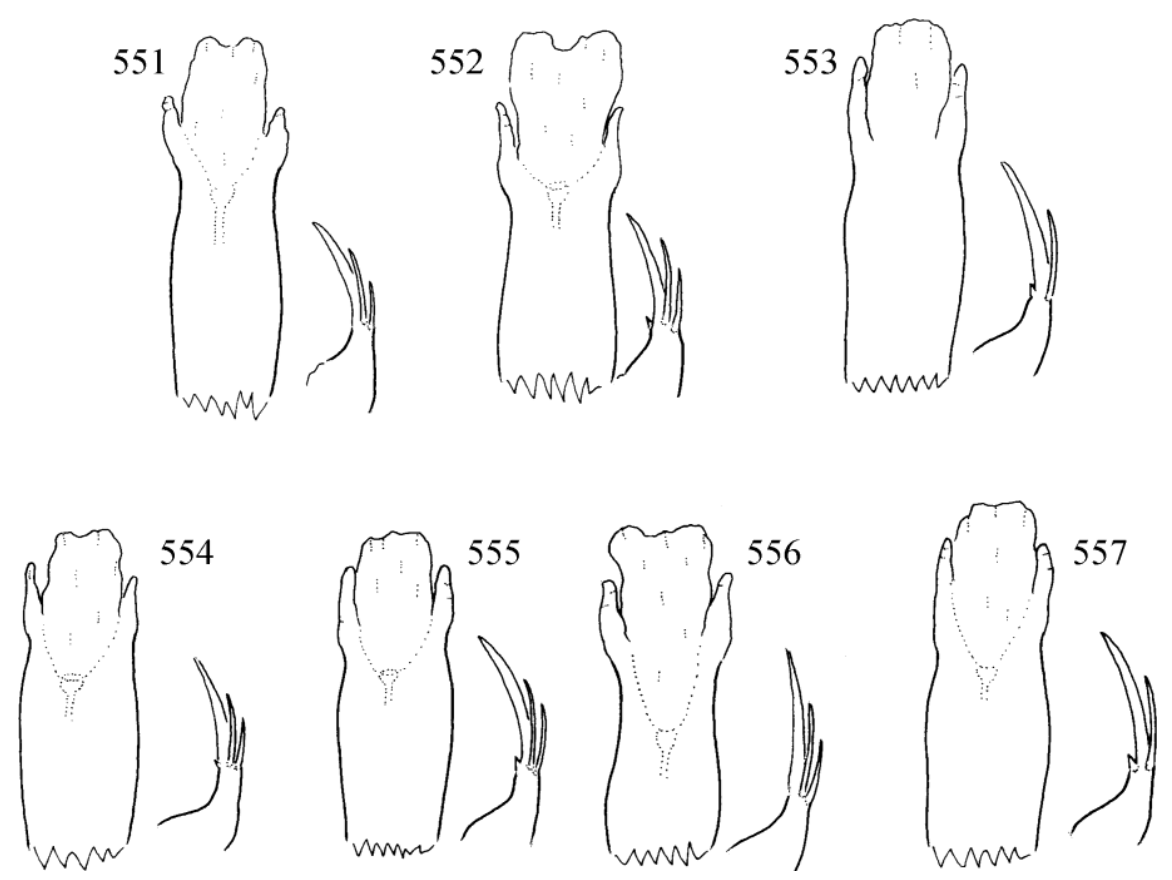

Figures 551-557. Chaetopteryx papukensis Oláh \& Szivák 2012. Aedeagus and paramere in dorsal view.

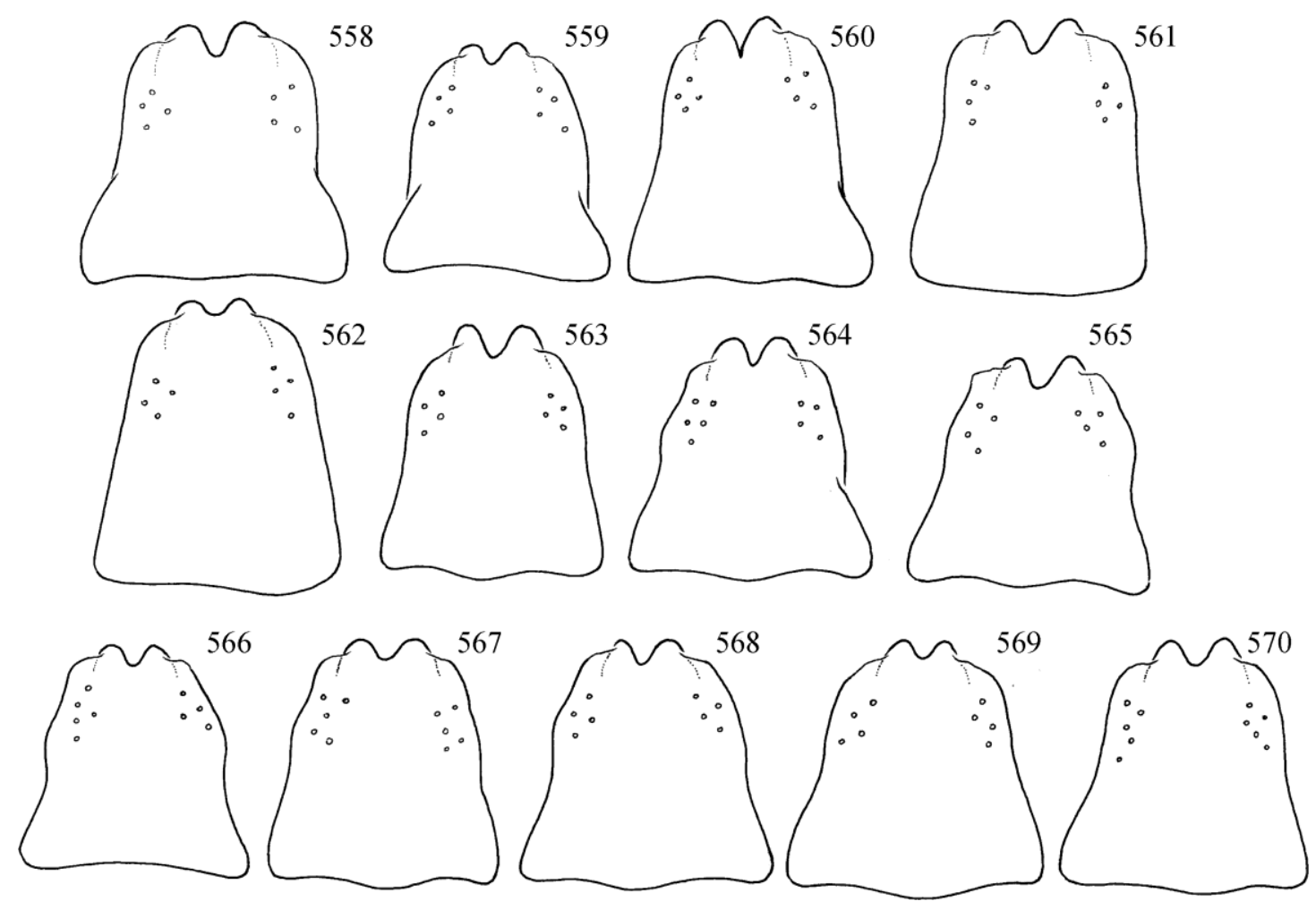

Figures 558-570. Chaetopteryx papukensis Oláh \& Szivák, 2012. Female anal tubes in dorsal view 


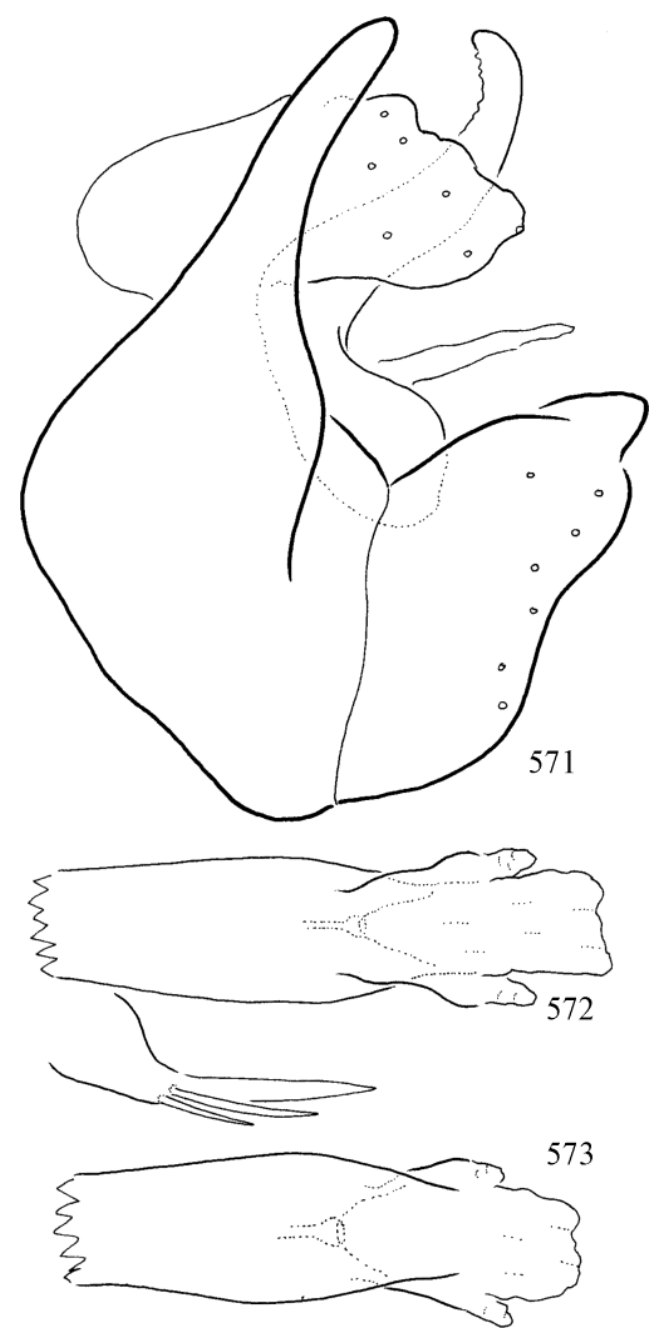

Figures 571-573. Chaetopteryx psunjensis sp. nov. $571=$ male genitalia in lateral view, $572=$ aedeagus and paramere in dorsal view, $573=$ aedeagus in ventral view.

Description. Male (in alcohol). Light brown small animal with light body appendages and with yellowish-testaceous wings. Anterior wing with rounded apex and with very long erect spine-like setae present both on the membrane and on the veins; setae on the veins usually stronger. Tibial spur formula is 033 . Forewing length is $7 \mathrm{~mm}$.

Male genitalia. Posterodorsal spinate area of vestitural noncellular microtrichiae on segment VIII and its mesal light band well developed. Segment IX with short, bridle-like dorsum and longer ventrum; this short bridle-like tergite IX of the fused segment IX is rather extending downward; the anterior margin of segment IX rounded

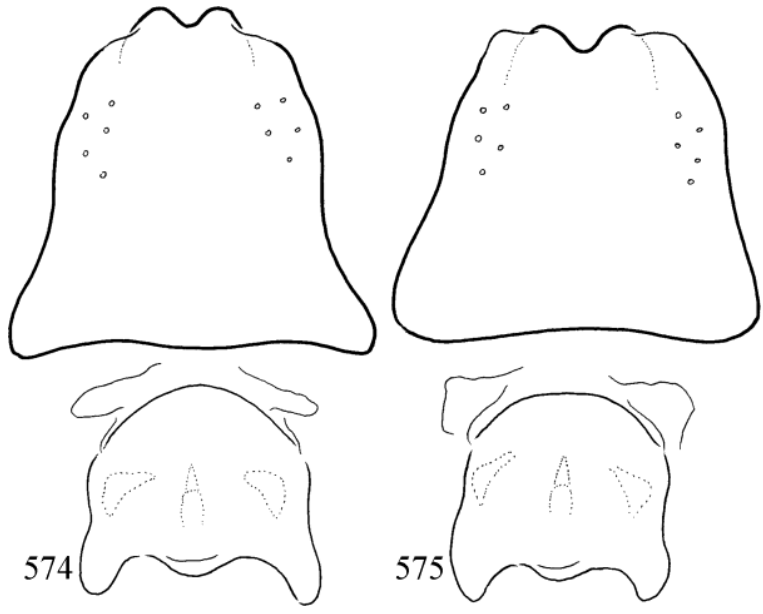

Figures 574-575. Chaetopteryx psunjensis sp. nov. Female anal tube and dorsal vaginal sclerite complex in dorsal view.

convex with long antecosta; posterior margin concave, midlateral sclerotized angle of tergite IX pronounced, extended downward. The pouch-like concavity of segment $\mathrm{X}$ long. Cerci are high with irregular posterior margin. Apical hook of the paraproctal complex elongated with blunt apex. Membranous subanal lobe long. Gonopods short with apical flap developed and turning mesad resulting in a single short narrowing apex in lateral view. Phallic organ composed of short rim-like phallic apodeme, short tube of phallotheca, short endotheca, well-developed aedeagus and medium long slightly triangular parameres in dorsal view; paramere with stout curving primary spine (enforced terminal shaft formation), 2 secondary spines and 1 tertiary spines of modified setae; aedeagus supplied with a pair of digitate lateral processes directed oblique upward, the very basal tube of the lateral process sclerotized and membranous after; ejaculatory duct ending with sclerotized gonopore opening into a large trilobed endophallic membranous structure withdrawn deep into the sclerotized cylinder. The sclerotized basal tube of aedeagus is enforced by a pair of simple, short and double S-shaped dorsal ridge.

Female genitalia. Anal tube formed by the fusion of tergite IX and $\mathrm{X}$ is medium long and broad, slightly narrowing posterad; apical margin forming rounded lateral lobes and mesal excision in dorsal view; this dorsal apical profile is created entirely by tergite $\mathrm{X}$, that is the internal sclerites, 
the remnant tergite $\mathrm{X}$ protruding, and taking part in the formation of the apical profile. Copulation may enlarge the extent of projection. Setose ventroapical lobes of tergite IX lacking. Supragenital plate of segment $X$ subtriangular in ventral view. Median lobe of the vulvar scale (lower vulvar lip) half long as the lateral lobes. Vaginal chamber medium sized reaching to the middle of sternite VIII. Vaginal sclerite pattern with rounded slightly mesad turning anterior apodemes.

Etymology. This new species was named after the mountain range where the type material was collected.
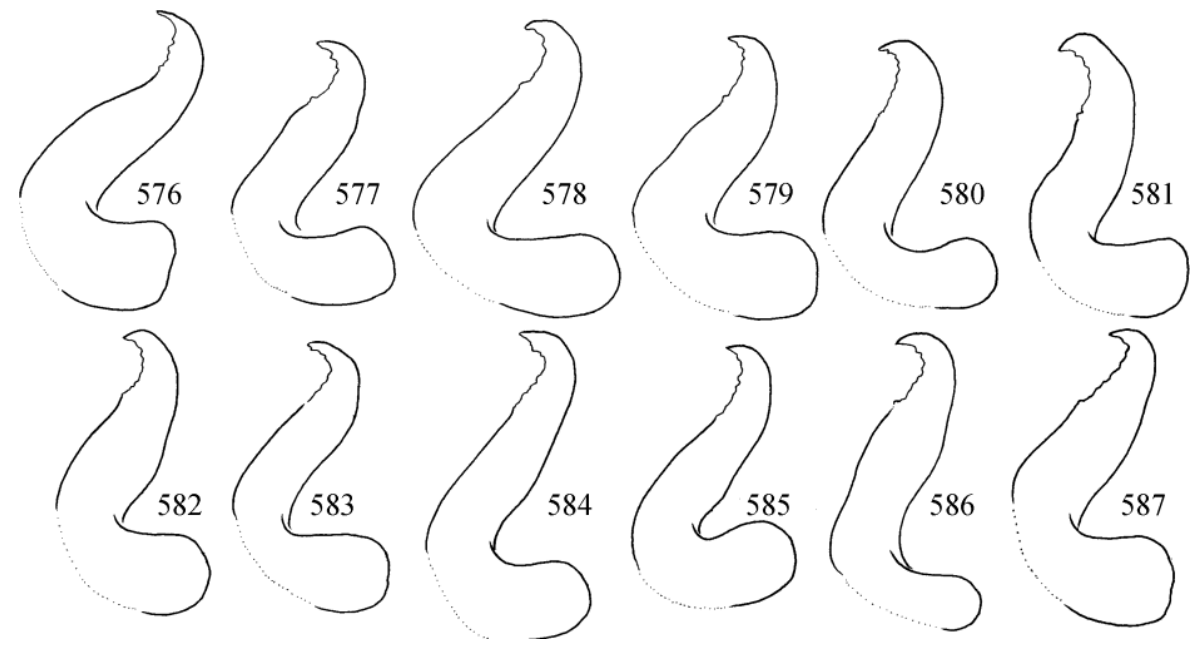

Figures 576-587. Chaetopteryx schmidi Botosaneanu, 1957. Dorsal branch of the paraprocts in lateral view.

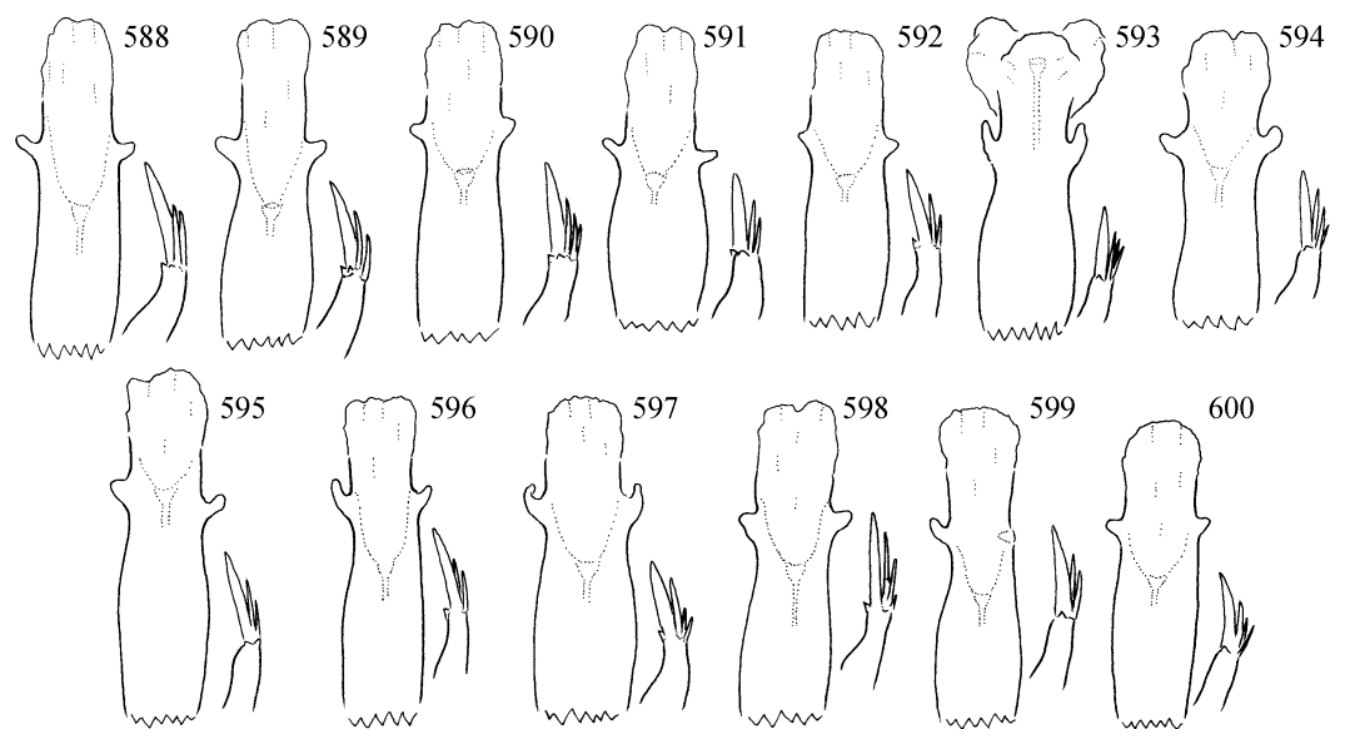

Figures 588-600. Chaetopteryx schmidi Botosaneanu, 1957. Aedeagus and paramere in dorsal view. 

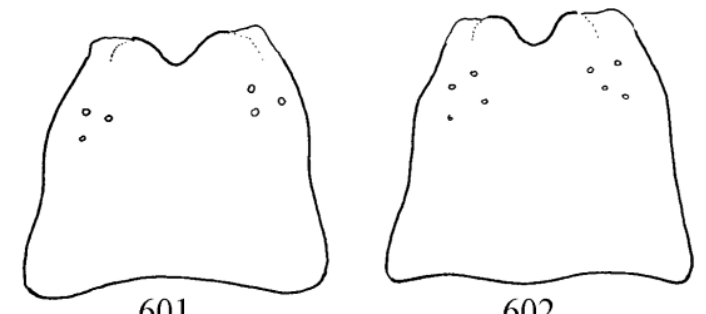

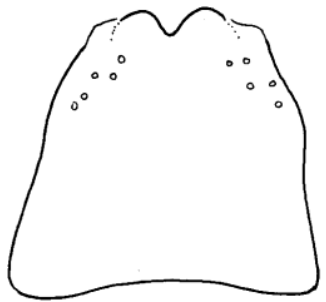

603

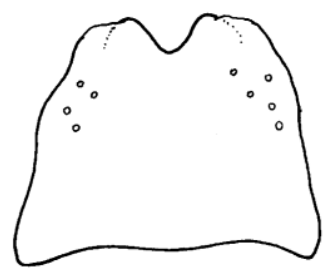

605

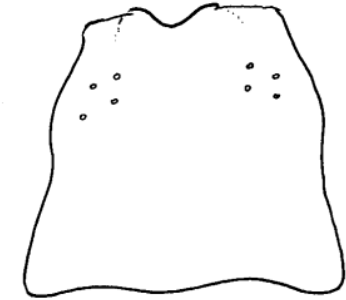

604

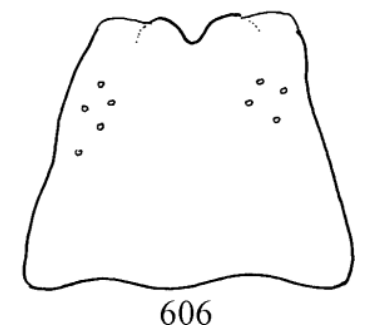

606
Figures 601-606. Chaetopteryx schmidi Botosaneanu, 1957. Female anal tubes in dorsal view

\section{Chaetopteryx tompa Oláh, sp. nov.}

(Figures 607-610)

Chaetopteryx schmidi Botosaneanu, 1957. Oláh et al. 2012: 62 (partim). Misidentification!

Diagnosis. This new species is close to C. balcanica sp. nov. but differs by having paramere shaft triangular, not digitate; primary spine short, stout and straight, not long and curved; dorsal branch of the paraproct, the apical hook formation is different, longer with blunt apex; female anal tube longer and not quadrangular. A more detailed examination on several specimens from more population is required to establish its relations.

Material examined. Holotype. Bosnia-Hercegovina, Skender, Vakuf, 820m, 19.X.1990, leg. B. Horvát \& I. Sivec, (1 male, OPC). Allotype same as holotype (1 female, OPC).

Description. Male (in alcohol). Light brown medium-sized animal with light body appendages and with yellowish-testaceous wings. Anterior wing with rounded apex and with very long erect spine-like setae present both on the membrane and on the veins; setae on the veins usually stronger. Tibial spur formula is 033. Forewing length is $8 \mathrm{~mm}$.

Male genitalia. Posterodorsal spinate area of vestitural noncellular microtrichiae on segment VIII and its mesal light band well developed. Segment IX with short, bridle-like dorsum and longer ventrum; anterior margin rounded convex with long antecosta; posterior margin concave, midlateral sclerotized angle of tergite IX pronounced. The pouch-like concavity of segment X long. Cerci high. Apical hook of the paraproctal complex with pointed apex, with shallow curvature. Membranous subanal lobe long. Gonopods short with apical flap developed and turning mesad resulting in a single short narrowing lobe in lateral view. Phallic organ composed of short rim-like phallic apodeme, short tube of phallotheca, short endotheca, well-developed aedeagus and medium long triangular parameres; paramere with short, stout and straight primary spine (enforced terminal shaft formation), 2 secondary spines of modified setae; simple sclerotized cylinder of the aedeagus slightly bellied in dorsal view; supplied with a pair of short digitate lateral processes probably retractable, only the very basal ring of the lateral process is sclerotized and membranous after; ejaculatory duct ending with sclerotized gonopore opening into a large trilobed endophallic membranous structure. The sclerotized basal tube of aedeagus is simple cylinder without any enforced supporting ridges or flanges.

Female genitalia. Anal tube formed by the fusion of tergite IX and $\mathrm{X}$ is long and narrowing apicad in dorsal view; apical margin with Vshaped excision. The internal sclerite of the anal tube, the vestigial tergite $\mathrm{X}$ is not protruded posterad. Setose ventroapical lobes of tergite IX lacking. Supragenital plate of segment X subtriangular in ventral view. Median lobe of the vulvar scale (lower vulvar lip) half long as the lateral lobes. Vaginal chamber medium sized reaching to the middle of sternite VIII. Vaginal sclerite pattern with rounded mesad turning anterior apodemes.

Etymology. tompa from "tompa" blunt in Hungarian, refers to the blunt apex of the hook formation on the dorsal branch of the paraproct. 


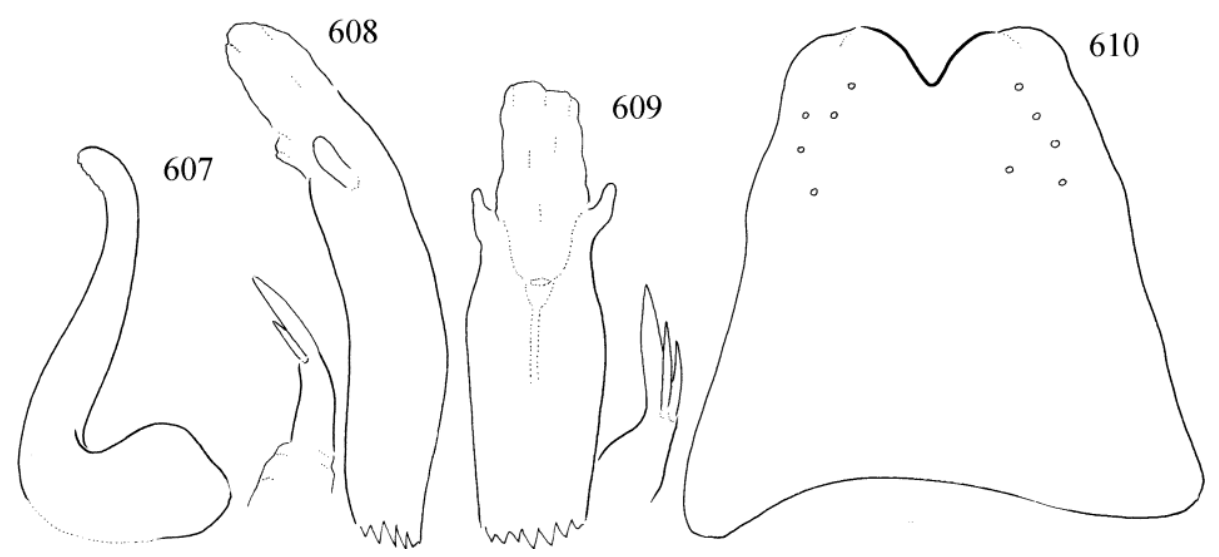

Figures 607-610. Chaetopteryx tompa Oláh, sp. nov. 607 = dorsal branch of the holotype paraproct in lateral view, $608=$ aedeagus and paramere in lateral view, $609=$ aedeagus and paramere in dorsal view, $700=$ female anal tube in dorsal view.

\section{Chaetopteryx rugulosa species subgroup}

Chaetopteryx rugulosa species subgroup. Oláh et al., 2012: 63

\section{Chaetopteryx noricum species cluster}

Chaetopteryx noricum species cluster. Oláh et al., 2012: 63.

\section{Chaetopteryx pohorjensis Oláh \& Urbanic, 2012 and Chaetopteryx noricum Malicky, 1976}

(Figures 611-613, 614-616)

Malicky (2014) has not synonymized C. pohorjensis with $C$. noricum, but his position was uncertain and confusing, moreover he maintained without any explanation that $C$. noricum is a subspecies of $C$. rugulosa and $C$. pohorjensis does certainly not merit a specific name. Above we have summarised the older divergences in the $C$. rugulosa species group forming the subgroup and species cluster structures as was detailed earlier (Oláh et al. 2012). Subgroups and cluster structures are differentiated by neutral traits of periphallic organs of older divergences, by gross phallic structures and by specific divergences in the speciation trait of the lateral process on the aedeagus. C. noricum species cluster is diverged from C. rugulosa species cluster by having entirely diferent aedeagus with large inflated and rigid flexible lateral processes enforced and supported by a pair of heavily sclerotized ventral flanges. At higher magnification this type of aedeagus seems clearly diverged far from the species of the $C$. rugulosa cluster. This magnitude of shape divergence may realize dramatic changes in copulatory processes and mating signals. C. pohorjensis compared to $C$. noricum, as detailed in the original species description, has well diverged paramere shaft and paramere spine pattern, very enlarged tube of lateral processes supported by a short, heavily sclerotized pair of ventral flanges. Moreover the ventral lip of the female anal tube of

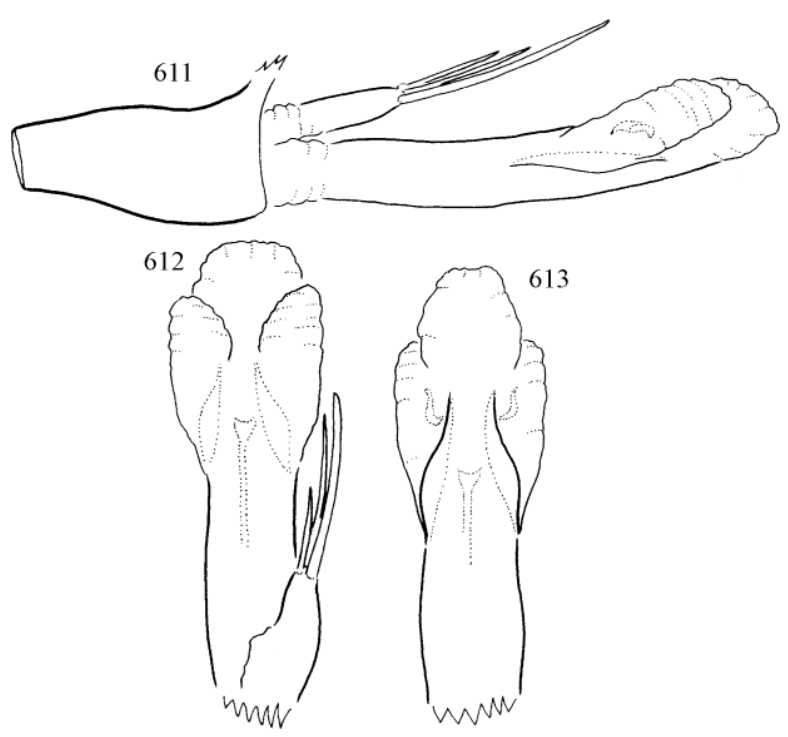

Figures 611-613. Chaetopteryx pohorensis Oláh \& Urbanic, $2012,701=$ phallic organ in lateral view, $702=$ aedeagus and paramere in dorsal view, $703=$ aedeagus in ventral view. 


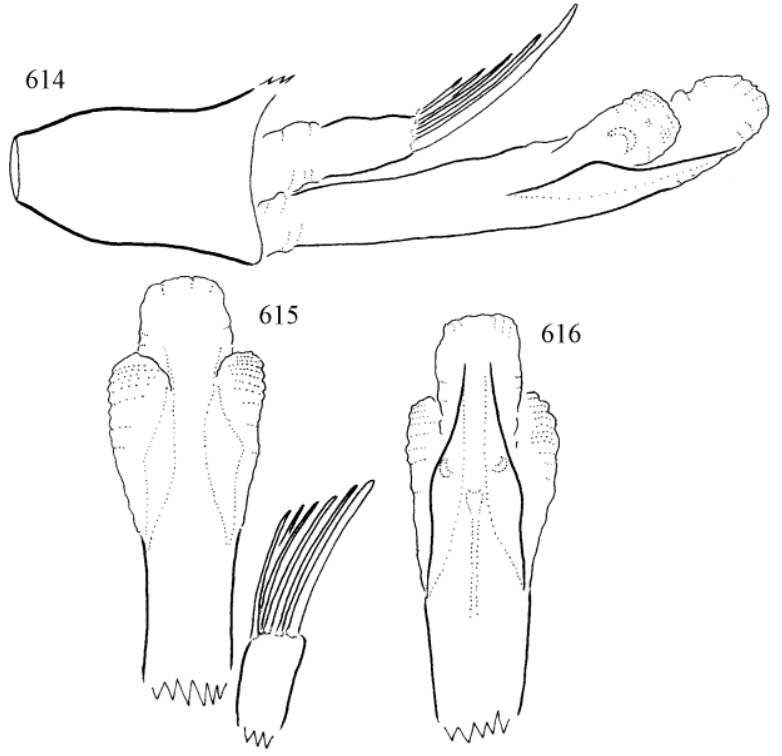

Figures 614-616. Chaetopteryx noricum Malicky, 1976. 704 $=$ phallic organ in lateral view, $705=$ aedeagus and paramere in dorsal view, $706=$ aedeagus in ventral view.

C. pohorjensis is short, not long. The female of $C$. noricum has diverged significantly from all members of the entire species group by having elongated ventral lip on female anal tube. It is unique for the entire species group that the ventral lip is longer than the dorsal lip. This old divergence of the ventral lip is stable and well visible under lower magnification. The elongated ventral lip of C. noricum is highly sclerotized, as usual in all the females of the C. rugulosa species group, its distinct divergence from $C$. pohorjensis is easily recognised at first glance.

\section{Chaetopteryx rugulosa species cluster}

Chaetopteryx rugulosa species cluster. Oláh et al., 2012: 65.

\section{Chaetopteryx kamnikensis Oláh \& Urbanic, 2012}

Chaetopteryx kamnikensis Oláh \& Urbanic, 2012: 66-68 in Oláh et al. 2012.

Malicky (2014) has not synonymised this species, but his position was vague or even confused. Here we do not detail our original description and diagnosis, simply we repeat that the divergence of paramere spine pattern and the female anal tube distinguish this species both from $C$. prealpensis and from C. rugulosa. Moreover the lateral process is not platiform- shaped, the platiform process is characteristic for $C$. prealpensis. It is digitiform like the lateral process of $C$. rugulosa, but with the evolution of the very specific sclerotized basal tube lacking at $C$. rugulosa. This is why the oblique direction of the process is fixed at $C$. kamnikensis does not depend on the erection state of the endophallus. Without this supporting sclerotized basal tube on the lateral process of $C$. rugulosa, the position, or oblique direction of the lateral process depends on the erection state of the endophallus.

\section{Chaetopteryx prealpensis Oláh, 2012 stat. restit.}

(Figures 617-649)

Chaetopteryx prealpensis Oláh, 2012: 68-71, in Oláh et al. 2012.

Chaetopteryx rugulosa Kolenati, 1848: Synonymised by Malicky 2014: 52.

This species with its very large distributional area is the putative ancestral species of the $C$. rugulosa species cluster. Malicky (2014) has synonymized $C$. prealpensis with $C$. rugulosa, declaring that the lateral processes are variable in their length, only slightly sclerotized, and may easily be deformed during maceration. In 2014 we have resampled three more populations in the Köszeg Mts. and one population in the Örség NP in Hungary and re-examined with care and sophistication our old materials from Croatia, Slovenia, and Austria (population details in Oláh et al. 2012). We have found, as earlier, the opposite what Malicky wrote. The lateral process is very stable and consistently structured in so many populations from the entire large distributional area. It is sagittaly flat, not digitiform like at $C$. rugulosa or gemmiform like at $C$. zalaensis, it is vertically plate-shaped or platiform (a terminus technicus standardization following the terms of digitiform and gemmiform). It is natural that there are individual variations, no two animals are identical even in the diverged adaptive traits, but the platiform shape is in the range of the basic 

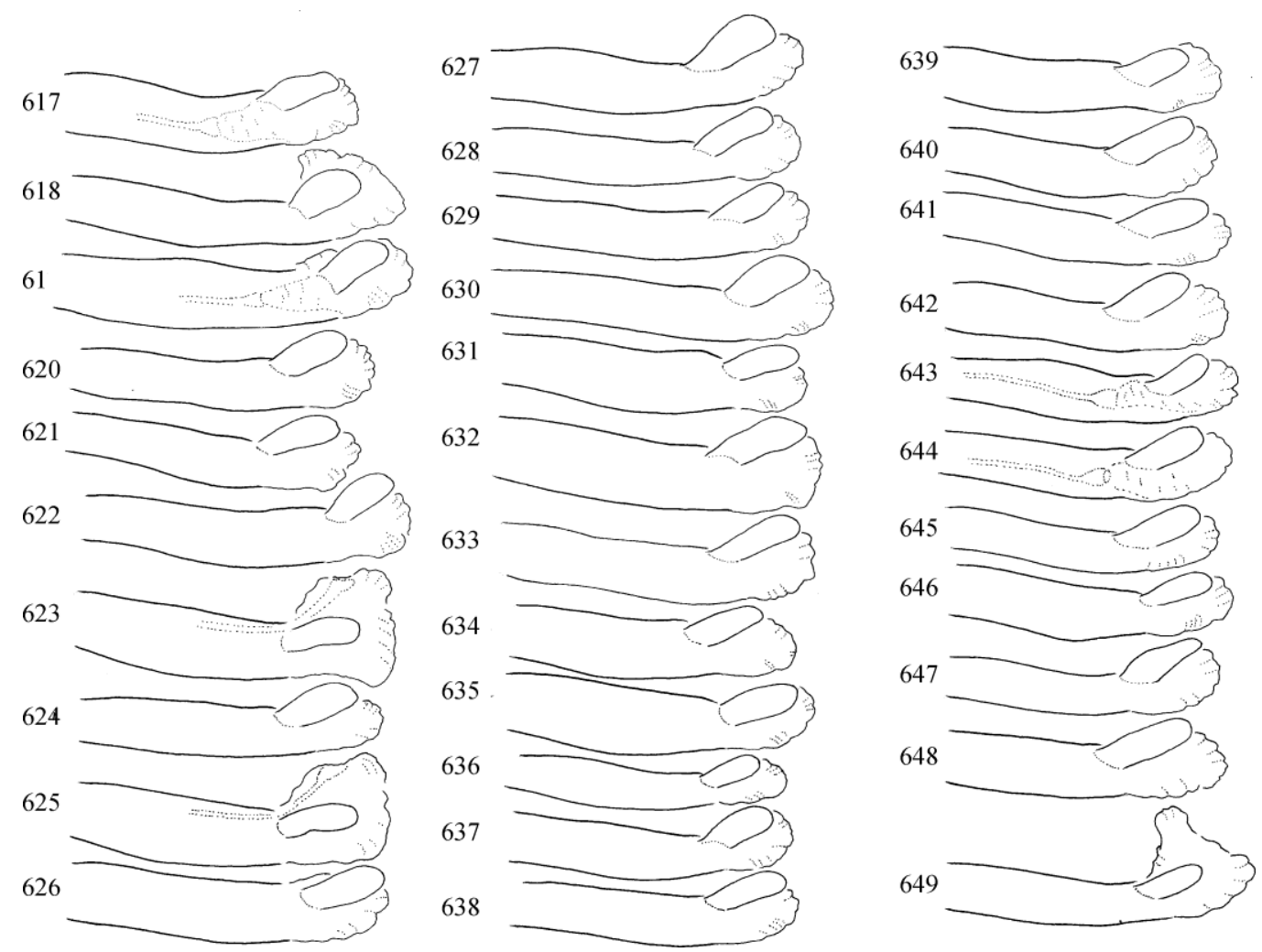

Figures 617-649. Chaetopteryx prealpensis Oláh, 2012. Aedeagus in lateral view. 617-619= Croatia, Medvednica Mts., 620622 = Croatia, Zumberacka Mts., 623-625 = Slovenia, Pohorje Mts., 626-628 = Slovenia, Kamnik Mts., 629-632 = Slovenia, Smrekovec Mts., 633-634 = Austria, Packalpe Mts., 635-638= Austria, Koralpe Mts., 639-644 = Hungary, Kőszeg Mts., 645-649 = Hungary, Apátistvánfalva.

architecture. The process is not much sclerotized, but seems rather rigid. At least if we compare the lateral view of the process drawn in the diverged trait matrix it is well visible that the different specimens have differently erected endophallus, but the shape of the lateral process is similar, stable. The divergence of the lateral process represents the speciation trait evolved in sexual selection processes and manisfests itself as a possible reproductive barrier in prezygotic phase realized by cryptic female choice, sperm competition or by other unknown mechanisms. Based on this divergence we reinstate the incipient phylogenetic species state of Chaetopteryx prealpensis.

New material examined. Hungary, Köszeg Mts. Stájer Házak, Ciklámen spring, 24.X.2014, leg. M. Máté, J. Oláh \& M. Oláh (14 males, 9 females; OPC; 10 males, 4 females; CSNMB). Köszeg Mts. Sáros spring, 24.X.2014, leg. M. Máté, J. Oláh \& M. Oláh (4 males, 3 females; OPC). Köszeg Mts. Hörmann Spring, 24.X.2014, leg. M. Máté, J. Oláh \&
M. Oláh (3 males, 2 females; OPC). Örség National Park, Apátistvánfalva, N 46 53 ' 44.63" E $16^{\circ} 15$ ' 34.80", 30.X. 2014, leg. M. Máté (5 males, 3 females, OPC).

\section{Chaetopteryx rugulosa Kolenati, 1848}

(Figures 650-671, 672-693)

Chaetopteryx rugulosa Kolenati, 1848: 73.

The holotype of $C$. rugulosa has slender digitiform lateral process (Malicky et al. 1986). In 2014 we have examined several populations newly sampled in side valleys of the main Stiftingtal valley near Graz near the headwater regions of the River Rába. In our new collection trial we have found a small $C$. rugulosa population also in the Örség NP in Hungary again in a small spring stream belonging to the system of River Rába. In diverged trait matrix we have presented diagrammatic lateral drawings of aedeagus with the lateral 

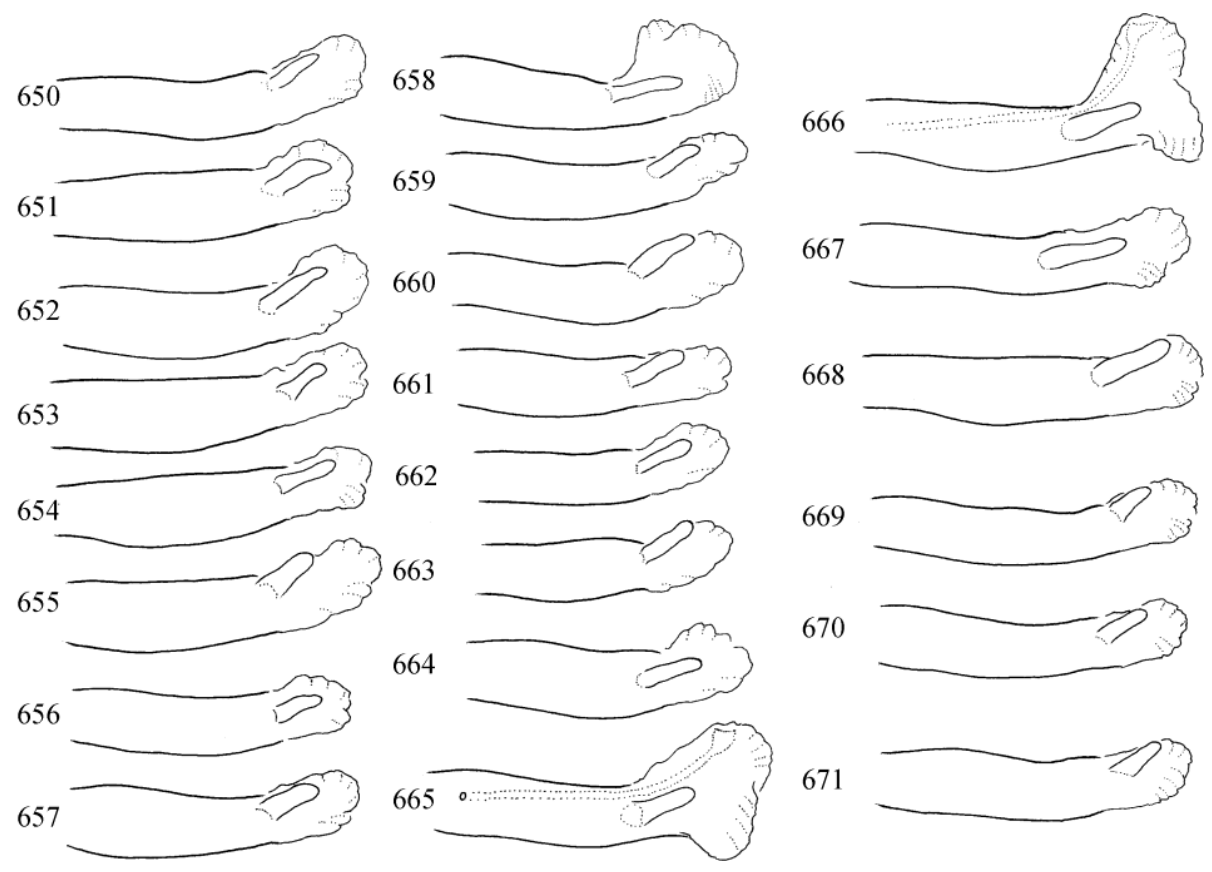

Figures 650-671. Chaetopteryx rugulosa Kolenati, 1848. Aedeagus in lateral view. 650-658 = Austria, Stiftingtal, 2006; 659-663 = Austria, Stiftingtal, 2014, 664-665 = Austria, Pflenzengreith, 666-668 = Austria, Gleinaple, 669-671 = Hungary, Kétvölgy.

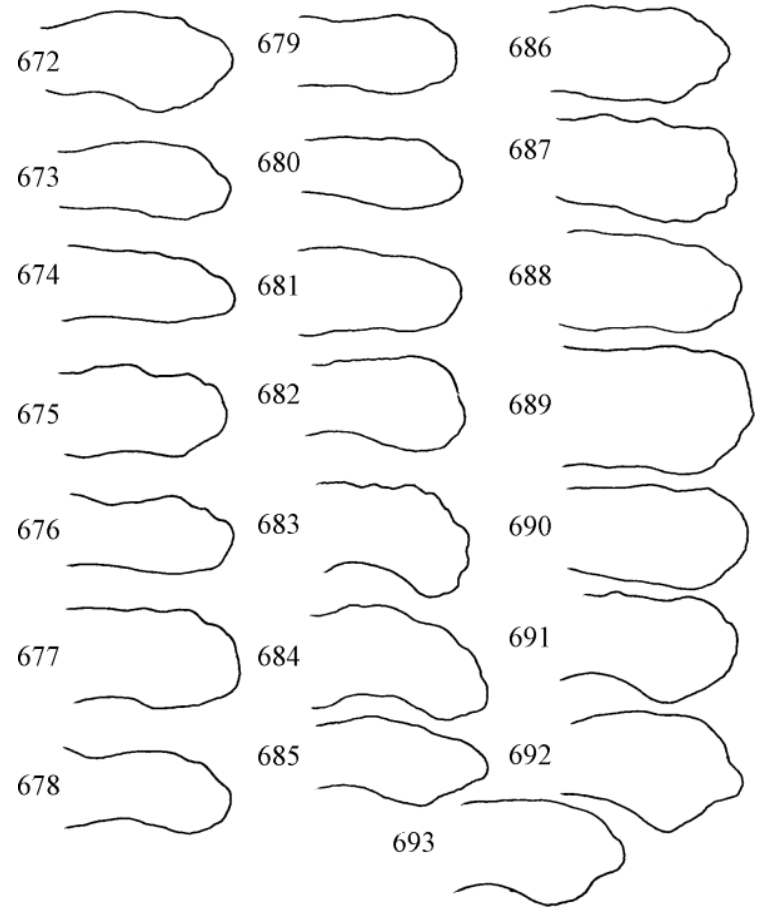

Figures 672-693. Chaetopteryx rugulosa Kolenati, 1848. Cerci in lateral view. 672-679 = Austria, Stiftingtal, 2006; 680-685 = Austria, Stiftingtal, 2014, 686-687 = Austria Pflenzengreith, 688-690 = Austria, Gleinaple, 691-693 = Hungary, Kétvölgy. process and with the endophallus of 22 specimens representing one Hungarian and 8 Austrian populations. The finger-like lateral process of $C$. rugulosa is consistently different from the vertically flat, plate-shaped, platiform lateral process of $C$. prealpensis. Moreover the lateral process of $C$. prealpensis is double or even triple sized. Similarly to $C$. prealpensis the size of the lateral process has no significant relationship to the erection state of the endophallus.

Malicky (2014) emphasized that C. rugulosa was described from Dalmatia based on Kolenati's description: "Habitat in Dalmatia (STENZ!)". However, in an earlier paper Malicky et al. (1986) have questioned the reality of the habitat data of the insect dealer Stenz: "In der ersten Halfte des vorigen Jahrhunderts nahm man es mit der Etikettierung nicht so genau". We have sampled real Dalmatian costal area in right time and in right habitats several times, but we have not collected any specimens from the C. rugulosa species group. We have collected members of this species group in internal mountain ranges in Bosnia-Herzegovia and Serbia, but all belongs to the $C$. schmidi subgroup. No C. rugulosa or $C$. 
prealpensis live in Dalmatia or even nearby Dalmatia. The holotype is in good condition, aedeagus perfectly preserved, and all the specimens, with exactly the same lateral process, was collected in a very restricted area near around Graz, except the single Hungarian population just discovered.

New material examined. Austria, Graz, Stiftingtal stream tributaries, 25.X.2014, leg. M. Máté, D. Stradner, J. Oláh \& M. Oláh (12 male, 7 females; OPC, 5 males, 3 females; CSNMB). Hungary, Örség National Park, Kétvölgy, N 465' 12.41” E 16 ${ }^{\circ} 13^{\prime} 42.00 ”, 30 . X .2014$, leg. M. Máté (3 males, 3 females, OPC).

\section{Chaetopteryx zalaensis Oláh, 2012 stat. restit.}

(Figures 694-728, 729-859)
Chaetopteryx zalaensis Oláh, 2012: 71-73 in Oláh et al. 2012.

Chaetopteryx rugulosa Kolenati, 1848: Synonymised by Malicky 2014: 52.

Malicky (2014) has synonymized C. zalaensis having gemmiform lateral process with $C$. rugulosa having long digitiform lateral process, repeating again that the lateral process is variable. So we need to repeate again that according to the diverged trait matrices this speciation trait is consistently stable. Stability is a basic feature of adaptive structures evolved in the sexual selection processes. Naturally there is a variation range as a result of individuality principle, but this is far from the same type of variation ranges in $C$. rugulosa species.
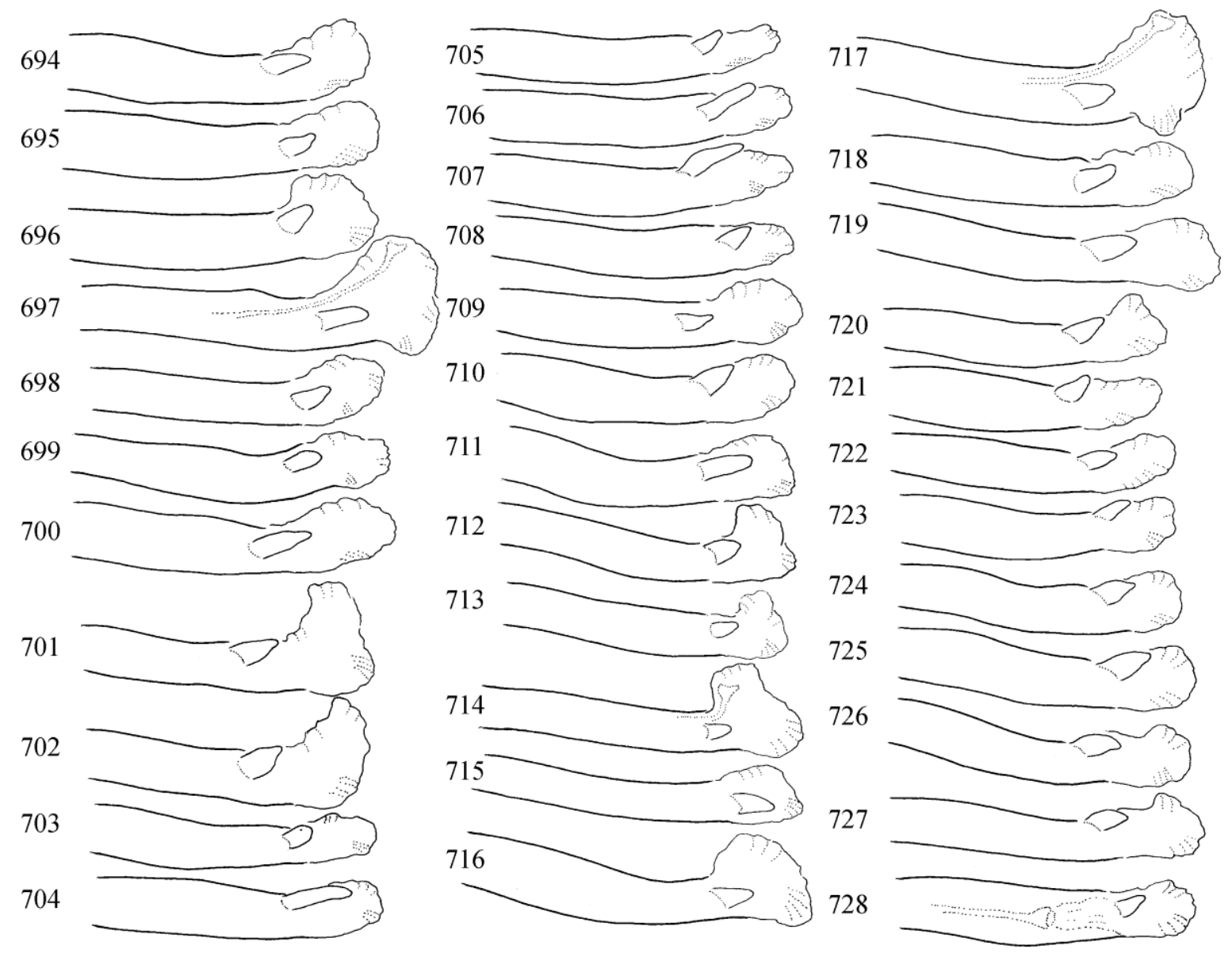

Figures 694-728. Chaetopteryx zalaensis Oláh, 2012. Aedeagus in lateral view. 694-700 = Hungary, Szőce, 1985; 701-710 = Hungary, Szőce, Biczó springs, 2014; 711-715 = Hungary, Szőce, Dam springs, 2014; 716-721 = Locus typicus: Hungary, Vadása spring, 2010; 722-728 = Locus Typicus: Hungary, Vadása spring, 2014. 


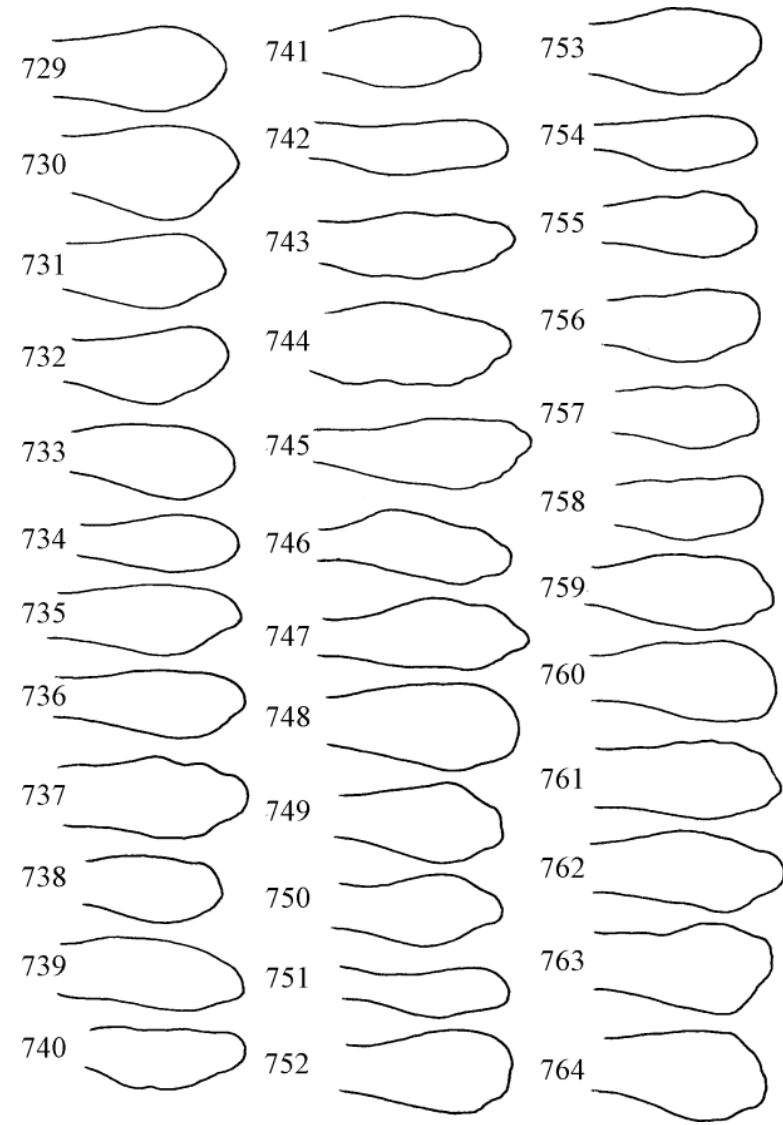

Figures 729-764. Chaetopteryx zalaensis Oláh, 2012. Cerci in lateral view. 729-734 = Hungary, Szöce, 1985; 735-741 = Hungary, Szőce, Biczó springs, 2014; 742-747 = Hungary, Szőce, Dam springs, 2014; 748-754 = Locus typicus: Hungary, Vadása spring, 2010; 755-764 = Locus Typicus: Hungary, Vadása spring, 2014.

However, like Malicky we have also found variation in the same population from Szöce stream (actually we have never collected $C$. zalaensis along the stream; they populate exclusively the side spring area, a very frequent habitat along the Szőce stream). We have compared 45 specimens collected in 2014 with 15 specimens collected by Uherkovich and Malicky in 1985 and found specimens with longer lateral processes, but in a very low ration. The population inhabiting the isolated Vadása spring area, the locus typicus of $C$. zalaensis is however not mixed; it is pure having not any specimens with finger-shaped lateral process, a character state of $C$. rugulosa. We have found no specimens with digitiform lateral process neither in the year of 2010 nor in the year of 2014.
However, the possibility of secondary contact zone between $C$. rugulosa and $C$. zalaensis is high. The system of River Zala is nearby to the system of River Rába. The River Zala has eroded parts of its valley into the gravel-pebble deposition of the former bed of River Rába.

Trait variability is higher within contact hybrid zone than in putatively pure populations. However, the possibility of primary sympatric speciation cannot be ruled out. Sympatric speciation is getting more and more attention as the only genuine process of the classical Darwinian natural selection.

In the original species description we have characterized cerci as low and stalked, not parallel-sided in contrast to C. rugulosa. It is well known that neutral traits of the periphallic organs like cerci and gonopods may vary in a wide range in $C$. rugulosa species group. Nevertheless we have found a clear basal constriction in the lateral shape of the cerci. Malicky (2014) questioned this shape, attributing to erroneous view of aspect. We are very much aware that the view or the aspect of examination is decisive in studies or drawings, especially in fine structure analysis. Therefore we prefer in our species' descriptions to use mostly the lateral view to draw structures, as we have found as the most stable aspect of examination. We compared cerci of $C$. zalaensis with $C$. rugulosa with careful attention to exact lateral aspect of the cerci. We focused on the lateral aspect of the cerci alone, not on the lateral aspect of the entire segment IX, because the position of cerci is frequently oblique. We have found shape divergence in the neutral cerci clearly detectable without any geometric morphometrics. This stalked shape is however a neutral trait, we have relied more upon the divergence of the adaptive speciation trait of the lateral process. Here we reinstate the specific status of the incipient phylogenetic species Chaetopteryx zalaensis.

New material examined. Hungary, Örség, Szőce stream, Eastern arm, Biczó springs, N 46 54 ' 16.60" E $16^{\circ} 34$ ' 42.36" 26.X.2014, leg. M. Máté, J. Oláh \& M. Oláh (36 males, 11 females; OPC). Örség, Szőce stream, Eastern arm, Biczó springs, N 46 54 ' 16.60" E $16^{\circ} 34$ ' 42.36" 23.XII.2014, leg. M. Máté, (2 males, 2 females; OPC). Örség, Hegyhát- 
szentjakab, Vadása-tó Spring, 18.X.2014, leg. M. Máté (4 males, 5 females; OPC, 5 males, 3 females; CSNMB). Örség, Hegyhátszentjakab, Vadása-tó Spring, N 46 52' 33.37” E 16³3' 06.13” 23.X.2014, leg. M. Máté, J. Oláh \& M. Oláh (6 males, 5 females; OPC). Örség, Szőce stream, Dam springs, 26.X.2014, leg. M. Máté, J. Oláh \& M. Oláh (7 males, 7 females; OPC). Örség, Szőce stream, spring at bridge, 26.X.2014, leg. M. Máté, J. Oláh \& M. Oláh (1 male, OPC).

\section{Chaetopteryx irenae species subgroup}

Chaetopteryx irenae species subgroup. Oláh et al., 2012:73

\section{Chaetopteryx giuliensis Oláh \& Kovács, 2012 and Chaetopteryx idriensis Oláh \& Urbanic, 2012}

(Figures 765-767)

Malicky (2014) has not synonymized these species, but his position was vague. We agree with him that these species are close to $C$. goricensis, but disagree with his other statements: "Except for the usual individual variability of the structures, the only difference is the number of the spines of the parameres, both having a small bunch of them". "C. goricensis normally has only one spine, but some specimens from the type locality Deskle have two". The number of spinelike modified apical setae nested on the tip of the parameres is stable in the examined 15 specimens of $C$. idriensis from three populations. There was a single specimen of $C$. giuliensis with left paramere having only two well-developed modified setae out of the 6 examined specimens from three populations. We have found also a specimen of $C$. goricensis from the type locality having 2 spines on the right paramere, but the second spine was very small vestigial.

As we have explained above in details the pattern of modified paramere setae are not a contemporary speciation trait in Chaetopteryx rugulosa species group, not diverging consistently on species level. They exhibit older divergences at subgroup level. Nevertheless C. goricensis has specific paramere spine pattern compared to $C$. giuliensis and C. idriensis. The three species have diverged significantly in several neutral traits, we do not list them here, and the differences are explained in details in the original species descriptions. In the $C$. rugulosa species group, as explained before, the speciation trait is the lateral process and the associated substructures on the aedeagus. The lateral processes are rather rigid and evolved into completely different shapes in the three species. C. idriensis with the smallest lateral process has no any sclerotized ridge or flange evolved to support the function of the lateral process. Simply it is not required; this small process may function perfectly without additional support of sclerotized structures. Here we can realise again that the divergence of the lateral process, the speciation trait in the building process of reproductive barriers is governed by sophisticated complex cooperation of several quantitative trait loci in concerted evolution. $C$. goricensis has larger lateral process and a small sclerotized ventral flange supporting its function. C. giuliensis evolved an extremely large lateral process in the form of a vertical subqudrangular plate. Its specific function is supported by a welldeveloped pair of ventral flange.

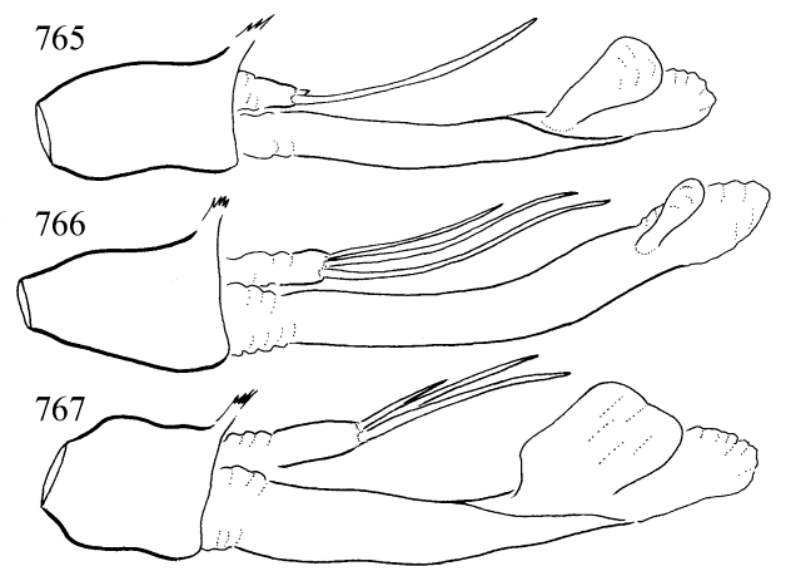

Figures 765-767. Phallic organs in lateral view. $765=C$. goricensis, $766=C$. idriensis, $767=C$. giuliensis .

\section{Psilopteryx Stein, 1874}

Type species: Chaetopteryx psorosa Kolenati (monotypic)

In the chaetopterygini tribe the erect and spinelike apomorphic setae are present both on the forewing membrane and on the forewing veins. Psilopteryx and Pseudopsilopteryx genera are 
unique by differing from all the other chaetopterygini genera having spine-like setae present only on the forewing veins, and lacking on the forewing membrane.

\section{Psilopteryx psorosa new species group}

In most limnephilids the dorsum of segment IX, housing the superanal genitalic complex (segment $\mathrm{X}$, cerci and paraproct), is usually short and reduced into a transversal band, bridle or keel. A unique apomorphic trait of the Psilopteryx psorosa species complex is the posterad elongated dorsal keel of segment IX. We prefer to call the longitudinal elongation of the transversal band of the short tergite IX as the dorsal keel of segment IX. The paraproct of the superanal genitalic complex is also modified into the apomorphic state of a huge elongated and lanceolated structure. The dorsal branch of the paraproct produced into a long triangular heavily sclerotized blade, its surface is densely granulated with short black outgrowths and the ventral branch is reduced and fused to the ventrobasal region of the modified dorsal branch. This enlarged, heavily sclerotized structure with particularly patterned surface of roughly assembled cobbles or teeth must have a dominant copulatory function of barriers in reproductive isolation. Cerci bilobed, its mesal lobe shifted below the elongated dorsal keel of segment IX. The dorsal keel, the mesal lobe of cerci, and the paraproct each are heavily sclerotized and black. Gonopods fused to segment IX, visible only a much reduced digital free process. Aedeagus enlarged with a pair of apical wings. Parameres are rod-shaped slightly upward arching and characterized by specific terminal dental pattern.

We have carried out a detailed examination of the fine structure on the dental pattern, as well as on the pattern of curvature, constriction and dilatation of the paramere rod and we have found similar types of individual deviations in all sibling species complexes with different and specific architecture. These types of deviations modify the individual patterns repeating similar alterations in various lineages, caused probably by similar epistatic genome mechanisms or by abrasion and damage effects in mating and copulatory pro- cesses. These deviation types diversify individuality but maintain the specific architecture of the parameres: (1) apical constriction or dilatation, (2) ventrosubapical constriction or dilatation, (3) median or basal dilatation, (4) apical upturning, (5) apical mesad turning, (6) dens proliferation, (7) dens reduction. Some of these alterations may represent the initial stage of divergence building up in isolation, like upturning frequency in the Vihorlat Mts. or mesad turning frequency in Beszczady Mts at the species of $P$. harmas sp. nov.

In a comprehensive study Mey \& Botosaneanu (1985) have elaborated the taxonomy of the variable Psilopteryx psorosa species. They have established six subspecies with the description of three new subspecies as well as erected two groups: $P$. carpathica group with long paraproct and P. psorosa group with short paraproct. Detailed study was conducted on the variability of genital substructures. The measured variation ranges were rather high both in male and female genital substructures: dorsal keel of segment IX, paraproct, gonopods, apical wings of aedeagus, external female genital substructures. Variation measured in these traits was significant both between and inside populations. This high morphological plasticity of almost all substructures has complicated the clear delimitation between taxa. Difficulties to separate between the established six subspecies by these variable characters were reported and clearly demonstrated by Szczęsny (1987) for North Carpathian populations.

During the last five years we have examine 94 populations of the six taxa in the Psilopteryx psorosa species complex collected from the entire distributional area. We found our fine structure analysis and speciation trait theory as promising tool to delimit taxa more reliably. In the previous studies the paramere was not considered, not examined, and not drawn at all. The simple upward arching rod-shaped parameres have diverse dental pattern on the apical and dorsosubapical region.We have cleared several specimens from all populations and prepared diverged trait matrices in order to demonstrate empirically both the infra- 
populational and intrapopulation variability of the parameres. All the examined taxa have species specific architecture of the apical dental pattern on the paramere head. At the same time almost every specimens have its own pattern in the range of the architecture evolved for each incipient species. The individual variability is more pronounced in populations collected from the putative contact zones of $P$. psorosa/P. bohemosaxonica, and $P$. transsylvanica/P. retezatica. Similar hybrid condition were found and discussed for neutral traits by Mey \& Botosaneanu (1985).

Compared to the paramere, the lateral shape of paraproct is more stable and more consistent in all the examined populations of each taxon. It seems that both the paramere and the highly modified paraproct participate, as an adaptive non-neutral trait, in the processes of reproductive isolation by sexual selection. Paramere divergence is probably less old with shallow split and incomplete lineage sorting. Paraproct divergence seems older and its split is deeper. The higher individual variability of paramere and the more stability of the paraproct might had various mechanisms to evolve: (1) hybridization along the contact zones; (2) incomplete lineage sorting; (3) different endogenous clines might meet and ovelap; (4) moving clines might be stabilized variously by physical bariers or by local adaptation; (5) coupling local adaptation with old intrinsic reproductive barriers; (6) preexisting barrier loci recruited to enhance ecological barierrs might explain the deeper coalescences of older trait split in paraproct phenotype (Abott et al. 2013); (7) evolution of reproductive barierrs through linkage disequilibrium between adaptive and assortative mating loci (Felsenstein, 1981).

We have distinguished three sibling species complexes in the Psilopteryx psorosa species group based on the diverse and stable paraproct as the product of older divergence with complete lineage sorting: $P$. bohemosaxonica, $P$. psorosa and $P$. carpathica. We have used the other adaptive, non-neutral trait the paramere, as a suplementary trait with higher intraspecific variability and incomplete lineage sorting, to differentiate inside the sibling species complexes. Neut- ral traits of dorsal keel, gonopods, and the lateral wing on the aedeagus as well as the female external genital substructures, not involved directly in building of the reproductive barierrs, all have exhibited very high variability due probably to migration, effective gene flow. It is well documented that introgression can easily erase the history of populations at most neutral markers.

Therefore it is advised to reconstruct the history of incipient species using the traits of barrier loci evolved by sexual selection. We are aware that it is desirable to connect phenotypes to nucleotide variation by studies of genomic divergence of genome scans, of quantitative trait loci mapping and of genome-wide association studies. However, this is a future challenge if at all the New Taxonomy and molecular genetics will really cooperate as independent sciences on biodiversity, or the old New Systematics continues to reign remaining on the routine pathway of neutral markers and barcoding. Fortunately, in caddisfly research we might capitalize the resolving power of the discovered speciation traits of paraprocts and parameres.

\section{Psilopteryx bohemosaxonica new sibling species complex}

This sibling incipient species complex is characterized by short dorsal branch of paraproct. The length of dorsal branch was established by measuring the ventral length from the tip to the reduced vestigial ventral branch of the paraproct. This vestigium is detectable in lateral view on the ventrobasal region of the paraproct as a sclerotized, but less pigmented therefore lighter, small triangle with smooth surface, without the black granulation so much characterizing the dorsal branches. The length of the dorsal branch of the paraproct is almost identical at all of the three species. The paraproct has short and high subtriangular shape with some trend of variation between species. The dental diversity on the paramere head evolved into significantly diverged pattern at the three species. The dental pattern of $P$. bohemosaxonica, the putative ancestral species of the complex, modified in $P$. gutinensis and simplified in $P$. javorensis sp. nov. The species 
complex of $P$. bohemosaxonica is distributed mostly in the mountain ranges of lower elevations from the Ore Mts to the Gutin Mts. However, its exact distributional area is not known due to putative extensive and complicated hybrid zones with $P$. psorosa. Three species belongs to this complex: $P$. bohemosaxonica, $P$. gutinensis, $P$. javorensis sp. nov.

\section{Psilopteryx bohemosaxonica Mey \& Botosaneanu, 1985 stat. nov.}

(Figures 768-806, 807-853)

Psilopteryx psorosa bohemosaxonica Mey \& Botosaneanu, 1985: 120.

Diagnosis. This sibling incipient species is chracterized by short dorsal branch of paraproct. The short and high subtriangular paraproct is rather stable. Some populations in the Gorce Mts and the Babia Gora Mts have some sign of paraproct elongation probably as a result of having contact zone or very complex and irregular moving cline with $P$. psorosa. The curvature of the paramere rod is shallow. The dental pattern on the paramere head is rather elaborated and packed with apical and dorsosubapical teeth. P. bohemosaxonica is distributed from the Ore Mts (Erzgebirge) to the Pol'ana Mts.

Material examined. Holotype and Allotype. Czech Republic, Erzgebirge, S of Cinovec, 12.X.1982, leg. W. Mey (MFN). Austria, Superior, Schwarzenberg, 28.X.1987, leg H. Malicky (2 males, 2 females; OPC). Czech Republic, Southern Bohemia, Sumava Mts. inlet of Laka lake N49 06'30" E13 ${ }^{\circ} 19^{\prime} 34 ", \quad 1090 \mathrm{~m}, 30$. IX. 2010, leg. J. Bojkova (9 males, 4 females, NMPC). Southern Bohemia, Sumava Mts. inlet of Cerné jezero lake, N49 10 '44" E1310'51", 1030m, 11. X. 2007, leg. P. Chvojka (5 males, 3 females, OPC). Southern Bohemia, Sumava Mts. left tributary of Teplá Vltava river below source, N48 $58^{\prime} 44^{\prime}$ " E1333'45”, 1160m, 16. X. 1992, leg. P. Chvojka (3 males, 1 female, NMPC). Northern Bohemia, Jizerské hory Mts. Ztraceny potok brook below Smrk Mt. N50 53'44", E15¹4'49”, 680m, 11.X.1989, leg. J. Preisler \& P. Vonicka ( 2 males, 3 females; NMPC). Northern Bohemia, Jizerské hory Mts. left tributary of Sous reservoir. N50 47'40" E15 19'22”, 800m, 4.XI.1989, leg. P. Chvojka (23 males, 16 females; NMPC). Northern Bohemia, Krkonose (Giant Mts.), left tributary of Labe (Elbe) river below Labsky vodopad waterfall, N5046'03" E1533'12", 1100m, 11.X.1992, leg. P. Chvojka (6 males, 5 females; NMPC). Eastern Bohemia, Orlické hory Mts., brook, Zidovsky kout SW Orlické Záhori,

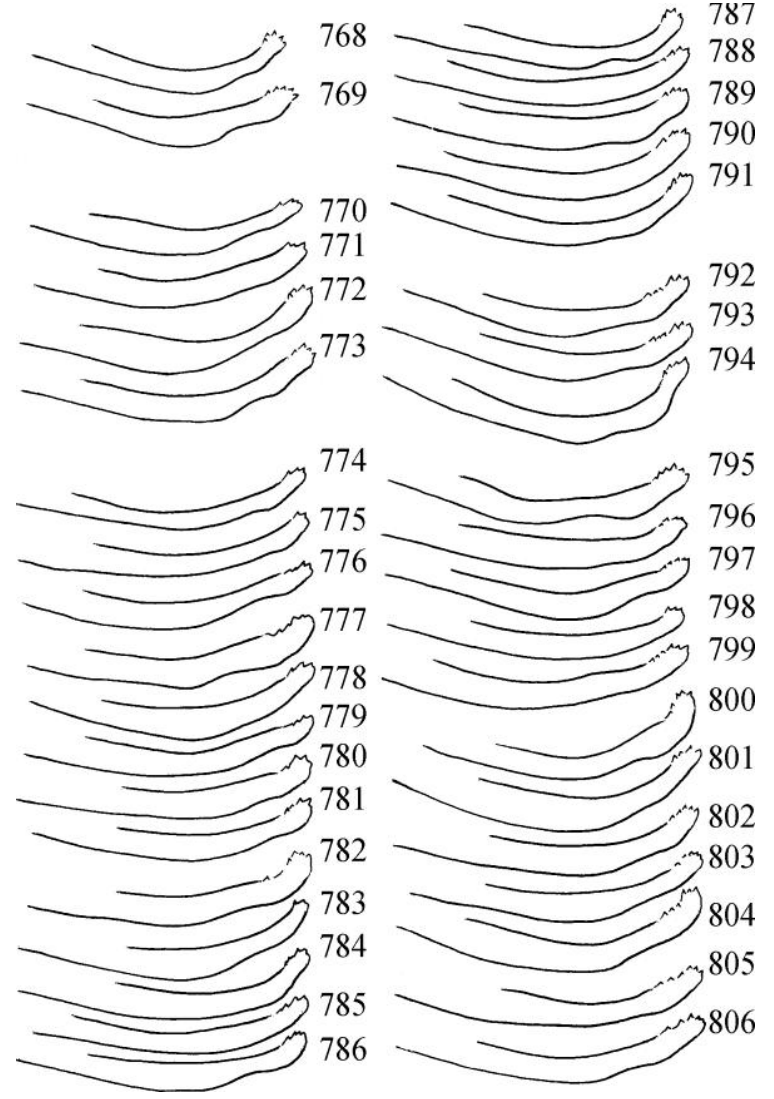

Figures 768-806. Psilopteryx bohemosaxonica Mey \& Botosaneanu, 1985. 768-769 = Austria, Schwarzenberg, 770 773 = Germany, Böhmerwald, 774-781 = Czech Republic, Orlické hory Mts., 782-786 = Czech Republic, Sumava Mts., 787-791 = Czech Republic, Jizerské hory Mts., 792-794 = Poland, Gorce Mts., 795-799 = Poland, Babia Gora Mts., 800-804 = Poland, High Tatra Mts. Rostoka; 805-806 = Slovakia, Poliana Mts.

N50'16'16" E16 27'03", 775-870m, 27.X.1994, leg. P. Chvojka (18 males, 12 females; NMPC). Eastern Bohemia, Orlické hory Mts., brook, NPR Bukacka reserve NW of Serlich Mt., N50²0’06” E16²2’31”, 880-970m, 26.X.1994, leg. P. Chvojka (158 males, 4 females; NMPC). Eastern Bohemia, Orlické hory Mts., brook S of Serlich Mt., N50 19'15" E16022'59”, 870-940m, 24.X.1994, leg. P. Chvojka (9 males, 7 females; NMPC). Germany, Bayern, Böhmerwald, inlet of Kleiner Arbersee, N49 07'24" E13 07'14", 925m, 10.X.2007, leg. P. Chvojka (1 male, 1 female; NMPC). Bayern, Böhmerwald, inlet of Rachelsee, N48 58'34" E1324'03”, 925m, 9.X.2007, leg. P. Chvojka (4 males, 2 females; OPC). Poland, Kudlon Mt. Gorce Mts. 11.XI.1996, leg. B. Szczęsny (4 males, OPC). Gorce Mts. IX-X. leg. B. Szczęsny (3 males, 3 females; OPC). Babia Gora, West Beskidy Mts. 1964, leg. B. Szczęsny (6 males, 5 females; CSNMB). Babia Gora, West Beskidy Mts. 1997, leg. B. Szczęsny (2 males, 2 females; OPC). Dolina Pięciu Stawów Polskich (Lengyel-Öt-tó völgy) Roztoki Spring, the 

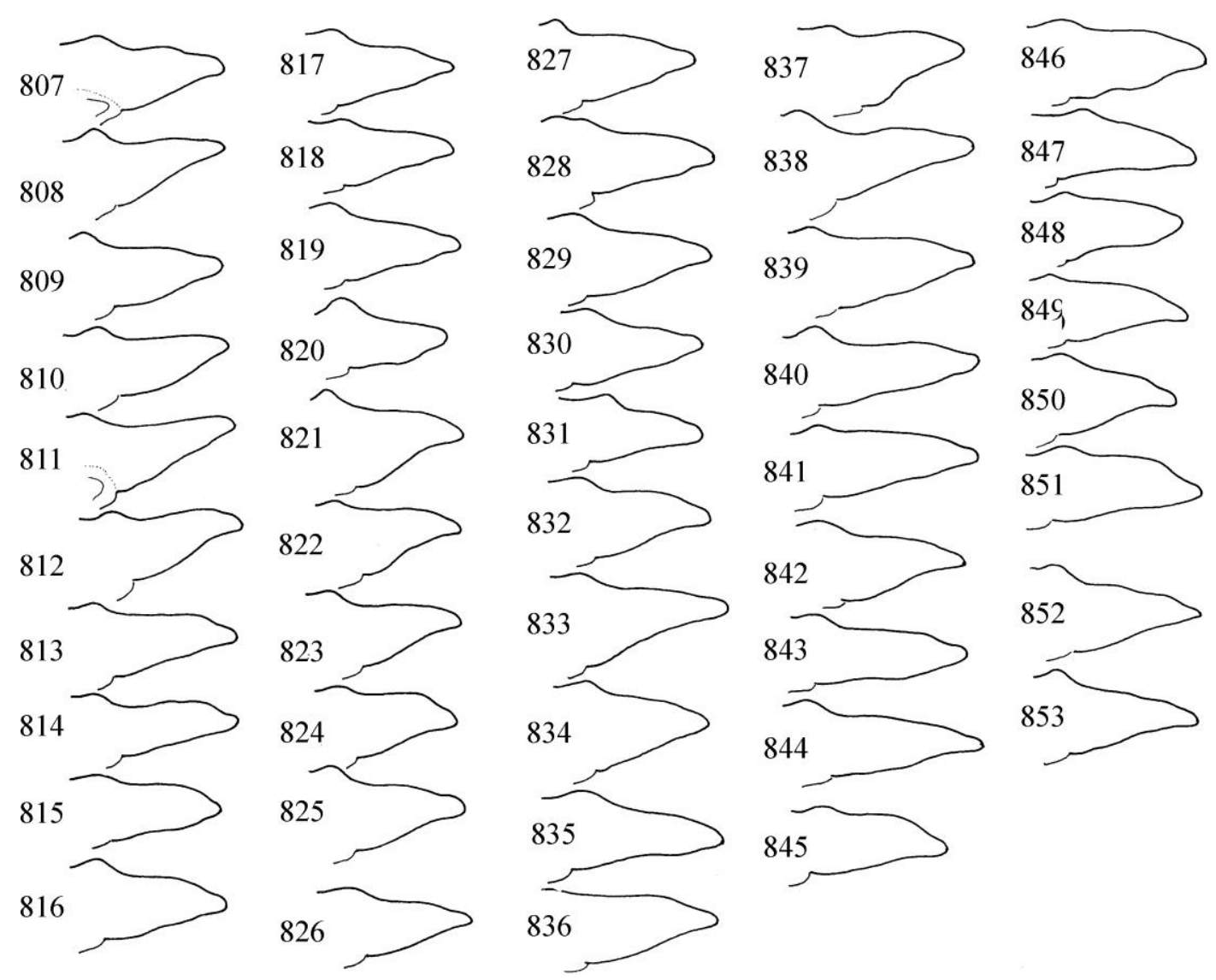

Figures 807-853. Psilopteryx bohemosaxonica Mey \& Botosaneanu, 1985. 807-808 = Austria, Schwarzenberg, 809-812= Germany, Böhmerwald, 813-820 = Czech Republic, Orlické hory Mts., 820-825 = Czech Republic, Sumava Mts., 826-835 = Czech Republic, Jizerské hory Mts., 836-838 = Poland, Gorce Mts., 839-844 = Poland,

Babia Gora Mts., 845-851 = Poland, High Tatra Mts. Rostoka; 852-853 = Slovakia, Poliana Mts.

Tatra Mts. 26.X.1985, leg. B. Szczęsny (3 males, 5 females, OPC). Roztoki Valley, the Tatra Mts. 26.X.1985, leg. B. Szczęsny (5 males, 3 females, OPC). Slovakia, Banskobystrický region, Pol'ana Mts, Hriňová, Bystré, spring brook of Bystrý Stream, N48³7.671' E19²8.655', 1200m 8.X.2013, singled leg. J. Oláh \& L. Szél (1 male, OPC). Banskobystrický region, Pol'ana Mts, Hriňová, Bystré, spring brook of Bystrý Stream, N48³7.569' E19²9.261', 1025m 8.X.2013, singled leg. J. Oláh \& L. Szél (1 male, 1 female; OPC).

\section{Psilopteryx gutinensis Mey \& Botosaneanu, 1985 stat. nov.}

\section{(Figures 854-865)}

Psilopteryx psorosa gutinensis Mey \& Botosaneanu, 1985: 120.

Diagnosis. This sibling incipient species is characterized by short dorsal branch of paraproct.
The lateral profile of the short and high subtriangular paraproct is rather variably. The curvature of the paramere rod is deep. The dental pattern on the paramere head is characterized by an enlarged single leading tooth. This species is distributed in the Ignis and Gutin Mts.

Material examined. Romania, Maramureş county, Muntii Ignis, Deseşti-Staţiunea Izvoare, open brook on the Valhani Plateau, 1020m, N47²3.015' E23444.547', 7.X.2010, leg. P. Barcánfalvi, D. Murányi \& J. Oláh, (1 males, OPC). Maramureş county, 2010/4, Muntii Ignis, Deseşti-Staţiunea Izvoare, brook in bushy edge on the Valhani Plateau, 930m, N47²44.374' E2343.331', 8.X.2010, leg. P. Barcánfalvi, D. Murányi \& J. Oláh, (1 female, OPC). Maramureş county, Muntii Ignis, Deseşti-Staţiunea Izvoare, open brook on the Valhani Plateau, $1020 \mathrm{~m}, \mathrm{~N} 47^{\circ} 43.015^{\prime}$ E2344.547', 21.X. 2010, leg. Á. Ecsedi, J. Oláh \& I. Szivák, (1 male, 1 female, OPC). Maramureş county, Muntii Ignis, Deseşti-Staţiunea Izvoare, open spring brook at settlement, $920 \mathrm{~m}, \mathrm{~N} 47^{\circ} 45.167^{\prime}$ E2343.013', 22.X.2010 leg. Á. Ecsedi, J. Oláh \& I. Szivák, 


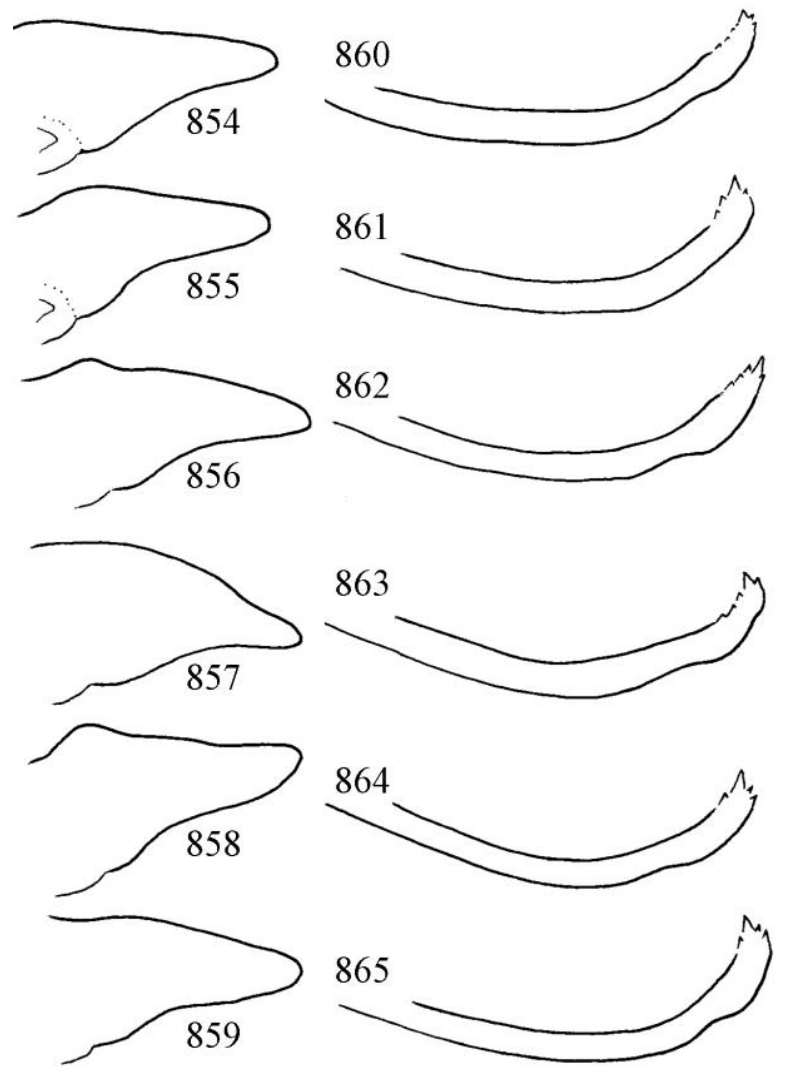

Figures 854-865. Psilopteryx gutinensis Mey \& Botosaneanu, 1985. 949-954 = dorsal branch of paraproct in lateral view, 955-960 $=$ paramere in lateral view.

(3 males, 1 female, OPC). Maramureş county, Muntii Ignis, Deseşti-Staţiunea Izvoare, side valley spring brook along the road between Firiza and Statiunea Izvoare, 600 m, 22.X.2010 leg. Á. Ecsedi, J. Oláh \& I. Szivák, (1male, 1 female, CSNMB).

\section{Psilopteryx javorensis Oláh, sp. nov.}

(Figures 866-875)

Diagnosis. This new sibling incipient species is characterized by short dorsal branch of the paraproct and belongs to the $P$. bohemosaxonica species cluster. Most close to the nominate species $P$. bohemosaxonica, but differs by having the lateral profile of the short and high paraproct very regular triangular at all the examined 5 specimens, not irregular subtriangular. The dental pattern on the paramere head is reduced to a narrowing bifid tip, not densely packed with teeth apicad and dorsosubapicad. This species is distributed in the Javorie (Jávoros) Mts. and in the Pol'ana Mts.
Material examined. Holotype. Slovakia, Javorie Mts, Blyskavica, Tisovnik stream, 657m, 20.X.2005, leg. D. Murányi (1 males, HNHM). Allotype. Same as holotype (1 female, HNHM). Paratypes. Same as holotype (3 males, 1 female HNHM). Banskobystrický region, Pol'ana Mts, Hriňová, sidebrook of Slatina Stream, N48 37.210' E19 31.582', 514m 8.X.2013, singled leg. J. Oláh \& L. Szél (1 male, OPC). Banskobystrický region, Javorie Mts, Stará Huta, Blýskavica, Tisovník Stream, N48 27.553’ E19 18.048', 671m, 7-9.X.2013, singled leg. J. Oláh \& L. Szél (1 female, OPC).

Description. Male (in alcohol). Yellowish brown medium-sized animal with light body appendages and with yellowish-testaceous wings. Anterior wing with rounded apex and with very long erect spine-like setae present on the veins. Tibial spur formula is 033 . Forewing length is 11 $\mathrm{mm}$.

Male genitalia. Segment IX rounded convex in anterior margin in lateral view with very short tergal band and with short rounded dorsal keel. Dorsal branch of paraproct is high and short with very regular triangular shape in lateral view. Cerci with rounded quadrangular lateral lobe, its mesal lobe below the dorsal keel with more pigmented apical region. Gonopods fused to segment IX and represented by a minute just protruding small hump. Lateral wing of the aedeagus well developed. Parameres with shallow curvature and with very simplified dental pattern, just discernible as having a narrowing bifid apex.

Etymology. This new species was named after the mountain range where the type material was collected, Javoros Mts.

\section{Psilopteryx psorosa new sibling species complex}

This sibling incipient species complex is characterized by medium long dorsal branch of paraproct. The length of the dorsal branch of the paraproct is almost identical at the two species. The paraproct is lowest compared to the other species complexes, but little higher at $P$. harmas sp. nov. compared to $P$. psorosa. The dental pattern on the paramere head of $P$. psorosa, the putative ancestral species of the complex, is modified in $P$. harmas sp. nov. The species complex of $P$. psorosa is distributed in both middle and higher elevations of mountain ranges mostly in the western and norther Outer Crapathians. Two species belongs to this complex: P. harmas sp. nov. and $P$. psorosa. 


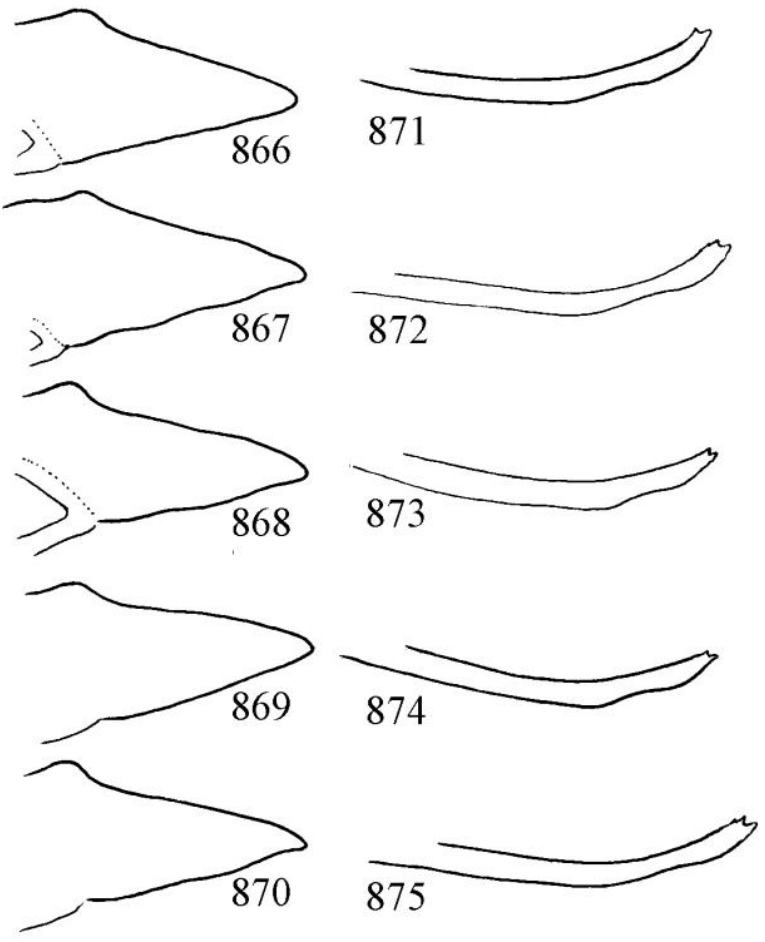

Figures 866-875. Psilopteryx javorensis Oláh \& Chvojka, sp. nov. $866-870=$ dorsal barch of paraproct in lateral view, $871-875=$ paramere in lateral view.

\section{Psilopteryx harmas Oláh \& Chvojka, sp. nov.}

Psilopteryx psorosa carpathica Schmid, 1952. Chvojka 1993: 220. Misidentification.

(Figures 876-889, 890-903)

Diagnosis. This new incipient species is characterized by medium long dorsal branch of the paraproct and therefore belongs to the $P$. psorosa species cluster. Close to the nominate species $P$. psorosa, but differs by having the lateral profile of the paraproct higher, not as low; paramere curvature deeper and the dentate apex turned mesad or upward. It is difficult to demonstrate how its incipient specific status is related to the putative $P$. bohemosaxonicalP. psorosa hybrid cline effect. However, its paraproct and paramere clearly differ from the typical paraproct and paramere structures of pure $P$. psorosa populations. This species might represent a hybrid species of dubious origin and needs further taxonomic and population genetic studies. The formation of new hybrid taxa is possible by introgression of those loci that promote adaptive divergence or reproductive barrier building (Abbott et al. 2013). This species is distributed from the Vihorlat Mts. to the Bieszczady Mts.

Material examined. Holotype. Poland, East Carpathians, Bieszczady Mts. at Wolosatka brook, 900m, 28.X.2010, leg B. Szczęsny (1 male, OPC). Allotype. Same as holotype (1 female, OPC). Paratypes. East Carpathians, Bieszczady Mts. at Wolosatka brook, 850-1000m, 22.X.2014, leg B. Szczęsny (1 male, OPC). East Carpathians, Bieszczady Mts. no more data, leg B. Szczesny (2 males, 1 female; OPC). Slovakia, NE Slovakia, Bukovské Hills, Stuzica stream, left tributary Kremenny potok stream, N49 04'23” E22 ${ }^{\circ} 32^{\prime} 33$ ', 7. XI. 1999, leg J. Lukas (1 male, 2 females; OPC, ). NE Slovakia, Bukovské Hills, Kremenny potok stream, N49 04 '23" E22 32 '33", 8. XI. 1999, leg J. Lukas (1 male, OPC; 1 male, NMPC). East Slovakia, Vihorlat Mts. Cerny potok (above Zemplinske Hamre), 640m, 12.X.1990, leg P. Chvojka (4 males, 1 female, NMPC; 1 male, 1 female; OPC). East Slovakia, Vihorlat Mts. Zemplinske Hamre, stream below source, 700m, 15.X.1962, leg. J. Sykora (5 males, 4 females, NMPC; 2 males, 1 females,OPC; 1 male, 1 female; CSNMB). East Slovakia, Vihorlat Mts. Strihovsky potok brook NW Strihovce, 590-600m, 12.X.1990, leg P. Chvojka (1 male, 3 fgemales; NMPC). East Slovakia, Vihorlat Mts. Malá Bystra, 560-700m, 9.X.1990, leg P. Chvojka (2 males, OPC).

Description. Male (in alcohol). Yellowish brown medium-sized animal with light body appendages and with yellowish-testaceous wings. Anterior wing with rounded apex and with very long erect spine-like setae present on the veins. Tibial spur formula is 033 . Forewing length is 12 mm.

Male genitalia. Segment IX rounded convex in anterior margin in lateral view with very short tergal band and with short rounded dorsal keel. Dorsal branch of paraproct is low and medium long with triangular shape in lateral view. Cerci with rounded and elongated quadrangular lateral lobe, its mesal lobe below the dorsal keel with more pigmented apical region. Gonopods fused to segment IX and represented by a short digitate process. Lateral wing of the aedeagus well developed. Parameres with deep curvature and with pronounced apical dental pattern. Dental apices slightly or variously mesad turning in populations from Beszczady Mts. and from North-East Slovakia. Dental apices variously upward turning in populations from the Vihorlat Mts.

Etymology. harmas from "hármas" triple in Hungarian, refers to the distributional area of the species centred around the triple borders of three countries: Poland, Slovakia and Ukraine. 


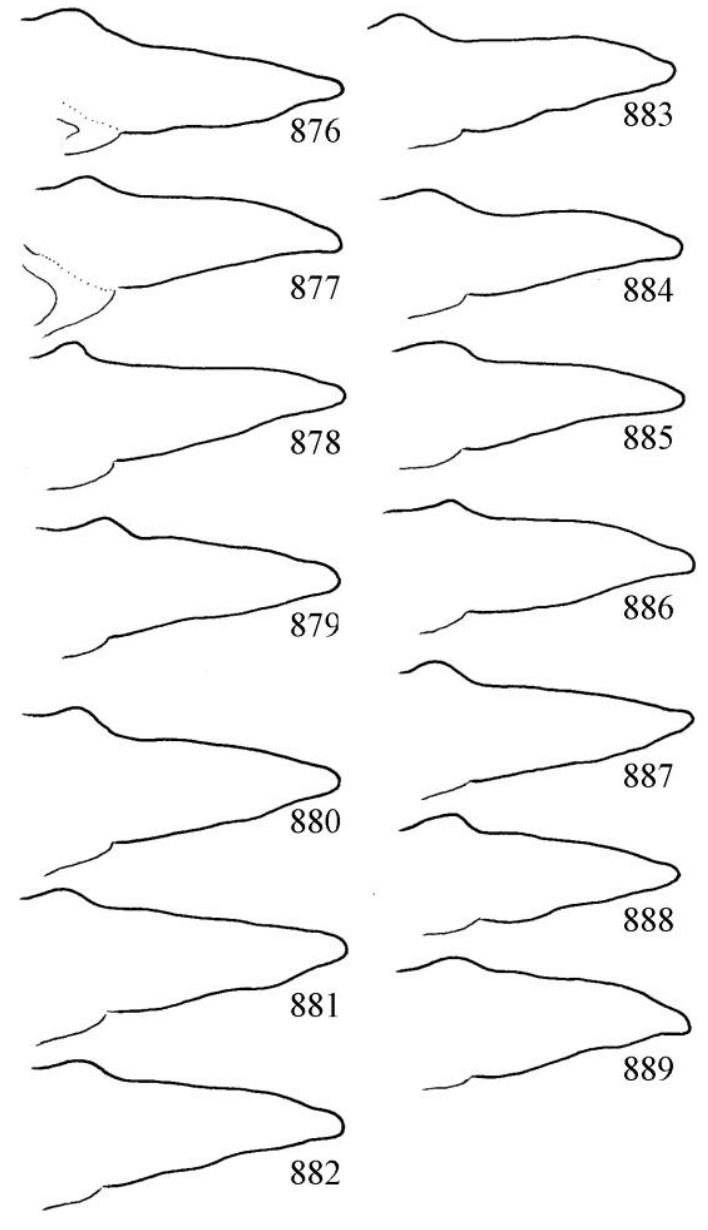

Figures 876-889. Psilopteryx harmas Oláh \& Chvojka, sp. nov. Dorsal branch of paraproct in lateral view. 876-879 = Poland, Bestczady Mts., $880-882=$ North East Slovakia, $883-889=$ Slovakia, Vihorlat Mts.

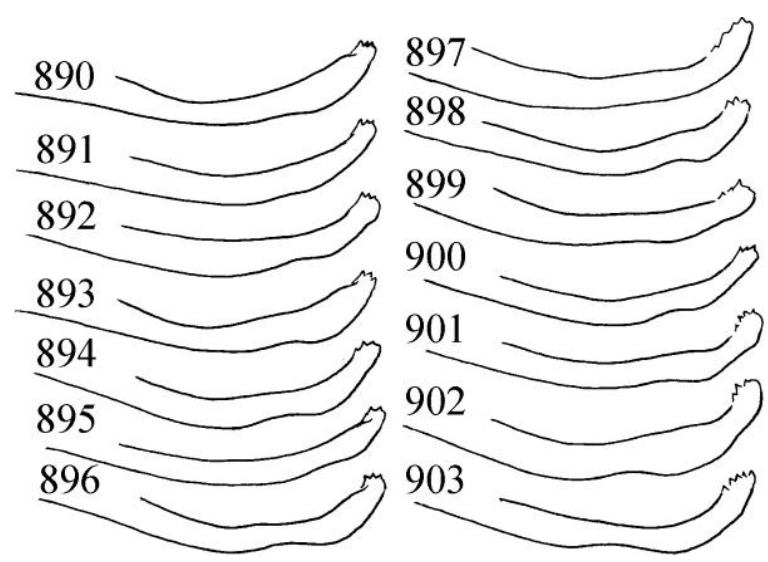

Figures 890-903. Psilopteryx harmas Oláh \& Chvojka, sp. nov. Parameres in lateral view. 890-893 = Poland, Bieszczady Mts., 894-896 = North East Slovakia, 897-903= Slovakia, Vihorlat Mts.

\section{Psilopteryx psorosa (Kolenati, 1860)}

(Figures 904-929, 930-955)

Chaetopteryx psorosa Kolenati, 1860: 388-389.

Psilopreyx psorosa (Kolenati): Psilopteryx genus erected by Stein, 1874: 250. Type species: Chaetopteryx sporosa Kolenati (monotypic).

Psilopteryx psorosa carpathica Schmid, 1952: Szczęsny 1986: 537. Misidentification.

Diagnosis. This incipient species is characterrized by medium long dorsal branch of the paraproct. The lateral profile of the paraproct is subtriangular and very low, the lowest compared to all the other species. The curvature of the paramere rod is shallow. The dental pattern on the paramere head is concentrated on the very apical region. Individual variation and intermediate cline formations might be connected with complex contact zones with $P$. bohemosaxonica. This species is distributed in the Hrubý Jeseník Mts, Králicky Snéznik Mt., Sudety, Tatras, Babia Hora Mts. Frequently intermingled with $P$. bohemosaxonica, probably in a complicated cline system. Its distribution and hybrid formation needs further studies as was suggested already long before (Mey \& Botosaneanu 1985).

Material examined. Czech Republic, Jesenik, Ovcarna, source of Bila Opava, 1300m, 24. IX. 2000, leg. I. Sivec \& B. Horvát, near type locality ( 5 males, OPC, 5 males, 1 female; CSNMB). Northern Moravia, Králicky Snéznik Mt., Morava River below Králicky Snéznik Mt., N50'11'59" E16 ${ }^{\circ} 50$ '36”, 1100m, 19.X.2000, leg. P. Chvojka (6 males, 3 females; NMPC). Eastern Bohemia, Králicky Snéznik Mt., brook, "Strasidla" SW Králicky Snéznik Mt., N50¹1'35" E16 49'39", 1070m, 19.X.2000, leg. P. Chvojka (14 males, 5 females; NMPC). Poland, Right tributary of the Olczyski Potok, (Olczyski Spring), the Tatra Mts., 12.XI.1986, leg. B. Szczęsny (16 males, 5 females, OPC). Kościeliska Valley, the Tatra Mts. 25.X.1985, leg. B. Szczęsny (6 males, 5 females, OPC). Tatra Mts., IX-X. leg. B. Szczęsny (2 males, 2 females, OPC). The Biała Lądecka River, Masyw Śnieżnika, The East Sudety, N 50 14' $17^{\prime \prime}$ E $17^{\circ} 0^{\prime} 5.76^{\prime \prime}$, 9.XI.2009, leg. K. Majecka (1 female, OPC). The Kleśnica River, Masyw Śnieżnika, The East Sudety, N N 50 15' 35" E $16^{\circ} 51^{\prime} 41^{\prime \prime}, 10 . X I .2009$, leg. K. Majecka (1 male, OPC). Biały Spław, beginnig of stream the Biała Lądecka River, Masyw Śnieżnika, The East Sudety, N 50 $13^{\prime} 47^{\prime \prime}$ E $17^{\circ} 1^{\prime}$ 4", 9.XI.2009, leg. K. Majecka (1 female, OPC). Karkonoski Park Narodowy (Giant Mts.), Hala Szrenicka, Kamienczyk stream, spring area, 11.X.2014, leg. J. Majecki (1 male, OPC). Karkonoski Park Narodowy (Giant Mts.), Równia pod Sniezka, spring area, 12.X.2014 leg. J. Majecki (1 male, 1 female in copula; OPC). Slovakia, Vysoké Tatry, brooks E of 

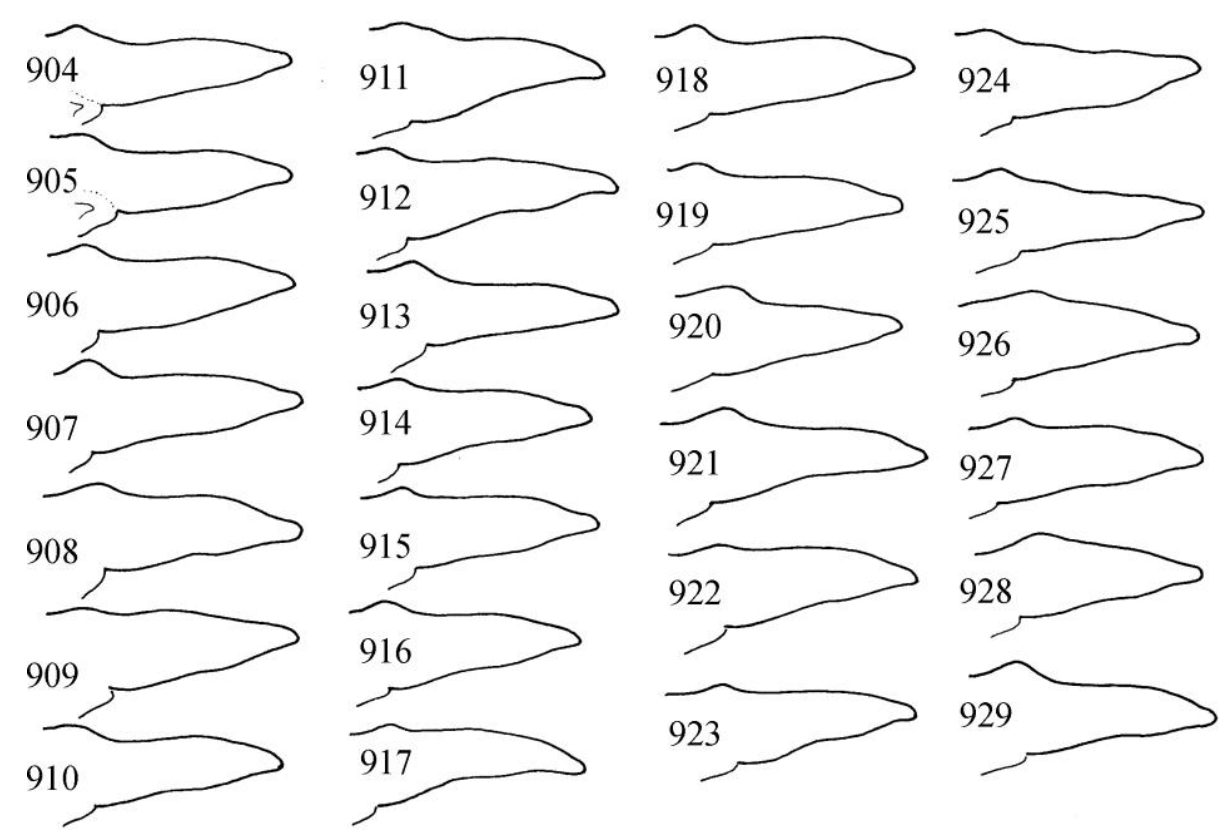

Figures 904-929. Psilopteryx psorosa (Kolenati, 1860). Dorsal barch of paraproct in lateral view. 904-913 = Czech Republic, Jesenik; 914-924 = Czech Republic, Kralicky Snéznik Mt., 925-929 = Poland, High Tatra, Koscielska.
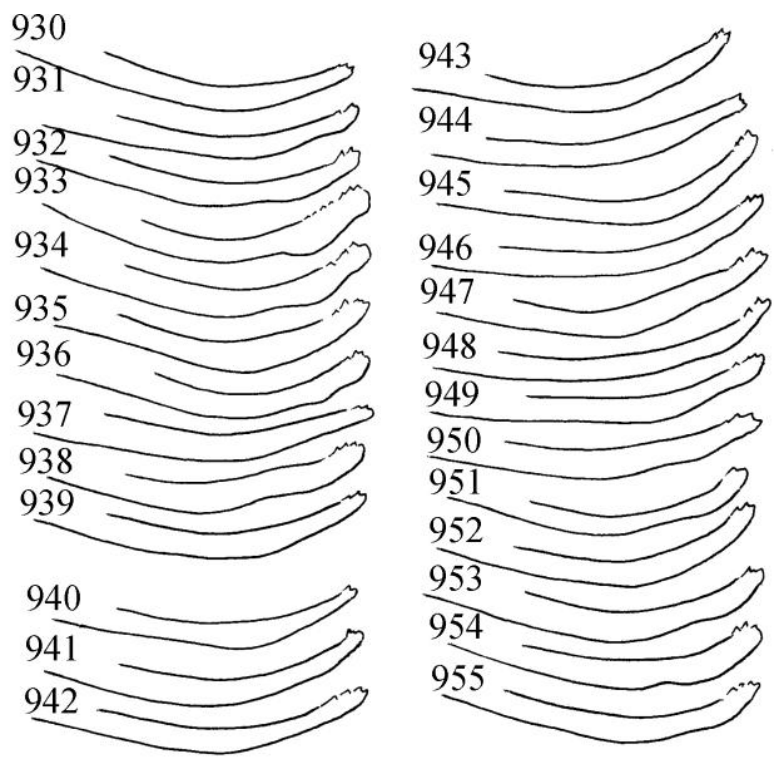

Figures 930-955. Psilopteryx psorosa (Kolenati, 1860) Parameres in lateral view. 930-938 = Czech Republic, Jesenik; 939-949 = Czech Republic, Kralicky Snéznik Mt., 950-955 = Poland, High Tatra, Koscielska.

Zlomiskovy potok brook, N4908'14" E2001'05", 1460m, 7.X.1989, leg. P. Chvojka (3 males, NMPC). Vysoké Tatry, Furkotsky potok brook, N4908'014" E2002'015”, 1480m, 7.X.1989, leg. P. Chvojka (1 female, NMPC). Vysoké Tatry, Zlomiskovy potok brook, N4907'18' E20 00'59”, 1460m, 7.X.1989, leg. P. Chvojka (1 male, 1 female, NMPC). Pod- tatranská kotlina basin, Lieskovec, N49 $07^{\prime} 13^{\prime \prime}$ E20 03 '04”, 1280m, 7.X.1989, leg. P. Chvojka (1 male, 1 female; NMPC). Podtatranská kotlina basin, Nové Strbské pleso, N49 07'04" E2003'56”, 1315m, 7.X.1989, leg. P. Chvojka (2 males, NMPC). Podtatranská kotlina basin, right tributary of Bela stream, SW Hrdovo (NEPribylina) N49 $07^{\prime} 04^{\prime \prime}$ E19 50'45”, 830m, 11.X.1989, leg. P. Chvojka (2 males, 3 females; NMPC). Oravské Beskydy Mts. left tributary of Bystrá stream below Babia hora Mt., N49³3'39" E19 30'47', 1300m, 15.X.1991, leg. P. Chvojka (6 males, 4 females; NMPC).

\section{Psilopteryx carpathica new sibling species complex}

This sibling incipient species complex is characterized by elongated dorsal branch of paraproct. The length of the dorsal branch of the paraproct is further diverging among the three species. The paraproct is longest at $P$. transylvanica, shortest at $P$. carpathica. The dental pattern on the paramere head of $P$. carpathica very simplified; dental pattern most elaborated at $P$. retezatica. The species complex of $P$. carpathica is distributed in the higher elevations of mountain ranges mostly in the eastern and southern Outer Crapathians. Three species belongs to this complex: $P$. carpathica, $P$. transylvanica and $P$. retezatica. 
Psilopteryx carpathica Schmid, 1952 stat. restit.

(Figures 956-969, 970-983)

Psilopteryx carpathica Schmid, 1952: 142-144.

Psilopteryx psorosa carpathica Schmid, 1952: Mey \& Botosaneanu 1985: 120, downgraded to subspecies level.

Diagnosis. This incipient nominate species of the complex is characterized by long dorsal branch of the paraproct. The lateral profile of the paraproct is elongated subtriangular and the lowest compared to the two other species. The curvature of the paramere rod is shallow. The deeper curvature found at the single specimen examined from the Gorgany Mts. could be a result of copulatory processes. The simplified dental pattern on the paramere head is restricted to the very apical almost bifid tip. This species populates spring fed streams in the Gorgany, Czarnohora, Maramaros, and Rodnai Mts. of the North-East Carpathians.

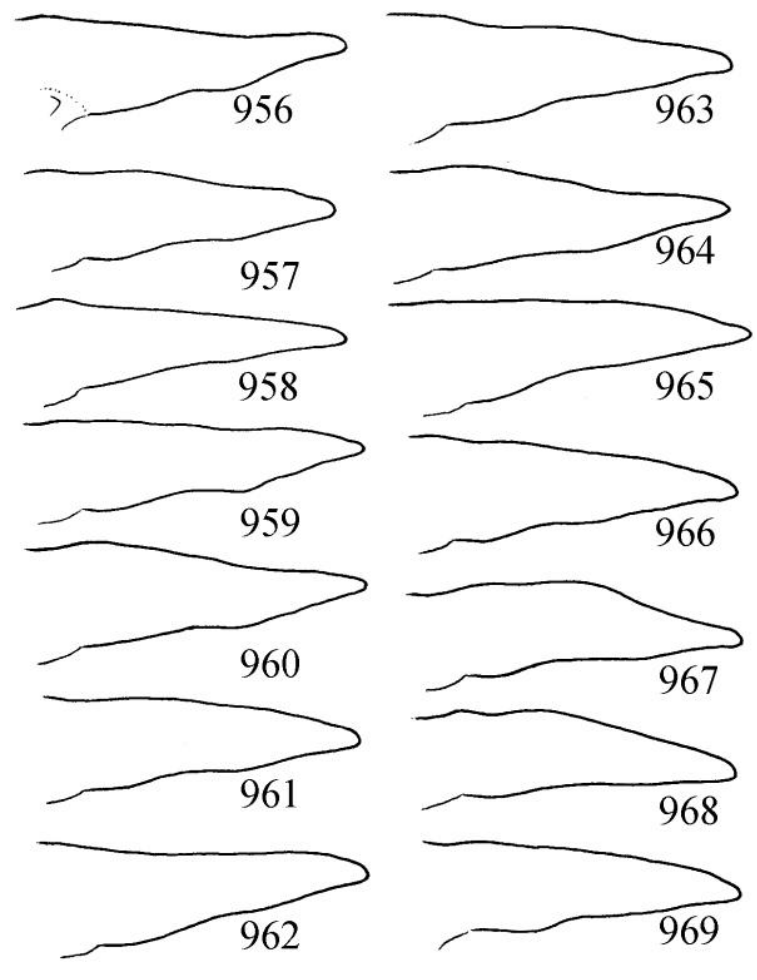

Figures 956-969. Psilopteryx carpathica Schmid, 1952. Dorsal branch of paraproct in lateral view. 956-960= Ukraine, Czarnohora Mts., 961-964 = Ukraine, Skupova Mts., 965 = Ukraine, Gorgany Mts., 966-969 = Romania, Rodna Mts.
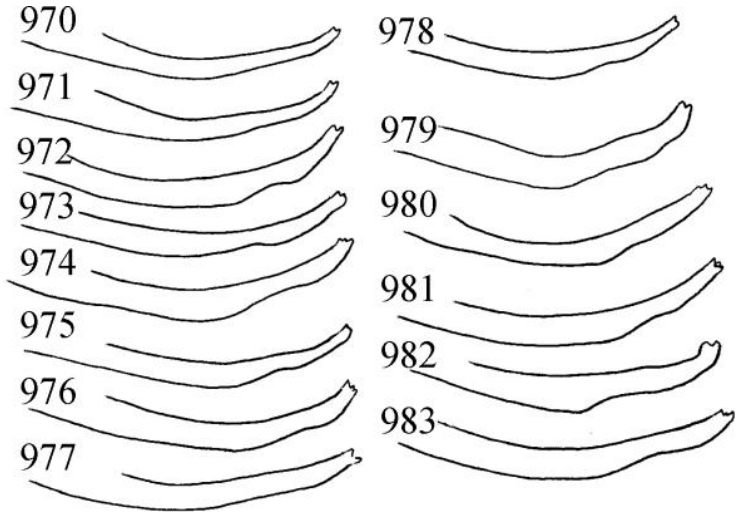

Figures 970-983. Psilopteryx carpathica Schmid, 1952. Paramere in lateral view. 970-974 = Ukraine, Czarnohora Mts., 975-978 = Ukraine, Skupova Mts., $979=$ Ukraine, Gorgany Mts., 980-1983 = Romania, Rodna Mts.

Material examined. Romania, Borsa, 26.IX.1992, leg J. Oláh, (1 male, OPC). Maramures county, Rodna Mts. BorsaStatiunea Borsa, stream along the road towards Prislop Pass, 1014 m, N47 $37^{\prime} 34.0^{\prime \prime}$ E24 49' 13.0'”, 26.IX.2006, leg. L. Dányi, J. Kontschan \& D. Murányi (1 male, HNHM). Maramures county, Maramures Mts. Borsa-Baile Borsa, brook over the village $1046 \mathrm{~m}, \mathrm{~N} 47^{\circ} 40^{\prime} 21.5^{\prime}$ ' E24 ${ }^{\circ}$ 50' 16.7', 26.IX.2006 leg. L. Dányi, J. Kontschán \& D. Murányi (1 male deformed, HNHM). Radnei Mts. numerous spring streamlets on the spring area of Cailor waterfall, Piatra Rea, N47³5'1.9” E2447'49.4”, 1564m, 28. IX. 2014, leg. J. Oláh $\&$ Cs. Balogh (1 male, OPC). Radnei Mts. small spring below Lake Isvoru Bistritei, N47 34'46.4” E244' 49.34", 1586m, 28. IX. 2014, leg. J. Oláh \& Cs. Balogh (1 male, 1 female; OPC). Ukraine, East Carpathians, Gorgany Mts., at Sitny brook, 1111m, 29.X.2005, leg B. Szczęsny (1 male, 1 female; OPC). East Carpathians, Skupova Mts., Hnylec brook, 1470 m, 26.X.2006, leg B. Szczęsny (2 males, 2 females; OPC, 2 males, 1 female; CSNMB). Czarnohora Massif, Prut River at Foreszczynka, 26.X.2005, leg. B. Szczęsny (1 male, 1 female; OPC). Czarnohora Massif, 8.X.19955, leg. B. Szczęsny (4 males, 1 female; OPC).

\section{Psilopteryx retezatica Botosaneanu \& Schneider, 1978 stat. nov.}

(Figures 984-1008, 1009-1033)

Psilopteryx psorosa retezatica Botosaneanu \& Schneider, 1978: 321-322.

Psilopteryx psorosa retezatica Botosaneanu \& Schneider, 1978: Mey \& Botosaneanu 1985: 119.

Diagnosis. This incipient species of the complex is characterized by long dorsal branch of the paraproct. The lateral profile of the paraproct is irregular subtriangular and the highest in the spe- 
cies group. The curvature of the paramere rod is deeper compared to the other two species of the complex and having constrictions and dilatations more frequently. The dental pattern on the paramere head is most elaborated in the entire species group. Dentate pattern moves more far into the middle direction in lateral view. This species populate spring fed streams in the Tarcu, Retezat, Muntele Mic, Cerna, and Parang Mts. In Parang Mts. there are sign of hybrid contact zone with $P$. transsylvanica in the shape or dental pattern of the speciation traits of paraproct and paramere.

Material examined. Romania, Retezat Mts., Gura Apelor, N45.33 E22.88, 1500m, 20.X.2007, leg. M. Bálint, E. Magyari \& M. Braun (23 males, 13 females; OPC). Retezat Mts. $24 \mathrm{~km}$ from Baile Herculane, spring area of a small tributary to River Cerna, N45 ${ }^{\circ} 2^{\prime} 32.14^{\prime \prime}$ E22 ${ }^{\circ} 35^{\prime} 3.36 "$, 13.XI.2010, singled leg. Á Ecsedi \& I. Szivák (2 males, OPC). Caraş-Severin county, Țarcu Mts. left side brook of open stream on the $\mathrm{N}$ slope of Mt. TTarcu, $1500 \mathrm{~m}$, N4517'40.7”, E22³1'44.5”, 14.10.2011, leg. Á. Ecsedi, T. Kovács, G. Puskás, (1§, OPC). Caraş-Severin county, Ţarcu Mts. spring and its outlet at Cuntu Meteorological Station, N45 $18^{\prime} 00.2^{\prime \prime}, \mathrm{E} 22^{\circ} 30^{\prime} 04.3^{\prime \prime}, 1465 \mathrm{~m}, 14.10 .2011$, leg. Á. Ecsedi, T. Kovács, G. Puskás, (12 males,5 females, OPC; 7 males, 3 females; CSNMB). Vâlcea county Parâng Mts, Obrâşia Lotrului, open brook, $200 \mathrm{~m}$ of Transalpina (67C) road, 45'22’46.1”, 2338’30.6”, $1765 \mathrm{~m}$, 9.XI.2014, leg. T. Kovács \& G. Magos ( 3 males, 3 females, OPC). Vâlcea county, Parâng Mts, Obrâşia Lotrului, open brook, $900 \mathrm{~m}$ of Transalpina (67C) road, 45²3'9.9', 2339'24.9', $1780 \mathrm{~m}$, 9.XI.2014, leg. T. Kovács \& G. Magos (2 males, 1 female, OPC). Vâlcea county, Parâng Mts, Obrâşia Lotrului, open spring area, $100 \mathrm{~m}$ of Transalpina (67C) road, 45 $22^{\prime} 27.7^{\prime \prime}$, 2339’4.0”, 1915 m, 9.XI.2014, leg. T. Kovács \& G. Magos (1 male, OPC).

\section{Psilopteryx transylvanica Mey \& Botosaneanu, 1985 stat. nov.}

(Figures 1034-1054, 1055-1075)

Psilopteryx (Psilopteryx) carpathica Schmid, 1952: Botosaneanu 1957: 98-99. Misidentification.

Psilopteryx psorosa transylvanica Mey \& Botosaneanu, 1985: 120.

Diagnosis. This incipient species of the complex has long dorsal branch of the paraproct. The lateral profile of the paraproct is characterized by almost regular straight dorsum and downward directed basal region. The curvature of the paramere
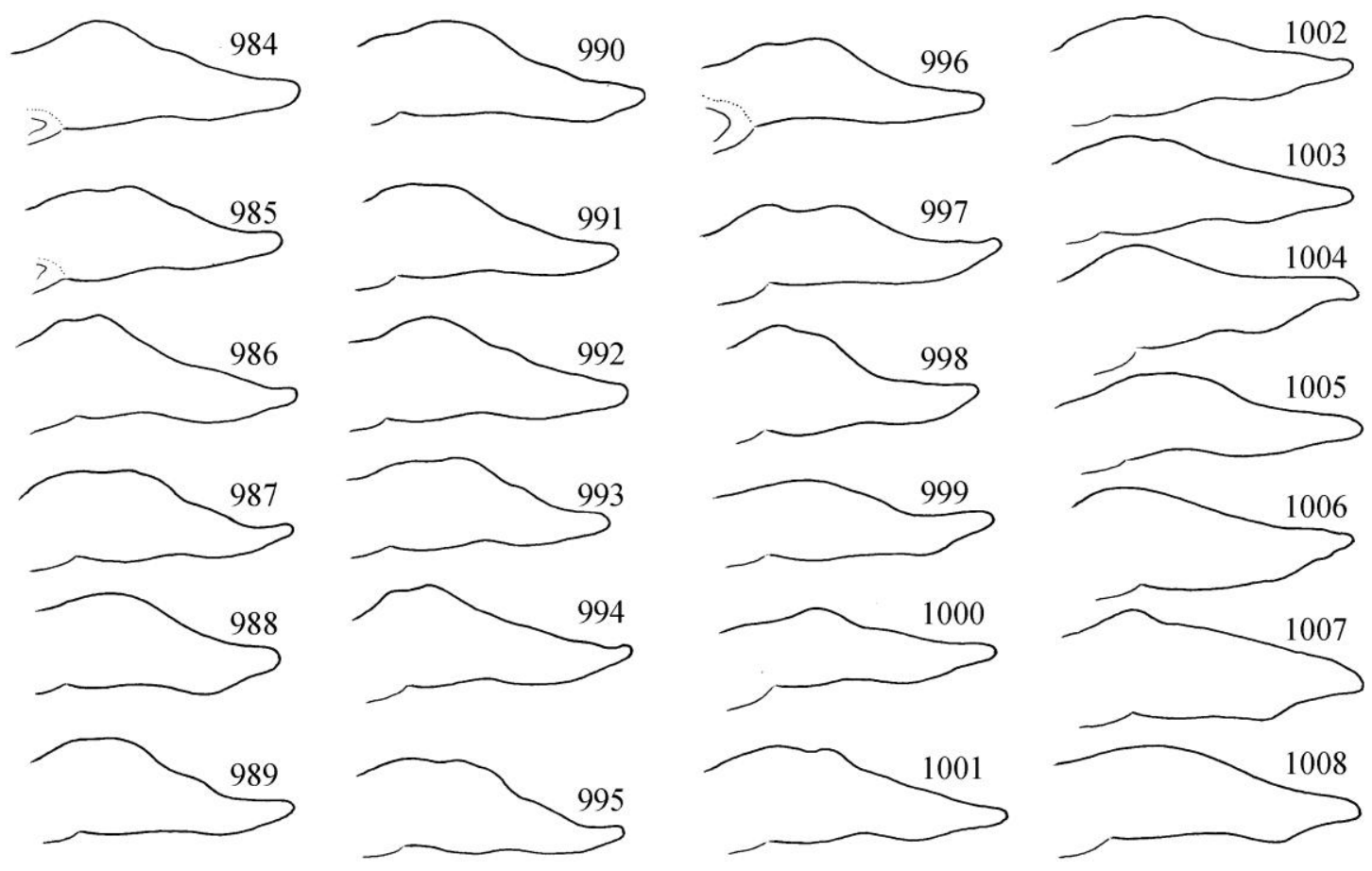

Figures 984-1008. Psilopteryx retezatica Mey \& Botosaneanu, 1985. Dorsal barch of paraproct in lateral view. 984-996 = Romania, Tarcu Mts., 997-1003 = Romania Retezat Mts. 1004-1008 = Romania, Parang Mts. 


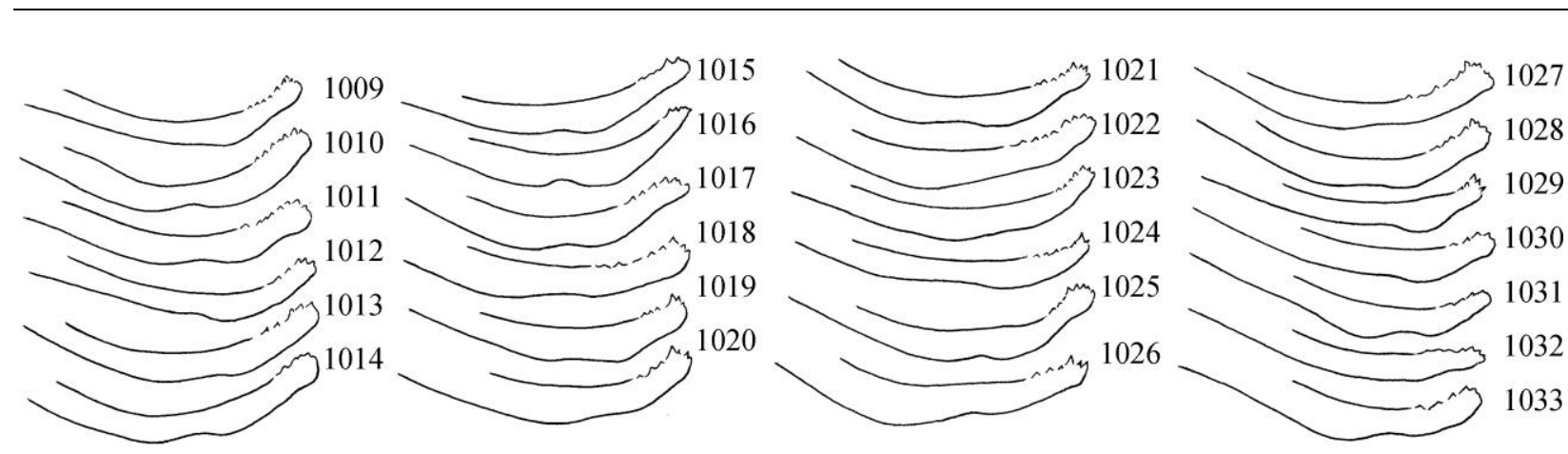

Figures 1009-1033. Psilopteryx retezatica Mey \& Botosaneanu, 1985. Paramere in lateral view. 1009-1021 = Romania, Tarcu Mts., 1022-1028 = Romania Retezat Mts. 1029-1033 = Romania, Parang Mts.
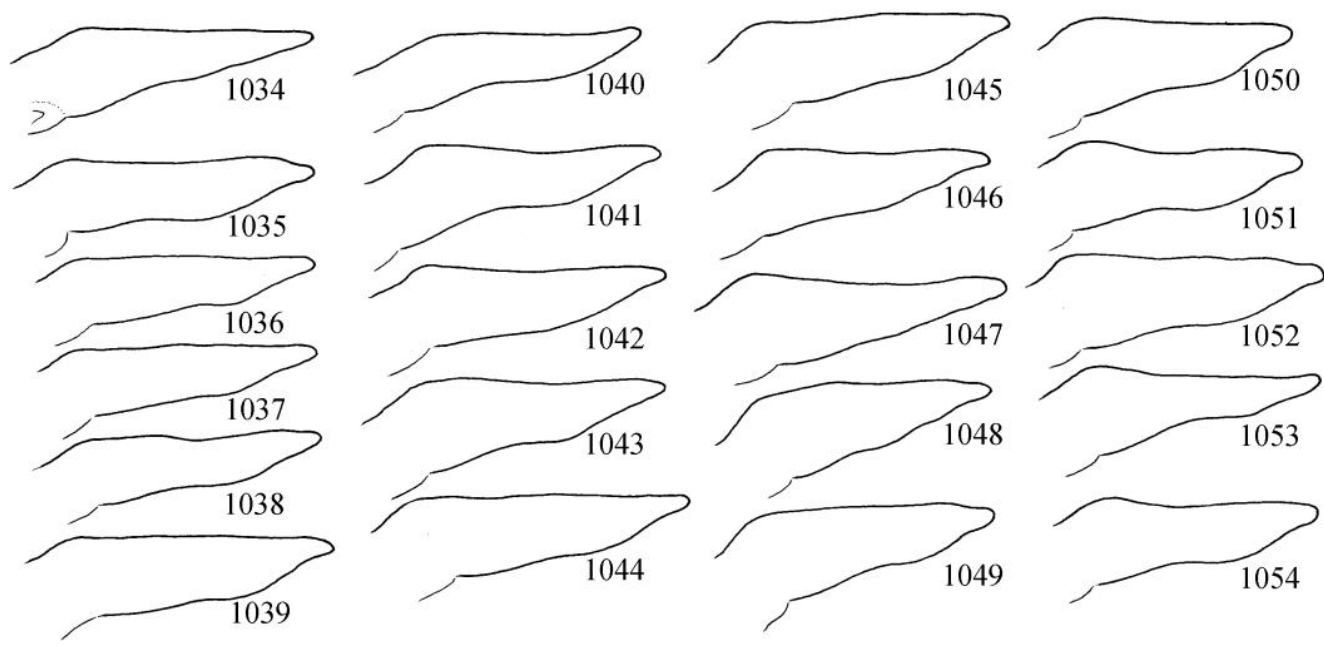

Figures 1034-1054. Psilopteryx transylvanica Mey \& Botosaneanu, 1985. Dorsal barch of paraproct in lateral view. 1034-1036 = Romania, Gurghiu Mts., 1037-1040 = Romania, Hargitha Mts., 1041-1043 = Romania, Bucegi Mts. 1954; 1044-1047 = Romania, Bucegi Mts. 2014; 1048-1054 = Romania, Fagaras Mts.

rod is shallow and having constrictions and dilatations more frequently in the most western populations in the Fagaras Mts. The dental pattern on the paramere head is restricted to apicad and dorsosubapicad. Dentate pattern does not move more far into the middle direction in lateral view, except again in populations of the Fagaras Mts. The contact zone with $P$. retezatica could be more complicated extending even into the Fagaras Mts. This species is most distributed in the complex; populating spring-fed small streams on higher elevations in mountan ranges in the eastern and southern Carpathians. We have collected numerous specimens in Gurghiu, Hargitha, Bucegi and Fagaras Mts.

Material examined. Romania. Parcul Natural Bucegi, Peles, 7.X.1954, leg. P. Iuncu (1 male, OPC). Parcul Natural
Bucegi, V. Pelesului, 4.X.1954, leg. P. Iuncu (4 males, OPC). Parcul Natural Bucegi, Peles, 27.X.1954, leg. P. Iuncu (2 males, 1 female; OPC). Parcul Natural Bucegi, Parcul Natural Cascada Urlatoarea, 22.X.1954, leg. P. Iuncu (2 males, 1 female; OPC). Gurghiu Mts. near Bucin Pass, Tárnava Mica springs and stream, N: 46 $39^{\prime} 16,63$ 'E: $25^{\circ} 16^{\prime} 42,46^{\prime}$ ', 1290 , 30.X.2014, leg. Z. Baczó, Cs. Balogh, J. Kecskés \& J. Oláh. (13 males, 9 females; OPC). Gurghiu Mts. near Bucin Pass, Gainasa springs and stream, N: 46 $40^{\prime} 11,35^{\prime}$ E: $25^{\circ} 17^{\prime}$ 39,06”, 1400, 30.X.2014, leg. Z. Baczó, Cs. Balogh, J. Kecskés \& J. Oláh (66 males, 7 females; OPC). Gurghiu Mts. near Bucin Pass, Frasileasa stream with side springs, N46 $38^{\circ}$ 37,45” E: 2517’ 35,08”, 1193, 29.X.2014, leg. Z. Baczó, Cs. Balogh, J. Kecskés \& J. Oláh. ( 8 males, 3 females; OPC). Hargitha Mts. Filio stream side spring, N: $46^{\circ} 27^{\prime} 03,90^{\prime \prime} \mathrm{E}$ : 2533' 29,29”, 1350m, 31.X.2014 leg. Z. Baczó, Cs. Balogh, J. Kecskés \& J. Oláh. (9 males, 5 females; OPC). Hargitha Mts. Filio stream side spring, N: $46^{\circ} 27^{\prime} 14,53^{\prime \prime}$ E: $25^{\circ} 33^{\prime}$ 53,04", 1415m, 31.X.2014 leg. Z. Baczó, Cs. Balogh, J. Kecskés \& J. Oláh. ( 2 males, 2 females; OPC; 10 males, 4 females; CSNMB). Hargitha Mts. Filio stream side spring, N 


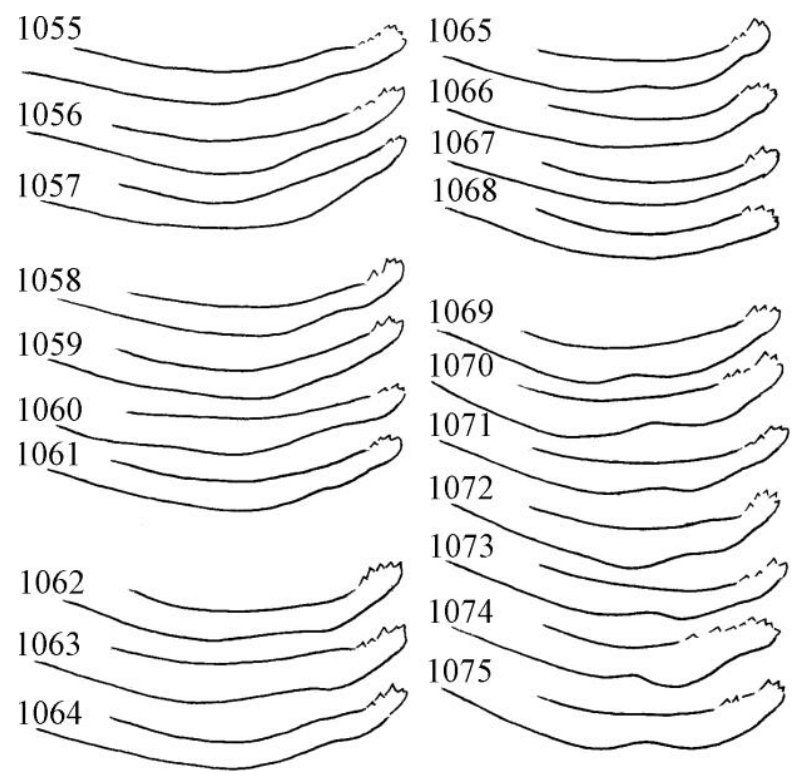

Figures 1055-1075. Psilopteryx transylvanica Mey \& Botosaneanu, 1985. Paramere in lateral view. 1149-1151 = Romania, Gurghiu Mts., 1152-1155 = Romania, Hargitha Mts., 1156-1158 = Romania, Bucegi Mts. 1954; 1159-1162 = Romania, Bucegi Mts. 2014; 1163-1169 = Romania, Fagaras Mts.

46²6’ 45,18” E: 2534' 25,73”, 1600m, 31.X.2014 leg. Z. Baczó, Cs. Balogh, J. Kecskés \& J. Oláh. (7 males, OPC). Hargitha Mts. Filio stream side spring, N: $46^{\circ} 26^{\prime} 29,54^{\prime \prime}$ E: 2534' 48,09”, 1625m, 31.X.2014 leg. Z. Baczó, Cs. Balogh, J. Kecskés \& J. Oláh. (29 males, 9 females, OPC). Dâmbovia county, Bucegi Mts, M. Dichiu, left sidebrook of V.

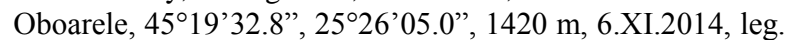
T. Kovács \& G. Magos (2 males, 1 female; OPC). Dâmbovia county, Bucegi Mts, Hotel Peştera, Valea Cocora, 45 $23^{\prime}$ 04.1”, 25²6’37.6”, 1590 m, 6.XI.2014., leg. T. Kovács \& G. Magos (1 male, 1 female; OPC). Dâmbovia county, Bucegi Mts, Hotel Peştera, V. Şugărilor, 4524'42.1”, 2527'23.5”, 1850 m, 6.XI.2014, leg. T. Kovács \& G. Magos (1 male, OPC). Dâmbovia county, Bucegi Mts, Hotel Peştera, left

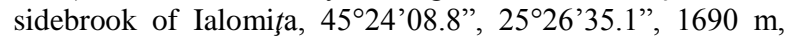
6.XI.2014, leg. T. Kovács \& G. Magos ( 9 males, 7 females, OPC). Dâmbovia county, Bucegi Mts, Hotel Peştera, spring area beside the V. Şugărilor, $45^{\circ} 24^{\prime} 25.0^{\prime \prime}, 25^{\circ} 26^{\prime} 47.8^{\prime \prime}, 1760$ m, 6.XI.2014, leg. T. Kovács \& G. Magos (5 males, 3 females, OPC). Dâmbovia county, Bucegi Mts, Hotel Peştera, Ialomiţa, 45²3'54.5”, 25²6’25.1”, 1610 m, 7.XI.2014 leg. T. Kovács \& G. Magos (1 male, 1 female, OPC). Dâmbovia county, Bucegi Mts, Hotel Peştera, spring area beside the Cascada Obrisia Ialomiţei, 452 $5^{\prime} 35.2,25^{\circ} 26^{\prime} 42.8^{\prime \prime}, 2030 \mathrm{~m}$, 7.XI.2014, leg. T. Kovács \& G. Magos (3 males, 1 female, OPC). Dâmbovia, Bucegi Mts, Lacul Bolboci, Blana Stream, 45²2'06.0”, 2526'40.9”, 1515 m, 7.XI.2014, leg. T. Kovács \& G. Magos ( 2 males, 3 females, OPC). Sibiu county, Făgăraş Mts, Cârţiş̧oara, spring beside the Bâlea Stream, 45³7'59.4”, 2436’31.3”, 1290 m, 8.XI.2014, leg. T. Ko- vács \& G. Magos (13 males, 11 females, OPC). Sibiu county, Făgăraș Mts, Cârţișoara, open sidebrook of Bâlea Stream below the Bâlea Lake, 45³6'30.4”, 24³7'14.6”, 1940 m, 8.XI.2014, leg. T. Kovács \& G. Magos (1 male, 2 females, OPC). Sibiu county, Făgăraș Mts, Cârțișoara, Bâlea Stream below the Bâlea Lake, 45³6'30.4”, 2437'14.6”, 1940 m, 08.11.2014, leg. T. Kovács \& G. Magos (1 male, 2 females, OPC).

Acknowledgements - We are deeply thankful to Hans Malicky for initiating this study and we thank his generous help in making available for us his valuable material. Important materials have been provided and our studies supported by Benjamin Price, The Natural History Musem, London, England; by Dmitri Logunov, The Manchester $\mathrm{Mu}$ seum, University of Manchester, England; by Ian Wallace, National Museums Liverpool, England; by Marcos A. González, University of Santiago de Compostela, Spain; by Carmen Zamora-Muñoz, Universidad de Granada, Spain; by Constantin Ciubuc, Sinaia Zoological Research Station, University of Bucharest, Romania; by Stoyan Beshkov, National Museum of Natural History, Sofia, Bulgaria. We thank Wolfram Graf, Mladen Kucinic, Ana Previsic, Simon Vitecek for their primary and comparative materials sent to us. We are also thankful to Miklós Bálint, Tibor Kovács, Dávid Murányi, Ildikó Szivák, Ákos Uherkovics for their materials as well as for their valuable helps during our field studies and in our collecting efforts. We are grateful for their ideas, information and advices giving to us during the entire five years period of this research.

\section{REFERENCES}

Aвотт, R et al. (with 38 co-authors) (2013): Hybridization and speciation. Journal of Evolutionary Biology, 26:229-246. doi: 10.1111/j.1420-9101.2012.02599.x

AdAMs, D.C., RohlF, F.J. \& SliCE, D.E. (2013): A field comes of age: geometric morphometrics in the $21^{\text {st }}$ century. Hystrix, the Italian Journal of Mammalogy, 24(1):7-14. doi: $10.4404 /$ hystrix-24.1-6283

AGosti, D. (2003): Encyclopedia of life: should species description equal gene sequence? Trends in Ecology and Evolution, 18(6): 273. doi:10.1016/S0169-5347(03)00099-5

Ah-King, M., BArron, A B. \& Herberstein, M.E. (2014): Genital evolution: why are females still understudied? PLOS Biology, 12(5). doi: 10.1371/journal.pbio.1001851

Albert, F.W. \& KRUglyaK, L. (2015): The role of regulatory variation in complex traits and disease. Nature Reviews Genetics, 16:197-212. doi: $10.1038 / \operatorname{nrg} 3891$

Anderson, J.T., WAgner, M. R., Rushworth, C.A., PRASAD, K.V.S.K. \& OLDS, T.M. (2014): The evo- 
lution of quantitative traits in complex environments. Heredity, 112:4-12.

doi: $10.1038 /$ hdy. 2013.33

ARNQVIST, G. (1997): The evolution of animal genitalia: distinguishing between hypotheses by single species studies. Biological Journal of the Linnaean Society, 60: 365-379. doi:10.1006/bijl.1996.0109

ARNQVIST, G. (1998): Comparative evidence for the evolution of genitalia by sexual selection. Nature, 393: 784-786. doi: $\underline{10.1038 / 31689}$

BALLARD, J.W.O. \& WHITLOCK, M.C. (2004): The incomplete natural history of mitochondria. Molecular Ecology, 13:729-744 doi: 10.1046/j.1365-294X.2003.02063.x

BALLARD, J.W.O. \& RAND, D.M. (2005): The population biology of mitochondrial DNA and its phylogenetic implications. Annual Rewiev of Ecology, Evolution and Systematics, 36: 621-642. doi: 10.1146/annurev.ecolsys.36.091704.175513

BICKFORD D., LOHMAN, D.J., SODHI, N.S., NG, P.K.L., MEIER, R., Winker, K., INGRAM, K.K. \& DAS, I. (2006): Cryptic species as a window on diversity and conservation. Trends in Ecology and Evolution, 22(3): 148-155. doi: 10.1016/j.tree.2006.11.004

Bierne, N., Welch, J., Loire, E., Bonhomme, F. \& DAVID, P. (2011): The coupling hypothesis: why genome scans fail to map local adaptation genes. Molecular Ecology, 20: 2044-2072. doi: 10.1111/j.1365-294X.2011.05080.x

BIRKY, C.W. (2001): The inheritance of genes in mitochondria and chloroplasts: laws, mechanisms, and models. Annual Review of Genetics, 35: 125-148. doi: 10.1146/annurev.genet.35.102401.090231

BONDURIANSKY, R. (2011): Sexual selection and conflict as engines of ecological diversification. The American Naturalist, 178(6): 729-745. doi: $\underline{10.1086 / 662665}$

BONDURIANSKY, R. (2012): Rethinking heredity, again. Trends in Ecology and Evolution, 27(6): 330336. doi: 10.1016/j.tree.2012.02.003

Botosaneanu, L. (1947): Rechersces sur les Trichopteres (imagos) de Roumania. Bulletin Entomologique de la Pologne, 26(25): 383-433 + Pls. IXXVI.

BotosAnEANU, L. (1952): Carpathopsyche lateroproducta n. g. n. sp. (Trichoptera, Limnephilidae). Acta Societatis Zoologicae Bohemoslovenicae, 16(1-2): 1-22.

BotosaneAnu, L. (1957a): Badania nad chruscikami (Trichoptera) Rumunii. Bulletin Entomologique de la Pologne, 26(25): 383-433.
BotosaAneanu, L. (1957b): Quelques Trichopteres nouveaux de Roumanie. Tijdschrift voor Entomologie, 100(2): 179-194.

BotosAnEANU, L. (1961): Materiaux pour server a la connaissance des Trichopteres d'Europe Orientale et Centrale. Folia Entomologica Hungarica, 14(2): 11-91.

Botosaneanu L. (1965): Neue Trichopterologische Fänge in Polen, Rumanien und Bulgarien. Latvijas Entomologs, 10: 53-60.

BotosAnEANU, L. (1967): Isogamus lineatus Klapálek (Trichoptera, Limnophilidae). Annalen des Naturhistorischen Museum in Wien, 70: 99-106.

BotosANEANU, L. (1973): Les Trichoptéres (Insecta: Trichoptera) de l'espace Carpato-Balkanique, fournisseurs de documents pour l'étude de l'evolution. Rivista di Idrobiologia (Perugia), 12(2-3): 119 152.

BotosaneANU, L. (1994): Two new species of $M e$ lampophylax Schmid 1955 from France (Trichoptera: Limnephilidae). Entomologische Zeitschrift, 104(18): 363-366.

BotosAnEANU, L. (1995): Additional documents to the knowledge of the Trichoptera of Romania, with data on European taxa from outside this country (Insecta: Trichoptera). Faunistische Abhandlungen Staatliches Museum für Tierkunde Dresden, 20(6): 57-88.

BotosaneAnu, L. \& SCHNEIDER, E.A. (1978): Die Köcherfliegen (Trichoptera) in den Sammlungen des Naturwissenschaftlichen Museum Sibiu. Museul Brukenthal. Studii si Comunicari, 22: $307-$ 326.

BotOSANEANU L. \& RIEDEL, W. (1965): Contribution a la connaissance de la variabilité géographique de Rhadicoleptus alpestris Kol. (Trichoptera, Limnephilidae). Bulletin de l'Académie Polonaise des Sciences, Cl. II. 8(9): 545-551.

BuChanan, A.V., Weiss, K.M. \& Fullerton, S.M. (2006): Dissecting complex disease: the quest for the Philosopher's Stone? International Journal of Epidemiology, 35: 562-571. doi: 10.1093/ije/dyl001

BuDD, G.E \& OLSSON, L. (2007) Editorial: a renaissance for evolutionary morphology. Acta Zoologica, 88: 1. doi: 10.1111/j.1463-6395.2007.00262.x

Butlin, R.K. \& TREganzA, T. (1998) Levels of genetic polymorphism: markers versus quantitative traits. Philosophical Transactions of the Royal Society B, 353: 187-198. doi: 10.1098/rstb.1998.0201 
BUTLIN, R.K. et al. (with 25 co-authors of The Marie Curie Speciation Network) (2012): What do we need to know about speciation? Trends in Ecology and Evolution, 27(1): 27-39. doi: $10.1016 /$ j.tree.2011.09.002

Charlesworth, B. (1996): The evolution of chromosomal sex determination and dosage compensation. Current Biology, 6(2): 149-162. doi: 10.1016/S0960-9822(02)00448-7

Chen, S., Krinsky, B.H. \& Long, M. (2013): New genes as drivers of phenotypic evolution. Nature Reviews Genetics, 14: 645-660. doi: $\underline{10.1038 / \mathrm{nrg} 3521}$

ChVOJKA, P. (1993): New data on the caddisfly fauna (Trichoptera, Insecta) of Slovakia from the East Carpathians. Biologia, Bratislava, 48(2): 217-221.

CivetTA, A. \& SingH, R.S. (1998): Sex-related genes, directional sexual selection, and speciation. Molecular Biology and Evolution, 15(7): 901-909.

COYNE, J.A. \& ORR, H.A. (2004): Speciation. Sinauer Associates, Sunderland, MA, pp. 545.

CResPI, B. \& NosiL, P. (2012): Conflictual speciation: species formation via genomic conflict. Trends in Ecology and Evolution, 28(1): 48-57. doi: $10.1016 / \mathrm{j}$. tree.2012.08.015

CRISCI, J.V. (2006): One-dimensional systematist: perils in a time of steady progress. Systematic Botany, 31(1): 217-221. doi: 10.1600/036364406775971859

CuÉnot, L. (1936): L'Espéce. G. Doin et Cie, Paris, pp. 310

Dahdul, W.M., Cui, H., Mabee, P.M., Mungall, C.J., OSumi-Sutherland, D., Walls, R.L. \& HAENDEL, M. A. (2014): Nose to tail, roots to shoots: spatial descriptors for phenotypic diversity in the Biological Spatial Ontology. Journal of Biomedical Semantics, 5(34): 1-13. doi: $\underline{10.1186 / 2041-1480-5-34}$

DARWIN, C. (1859): On the origin of species by means of natural selection, or the preservation of favoured races in the struggle for life. John Murray, London, pp. 502. doi: $10.5962 / \mathrm{bhl}$.title.82303

DARWIN, C. (1871): Descent of man, and selection in relation to sex. Appleton, New York, pp. 409.

DAVIES, P.C. W. (2013): Exposing cancer's deep evolutionary roots. Physics World, 26(7): 37-40.

DAVIES, P.C.W. \& LINEWEAVER, C.H. (2011): Cancer tumors as Metazoa 1.0: tapping genes of ancient ancestor. Physical Biology, 8(1): 015001. doi: $\underline{10.1088 / 1478-3975 / 8 / 1 / 015001}$
DAYRAT, B. (2005): Towards integrative taxonomy. Biological Journal of the Linnean Society, 85: 407415. doi: 10.1111/j.1095-8312.2005.00503.x

DeAns, A.R., Yoder, M.J. \& BALHOFF, J.P. (2012a): Time to change how we describe biodiversity. Trends in Ecology and Evolution 27(2): 78-84. doi: $\underline{10.1016 / j . t r e e .2011 .11 .007}$

Deans, A.R., Miko, I., Wipfler, B. \& Friedrich, F. (2012b): Evolutionary phenomics and the emerging enlightenment of arthropod systematics. Invertebrate Systematics, 26(2): 323-330. doi: $10.1071 / \mathrm{IS} 12063$

DEANS, A. R. et al. (with 72 co-authors) (2015) Finding our way through phenotypes. PLOS Biology, 13(1): e1002033. doi: $\underline{10.1371 / \text { journal.pbio. } 1002033}$

Dowling, D.K., FriberG, U. \& Lindell, J. (2008): Evolutionary implications of non-neutral mitochondrial genetic variation. Trends in Ecology and Evolution, 23(10): 546-554. doi: $10.1016 /$ j.tree.2008.05.011

DuboIs, A. (2010): Taxonomy in the century of extinction: taxonomic gap, taxonomic impediment, taxonomic urgency. Taprobanica, 2(1): 1-5. doi: 10.4038/tapro.v2i1.2702

DZIEDZIELEWICZ, J. (1911): Nowe gatunki owadów chróscikowatych, zebrane we wschodnich Karpatach. (Novae species Trichopterorum in Montibus Karpaticis orientalibus collectae). Sprawozdania Komisji Fizjografiznej Akademii Umiejetnosci $w$ Krakowie, 45: 45-47.

DZIEDZIELEWICZ, J. (1912): Nowe gatunki owadów chróscikowatych (Trichoptera), zebrane we wschodnich Karpatach w ciagu lata 1911. Sprawozdania Komisji Fizjograficznej Akademii Umiejetnosci w Krakowie, 46: 132-139.

DZIEDZIELEWICZ, J. \& KLAPÁleK, F. (1908a): Novae species Neuropteroideorum in Karpathibus Orientalibus anno 1907 collectae. Casopis Ceske Spolecnosti Entomologicke (Acta Societatis Entomologicae Bohemiae), 5(1): 21-24.

DZIEDZIELEWICZ, J. \& KLAPÁleK, F. (1908b): Nowe gatunki owadow siatkoskrzydlych zebrane w ciagu lata 1907 we wschodnich Karpatach (Nowae species Neuropteroidorum anno 1907 in Karpathibus Orientalibus collectae). Kosmos Lwow, 33: 250 256.

EBERHARD, W.G. (2010): Cryptic female choice. Encyclopedia of animal behaviour, 1: 430-434. doi: 10.1016/B978-0-08-045337-8.00186-8

EVENHUIS, N.L. (2007): Helping solve the "other" taxonomic impediment: completing the eight steps 
to total enlightenment and taxonomic Nirvana. Zootaxa, 1407: 3-12.

FAIRBAIRN, D.J. \& RoFF, D.A. (2006): The quantitative genetics of sexual dimorphism: assessing the importance of sex-linkage. Heredity, 97: 319-328. doi: $10.1038 /$ sj.hdy.6800895

FelsensteIn, J. (1981): Skepticism topwards Santa Rosalia, or why are there so few kinds of animals? Evolution, 35(1): 124-138. doi: $\underline{10.2307 / 2407946}$

FitZPATRICK, B.M., FordyCE, J.A. \& GAVRILETS, S. (2008): What, if anything, is sympatric speciation? Journal of Evolutionary Biology, 21(6): 14521459. doi: $\underline{10.1111 / \mathrm{j} .1420-9101.2008 .01611 . \mathrm{x}}$

FLEGR, J. (1996): Environmental selection, the missing term in Darwinism. Acta Societatis Zoologicae Bohemicae, 60: 95-96.

FLOWERS, R.W. (2007): Comments on „Helping solve the ,other" taxonomic impediment: completing the eight steps to total enlightenment and taxonomic Nirvana" by Evenhuis (2007). Zootaxa, 1494: 6768.

Franco, F.E., Prado, P.R.R., SENE, F.M., Costa, L.F. \& MANFRIN, M.H. (2006): Aedeagus morphology as a discriminant marker in two closely related cactophilic species of Drosophila (Diptera; Drosophilidae) in South America. Annals of the Brazilian Academy of Sciences, 78(2): 203-212.

FRIEDRICH, F. \& BEUTEL, R.G. (2008): Micro-computer tomography and a renaissance of insect morphology. Proceedings - Society of Photo-Optical Instrumentation Engineers 7078, Developments in X-Ray Tomography VI, $70781 \mathrm{U}$. doi: $10.1117 / 12.794057$

Galtier, N., NABholz, B., GlÉmin, S. \& HuRst, G.D.D. (2009): Mitochondrial DNA as a marker of molecular diversity: a reappraisal. Molecular Ecology, 18: 4541-4550. doi: 10.1111/j.1365-294X.2009.04380.x

Gibson, J.R., ChIPPINDAle, A.K. \& RICE, W.R. (2001): The $\mathrm{X}$ chromosome is a hot spot for sexually antagonistic fitness variation. Proceedings of the Royal Society of London B, 269: 499-505. doi: $\underline{10.1098 / \mathrm{rspb} .2001 .1863}$

Goldstein, D.B. \& Weale, M.E. (2001): Population genomics: Linkage disequilibrium holds the key. Current Biology, 11: 576-579. doi: 10.1016/S0960-9822(01)00348-7

GooD, J.M. et al. (with 10 co-authors) (2013): Comparative population genomics of the ejaculate in humans and the great apes. Molecular Biology and Evolution, 30(4): 964-976. doi: $10.1093 / \mathrm{molbev} / \mathrm{mst} 005$

HaERty, W., Jagadeeshan, S., Kulathinal, R.J., Wong, A., RAM, K.R., SiRot, L.K., LEVESQUE, L., Artieri, C.G., Wolfner, M.F., CivetTA, A. \& SINGH, R. S. (2007): Evolution in the fast lane: rapidly evolving sex-related genes in Drosophila. Genetics, 177: 1321-1335. doi: $10.1534 /$ genetics.107.078865

Hagen, H.A. (1961): Die Phryganiden Pictet's nach Typen Bearbeitet. Stettiner Entomologische Zeitung, 22: 113-117.

HAUSDORF, B. (2011): Progress toward a general species concept. Evolution, 65(4):923-931. doi: 10.1111/j.1558-5646.2011.01231.x

He, T. \& LAmonT, B.B. (2010): High microsatellite genetic diversity fails to predict greater population resistance to extreme drought. Conservation Genetics, 11: 1445-1451. doi: 10.1007/s10592-009-9971-4

Hendry, A.P., NosiL, P. \& RIESEBERG, L.H. (2007): The speed of ecological speciation. Functional Ecology 21(3): 455-464. doi: 10.1111/j.1365-2435.2007.01240.x

HEY, J. (2006): Recent advances in assessing gene flow between diverging populations and species. Current Opinion in Genetics \& Development, 16: 592-596. doi: 10.1016/j.gde.2006.10.005

Houle, D. (2010): Numbering the hairs on our heads: The shared challenge and promise of phenomics. Proceedings of the National Academy of Sciences, 107(suppl 1): 1793-1799. doi: 10.1073/pnas.0906195106

Houle, D., Govindaraju, D.R. \& OMHOlt, S. (2010): Phenomics: the next challenge. Nature Reviews Genetics, 11: 855-866. doi: 10.1038/nrg2897

Hotzy, C., Polak, M., RÖnN, J.L. \& ARnQvist, G. (2012): Phenotypic engineering unveils the function of genital morphology. Current Biology, 22: 2258-2261. doi: 10.1016/j.cub.2012.10.009

HubBELl, S.P. (1979): Tree dispersion, abundance, and diversity in a tropical dry forest. Science, 203: 1299-1309. doi: 10.1126/science.203.4387.1299

HuXLEY, J.S. (1940) The new systematics. Clarendon Press, Oxford, pp. 583.

JagadeEShan, S. \& Singh, R.S. (2006): A timesequence functional analysis of mating behaviour and genital coupling in Drosophila: role of cryptic female choice and male sex-drive in the evolution of male genitalia. Journal of Evolutionary Biology, 19(4): 1058-1070. doi: 10.1111/j.1420-9101.2006.01099.x 
JENNINGS, J.H. \& ETGES, W.J. (2010): Species hybrids in the laboratory but not in nature: a reanalysis of premating isolation between Drosophila arizonae and D. mojavensis. Evolution, 64: 587-598. doi: $10.1111 / \mathrm{j} .1558-5646.2009 .00834 . \mathrm{x}$

JOHNSON, N. (2011): A collaborative, integrated and electronic future for taxonomy. Invertebrate Systematics, 25: 471-475. doi: 10.1071/IS11052

Juenger, T., PÉreZ-PÉreZ, J.M., BernAl, S. \& MiCOL, J.L. (2005): Quantitative trait loci mapping of floral and leaf morphology traits in Arabidopsis thaliana: evidence for modular genetic architecture. Evolution \& Development, 7(3): 259-271. doi: 10.1111/j.1525-142X.2005.05028.x

KAMIMURA, Y. (2007): Twin intromittent organs of Drosophila for traumatic insemination. Biology Letters, 3: 401-404. doi: 10.1098/rsbl.2007.0192

KANESHIRO, K.Y. (2006): Dynamics of sexual selection in the Hawaiian Drosophilidae: a paradigm for evolutionary change. Proceedings of the Hawaiian Entomological Society, 38: 1-19.

KASIANOWICZ, J.J., BRANDIN, E., BRANTON, D. \& DEAMER, D.W. (1996): Characterization of individual polynucleotide molecules using a membrane channel. Proceedings of the National Academy of Sciences, 93: 13770-13773. doi: $10.1073 /$ pnas.93.24.13770

KIMURA, M. \& CROW, J. F. (1964): The number of alleles that can be maintained in a finite population. Genetics, 94: 725-738.

KIRKPATRICK, M. \& HALL, D.W. (2004): Sexual selection and sex linkage. Evolution, 58(4): 683-691. doi: 10.1111/j.0014-3820.2004.tb00401.x

KLAPÁLEK, F. (1901): O nových a málo známých druzích palaearktických Neuropteroid. Rozpravy České Akademie Cisare Frantiska Josefa pro Védy, Slovesnost a Uméni, Praze, (2) 10(21): 1-19.

KLAPÁLEK, F. (1907): Prispevek k znalosti zwireny chrostiku a jepic Vych. Karpat. (Additamentum ad Trichopterorum ac Ephemeridorum in Karpathibus faunae cognitionem). Casopis Ceské Spolecnosti Entomologické (Acta Societatis Entomologicae Bohemiae), 4: 24-36.

KolaczKowski, B., KeRn, A.D., Holloway, A.K. \& BEGUN, D. (2010): Genomic differentiation between temperate and tropical Australian populations of Drosophila melanogaster. Genetics, 187: 245260. doi: $10.1534 /$ genetics.110.123059

Kolenati F.A. (1848) Genera et species Trichopterorum. Pars Prior. Actis Regiae Bohemicae Societatis Scientiarum, 6: 1-108, 3 pl.
KolENATI, F.A. (1860): Einige neue Insecten-Arten vom Altvater. Wiener entomologischen Monatsschrift, 4: 381-394.

KopP, M. (2010): Speciation and the neutral theory of biodiversity: modes of speciation affect pattern of biodiversity in neutral communities. Bioessays, 32(7): 564-570. doi: 10.1002/bies.201000023

KRAAIJEVELD, K., KRAAIJEVELD-SMIT, F.J.L. \& MAAN, M.E. (2011): Sexual selection and speciation: the comparative evidence revisited. Biological Reviews, 86(2): 367-377. doi: $10.1111 /$ j.1469-185X.2010.00150.x

KuCINIC M., SZIVÁK, I., PAUls, S.U., BÁlint, M., DELIC, A. \& VucKOVIC, I. (2013): Chaetopteryx bucari sp. n., a new species from the Chaetopteryx rugulosa group from Croatia (Insecta, Trichoptera, Limnephilidae) with molecular, taxonomic and ecological notes on the group. ZooKeys, 320: 1-28. doi: $\underline{10.3897 / \text { zookeys. } 320.4565}$

Kulathinal, R.J. \& SinGH, R.S. (2004): The nature of genetic variation in sex and reproductive-related genes among sibling specie of Drosophila melanogaster complex. Genetica, 120: 245-252. doi: 10.1007/978-94-007-0965-2_20

LACKEY, A.C.R. \& Boughman, J.W. (2014): Female discrimination against heterospecific mates does not depend on mating habitat. Behavioral Ecology, 5: 1256-1267. doi: 10.1093/beheco/aru111

LEINONEN, T.L., MCCAIRNS, R.J.S., O’HARA, R.B. \& MERILA, J. (2013): Qst - Fst comparisions: evolutionary and ecological insights from genomic heterogeneity. Nature Reviews Genetics, 14: 179190. doi: $10.1038 / \operatorname{nrg} 3395$

LI, W.-H., YI, S. \& MAKOVA, K. (2002): Male-driven evolution. Current Opinion in Genetics and Development, 12: 650-656. doi: 10.1016/S0959-437X(02)00354-4

LINEWEAVER, C.H., DAVIES, P.C.W. \& VINCENT, M.D. (2014): Targeting cancer's weaknesses (not its strengths): Therapeutic strategies suggested by the atavistic model. Bioessays, 36(9): 827-835. doi: $10.1002 /$ bies. 201400070

LIU, Z.J. \& CORDES, J.F. (2004): DNA marker technologies and their applications in aquaculture genetics. Aquaculture, 238: 1-37. doi: $10.1016 /$ j. aquaculture.2004.05.027

LiUkART, G.L., ENGLAND, P.R., TALlMON, D., JoRDAN, S. \& TABERLET, P. (2003): The power and promise of population genomics: from genotyping to genome typing. Nature Reviews Genetics, 4: 981-994. doi: $10.1038 / \operatorname{nrg} 1226$ 
Lyon, B.E. \& MONTGOMERIE, R. (2012): Sexual selection is a form of social selection. Philosophical Transactions of the Royal Society B, 367: 22662273. doi: $10.1098 / \mathrm{rstb} .2012 .0012$

MAAN, M.E. \& SeEhausen, O. (2011): Ecology, sexual selection and speciation. Ecology Letters, 14: 591-602. doi: $10.1111 / \mathrm{j} .1461-0248.2011 .01606 . \mathrm{x}$

MAAN, M.E. et al. (with 9 co-authors) (2004): Intraspecific sexual selection on a speciation trait, male coloration, in the Lake Victoria cichlid Pundamilia nyererei. Proceedings of the Royal Sociaty of London B, 271: 2445-2452. doi: $10.1098 / \mathrm{rspb} .2004 .2911$

MACKAY, T.F.C. (2014): Epistasis and quantitative traits: using model organisms to study gene-gene interactions. Nature Reviews Genetics, 15: 22-33. doi: $10.1038 / \mathrm{nrg} 3627$

MaLiCKY, H. (1983): Atlas of European Trichoptera. Dr W. Junk, The Hauge, Boston, London, pp. 298.

MaLiCKY, H. (1990): Revision der Gattung Melampophylax Schmid, 1955 (Trichoptera, Limnephilidae). Nachrichtenblatt der Bayerischen Entomologen 39(1):1-12.

MALICKY, H. (1996): Das Problem der allopatrischen Arten bei Europäischen Köcherfliegen (Insecta: Trichoptera). Natura Croatica, 5: 11-23.

MALICKY, H. (2001): Notes on the taxonomy of Rhadicoleptus, Ptilocolepus and Pseudoneureclipsis. Braueria, 28: 19-20.

MaLICKY, H. (2004): Atlas of European Trichoptera. Second Edition. Springer, Dordrecht, The Netherlands, pp. 359.

MALICKY, H. 2005. Ein kommentiertes Verzeichnis der Köcherfliegen (Trichoptera) Europas und des Mediterrangebietes. Linzer biologische Beitrage, 37(1):533-596.

MaLiCKY, H. (2014): Comments on two recently published papers on Cheumatopsyche (Hydropsychidae) and Chaetopteryx (Limnephilidae). Braueria, 41: 51-53.

Malicky H., KrUSNiK, C., MoRetTi, G. \& NÓGRÁDI, S. (1986): Ein Beitrag zur Kenntnis der Chaetopteryx rugulosa Kolenati, 1848 - Gruppe (Trichoptera, Limnephilidae). Entomofauna, Zeitschrift für Entomologie, 7(1): 1-27.

MALICKY H. \& PAULS, S.U. (2012): Cross-breading of Chaetopteryx morettii and related species, with molecular and eidonomical results (Trichoptera,
Limnephilidae). Annales de Limnologie, 48: 13-19. doi: $10.1051 / \mathrm{limn} / 2011054$

Mank, J.E., Hultin-RosenberG, L., Webster, M.T. \& Ellengren, H. (2008): The unique genomic properties of sex-biased genes: insights from avian microarray data. BMC Genomics, 9: 148. doi: $\underline{10.1186 / 1471-2164-9-148}$

MANK, J.E. (2009): Sex chromosomes and the evolution of sexual dimorphism: lessons from the genome. The American Naturalist, 173(2): 141150. doi: $10.1086 / 595754$

MANK, J.E. (2012): Small but mighty: the evolutionary dynamics of $\mathrm{W}$ and $\mathrm{Y}$ sex chromosomes. Chromosome Research, 20: 21-33. doi: 10.1007/s10577-011-9251-2

MANK, J.E., NAM, K. \& ElLEGREN, H. (2010): Faster-Z evolution is predominantly due to genetic drift. Molecular Biology and Evolution, 27(3): 661-670. doi: $10.1093 / \mathrm{molbev} / \mathrm{msp} 282$

Mateus, C.S., Alves, M. J., Quintella, B. R. \& AlMEIDA, P.R. (2013): Three new cryptic species of the lamprey genus Lampetra Bonnaterre, 1788 (Petromyzontiformes: Petromyzontidae) from the Iberian Peninsula. Contributions to Zoology, 82 (1) $37-53$

MAYR, E. (1942): Systematics and the origin of species. Columbia University Press, New York, pp. 334.

MAYR, E. (1953): Methods and principles of Systematic Zoology. McGraw-Hill Book Company, New York, pp. 336.

MACARTHUR, R.H. (1972): Geographic ecology. Harper \& Row, New York, pp. 269.

MCKAY J.K., LATTA, R.G. (2002): Adaptive population divergence: markers, QTL and traits. Trends in Ecology and Evolution, 17: 285-291. doi: 10.1016/S0169-5347(02)02478-3

MCLACHLAN, R. (1874-1880): A monographic revision and synopsis of the Trichoptera of the European fauna. Reprinted 1968. E.W.Classey Ltd. Hampton, Middlesex, pp. 146. doi: $10.5962 /$ bhl.title.28556

MCLACHLAN, R. (1884) A monographic revision and synopsis of the Trichoptera of the European fauna. First Additional Supplement. Reprinted 1968. E.W.Classey Ltd. Hampton, Middlesex, pp. 76.

MCNEIL, C.L., BAIN, C.L. \& MACDONALD, S.J. (2011): Multiple Quantitative Trait Loci Influence the Shape of a Male-Specific Genital Structure in Drosophila melanogaster. Genes, Genoms, Genetics 1: 343-351. doi: 10.1534/g3.111.000661 
MEIER, R. (2008): DNA sequences in taxonomy. Opportunities and challenges. In. WHEELER, Q. (Ed.) The new taxonomy. The Systematic Association Special Volume Series, 76: 95-127. doi: 10.1201/9781420008562.ch7

Mey, W. \& Botosaneanu, L. (1985): Glazial-refugiale Subspeciation von Psilopteryx psorosa s.l. (Kolenati, 1860) in den Karpaten und angrenzenden Mittelgebirgen Zentraleuropas. Deutsche Entomoloische Zeitschrift, 32: 109-127.

Michel, A.P., SIM, S., POWELl, T.H.Q., TAYLOR, M.S., Nosil, P. \& FEDER, J.L. (2010): Widespread genomic divergence during sympatric speciation. Proceedings of National Academy of Sciences, 107(21): 9724-9729. doi: 10.1073/pnas.1000939107

MoretTI, G.P. (1991): Nouvelles espèces et sousespèces de Trichoptères italiens et exemples de variabilité des populations des Alpes meridionales et des Appenins. In. TOMASZEWSKI, C. (Ed.) Proceedings of the 6th International Symposium on Trichoptera, AMU Press, Poznan, p. 385-402.

Mosely, M.E. (1933): Synonyms in the order Trichoptera. Annals and Magazine of Natural History, Ser. 10, 12: 497-503. doi: $\underline{10.1080 / 00222933308673713}$

Mosely, M.E. (1934): New exotic Hydroptilidae. The Transactions of the Royal Entomological Society of London, 82: 137-163. doi: 10.1111/j.1365-2311.1934.tb00031.x

Mosely, M.E. (1937): Mexican Hydroptilidae (Trichoptera). The Transactions of the Royal Entomological Society of London, 86: 151-190. doi: 10.1111/j.1365-2311.1937.tb00242.x

Mosely, M.E. (1939): The Brasilian Hydroptilidae (Trichoptera). Novitates Zoologicae, 41: 217-239.

Moss, R., Piertney, S.B. \& PAlMER, S.C.F. (2003): The use and abuse of microsatellite DNA markers in conservation biology. Wildlife Biology, 9: 243250.

Murgoci, A. \& Botosaneanu, L. (1957): Genul Annitella Klap. in R.P.R. (Trichoptera). Analele Universitatii C. I. Parhon, Bucuresti, 13: 139-148.

NEI, M. (2005): Selectionism and neutralism in molecular evolution. Molecular Biology and Evolution, 22: 2318-2342. doi: 10.1093/molbev/msi242

Nelson, R.M., Pettersson, M.E. \& CARlborg, Ö. (2013): A century after Fisher: time for a new paradigm in quantitative genetics. Trends in Genetics, 29(12): 669-676. doi: $10.1016 /$ j.tig.2013.09.006
NIMMO, A.P. (1995): New species of Hydropsychidae and Limnephilidae (Insecta, Trichoptera) from the Far East of Russia, with description of a new genus of Limnephilidae (Limnephilini). Occasional Papers on Trichoptera Taxonomy, 1: 1-15.

Nosil, P. \& SCHLUTER, D. (2011): The genes underlying the process of speciation. Trends in Ecology and Evolution, 26(4): 160-167. doi: 10.1016/j.tree.2011.01.001

OHTA, T. (2003): Evolution by gene duplication revisited: differentiation of regulatory elements versus proteins. Genetica, 118: 209-216. doi: $\underline{10.1023 / \mathrm{A}: 1024130431575}$

OLÁH, J. (2010): New species and new records of Palearctic Trichopetara in the material of the Hungarian Natural History Museum. Annales Historico-Naturales Nationalis Hungarici, 102: 65-117.

OlÁH J. \& JOHANSON, K.A. (2008): Reasoning an appendicular and functional caddisfly genital terminology. Braueria, 35: 29-40.

OLÁH, J. \& JOHANSON, K.A. (2011): New Neotropical Hydroptilidae (Trichoptera). Annales historico-naturales Musei nationalis hungarici, 103: 117-255.

OLÁH J. \& FLINT, O.S. jr. (2012): Description of new species in the Leucotrichini tribe (Trichoptera: Hydroptilidae). Annales historico-naturales Musei nationalis hungarici, 104: 131-213.

OLÁH, J., KovÁCS, T. (2012): New species and records of autumnal Trichoptera from Albania. Folia Historico Naturalia Musei Matraensis, 36: 89-104.

OlÁH, J., KovÁcs, T., Sivec, I., SzIVÁK, I. \& UrbANIC, G. (2012): Seven new species in the Chaetopteryx rugulosa species group: applying the phylogenetic species concept and the sexual selection theory (Trichoptera, Limnephilidae). Folia Historico Naturalia Musei Matraensis, 36: 51-79.

OLÁH, J. \& ITO, T. (2013a): Synopsis of the Oxyethira flavicornis species group with new Japanese Oxyethira species (Trichoptera, Hydroptilidae). Opuscula Zoologica, Budapest, 44(1): 23-46.

OlÁH, J., IBRAHIMI, H. \& KovÁCS, T. (2013b): The Chaetopteroides genus (Trichoptera, Limnephilidae) revised by fine structure analysis of parameres. Folia Historico Naturalia Musei Matraensis, 37: 93-108.

Oláh, J., Andersen, T., Chvojka, P., Coppa, G., GraF, W., IBrahimi, H., LODOVICI, O., PreviŠIĆ, A. \& VAlle, M. (2013c): The Potamophylax nigricornis group (Trichoptera, Limnephilidae): 
resolution of phylogenetic species by fine structure analysis. Opuscula Zoologica, Budapest, 44(2): 167-200.

Oláh, J., Chvojka, P., Coppa, G., Graf, W., IbraHIMI, H., LOdOVICI, O., RuIZ Garcia, A., SÁINZBARIÁIN, M., VAlle, M. \& ZAMORA-MuÑOZ, C. (2014): The genus Allogamus Schmid, 1955 (Trichoptera, Limnephilidae): revised by sexual selection-driven adaptive, non-neutral traits of the phallic organ. Opuscula Zoologica, Budapest, 45(1): 33-82.

OlÁH, J. \& KovÁCS, T. (2014): New species and records of Balkan Trichoptera III. Folia Historico Naturalia Musei Matraensis, 38: 97-131.

ORR, H.A. (2001): The genetics of species differences. Trends in Ecology and Evolution, 16(7): 343-350. doi: 10.1016/S0169-5347(01)02167-X

Pauls, S.U., Graf, W., HaAs, P. Lumbsch, H. \& WARINGER, J. (2008): Grazers, shredders and filtering carnivores - The evolution of feeding ecology in Drusinae (Trichoptera: Limnephilidae: insights from a molecular phylogeny. Molecular Phylogenetics and Evolution, 46: 776-791. doi: 10.1016/j.ympev.2007.11.003

POPPER, H. \& SCHAFFNER, F. (1959): The renaissance of morphology. Gastroenterology, 37: 689-692.

PRESGRAVES, D.C. (2008): Sex chromosomes and speciation in Drosophila. Trends in Genetics, 24(7): 336-343. doi: 10.1016/j.tig.2008.04.007

ProuT, T. (1971): The relation between fitness components and population prediction in Drosophila. I. The estimation of fitness components. Genetics, 68 : 127-149.

PRUM, R.O. (2012): Aesthetic evolution by mate choice: Darwin's really dangerous idea. Philosophical Transactions of the Royal Society B, 367: 2253-2265. doi: 10.1098/rstb.2011.0285

DE QUEIROZ K. (2011): Branches in the lines of descent: Charles Darwin and the evolution of the species concept. Biological Journal of the Linnean Society, 103: 19-35. doi: 10.1111/j.1095-8312.2011.01634.x

QVARNSTRÖM, A. \& BAILEY, R.I. (2009): Speciation through evolution of sex-linked genes. Heredity, 102: 4-15. doi: 10.1038/hdy.2008.93

RACIECKA, M. (1934): Neue Diagnosen der von J. Dziedzielewicz beschriebenen Trichopterenformen. Konowia, 13: 231-245.

RAMME, W. (1930): Revisionen und Neubeschreibungen in der Gattung Pholidoptera Wesm. (Orth.,
Tettigon.). Mitteilungen aus dem Zoologischen Museum in Berlin, 16: 798-821.

REED, D.H. \& FRANKHAM, R. (2001): How closely correlated are molecular and quantitative measures of genetic variation? A meta-analysis. Evolution, 55: 1095-1103. doi: 10.1111/j.0014-3820.2001.tb00629.x

Resh M.C., Shrubovych, J., Bartel, D., SzUCSiCH, N.U., Timelthaler, G., Bu, Y., Walzl, M. \& PASS, G. (2014): Where taxonomy based on subtle morphological differences is perfectly mirrored by huge genetic distances: DNA barcoding in Protura (Hexapoda). PLOS One, 9(3): e90653. doi: 10.1371/journal.pone.0090653

Rivera, A.C, ANDrÉs, J.A., CóRdobA-Aguilar, A. \& UTZERI, C. (2004): Postmating sexual selection: allopatric evolution of sperm competition mechanisms and genital morphology in calopterygid damselflies (Insecta: Odonata). Evolution 58(2): 349-359. doi: 10.1554/03-244

RonN, J., KATVAla, M. \& ARnQuist, G. (2007): Coevolution between harmful male genitalia and female resistance in seed beetles. Proceedings of the National Academy of Sciences, 104: 1092110925. doi: 10.1073/pnas.0701170104

Rosindell, J., HubBell, S.P. \& ETIENNE, R.S.(2011): The unified neutral theory of biodiversity and biogeography at age ten. Trends in Ecology and Evolution, 26(7): 340-348. doi: $10.1016 /$ j.tree.2011.03.024

RöN, J.L., KATVAla, M. \& ARnQuist, G. (2011): Correlated evolution between male and female primary reproductive characters in seed beetles. Functional Ecology, 25: 634-640. doi: 10.1111/j.1365-2435.2010.01809.x

SackTon, T.B., Corbett-Detig, R.B., NAgaraju, J., VAISHNA, L., ARUNKUMAR, K.P. \& HARTL, D.L. (2014): Positive selection drives faster-Z evolution in silkmoths. Evolution 68(8): 2331-2342. doi: 10.1111/evo.12449

SÆTRE, G.P. \& SÆTHER S.A. (2010): Ecology and genetics of speciation in Ficedula flycatchers. Molecular Ecology, 19: 1091-1106. doi: 10.1111/j.1365-294X.2010.04568.X

SASABE, M, TAKAMI, Y. \& SOTA, T. (2007): The genetic basis of interspecific differences in genital morphology of closely related carabid beetles. Heredity, 98: 385-391. doi: 10.1038/sj.hdy.6800952

SChafer, M.A., RoutTu, J., VIEIRA, J., HOIKKALA, A., Ritchie, M.G. \& SCHLÖTTERER, C. (2011): Multiple quantitative trait loci influence intra-specific 
variation in genital morphology between phylogenetically distinct lines of Drosophila Montana. Journal of Evolutionary Biology, 24: 1879-1886. doi: 10.1111/j.1420-9101.2011.02316.x

SCHMID, F. (1952a): Contribution a l'étude des Trichopteres d'Espagne. Instituto de Estudios Pirenaicos Consejo Superior de Investigaciones Cientificas, Zaragoza, 1952: 627-695.

SCHMID, F. (1952b): Le groupe de Chaetopteryx (Limnophilidae, Trichoptery). Revue Suisse de Zoologie, 59(3): 99-171.

SCHMID, F. (1955): Contribution a l'étude des Limnophilidae (Trichoptera). Mitteilungen der Schweizerischen Entomologischen Gesellschaft, 28: 1245.

SCHMID, F. (1956): La Sous-Famille des Drusinae (Trichoptera, Limnephilidae). Mémoires Institut royal des Sciences naturelles de Belgique, deuxieme serie 55: $1-92+18$ plates.

SCHMID, F. (1998): The insects and arachnids of Canada. Part 7. Genera of the Trichoptera of Canada and adjoining or Adjacent United States. NRC Research Press, Ottawa, Ontario, Canada, pp. 319.

Scotland, R., Hughes, C., Bailey, D. \& Wortley, A. (2003): The Big Machine and the much-maligned taxonomist. Systematics and Biodiversity, 1(2): 139-143. doi: 10.1017/S1477200003001178

SEEHAUSEN, O. et al. (with 33 co-authors) (2014): Genomics and the origin of species. Nature Review Genetics, 15: 176-192. doi: 10.1038/nrg3644

Servedio, M.R., VAnDoorn, G.S., KoPP, M., Frame, A.M. \& NosIL, P. (2011): Magic traits in speciation: 'magic' but not rare. Trends in Ecology and Evolution, 26(8): 389-397. doi: $10.1016 / j . t r e e .2011 .04 .005$

SHAW, K.L. \& MulLEN, S P. (2011): Genes versus phenotypes in the study of speciation. Genetica, 139: 649-661. doi: 10.1007/s10709-011-9562-4

SinGH, R.S. \&KULATHINAL, R. . (2000): Sex gene pool evolution and speciation: a new paradigm. Genes and Genetic Systems, 75: 119-130. doi: $10.1266 /$ ggs. 75.119

Singh, R. \& JAGADEESHAN, S. (2012): Sex and speciation: Drosophila reproductive tract proteins twenty five years later. International Journal of Evolutionary Biology, 2012: 191495. doi: $10.1155 / 2012 / 191495$

SOTA, T. \& TANABE, T. (2010): Multiple speciation events in an arthropod with divergent evolution in sexual morphology. Proceedings of the Royal Society B, 277: 698-696. doi: 10.1098/rspb.2009.1822

Soto, I.M., CARREIRA, V.P., FANARA, J.J. \& HASSON, E. (2007): Evolution of male genitalia: environmental and genetic factors affect genital morphology in two Drosophila sibling species and their hybrids. BMC Evolutionary Biology, 7: 77. doi: $10.1186 / 1471-2148-7-77$

SOZZANI, R. \& BENFEY, P.N. (2011): High-throughput phenotyping of multicellular organisms: finding the link between genotype and phenotype. Genome Biology, 12(219): 1-7. doi: 10.1186/gb-2011-12-3-219

SPECTOR, R. (2010): The war on cancer a progress report for skeptics. Skeptical Inquirer, 34(1): 25-31.

STEIN, J.P.E.F. (1874): Beitrage zur Kenntniss der Phryganeiden des Altvaters und einiger anderer. Stettiner Entomologische Zeitung, 35: 244-253.

STRONG, D.R. Jr. (1980): Null hypotheses in ecology. Synthese, 43: 271-285. doi: 10.1007/BF00413928

Stuessy, T.F., MAYER, V. \& HÖRANDL, E. (2003): Deep morphology: toward a renaissance of morphology in plant systematics. Regnum Vegetabile, 141: $1-326$.

SWANSON, W.J. \& VACQUIER, V.D. (2002): The rapid evolution of reproductive proteins. Nature Review Genetics, 3: 137-144. doi: 10.1038/nrg/733

SZCZESNY, B. (1966): New and rare species of caddisflies (Trichoptera) in the fauna of Poland. Acta Hydrobiologica, 8(3-4): 341-346.

SZCZESNY, B. (1979): On the taxons of the genus Annitella Klapálek, 1907 (Trichoptera, Chaetopterygini) of the chomiacensis-lateroproducta group. Bulletin de l'academie Polonaise des Sciences, Cl.II, 27(4): 251-261.

SZCZESNY, B. (1980): Caddis-flies (Trichoptera) in the collection of the Institute of Systematic and EXperimental Zoology, Polish Academy of Science sin Cracow. Acta Zoologica Cracoviensia, 24(10): 449-486.

SZCZESNY, B. (1986): Caddisflies (Trichoptera) of running waters in the Polish North Carpathians. Acta Zoologica Cracoviensia, 29(21): 501-586.

SZCZESNY, B. (1987): A propos Psilopteryx proposa Kol. in the North Carpathians. Trichoptera Newsletter, 14: 25-26.

SZCZESNy, B. (1990): Potamophylax cingulatus Stephens - a polymorphic species? Trichoptera Newsletter, 17: 11-13. 
TAYLOR, J.S. \& RAES, J. (2004): Duplication and divergence: the evolution of new genes and old ideas. Annual Review of Genetics, 38: 615-643. doi: 10.1146/annurev.genet.38.072902.092831

TATARENKOV A. \& AVISE, J.C. (2007): Rapid concerted evolution in animal mitochondrial DNA. Proceedings of the Royal Society B, 274: 17951798. doi: $10.1098 / \mathrm{rspb} .2007 .0169$

Thomson, C.E., Gilbert, J.D.J. \& DE L. Brooke M. (2014): Cytochrome b divergence between avian sister species is linked to generation length and body mass. PLOS One 9(32): e85006. doi: 10.1371/journal.pone.0085006

Tobias, J.A., Seddon, N., Spottiswoode, C.N., PILGRIM, J.D., FISHPOOL, L.D.C. \& PILGRIM, J.D. (2010) Quantitative criteria for species delimitation. Ibis, 152: 724-746. doi: 10.1111/j.1474-919X.2010.01051.x

TOMKINS, J. \& BERGMAN, J. (2013): Incomplete lineage sorting and other "rogue" data fell the tree of life. Journal of Creation, 27(3): 84-92.

Turner, T.L., Bourne, E.C., Von WettBerg, E J., Hu, T.T. \& NuZHDIN, S.V. (2010): Population resequencing reveals local adaptation of Arabinopsis lyrata to serpentine soils. Nature Genetics, 42: 260-263. doi: $10.1038 / \mathrm{ng} .515$

VACQUIER, V.D. (1998): Evolution of gamete recognition proteins. Science, 281: 1995-1998. doi: $10.1126 /$ science. 281.5385 .1995

VAN DOORN, G.S., DICKMANN, U. \& WEISSING. F.J. (2004): Sympatric Speciation by Sexual Selection: A Critical Reevaluation. The American Naturalist, 163: 709-725. doi: $10.1086 / 383619$

Venkatesan, B.M. \& BASHIR, R.(2011): Nanopore sensors for nucleic acid analysis. Nature Nanotechnology, 6: 615-624. doi: $\underline{10.1038 / \text { nnano.2011.129 }}$

VShivkova, T.S., MORSE, J.C. \& RUTTER, D. (2007): Phylogeny of Limnephilidae and composition of the genus Limnephilus (Limnephilidae: Limnephilinae, Limnephilini). Proceedings of the XIIth International Symposium on Trichoptera. In. BUENOSoria, J., BARBA-Álvarez, R. \& ARMitage, B. (Eds.) The Caddis Press, Columbus, p. 309-319.

WALLENGREN H.D.J. (1891): Skandinaviens Neuroptera. Andra Afdelningen. Svenska VetenskapsAkademiens Handlingar, 24(10): 1-173.

WAringer, J., Botosaneanu, L. \& LeGuellec, G. (2011): The larva of Alpopsyche ucenorum (McLachlan 1876) (Trichoptera: Limnephilidae) with notes on its systematic position and ecology. Zootaxa, 2967: 44-50.

WEAVER, J.S. III (2002): A synonymy of the caddisfly genus Lepidotoma RAMBUR (Trichoptera: Lepidostomatidae), including a species list. Tijdschrift voor Entomologie, 145: 173-192.

WHEELER, Q.D. (2008): The new taxonomy. The Systematic Association Special Volume Series, 76: 1-37. doi: $10.1201 / 9781420008562$

Wiley, C., QvarnströM, A., Andersson, G., Borge, T. \& SÆTRE, G.P. (2009): Postzygotic isolation over multiple generations of hybrid descendents in a natural hybrid zone: how well do singlegeneration estimates reflect reproductive isolation? Evolution, 63(7): 1731-1739. doi: $10.1111 / \mathrm{j} .1558-5646.2009 .00674 . \mathrm{x}$

WILL, K.W., MiSHLER, B.D. \& WHEELER, Q.D. (2005): The peril of DNA barcoding and the need for integrative taxonomy. Systematic Biology, 54(5): 844-851. doi: 10.1080/10635150500354878

WILSON, E.O. (2003): The encyclopedia of life. Trends in Ecology and Evolution, 18(2): 77-80. doi: 10.1016/S0169-5347(02)00040-X

WinkLeR, K., RocQue, D.A., BRAILE, T.M. \& PRUETT, C.L. (2007): Vainly beating the air: species-concept debates need not impede progress in science or conservation. Ornithological Monographs, 63: 3044. doi: $10.2307 / 40166896$

Wolf, J.B.W., BAYER, T., HAubOld, B., Schilhabel, M., Rosenstiel, P. \& TAUTZ, D. (2010): Nucleotide divergence vs. gene expression differenttiation: comparative transcriptome sequencing in natural isolates from the carrion crow and its hybrid zone with the hooded crow. Molecular Ecology, 19(Suppl. 1): 162-175. doi: 10.1111/j.1365-294X.2009.04471.X

WyCKOFF, G.J., WANG, W. \& WU, C.I. (2000): Rapid evolution of male reproductive genes in the descent of man. Nature, 403: 304-309. doi: $10.1038 / 35002070$

ZEH, J.A. \& ZEH, D.W. (2003): Toward a new sexual selection paradigm: polyandry, conflict and incompatibility. Ethology, 109: 929-950. doi: 10.1046/j.1439-0310.2003.00945.x

ZHANG D.X. \& HEWITT, G.M. (2003): Nuclear DNA analysis in genetic studies of populations: practice, problems and prospects. Molecular Ecology, 12: 563-584. doi: 10.1046/j.1365-294X.2003.01773.X

Zhang Q., Zhang, F., Chen, X., Wang, Y., Wang, W., LIN, A.A., CAVAlli-SforZA, L.L., JIN, L., 
HuO, R., SHA, J.H., LI, Z. \& Su, B. (2007): Rapid evolution, genetic variations, and functional association of the human spermatogenesis-related gene NYD-SP12. Journal of Molecular Evolution, 65: $154-161$.

doi: $\underline{10.1007 / \mathrm{s} 00239-006-0127-6}$

YeH, S.D. et al. (with 14 co-authors) 2012. Functional evidence that a recently evolved Drosophila sperm- specific gene boosts sperm competition. Proceedings of the National Academy of Sciences, 109: 2043-2048. doi: 10.1007/s00239-006-0127-6

Yeates, D.K., Seago, A., Nelson, L., Cameron, S.L., Josheph, L. \& TRUEMAN, J.W.H. (2011): Integrative taxonomy, or iterative taxonomy? Systematic Entomology, 36: 209-217. doi: $\underline{10.1111 / j .1365-3113.2010 .00558 . x}$ 